

\section{DISCLAIMER}

This report was prepared as an account of work sponsored by an agency of the United States Government. Neither the United States Government nor any agency Thereof, nor any of their employees, makes any warranty, express or implied, or assumes any legal liability or responsibility for the accuracy, completeness, or usefulness of any information, apparatus, product, or process disclosed, or represents that its use would not infringe privately owned rights. Reference herein to any specific commercial product, process, or service by trade name, trademark, manufacturer, or otherwise does not necessarily constitute or imply its endorsement, recommendation, or favoring by the United States Government or any agency thereof. The views and opinions of authors expressed herein do not necessarily state or reflect those of the United States Government or any agency thereof. 


\section{DISCLAIMER}

Portions of this document may be illegible in electronic image products. Images are produced from the best available original document. 
Printed in the United States of America Available from

National Technical Information Service

U. S. Department of Commerce 5285 Port Royal Road

Springfield, Virginia 22161

Price: Printed Copy $\$ 7.75$; Microfiche $\$ 2.25$

\section{ANCR-1296, QUARTERLY TECHNICAL REPORT ON WATER REACTOR SAFETY PROGRAMS SPONSORED BY THE NUCLEAR REGULATORY COMMISSION'S DIVISION OF REACTOR SAFETY RESEARCH, JULY-SEPTEMBER 1975}
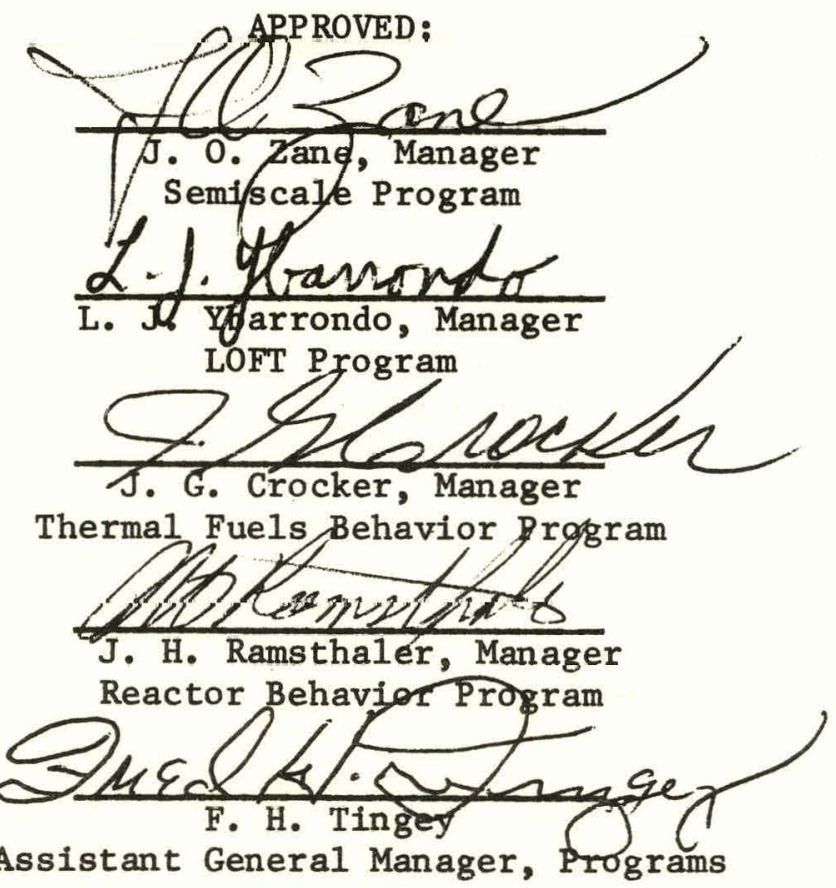

\section{NOTICE}

This report was prepared as an account of work sponsored by the United States Government. Neither the United States nor the Energy Research and Development Administration, nor the Nuclear Regulatory Commission, nor any of their employees, nor any of their contractors, subcontractors, or their employees, makes any warranty, express or implied, or assumes any legal liability or responsibility for the accuracy, completeness or usefulness of any information, apparatus, product or process disclosed, or represents that its use would not infringe privately owned rights. 


\title{
QUARTERLY TECHNICAL REPORT ON WATER REACTOR. SAFETY PROGRAMS SPONSORED BY THE NUCLEAR REGULATORY COMMISSION'S DIVISION OF REACTOR SAFETY RËSEARCH, JULY-SEPTEMBER 1975
}

\author{
AEROJET NUCLEAR COMPANY \\ IDAHO NATIONAL ENGINEERING LABORATORY \\ IDAHO FALLS, IDAHO 83401
}

Approved by Dr. F. H. Tingey

Date Published - February 1976

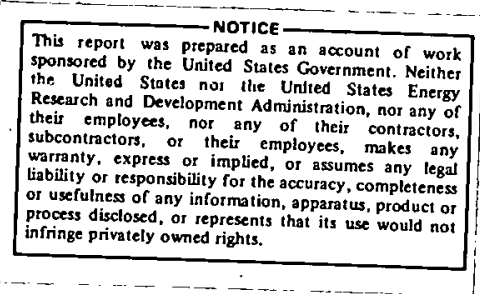

PREPARED FOR THE

U.S. NUCLEAR REGULATORY COMMISSION AND

ENERGY RESEARCH AND DEVELOPMENT ADMINISTRATION

IDAHO OPERATIONS OFFICE

UNDER CONTRACT NO. E(10-1)-1375 


\begin{abstract}
Light water reactor safety activities performed during July through September 1975 are summarized. The isothermal blowdown test series of the Semiscale Mod-1 test program has provided data for evaluation of break flow phenomena and analyses of piping flow regimes and pump performance. In the LOFT Program, measurement unccrtaintics were evaluated. The Thermal Fuels Behavior Program completed two power-cooling-mismatch tests on PWR-type fuel rods to investigate critical heat flux characteristics. Model development and verification efforts of the Reactor Behavior Program included development of the SPLEN1 computer code, subroutines for the FRAP-T code, verification of RELAP4, and results of the Halden Recycle Plutonium Experiment.
\end{abstract}




\section{PREFACE}

Aerojet Nuclear Company (ANC) performs technical activities in the water reactor safety programs at the Idaho National Engineering Laboratory (INEL) under the sponsorship of the U.S. Nuclear Regulatory Commission's Division of Reactor Safety Research. The current water reactor safety activities of ANC are accomplished in four programs: The Semiscale Program, the Loss-of-Fluid Test (LOFT) Program, the Thermal Fuels Behavior Program, and the Reactor Behavior Program.

The Semiscale Program consists of a continuing series of small-scale nonnuclear thermal-hydraulic experiments having as their primary purpose the generation of experimental data that can be applied to the development and verification of analytical models describing loss-of-coolant accident (LOCA) phenomena in water-cooled nuclear power plants. Emphasis is placed on acquiring system effects data from integral tests that characterize the most significant thermal-hydraulic phenomena likely to occur in the primary coolant system of a nuclear plant during the depressurization (blowdown) and emergency cooling phase of a LOCA. The current program of experiments employs a recently assembled test system, Semiscale Mod-1, which has one intact (operating) loop with active components and a broken (blowdown) loop with passive components. These experiments include isothermal blowdown tests (no core heating) and blowdown heat transfer and core reflood tests using an electrically heated 40-rod core.

The LOFT Program is a nuclear test program for providing test data to support: (a) assessment and improvement of the analytical methods utilized for predicting the behavior of pressurized water reactors (PWR) under LOCA conditions; (b) evaluation of the performance of PWR engineered safety features (ESF), particularly the emergency core cooling system (ECCS); and (c) assessment of the quantitative margins of safety inherent in the performance of these safety features. The test program utilizes the LOFT facility, an extensively instrumented 55-MW pressurized water reactor facility designed for the conduct of loss-of-coolant experiments (LOCE). The LOFT facility is in the final stages of construction. The test programs, planned to start in early 1976, include a series of nonnuclear (without nuclear heat) LOCEs followed by a series of low-power nuclear LOCEs and then a scrics of high-powcr nuclear LOCEs.

The Thermal Fuels Behavior Program is an integrated experimental and analytical program designed to provide information on the behavior of reactor fuels under normal, off-normal, and accident conditions. The experimental portion of the program is concentrated on testing of single fuel rods and fuel rod clusters under power-cooling-mismatch (PCM), loss-of-coolant, and reactivity initiated accident conditions. These tests provide in-pile experimental data for the evaluation and verification of analytical models that are used to predict fuel behavior under reactor conditions spanning normal operation through severe hypothetical accidents. Data from the Program provide a basis for improvement of the models. 
The Reactor Behavior Program encompasses the analytical aspects of predicting the response of nuclear power reactors under normal, abnormal, and accident conditions. Program areas include thermal hydraulic (loss of coolant accident analysis) code development, fuel code development, code verification, and code optimization. The program produces computer codes for predicting the behavior of reactor systems and fuel, establishes data requirements for and performs verification analysis of analytical safety assessment codes; and maintains and upgrades computer codes to meet changing analytical and verification requirements.

A more detailed description of the four programs is presented in quarterly technical report for January through March 1975, ANCR-1254. Copies of all Quarterly Reports issued by ANC are available from the Technical Information Center, Energy Research and Development Administration, Oak Ridge, Tennessee. 


\section{SUMMARY}

Water reactor safety program activities performed by Aerojet Nuclear Company during July through September 1975 are reported for the Semiscale Program, the LOFT Program, the Thermal Fuels Behavior Program, and the Reactor Behavior Program.

For the Semiscale Program, results from the isothermal blowdown test series of the Semiscale Mod-1 Test Program are discussed for three topical areas.

(1) An evaluation of break flow phenomena and the methods that best predicted them for the isothermal test series is presented. Knowledge of core fluid properties during blowdown of the Semiscale Mod-1 system is important to blowdown analysis in that high core flow rates and low fluid qualities result in more energy removal from the core. Core fluid properties are influenced by components within the system, principally the pump and the breaks, and accurate prediction of core phenomena can result only from properly accounting for the various influences imposed on the core from the components in the system. Analysis has shown that use of a critical break flow model in the RELAP4 code which correctly represents the physical phenomena occurring at the breaks is important in analytically determining the core fluid behavior. Of the present break flow options in RELAP4, the Henry break flow model (used for subcooled blowdown) followed by a sonic choking model (used for saturated blowdown) provides best results.

(2) The piping flow regimes that occurred during the isothermal test series are analyzed. Phase separation of the blowdown fluid was shown to occur in the Semiscale Mod-l piping. Comparison of the ealculated fluw regines will dald frum hurizunlal and vertical densitometers indicated that the calculation method used has reasonable utility in applications to a transient thermal-hydraulic process. Calculated flow regimes and flow regime transitions were found to be sensitive to broken loop break characteristics and locally sensitive to intact loop steam generator heal transfer. 
(3) An evaluation of intact loop pump performance is presented. Analysis of Semiscale Mod-1 isothermal test data showed that both the intact loop and vessel flow rates are influenced by the pump during the initial portion of the blowdown. As the pump inlet void fraction increases during blowdown, the pump head progressively degrades and the pump has: less effect on these flow rates. Pump performance has very little effect on fluid flow in the broken loop. The pump is affected by the flows that occur during the subcooled portion of the blowdown. The large break flow rates during the subcooled decompression cause an acceler-ation of flow at the intact loop pump inlet which tends to rapidly decrease pump differential pressure. When the break flows choke, the flow through the pump decelerates causing the pump head to partially recover. Analysis of the pump performance data has provided a new two-phase head degradation multiplier along with a recommended change in the single-phase homologous curvc. Use of these factors in a check calculation indicates that the pump differential pressure is more accurately calculated than with use of previous two-phase head degradation multipliers and homologous single-phase head curves.

An evaluation has been completed of anticipated performance uncertainty for each experimental measurement to be made in the LOFT Program. The LOFT Experimental Measurements Uncertainty Analysis is based on analysis of component test results, calculations, and equipment specifications. Results of the measurement uncertainty analysis are reported. Also reported is the experimental determination of LOFT primary coolant system hydraulic parameters relative to two system variables: (a) LOFT emergency core coolant (ECC) injection delay and (b) LOFT steam generator flow resistance. Minimum ECC injection delay (minimum cold wall delay) was determined (1.4 seconds) and will be compared with a hot wall delay effect which will be subsequently determined. Methods for predicting steam generator orifice size have been revised based on measured versus calculated flow resistances.

In the Thermal Fucls Behavior Program, results are reported for (wo powelcooling-mismatch tests performed on PWR-type fuel rods in the Power Burst Facility reactor.

(1) Metallurgical test results are presented for the second experiment (Test 8-1 RS) in the PCM-20 Test Series, which investigates preand post-critical heat flux (CHF) effects on a single 3-foot long PWR-type fuel rod: Peak fuel rod power required to achleve CHF was $15.9 \mathrm{~kW} / \mathrm{ft}$ for the first power cycle and 17.9 to $19.9 \mathrm{~kW} / \mathrm{ft}$ for the remaining three power cycles. Quasi-equilibrium film 
boiling occurred during the last two power cycles. The experiment coolant mass flux, inlet temperature, and pressure were $1 \times 10^{6} \mathrm{lb} / \mathrm{hr}^{-\mathrm{ft}^{2}}, 622^{\circ} \mathrm{F}$, and $2,080 \mathrm{psig}$, respectively. Location of the onset of CHF, as measured with the four cladding surface thermocouples, was between 25 and 29 inches above the bottom of the fuel stack. Time of CHF occurrence was reliably indicated by the fuel rod internal pressure measurement, the fuel centerline temperature measurement, and the cladding surface temperature measurements. These data correlated well with axial elongation data obtained with a linear variable differential transducer. 'Metallurical examination indicated the $\mathrm{UO}_{2}$ fuel underwent considerable restructuring in the region that experienced CHF. Restructuring consisted of grain growth which increased with increasing temperature toward the center of the fuel rod. The maximum measured fuel centerline temperature of $4,155^{\circ} \mathrm{F}$ occurred above the hottest region of the fuel rod. Cladding collapse and extensive cladding oxidation occurred over an 11-inch-long region of the fuel rod from 20 to 31 inches above the bottom of the fuel rod. Oxygen-stabilized alpha zirconium occurred both at the outside and inside of the cladding: source of the oxygen for the inside surface was most likely the $\mathrm{UO}_{2}$. However, small cracks occurred in the cladding in the heavily oxidized region and coolant may have entered the rod through these cracks. The fuel rod cladding surface thermocouple measurements were low by as much as $700^{\circ} \mathrm{F}$. Examination results clearly show that the method used to attach cladding surface thermocouples to the cladding for this test was inadequate for reliable temperature measurements.

(2) Preliminary test results of the third test in the PCM-20 Test Series are given. The test (PCM-20 CHF Scoping Test) was conducted to determine characteristic CHF occurrence parameters and was similar to the cyclic testing of a PWR type fuel rod as described above for the PCM-20 Test 8-1RS. Test results are tabulated.

In the Reactor Behavior Program, code development efforts are reported in the areas of loss-of-coolant accident (LOCA) analy sis and fuel rod model development.

(1) The SPLEN1 code was applied to two recirculating flow problems, one for a plane expansion junction and one for a plane tee junction. Analysis and experimental data show SPLEN1 can predict pressure drop and attendant form losses in a junction, wall shear stresses and friction losses, and trends in heat 
transfer rates. For the tee calculation, a recirculation zone occurs downstream from the junction and higher fluid velocities result from side branch flow into the junction.

(2) In fuel rod model development associated with FRAP-T code, a subroutine model (BALLOON) has been prepared to calculate the large deformation ballooning of reactor fuel cladding during rapid thermal transients. The model has been developed from first principles using membrane shell theory and compares favorably with applicable data. Parametric studies are presented to show both the numerical stability of the model and an inadequacy of available data with which to compare the model.

Reactor Behavior Program code verification efforts are discussed in fuel behavior and LUC'A analysis verificảtion reports.

(1) A comparison of calculated values with expenmental results is presented for a fuel rod cladding axial growth model, which was developed as part of the MATPRO subroutine to the FRAP-T code. The model calculates the fractional change in length of zircaloy tubes due solely to irradiation-induced growth and considers effects of fast neutron fluence, tubing texture, cladding temperature, and cold work. The comparison with data from cold worked tubes was not made because the extent of cold work was not known; dependence of cold worked tube growth on the square root of fluence was tested and is discussed.

(2) A summary description is presented of the Halden Recycle Plutonium Experiment and of data obtained during the steady state irradiation of an instrumented fuel assembly in the Halden Heavy Boiling Water Reactor. The experiment was conducted in conjunction with the NRC participation in the Organization for Economic Co-operation and Development (OECD) Halden Reactor Project. Fuel rod elongation (axial deformation), fuel centerline temperature, and fuel rod internal pressure data are presented as a function of power and time for rods with various initial pellet-to-cladding gap sizes and fuel densities. These data will be lised to facilitate devclopment and verification of analy tical models depicting fuel behavior.

(3) A condensation is presented of the ANC. model verification of the FRAP-S computer code. Results of data comparisons for many fuel behavior variables are provided. The reader is referred to a published reference for full details of the effort. 
LOCA analysis verification is discussed in two reports:

(1) A 12-node thermal hydraulic model of a PWR has been developed to permit investigation of several statistical approaches to a RELAP4-BE code uncertainty analysis. The statistical method selected through use of this simplified model will be later used for analysis with a more detailed PWR model and a complete set of the most influential LOCA parameters. This minimum node model has been shown to represent the best compromise between accuracy and computer running time.

(2) Data comparisons are described that are used to assess the capability of RELAP4 in predicting thermal-hydraulic response of the Semiscale Mod-1 simulated reactor core. Conclusions and recommendations of the study are presented which indicate that fuel rod cladding temperatures can be predicted with reasonable accuracy. 


\section{CONTENTS}

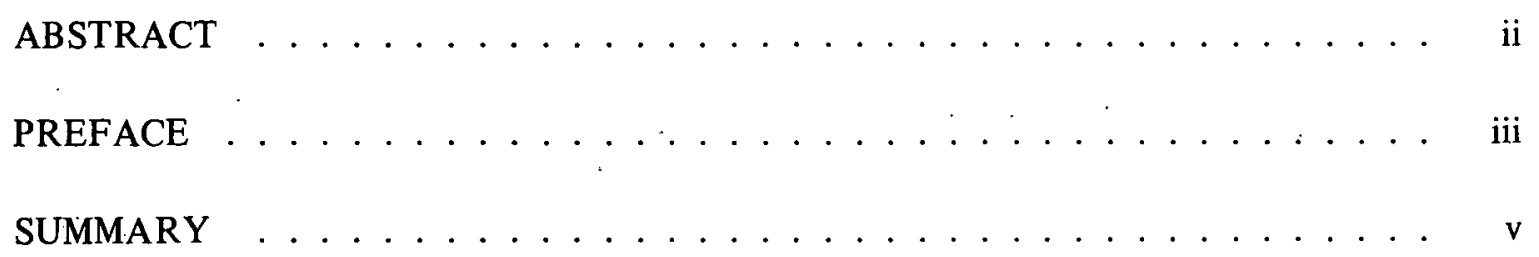

I. SEMISCALE PROGRAM $\ldots \ldots \ldots \ldots \ldots$

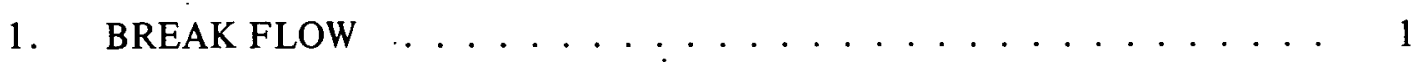

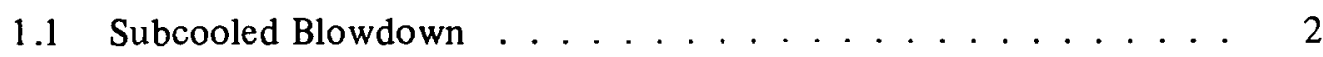

1.2 Saturated Blowdown $\ldots \ldots \ldots 6$

1.3 Break Flow Summary . . . . . . . . . . . 18

2. PIPING FLOW REGIMES IN THE SEMISCALE GEOMETRY _ . . . . 19

3. INTACT LOOP PUMP PERFORMANCE . . . . . . . . . . . . 25

3.1 Applicability of Steady State Single-Phase Test

Data to Describe Mod-1 Pump Performance . . . . . . . . . . 26

3.2 Applicability of the Steady State Two-Phase Tests to Describe Mod-1 Pump Performance . . . . . . . . . . . . . . 27

3.3 Applicability of Steady State Single- and Two-Phase Tests and Transient 1-1/2-Loop Tests for Analytically

Describing Mod-1 Pump Performance . . . . . . . . . . . 30

3.4 Development of a New Head Degradation Multiplier for Use in the RELAP4 Mndel . . . . . . . . . . . . . . . 32

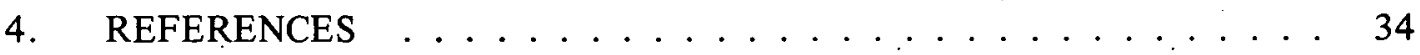

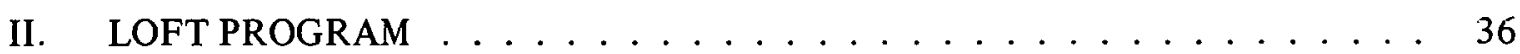

1. LOFT EXPERIMENTAL MEASUREMENTS UNCERTAINTY . . . . . .

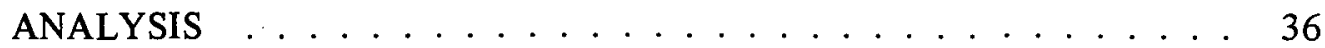

1.1 General Description ol" LOF"I' Lxpermental Measurement Systcm . . 36 1.2 Analysis Results . . . . . . . . . . . . . . 38

2. EXPERIMENTAL DETERMINATION OF LOFT PRIMARY COOLANT SYSTEM HYDRAULIC FARAMETERS . . . . . . . . . . . . . 58 
2.1 LOFT ECC Injection Delay . . . . . . . . . . . . 59

2.2 Steam Generator Flow Resistance . . . . . . . . . . . . . 62

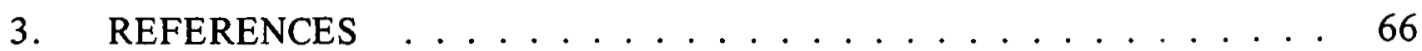

III. THERMAL FUELS BEHAVIOR PROGRAM . . . . . . . . . . . 67

1. PCM-20 TEST 8-1 RS, METALLURGICAL TEST RESULTS . . . . . . 67

1.1 Introduction .................... 67

1.2 Visual Examination ............... 67

1.3 Destructive Examination . . . . . . . . . . . 68

1.4 Summary of PIE Results . . . . . . . . . . . 86

2. PCM-20 CHF SCOPING TEST, PRELIMINARY TEST RESULTS _ . . . 87

2.1 Introduction . . . . . . . . . . . . . . 87

2.2 Test Configuration and Instrumentation . . . . . . . . 88

2.3 Power Calibration ................. 89

2.4 CHF Sçoping Tests . . . . . . . . . . . . . . 91

2.5 Posttest Inspection . . . . . . . . . . . . . . . 93

2.6. Conclusions ....................... 93

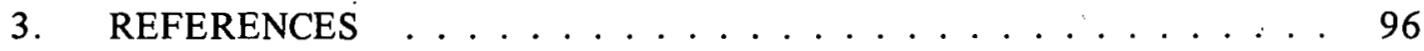

IV. REACTOR BEHAVIOR PROGRAM $\ldots \ldots \ldots \ldots \ldots$

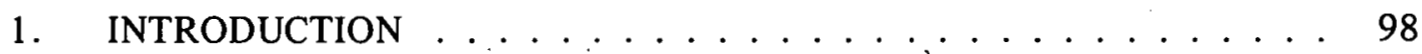

2. LOCA ANALYSIS DEVELOPMENT . . . . . . . . . . . . . . . . . 99

3. FUEL ROD MODEL DEVELOPMENT . . . . . . . . . . . . . 109

3.1 BALLOON: A Model to Calculate the Transient Ballooning of Reactor Fuel Cladding . . . . . . . . . . . . . . . 109

3.2 BALLOON Model Comparison Results . . . . . . . . . . . . 124

3.3 BALLOON Model Development Conclusions . . . . . . . . . 133

4. TULL DEIIAVIOR VERITICATION . . . . . . . . . . . . 134

4.1 Clàdding Axiàl Growth . . . . . . . . . . . . . . 134

4.2 Summary of Halden Recycle Plutonium Experiment . . . . . . 145

4.3 FRAP-S1 Model Verification . . . . . . . . . . . 178 


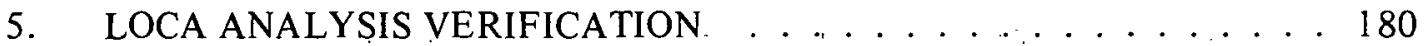

5.1 PWR Uncertainty Analysis Methods Study - 12-Node

Plant Model . . . . . . . . . . . . . . . . 180

5.2 Comparison of RELAP4 Predictive Capability with Semiscale MOD-1

Core Test Data . . . . . . . . . . . . . . 183

6. REFERENCES ...................... 186

\section{FIGURES}

I-1. Subcooled blowdown pressure at vessel and pump sides of break - Test

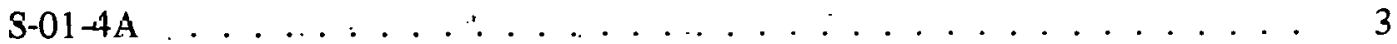

I-2. Subcooled blowdown tlow rate at vessel side of break - Tèst S-Ül-4A . . . . 4

I-3. Subcooled blowdown flow rate at pump side of break - Test S-01-4A . . . 4

I-4. Subcooled blowdown pressure - RELAP4 calculations for vessel side of

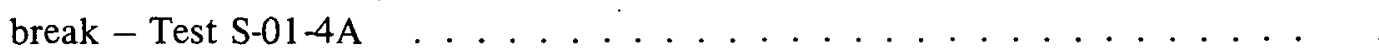

I-5. Subcooled blowdown flow rate - RELAP4 calculations for vessel side of break - Test S-01-4A . . . . . . . . . . . . . 7

I-6. Subcooled blowdown core flow rate - RELAP4 calculations for Test $\mathrm{S}-01-4 \mathrm{~A} \ldots \ldots \ldots \ldots . \ldots \ldots$

I-7. Saturated blowdown pressure at vessel side of break - Test S-01-4A . . . : 9

I-8. Saturated blowdown flow rate at vessel side of break - Test S-01-4A . . . . 9

I-9. Comparison of experimental and calculated saturated blowdown pressures at vessel side of break - Test $\mathrm{S}-01-4 \mathrm{~A} \ldots \ldots . \ldots 11$

I-10. Comparison of experimental and calculated saturated blowdown flow rates at vessel side of break - Test S-01-4A

I-1 1. Flow rate at vessel side of break - comparison of calculations with and without slip (Test S-01-1B) . . . . . . . . . . . . . 13

I-1 2. Flow rate at vessel side of break - comparison of results of $100 \%$ break (Test S-01-1 B) and of 200\% break (Test S-01-4A) . . . . . . . . 15 
I-13. Flow rate at entrance to core - Test S-01-2, S-01-3, S-01-4, and

$\mathrm{S}-01-5 \ldots \ldots \ldots \ldots \ldots$

I-14. Flow rate at entrance to core - comparison of RELAP4 results with

Test $\mathrm{S}-01-4 \mathrm{~A}$ data ......................... 17

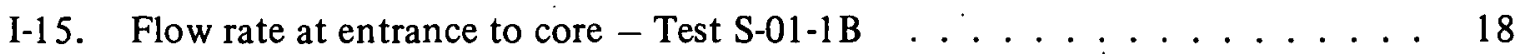

I-16. Flow regimes in the intact loop hot leg vessel outlet . . . . . . . . . . 20

I-17. Flow regimes shown by the vertical and horizontal density measurements at the in tact loop hot leg vessel outlet - Test $\mathrm{S}-01-4 \mathrm{~A} \ldots \ldots 21$

I-18. Flow regimes shown by the vertical and horizontal density measurements at the in tact loop hot leg vessel outlet - Test S-01-1B . . . . . . . . 21

I-19. Flow regimes in the intact loop cold leg vessel inlet $\ldots \ldots \ldots$

I-20. Flow regimes shown by the vertical and horizontal density measurements at the intact loop cold leg vessel inlet - Test S-01-4A . . . . . . . . . . 24

I-21. Flow regimes shown by the vertical and horizontal density measurements at the in tact loop cold leg vessel inlet - Test S-01-1 B . . . . . . . . . . 24

I-22. Semiscale Mod-1 pump characteristic curve with initial operating point for

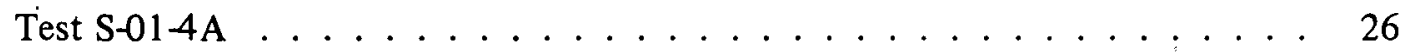

.I-23. Normalized head verus flow for various inlet void fractions from 0 to 10 seconds - Test $\mathrm{s}-01-3 \ldots \ldots \ldots 28$

I-24. Normalized head versus flow for various inlet void fractions from 10 to 20 seconds - Test $\mathrm{s}-01-3 \ldots \ldots \ldots \ldots . \ldots . \ldots . \ldots 29$

I-25. Normalized head versus flow for various inlet void fractions from 20 to 30 seconds - Test S-01-3 . . . . . . . . . . . . 29

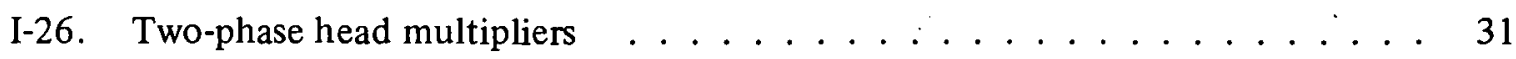

I-27. Test S-01-4A pump differential pressure calculated using RELAP4 pump model $\ldots \ldots \ldots \ldots \ldots \ldots \ldots \ldots$

I-28. Three two-phase multipliers for the Semiscale Mod-1 pump . . . . . . . 33

I-29. Pump differential pressure calculated using various multipliers . . . . . . 34

II-I. Block diagram of LUFT Experimental Measurement System . . . . . . . 37 
II-2. Cold wall delay experiment result - accumulator A to cold leg piping configuration ......................... 60

II-3. Calculated minimu cold wall delay . . . . . . . . . . . . . 63

II-4. Schematic of the LOFT steam generator piping and high-resistance orifice

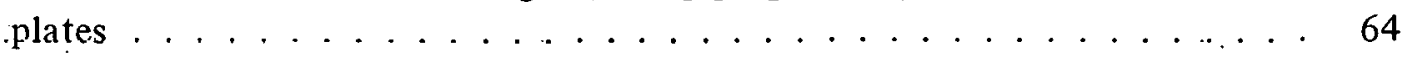

III-1. Stress-relieved structure in zircaloy cladding at 8-1/4 inches from bottom of fuel rod (PCM-20 Test 8-1 RS) . . . . . . . . . . . . . . 69

II-2. Recrystallized structure in zircaloy cladding, 31-7/8 inches from bottom of fuel rod (PCM-20 Test 8-1 RS) . . . . . . . . . . . . . . .

II-3. A longitudinal section of fuel rod at about 23 inches from bottom of rod showing prior $\pm \mathrm{Zr}$, oxygen stabilized $\alpha-\mathrm{Zr}, \alpha \mathrm{Zr}$.incursions, and $\mathrm{ZrO}_{2}$ in cladding structure (PCM-20 Test 8-1 RS) $\ldots \ldots \ldots$

III-4. Longitudinal section of fuel rod 24-1/2 inches from bottom of rod showing heavility oxidized cladding, with oxygen stabilized $\alpha$-Zr taking up most of cladding wall thickness

III-5. Longitudinal section of fuel rod at about 23 inches from bottom of rod showing absence of oxygen-stabilized alpha layer on inner surface of cladding adjacent to gap between fuel pellets (PCM-20 Test 8-1 RS) . . . . . . . . . . .

III-6. Transverse section of fuel rod showing fuel restructuring and cladding cracks at 24 inches from bottom of rod (PCM-20 Test 8-1RS)

IH-7. Longitudinal section of fuel rod showing fuel restructuring, cladding collapse, and cracks through cladding from 24-1/4 to 25 inches from bottom of fuel rod (PCM-20 Test $8-1$ RS) . . . . . . . . . . . . . . . . .

III-8. Cladding temperature axial profilc estimated from metallurgical examination of the PCM-20 Test 8-1 RS

111-9. Compositc transverse section of fuel rod slıowing microstrunture in clading

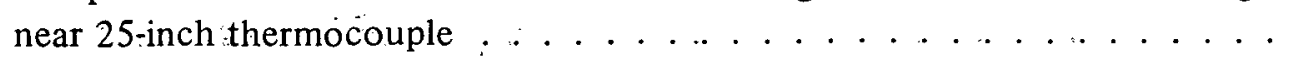

IH-10. Longitudinal section of fuel rod taken at about 27 inches from bottom of

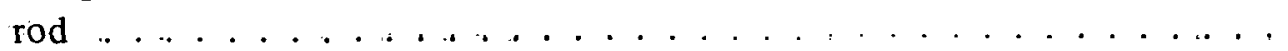

III-11. Transverse section of fuel rod 25-7/8.inches from bottom of fuel rod 
III-12. Composite photomicrograph across fuel rod at about $27-3 / 4$ inches from bottom of fuel rod showing fuel restructuring and cladding odixation . . . . 80

III-13. Transverse section of fuel rod showing fuel structure near center hole of fuel pellet, 27-3/4 inches from bottom of rod (PCM-20 Test 8-1 RS)

III-14. Transverse section of fuel rod 30-1/4 inches from bottom of rod $\ldots \ldots$. . . 82

III-1 5. Transverse section of fuel rod showing fuel structure near center hole of fuel pellet at 30-1/4 inches from bottom of rod (PCM-20 Test 8-1RS)

III-16. Fuel temperature vs. grain size for three grain growth kinetic equations

III-1 7. Transverse section of fuel rod showing fuel structure near outside diameter of fuel pellets $30-1 / 4$ inches from bottom of rod . . . . . . . 85

III-1 8. Fuel rod used in PCM-20 CHF Scoping Test . . . . . . . . . . . . . . 94

IV-1. Axial velocity profile of two-dimensional expansion junction . . . . . . . 102

IV-2. Pressure profile at expansion function $\ldots \ldots \ldots 103$

IV-3. Turbulent kinetic energy q protile . . . . . . . . . . . . . 104

IV-4. Turbulent viscosity $\mu_{\mathrm{t}}$ near wall region . . . . . . . . 105

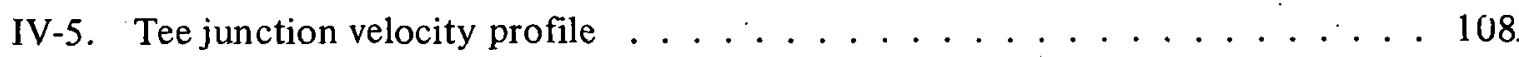

IV-6. Membrane swelling model . . . . . . . . . . . . . . 111

IV-7 Calculation of local axial curvature .............. 113

IV-8. Calculation of local circumferential curvature ............ 114

IV-9. Surface area and cladding thinning model $\ldots \ldots \ldots \ldots \ldots$

IV-10. Incremental deformation at node $\mathbf{i} \ldots \ldots \ldots \ldots$

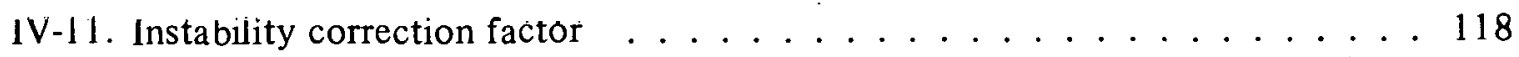

IV-12. BALLOON flow diagram ...................... 12U

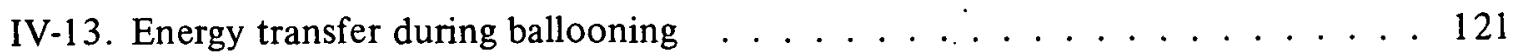

IV-14. Strain rate effect on cladding stress ... . . . . . . . . 123 
IV $=15$. Pressure and temperature history of burst samples $\ldots \ldots \ldots \ldots$

IV-16. Experimental vs. predicted ballooning shapes f . . . . . . . . . 127

IV-17. Total curcumferential elongation (TCE) as a function of temperature $\ldots \ldots 128$

IV-1 8. Effect of axial spacing on cladding displacement . . . . . . . . 129

IV-19. Effect of circumferential spacing on cladding displacement $\ldots \ldots \ldots$

IV-20. Effect of relative strength of central node on maximum swelling $\ldots \ldots \ldots 131$

IV-21. Influence of parameters on strain at failure $\ldots \ldots \ldots 132$

IV-22. Model predictions and measured values of zircaloy tube axial growth as a function of fast neutron fluence, irradiation temperature, cold work, and texture coefficient $\mathrm{f}_{\mathrm{z}} \ldots \ldots \ldots \ldots \ldots \ldots$

IV-23. Model predictions and measured values of growth of zircaloy tubes adjusted to a common texture coefficient of $\mathrm{f}_{\mathrm{z}}=0.05 \ldots \ldots \ldots$. . . . . 140

IV-24. Zircaloy growth versus square root of fast neutron fluence for data adjusted to a common tube texture coefficient of $f_{z}=0.05$ with linear least squares fits superimposed $\ldots \ldots \ldots . \ldots 14 . \ldots \ldots$

IV-25. Zircaloy growth versus square root of fast neutron fluence for data adjusted to a common tube texture coefficient of $\mathrm{f}_{z}=0.05$ and to a common temperature of $300^{\circ} \mathrm{C}$, with linear least squares fits superimposed . . . . . 142

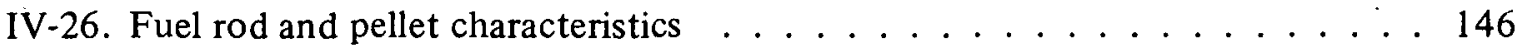

IV-27. Rod AJ dimensional profile . . . . . . . . . . . . . . 147

IV-28. Instrumented fuel assembly-226 . . . . . . . . . . . . . . . 148

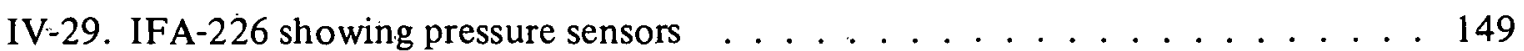

IV-30. IF A-226 showing cladding elongation sensors $\ldots \ldots \ldots \ldots$

IV-31. IFA-226 showing centerline thermocouple $\ldots \ldots \ldots \ldots 1$

IV-32. Initial rod elongation vs. rod peak power (zero burnup) $\ldots \ldots \ldots \ldots$

IV-33. Rod elongation vs. rod peak power (assembly average burnup $20 \mathrm{MWD} / \mathrm{MTM}$ ) . 1.54 
IV-34. Rod AM elongation vs. time (IFA-226 first power cycle) $\ldots \ldots \ldots \ldots$

IV-35. Effect of pellet-to-cladding gap size on fuel rod axial deformation $\ldots \ldots \ldots 6$

IV-36. Effect of fuel density on fuel rod axial deformation $\ldots \ldots \ldots 157$

IV-37. Elongation history of IFA-226 test pins from November 1971 through January $1972 \ldots \ldots \ldots \ldots$. . . . . . . . . . . . . . . . . . . . . . . . . .

IV-38. Elongation history of IFA-226 test pins from March 1972 through

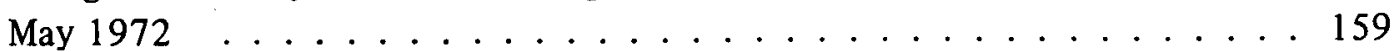

IV-39. Elongation history of IFA-226 test pins from Majy 1972 through July $1972 \ldots \ldots \ldots \ldots$

IV-40. Elongation history of IFA-226 test pins from July 1972 through August $1972 \ldots \ldots \ldots \ldots 16 \ldots \ldots \ldots \ldots$

IV-41. Elongation history of IFA-226 test pins from October 1972 through January $1973 \ldots \ldots$. . . . . . . . . . . . . . 162

IV-42. Elongation history of IFA-226 test pins from March 1973 through May $1973 \ldots \ldots \ldots \ldots . \ldots \ldots \ldots$

IV-43. Elongation history of IFA-226 test pins from June 1973 through July 1973

IV-44. Elongation history of IFA-226 test pins from August 1973 through September $1973 \ldots \ldots \ldots$. . . . . . . . . . . . 165

IV-45. Elongation history of IFA-226 test pins from December 1973 through January $1974 \ldots \ldots \ldots$. . . . . . . . . . . . . 166

IV-46. Elongation history of IFA-226 test pins from February 1974 through April $1974 \ldots \ldots \ldots \ldots 7$

IV-47. Fuel rod cross sections showing pellet cracking $\ldots \ldots \ldots$

IV-48. Fucl centcrlinc tempcrature vs. power during start-up . . . . . . . . . 169

IV-49. Fuel centerline temperature during 25 -hour period following start-up $\ldots \ldots$. 170

IV-50. Fuel rod AK centerline temperature vs. power for first power ramp $\ldots \ldots$. 171

IV-51. Rod AK fuel centerline temperature for first four full power ramps . . . . . 172 
IV-52. Rod AA fuel centerline temperature for first four full power ramps $\ldots \ldots \ldots$. . 173

IV-53. Fuel rod AA centerline temperature vs. power at extended burnup levels $\ldots 174$

IV-54. Fuel rod AO centerline temperature vs. power at extended burnup levels $\ldots 175$

IV-55: Fission gas pressure history $(1971-1973) \ldots \ldots \ldots \ldots \ldots$

IV-56. Fission gas pressure history $(1973-1974) \ldots \ldots \ldots$. . . . . . . 177

IV-57. Simplified Trojan plant model $\ldots \ldots \ldots 181$

IV-58. Simplified plant model vs. detailed plant model $\ldots \ldots \ldots \ldots 2$

IV-59. Correlation of LOCA analysis code predictions with Semiscale Mod-1

Test S-02-2 results .................... 185

IV-60. Correlation of LOCA analysis code predictions with Semiscale Mod-1

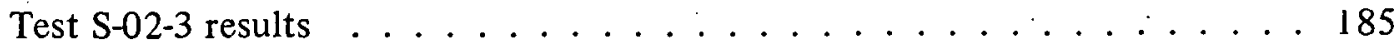

\section{TABLES}

II-1. Summary of WBFM Uncertainty Analysis . . . . . . . . . . . . 39

II-II. Summary of MFM Uncertainty Analysis . . . . . . . . . . . 40

II-III. Summary of DDAPS Uncertainty Analysis . . . . . . . . . . . . 40

II-IV. Summary of WBFM Voltage Insertion Calibration Uncertainty

Analysis .......................... 41

II-V. Summary of MFM Voltage Insertion Calibration System Uncertainty

Analysis ....................... 41

II-VI. . Summary of DDAPS Voltage Insertion System Uncertainty Analysis . . . 42

II-VII. Summary of 3-mV/V Full-Bridge Shunt Calibration Uncertainty

Analysis ....................... 43

II-VIII. Summary of $2-\mathrm{mV} / \mathrm{V}$ Full-Bridge Shunt Calibration Uncertainty

Analysis ...................... 43

H-IX. Summary of Half-Bridge Shunt Calibration Uncertainty Analysis _ . . . . 44 
II-X. Summary of Quarter-Bridge Shunt Calibration Uncertainty Analysis _ . . 44

II-XI. Summary of LLT Uncertainty Analysis . . . . . . . . . . . . . 45

II-XII. Summary of External Accelerometer Uncertainty Analysis . . . . . . . . . 46

II-XIII. Summary of LVDT Uncertainty Analysis . . . . . . . . . . . . . . 47

II-XIV. Summary of SPND Uncertainty Analysis . . . . . . . . . . . 48

II-XV. Summary of TIP Uncertainty Analysis . . . . . . . . . . . . . 48

II-XVI. Summary of Strainge Gage Uncertainty Analysis . . . . . . . . . . . 49

II-XVII. Summary of Standoff Absolute Pressure Uncertainty Analysis ........................ 50

II-XVIII. Summary of FFPT Uncertainty Analysis . . . . . . . . . . . . . . . 51

II-XIX. Summary Differential Pressure Transducer Uncertainty Analysis . . . . . . 52

II-XX. Summary of Experimental Temperature Uncertainty Analysis . . . . . . . . 54

II-XXI. Summary of DTT Turbine Uncertainty Analysis . . . . . . . . . . . . 55

II-XXII. Summary of DTT Drag Disk Uncertainty Analysis . . . . . . . . . . 56

II-XXIII. Summary of Pump Speed Uncertainty Analysis _ . . . . . . . . . . . 57

II-XXIV. Summary of Gamma Densitometer Uncertainty Analysis . . . . . . . . . 58

II-XXV, Cold Wall Delay Time . . . . . . . . . . . . . . 61

II-XXVI. Effective Steam Gencrator Piping Resistance _ . . . . . . . . . . 65

III-I. PCM-20 Test 8-1 RS Fuel Rod Centerline Temperature Calculated From Grain Size Measurements . . . . . . . . . . . . . . 87

III-II. Summary of Power Calibration Results for CHF Scoping Test . . . . . . . . 90

III-III. Summary of Experimental Results for CHF Scoping Test . . . . . . . . 92

III-IV. Thermal-Hydraulic Conditions Predicted at the Onset of CHF . . . . . . 93

IV-I. Base Line Cladding Parameters . . . . . . . . . . . . . 133 
IV-II. Measurements of Growth in Zircaloy Tubing . . . . . . . . . . . . . 137

IV-III. Zircaloy Growth Data as a Function of Cold Work and Fluence ........................ 143

IV-IV. Determination of Cold Work Coefficient . . . . . . . . . . 143

IV-V. Summary of Comparison of RELAP4 Predictions with Semiscale Mod-1 Core Test Data . . . . . . . . . . . . . . . . . . 184 


\title{
QUARTERLY TECHNICAL REPORT ON WATER REACTOR SAFETY PROGRAMS SPONSORED BY THE NUCLEAR REGULATORY COMMISSION'S DIVISION OF REACTOR SAFETY RESEARCH, JULY-SEPTEMBER 1976
}

\section{SEMISCALE PROGRAM}

\author{
J. O. Zane, Program Manager
}

\begin{abstract}
Documentation of results from the isothermal blowdown test series (Test Series 1) ${ }^{[I-1]}$ of the Semiscale Mod-1 Test Program has been completed and issued in the form of four topical reports. Typical results from the topical report covering phenomena related to emergency core coolant (ECC) injection ${ }^{[\mathrm{I}-2]}$ have been reported previously ${ }^{[\mathrm{I}-1]}$. Selected subjects from the remaining topical reports are discussed herein. The initial discussion presents an evaluation of the break flow phenomena that occurred during the isothermal test series and the methods which best predicted these phenomena. This discussion is extracted from the topical report describing the general thermal-hydraulic phenomena [I-3] that occurred during the tests. The flow regimes that occurred in the intact loop piping are discussed next. This discussion is extracted from the topical report describing an evaluation of the piping heat transfer, piping flow regimes, and steam generator heat transfer ${ }^{[\mathrm{I}-4]}$ that occurred during the tests. The final discussion presents an evaluation of the intact loop pump performance which was presented as part of the topical report on this subject $[I-5]$. These three subjects have been selected because they are believed to represent the information of greatest potential interest to readers of this quarterly report, and also because they cover some of the most significant results obtained from the Mod-l isothermal series of tests.
\end{abstract}

\section{BREAK F̄LOẄ}

\section{E. Cartmill}

Break flow phenomena that resulted from the simulated pipe rupture in each of the isothermal tests are of special interest because of their strong influence on overall system performance. Also, the fact that the measured flow was somewhat different from that predicted prior to the tests makes the subject significant in terms of its applicability to the analy tical models used to calculate break flow rates.

The phenomena occurring at both sides of the simulated break control the system depressurization rate and directly influence much of the phenomena occurring throughout the system. Initially, the fluid at the breaks is subcooled and the flow rates are high. When the pressure at the break falls to the saturation pressure of the fluid, choking at the break commences. Choking may occur at one break earlier (at the pump side of the break for a $200 \%$ cold leg break) than at the other and in so doing affect the system flow rates and directions. During saturated blowduwn, the flow rates throughout the system are also affected by the conditions at the breaks competing with the conditions at the pump. 
An accurate measurement of the flow properties at each side of the break is thus necessary to properly interpret results at other places within the system. The measurements recorded at the breaks include fluid temperature, fluid pressure, fluid density, momentum flux, and differential pressure across the break assembly.

Each side of the break is simulated by a converging-diverging nozzle, with the measurements located just upstream of the converging portion of the nozzle. Evaluation of these measurements and comparison of the Semiscale data with predictions obtained using various correlations provide a better understanding of the subcooled and saturated blowdown phenomena occurring at the breaks.

\subsection{Subcooled Blowdown}

The flow rate and duration of subconled flow at the break nozzles is influenced by the pressure and the initial degree of subcooling at the break. After nupture, the break pressure dropped from the initial pressure $(2,250$ psig for the cold leg break) to the saturation pressure of 926 psia between 20 and 100 milliseconds depending on the side of the break considered. Since the pump and steam generator simulators have large hydraulic resistances, the pressure at the break downstream of the simulators (pump side) drops quickly to the saturation condition, whereas the pressure at the vessel side of the break drops more slowly. The subcooled blowdown pressure for Test S-01-4A is illustrated in Figure I-1 which shows the pressure at the vessel side of the break dropping to the saturation pressure in 100 milliseconds, whereas the pressure at the pump side of the break dropped to the saturation pressure in 20 milliseconds. The flow rates at the break were large during the subcooled portion of blowdown due to the acceleration of the fluid. Figures I-2 and I-3 show the subcooled flow rates at the vessel and pump sides of the break for the $200 \%$ cold leg break configuration. The flow rate drops fairly rapidly when the fluid begins to decelerate measurably.

RELAP4 [ ${ }^{[-6]}$ calculations have been used, in addition to the data, to investigate the subcooled portion of blowdown and its influences on system behavior. The subcooled critical break flow models used in RELAP4 to calculate the subcooled break flow rates were the Henry model $[\mathrm{I}-7]$ and the momentum model $[\mathrm{I}-6]$. The Henry critical flow model is based on a momentum balance, and the critical mass flux is obtained in tabular form as a function of stagnation pressure and enthalpy which are taken from the volume iust upstrcam of the choking plane. Since the Henry model was originally developed for saturated blowdown, the tabular information is provided by an "extended" Henry table of values applicable to subcooled conditions. Once the mass flux is obtained, a contraction cocfficient (Inultiplier) is used to account for the flow losses encountered in the fluid going through the converging-diverging nozzles at the break. The multiplier value of 0.6 was determined to be appropriate for the nozzle configuration used in the Semiscale system. The momentum model is based on the solution to the one-dimensional momentum equation in conjunction with the continuity equation. Figure I-4 shows the subcooled blowdown pressure at the vessel side of the break for Test S-01-4A and includes RELAP4 calculations in which the two different subcooled break flow models were used. 


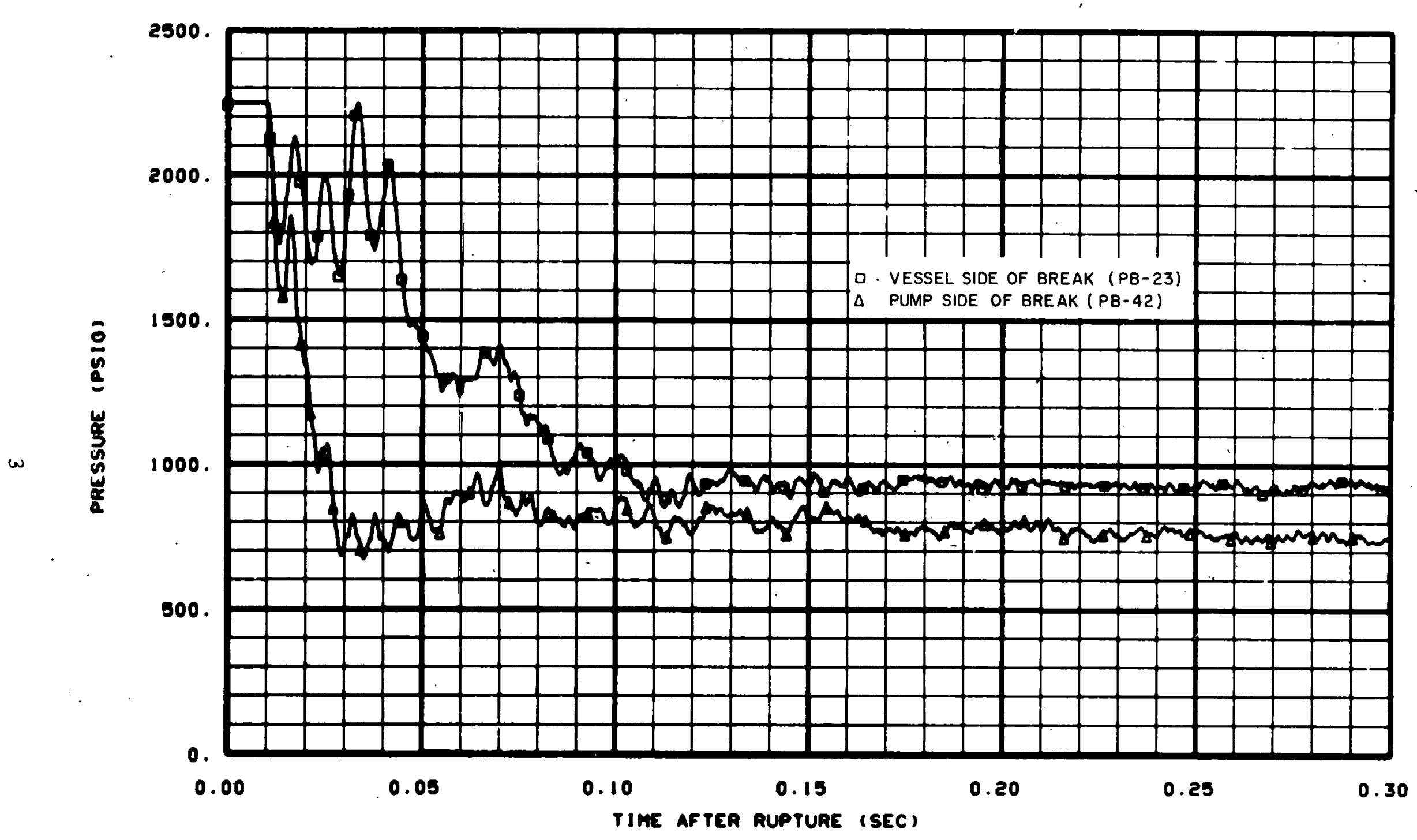

Fig. I-1 Subcooled blowdown pressure at vessel and pump sides of break - Test S-01-4A. 


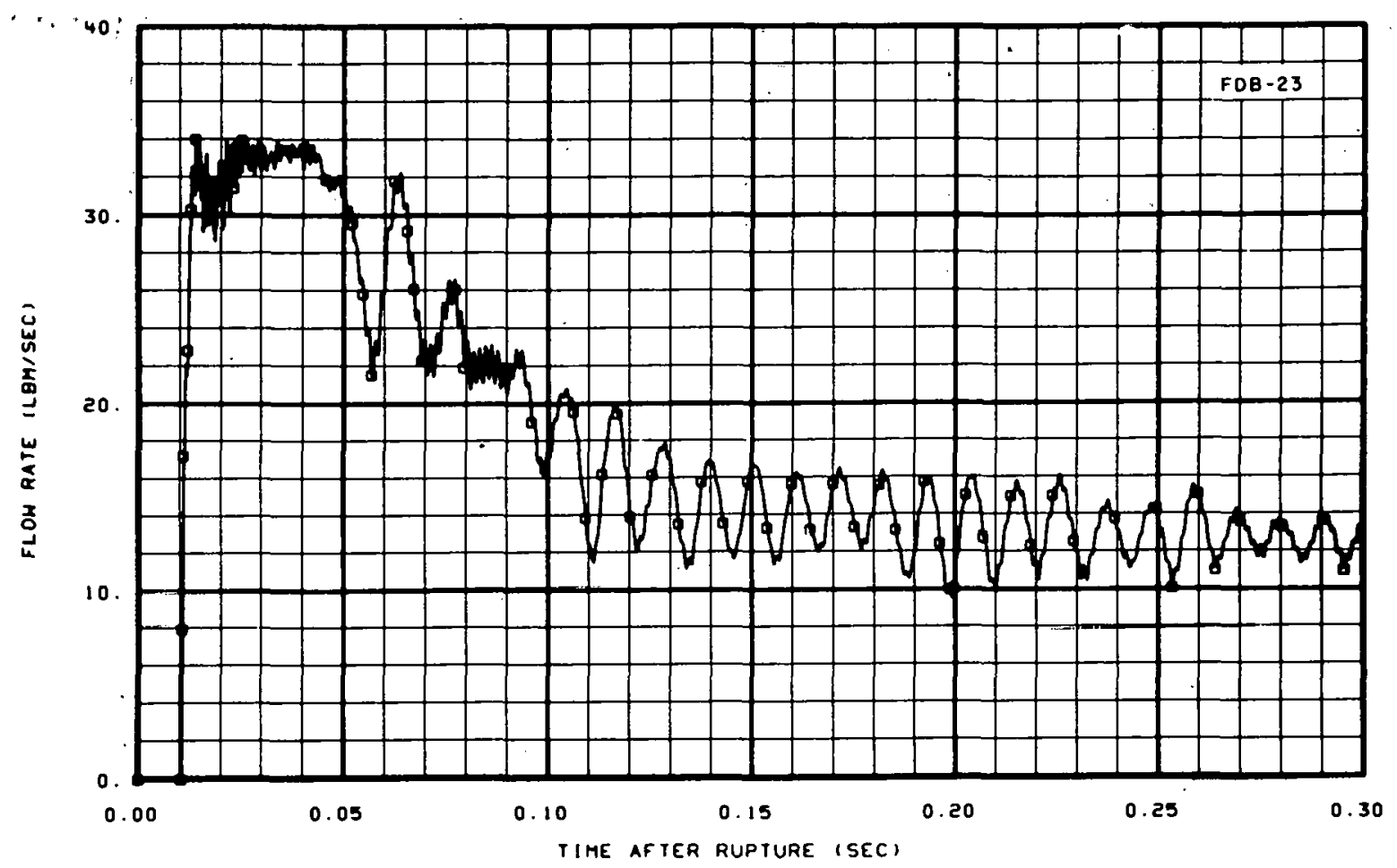

Fig. 1-2 Subcooled blowdown flow rate at vessel side of break - Test S-01-4A.

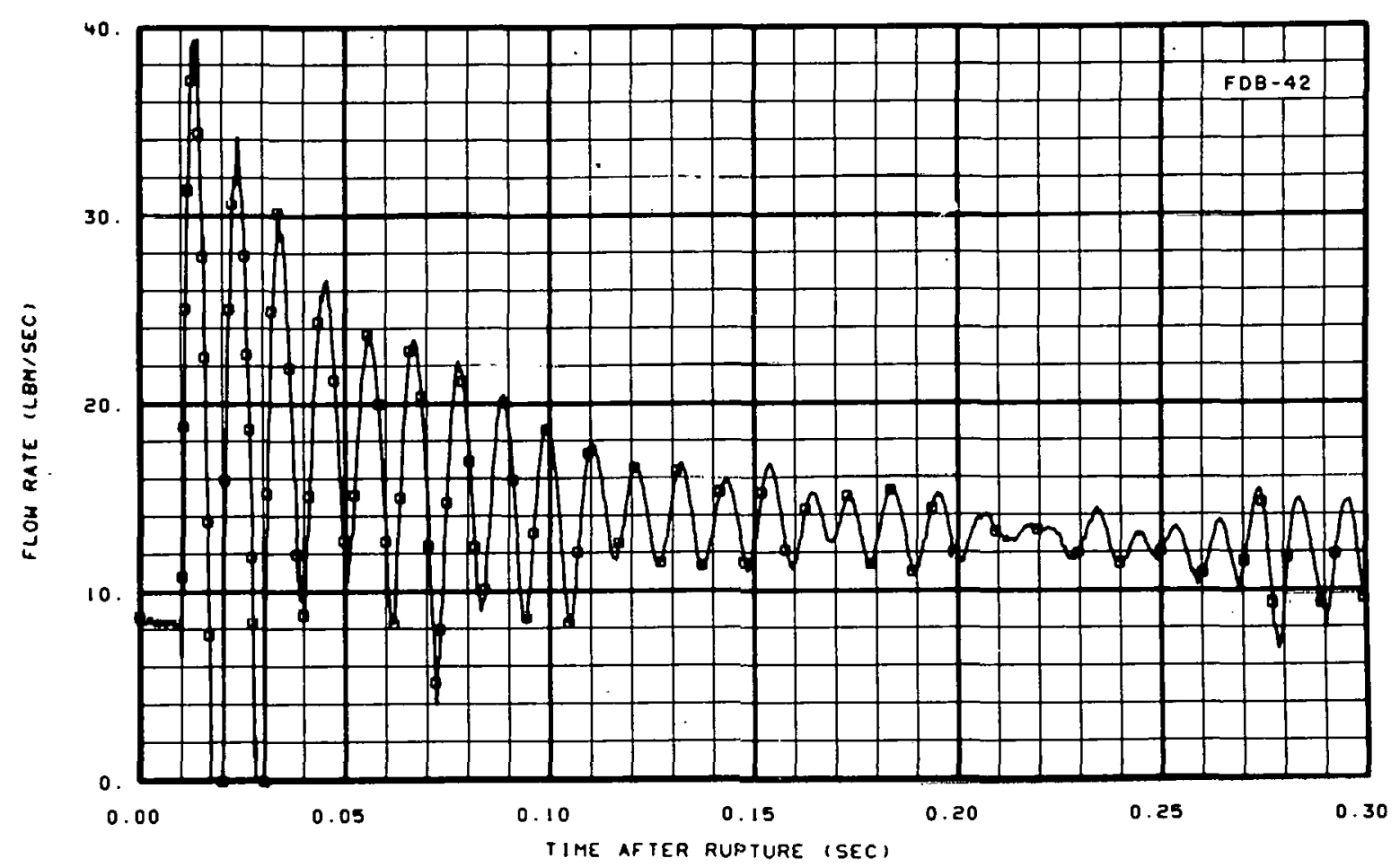

Fig. I-3 Subcooled blowdown flow rate at pump side of break - Test S-01 4 A. 


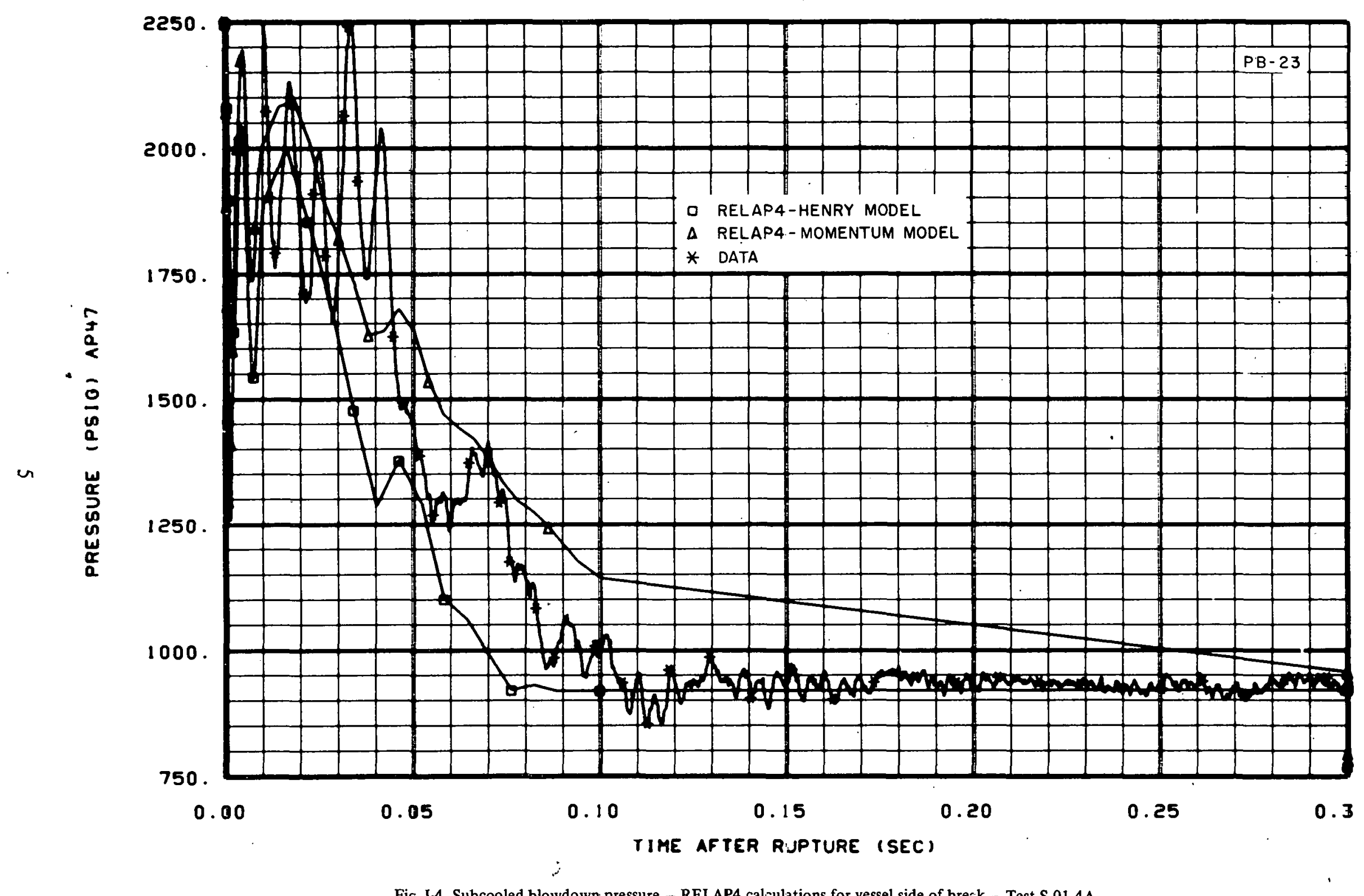

Fig. I4 Subcooled blowdown pressure - RELAP4 calculations for vessel side of break - Test S-01-4A. 
The subcooled critical break flow model used in RELAP4 which most closely matched the data was the Henry model. RELAP4 calculates the pressure for the first 60 milliseconds of subcooled blowdown reasonably well. The momentum model, however, overpredicted the pressure, causing a slightly lower depressurization rate and a higher calculated flow rate than existed with the data. Figure I-5 shows that the results of the flow rate obtained utilizing the Henry model more closely match the physical phenomena occurring at the break than those obtained through use of the momentum model. A more nearly accurate determination of the density, resulting from a closer evaluation of the depressurization rate, accounts for the fact that the Henry model calculates values closer to the data than does the momentum model.

Calculations from the two RELAP4 models also aid in determining the influence of subcooled break flow on system response. The influence that the subcooled critical break flow had on system response is observed within the core region. Figure I-6 compares the RELAP4 calculated core flow rates obtaincd through use of the previously mentioned break flow models with the data for Test S-01-4A. The momentum model predicts an extended period of subcooled flow and results in negative flow through the core during the subcooled blowdown, whereas the Henry model predicts a shorter period of subcooled flow and results in positive flow during the same time interval. The measured flow was positive. The core fluid behavior is therefore very sensitive to the critical break flow model used during subcooled blowdown, indicating the fluid conditions at the break strongly influence the core fluid behavior during subcooled blowdown.

\subsection{Saturated Blowdown}

The saturated blowdown flow rate is influenced by the pressure and fluid density at the break nozzles. The relatively small saturated blowdown depressurization rate (compared to the subcooled depressurization rate) shown in Figure I-7 and the corresponding large reduction in flow rate shown in Figure I-8 indicate the change from subcooled to saturated flow. The slower depressurization rate for saturated blowdown (Figure 1-7) is due to smaller flow rates out the break and the degree of fluid flashing within the system. As was the case during the subcooled blowdown, the resistances in the simulators cause flow differences between the two break locations. The saturated blowdown history at the break indicates that $65 \%$ of the fluid leaving the system left the vessel side of the break for the $200 \%$ cold leg break configuration (35\% flowed from the pump side of the break). The lower flow restrictive path out the vessel side of the break, as compared with that out the pump side, accounts for the difference in the percentage of the fluid out each break. In the hot leg break $(100 \%)$ configuration, about $50 \%$ of the fluid leaving the system went out each side of the break. "The difference in the relative distribution of the flow out the breaks for the hot and cold leg break configurations is attributed to differences in the hydraulic resistance of the paths the flow takes to get to the break.

RELAP4 calculations have also been used to investigate the saturated portion of blowdown to aid in understanding the influence of the break on system fluid behavior. The saturated critical break flow models used in RELAP4 to calculate the saturated break flow rates were the Moody critical flow model ${ }^{[I-8]}$ and the sonic model ${ }^{[I-6]}$. The Moody critical 


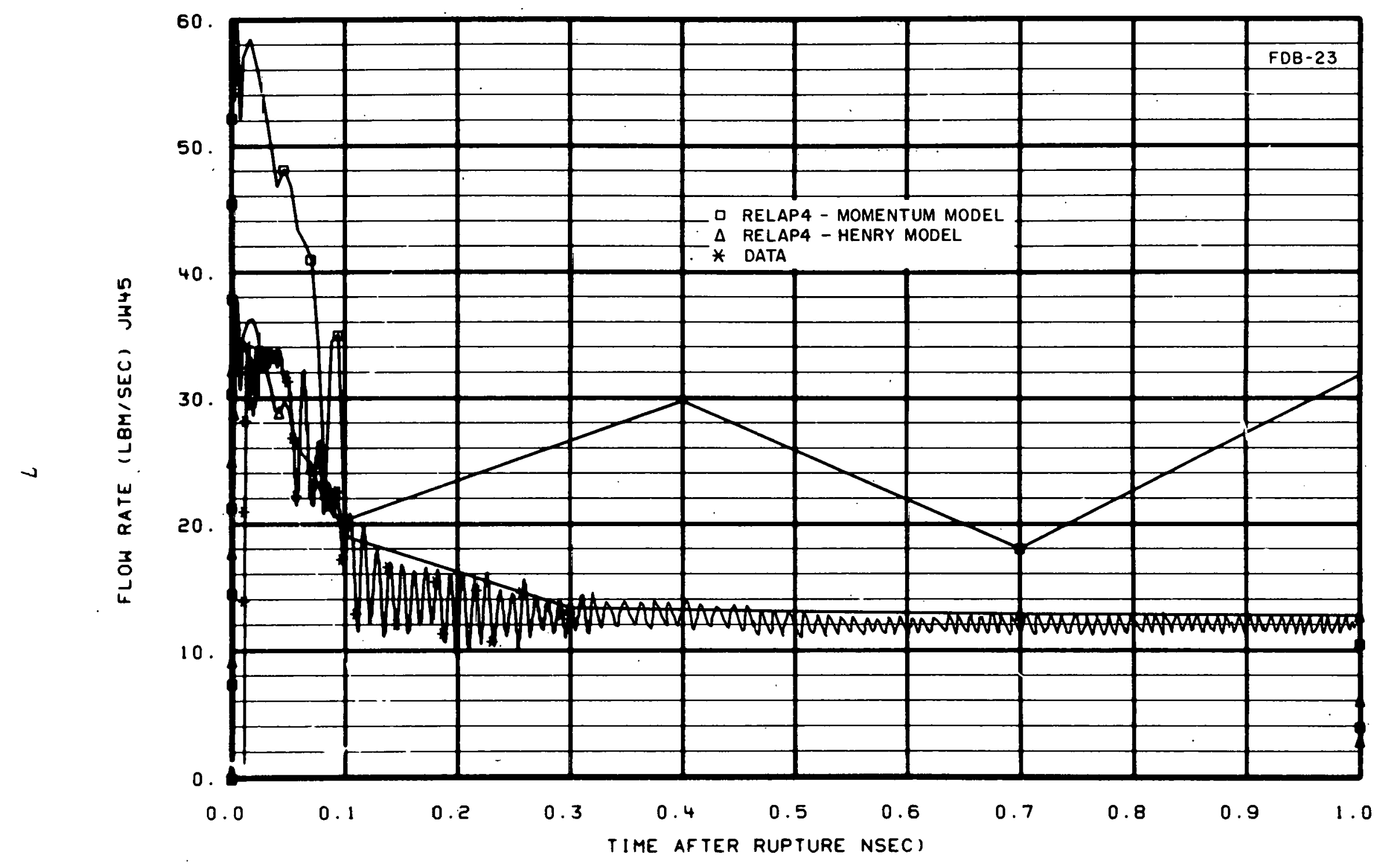

Fig. I-5 Subccioled blowdowin flow rate - RELAP4 calculations for vessel side of break - Test S-01-4A. 


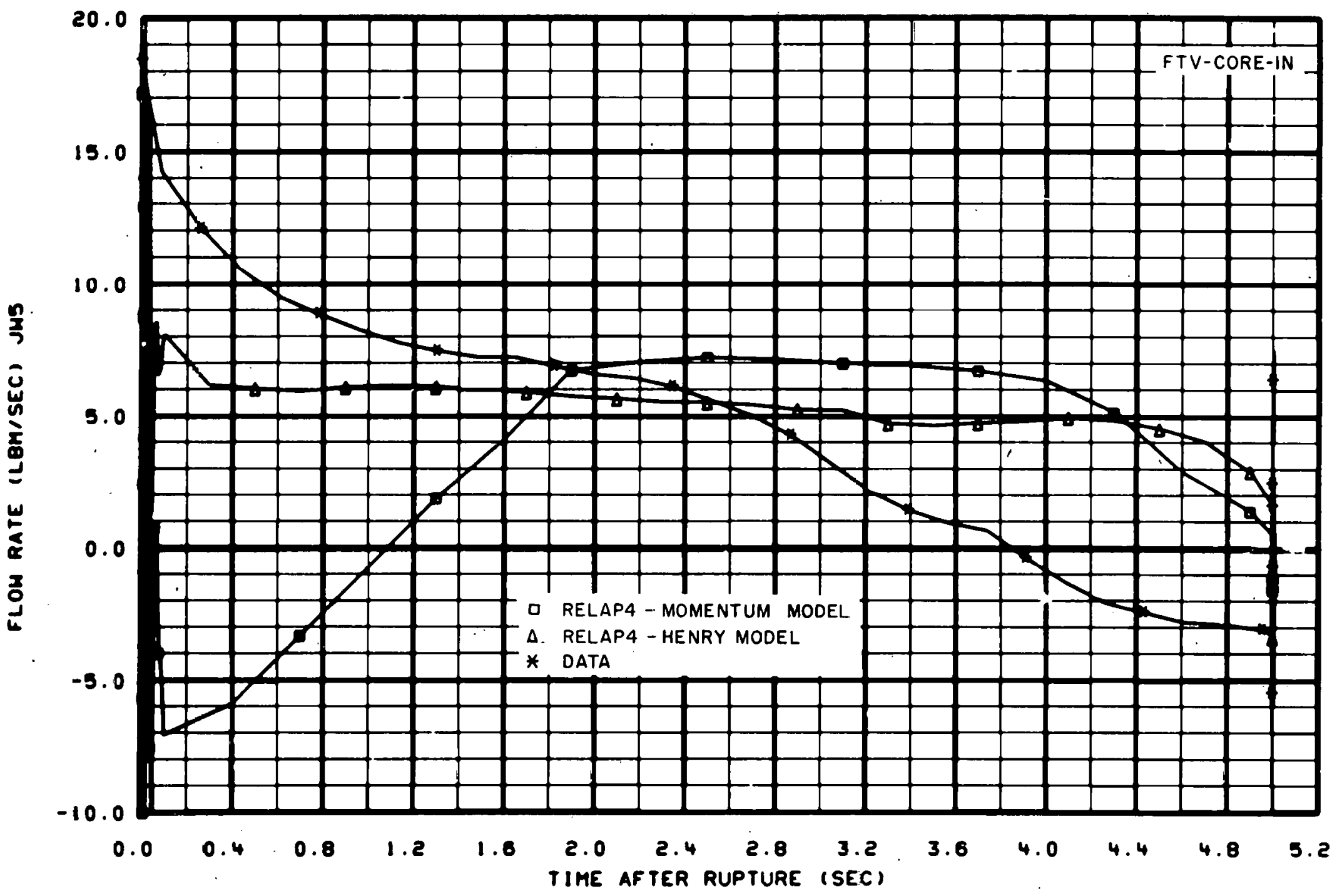

Fig. I-6 Subcooled blowdown core flow rate - RELAP4 calculations for Test S-01-4A. 


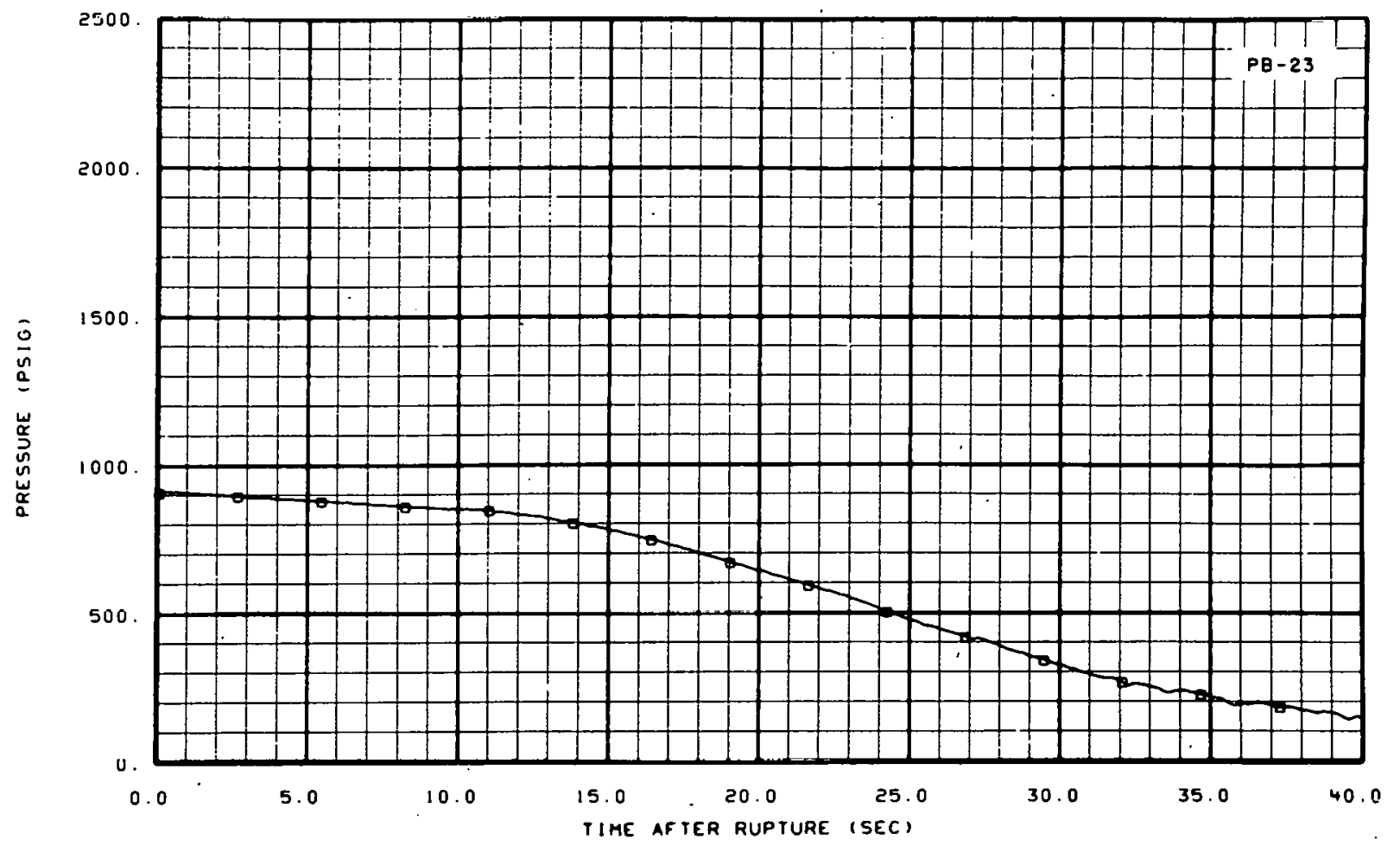

Fig. I-7 Saturated blowdown pressure at vessel side of break - Test S-01-4A.

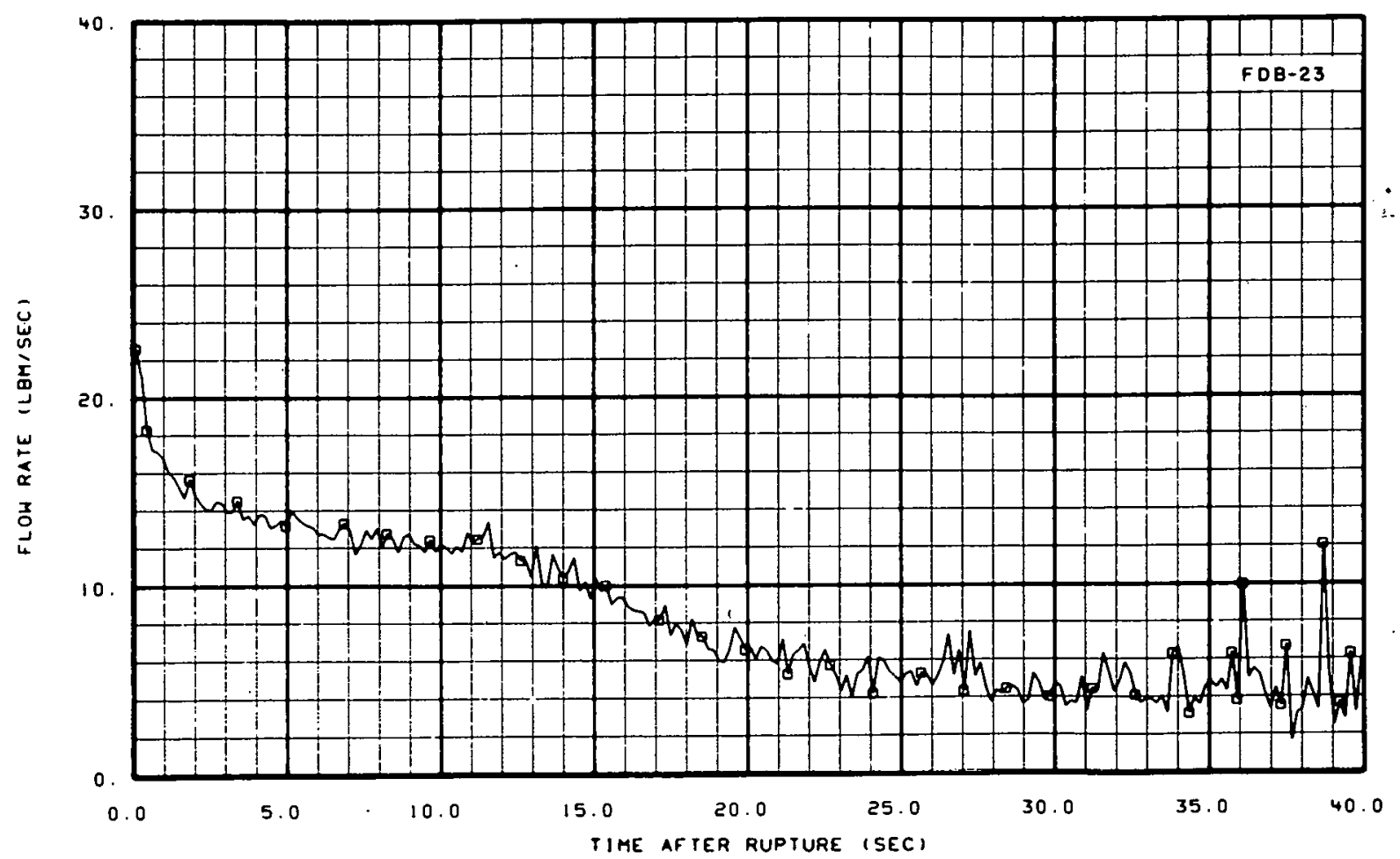

Fig. I-8 Saturated blowdown flow rate at vessel side of break - Test S-01-4A. 
flow model is based on an energy balance using the first law of thermodynamics and takes into account slip within the two-phase mixture in calculating the break flow rate. The critical mass flux is obtained in tabular form as a function of stagnation pressure and enthalpy which are taken from the volume just upstream of the choking plane. The sonic critical flow model in RELAP4 calculates the sonic velocity at the break by means of the steam tables assuming homogeneous flow, using the specific volume and internal energy of the volume just upstream of the choking plane. Once the sonic velocity is determined, the mass flux is calculated through knowledge of the geometry at the choking plane. The principal difference between Moody's approach and the sonic velocity approach is that Moody's work takes into account slip at the break, whereas the sonic approach does not take slip into account.

RELAP4 calculations obtained using the Moody and sonic models, shown in Figure I-9, are in general agreement with the data. Both RELAP4 calculations of the system pressure were higher than the data for the first 15 seconds of blowdown. The higher RELAP4 pressure resulted in higher calculated flow rates out the vessel side of the break as shown in Figure I-10. In Figure I-10 the use of the Moody critical tlow model in RELAP4 (using an 0.6 contraction coefficient) resulted in considerable overprediction of the flow rate at the vessel side of the break during saturated blowdown, whereas the sonic model appears to predict the saturated blowdown fairly well. The fact that the sonic value more closely matches the Semiscale Mod- 1 isothermal tests series experimental data implies that the fluid is homogeneous as it passes through the break nozzles. In fact, this implication is supported by the experimental configuration. The drag disc used to measure the momentum flux at the break has a target which is 0.406 inch in diameter and which is centered in a pipe 1.338 inches in diameter. The target is positioned just 1 inch upstream of the converging section of the break nozzles, and is thus just upstream of the choking plane. The target is thought to have disturbed the flow immediately upstream of the choking plane and to have caused homogenization of the flow at the throat of the break nozzle.

The break flow rates for the $100 \%$ hot leg break tests were also studied to determine the effect of the break size and configuration on break flow response during saturated blowdown. Because of the smaller break size (and hence lower depressurization rate) used for the hot leg break configuration, the fluid flow was not homogenized as for the larger $200 \%$ break tests and resulted in more flow "bubble separation". A slip model was therefore incorporated into the RELAP4 code for the parts of the system vertically oriented to account for the additional bubble rise phenomena. Figure I-11 shows the flow from the vessel side of the break for Test S-01-1B and compares the experimental flow with that calculated by RELAP4 with and without the slip model included. The Henry and Moody critical break flow models with a 0.6 break tlow multiplier were used for the subcooled and saturated portions of blowdown, respectively. The break flows in the hot leg break configuration for Tests S-01-1 and S-01-1B were underpredicted from 6 to 30 seconds. The RELAP4 calculation with slip appears to follow the data better. Although the slip model was not applied to horizontal piping and therefore did not calculate slip in the piping directly upstream of the simulated break nozzle, the calculation of density in vertical 


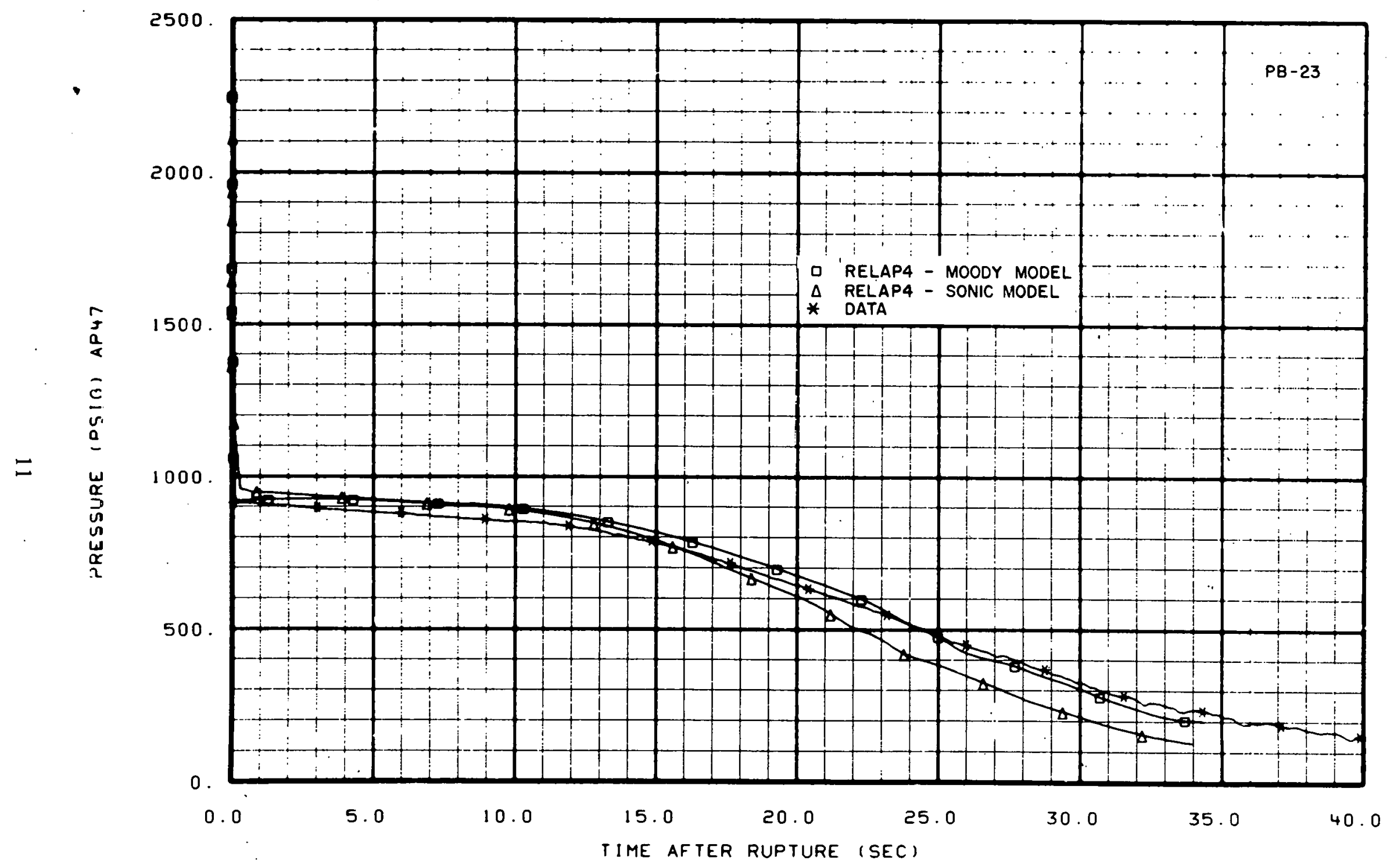

Fig. I-9 Comparison of experimental and calculated saturated blowdown pressures at vessel side of break - Test S-01-4A. 


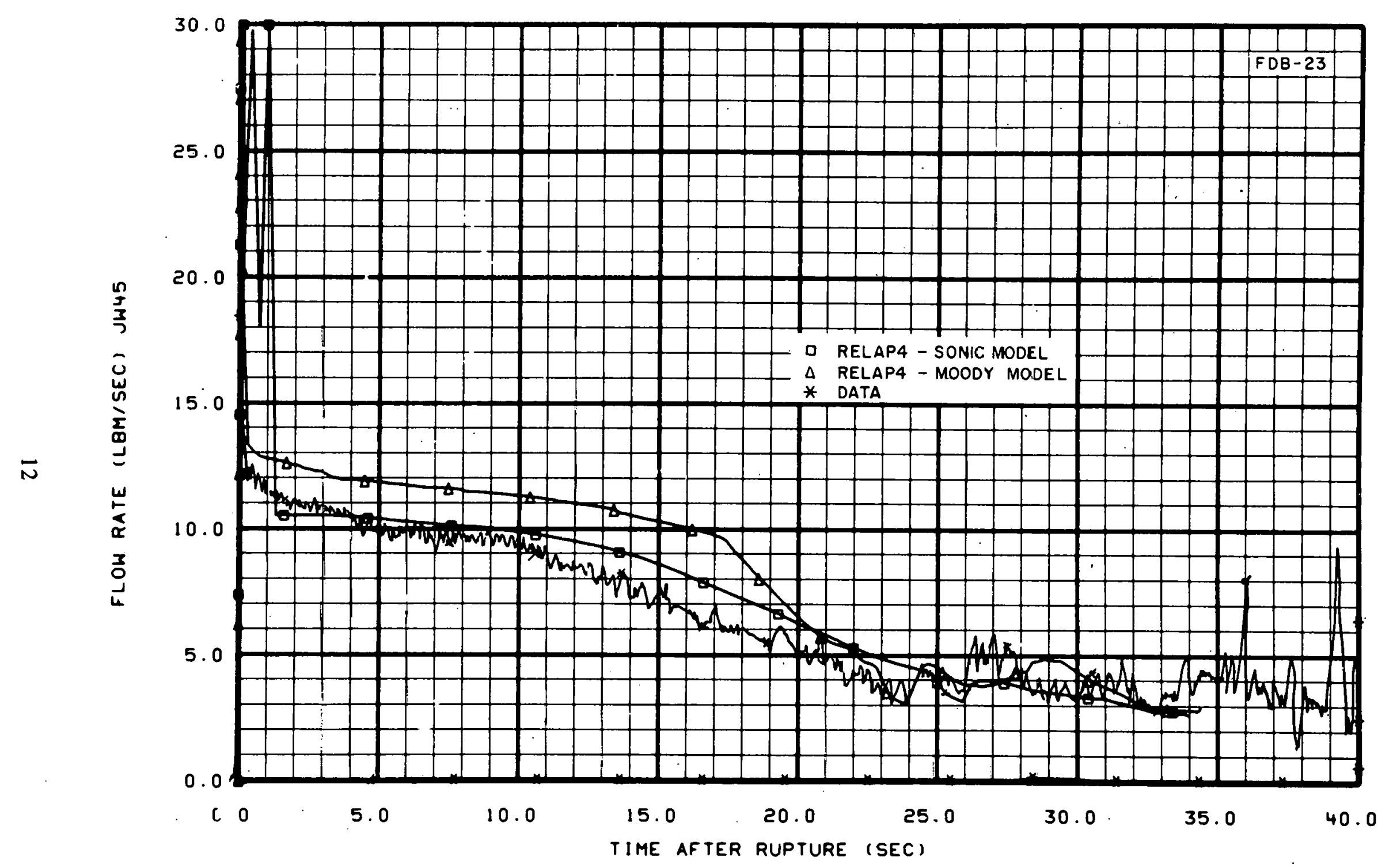

Fig. I-10 Comparison of experimental and calculated saturated blowdown flow rates at vessel side of break - Test S-01-4A. 


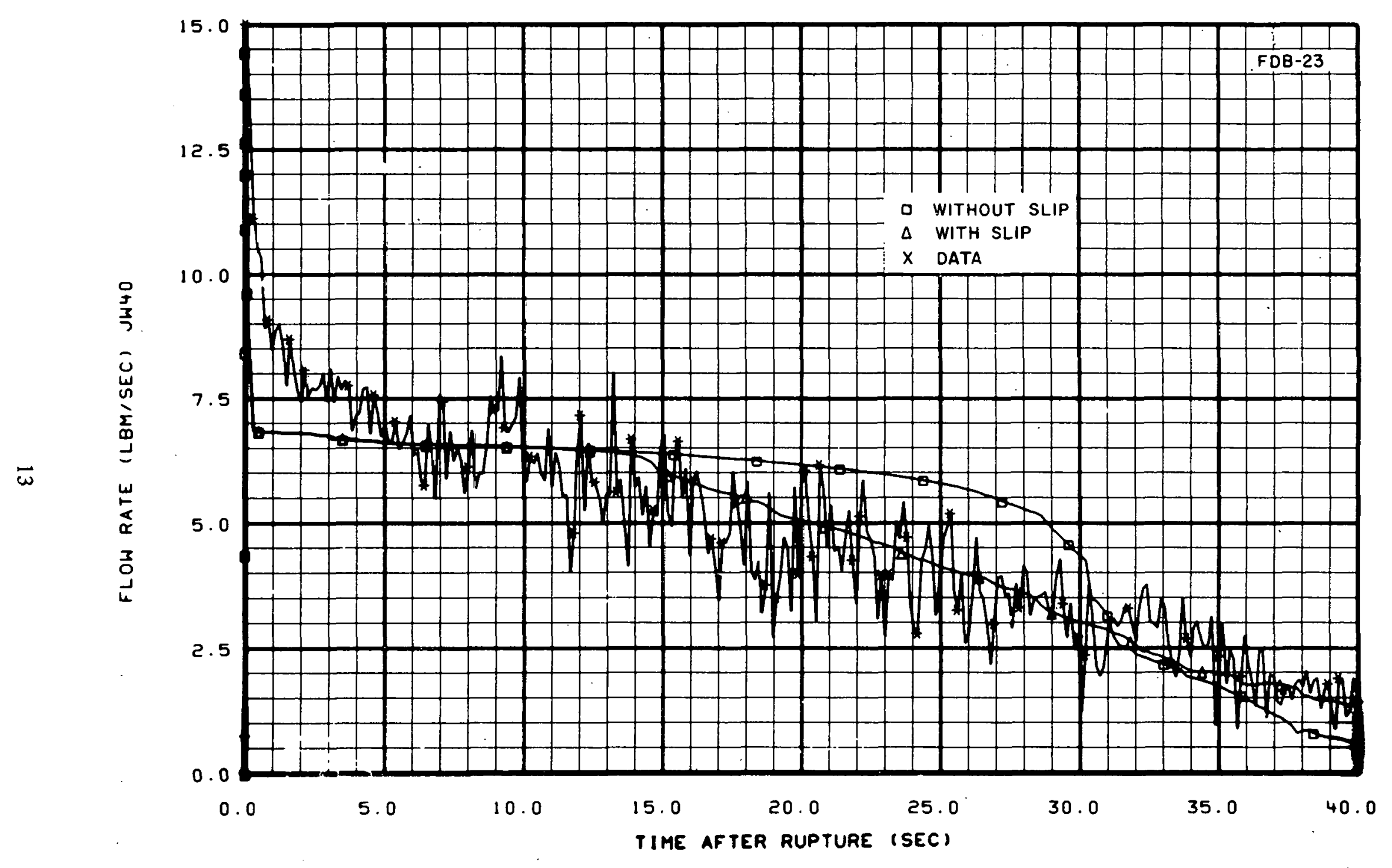

Fig. I-1 1 Flow rate at vessel side of break - comparison of calculations with and without slip (Test S-01-1B). 
regions improves in RELAP4, thus improving the density calculation of the fluid coming into the pipe leading to the break ${ }^{[a]}$.

The principal difference in flows from the vessel side of the break for the $100 \%$ hot leg versus the $200 \%$ cold leg break is shown in Figure I-12. As expected, the smaller break test exhibited a lower flow rate during the blowdown process until about 45 seconds at which time a negative flow occurred in Test S-014A. The back flow (reverse flow) through the vessel side of the break lasted from 45 to 62 seconds after rupture. For several tests with ECC injected into the cold leg of the intact loop (Tests S-01-4, S-01-4A, and S-01-5), the large amounts of condensation taking place in the broken loop cold leg and inlet annulus caused the pressure to be lower than that existing within the pressure suppression tank. Condensation resulted from the ECC passing from the intact loop cold leg into the inlet annulus and bypassing directly to the broken loop cold leg rather than proceeding down the downcomer. The downcomer and intact loop cold leg vessel inlet instrumentation shows very little reverse flow during this time, indicating that the reverse flow of fluid through the vessel side of the break is principally condensed within the inlet annulus.

The principal influence of the break fluid conditions on system response during saturated blowdown occurs within the core region. Knowledge of core fluid properties during blowdown is important to blowdown analysis in that high core flow rates and low fluid qualities result in high energy removal from the core.

The general response of the core flow for saturated blowdown during several of the $200 \%$ cold leg break isothermal tests is shown in Figure I-13. The core flow data from the $200 \%$ cold leg break tests (with the exception of those from Test S-01-2 which had a high intact loop flow resistance) are negative by 5 seconds after rupture, and flow stagnation occurs at about 15 seconds and is followed by a further negative flow until the end of blowdown. The core flow direction during blowdown was influenced by the conditions at the breaks. Although this influence cannot be shown directly from the data, a study of RELAP4 calculations aids in an understanding of the influence of the break conditions on core flow. The RELAP4 calculation using the sonic critical break flow model indicates, as shown in Figure I-14, negative flow through the core early in time (less than 1 second). However, the data in Figures I-13 and I-14 show the flow to be positive for the same time interval, and negative flow not evident until after 4 seconds. The RELAP4 calculation obtained through use of the Henry and Moody critical break flow models for subcooled and saturated blowdown, respectively, not only gave positive flow during saturated blowdown but fairly closely matched the test data. The results presented in Figure I-14 show that the calculation of core flow is very sensitive to the critical break flow model used in RELAP4, which implies that the break conditions have significant influence on core fluid behavior.

[a]. The slip model was also tried in a $200 \%$ cold leg break RELAP4 calculation, but a similar improvement in the density calculation was not observed for the large break, indicating that the more rapid depressurization and the larger flow rates resulted in a generally more homogeneous fluid in the broken legs. 


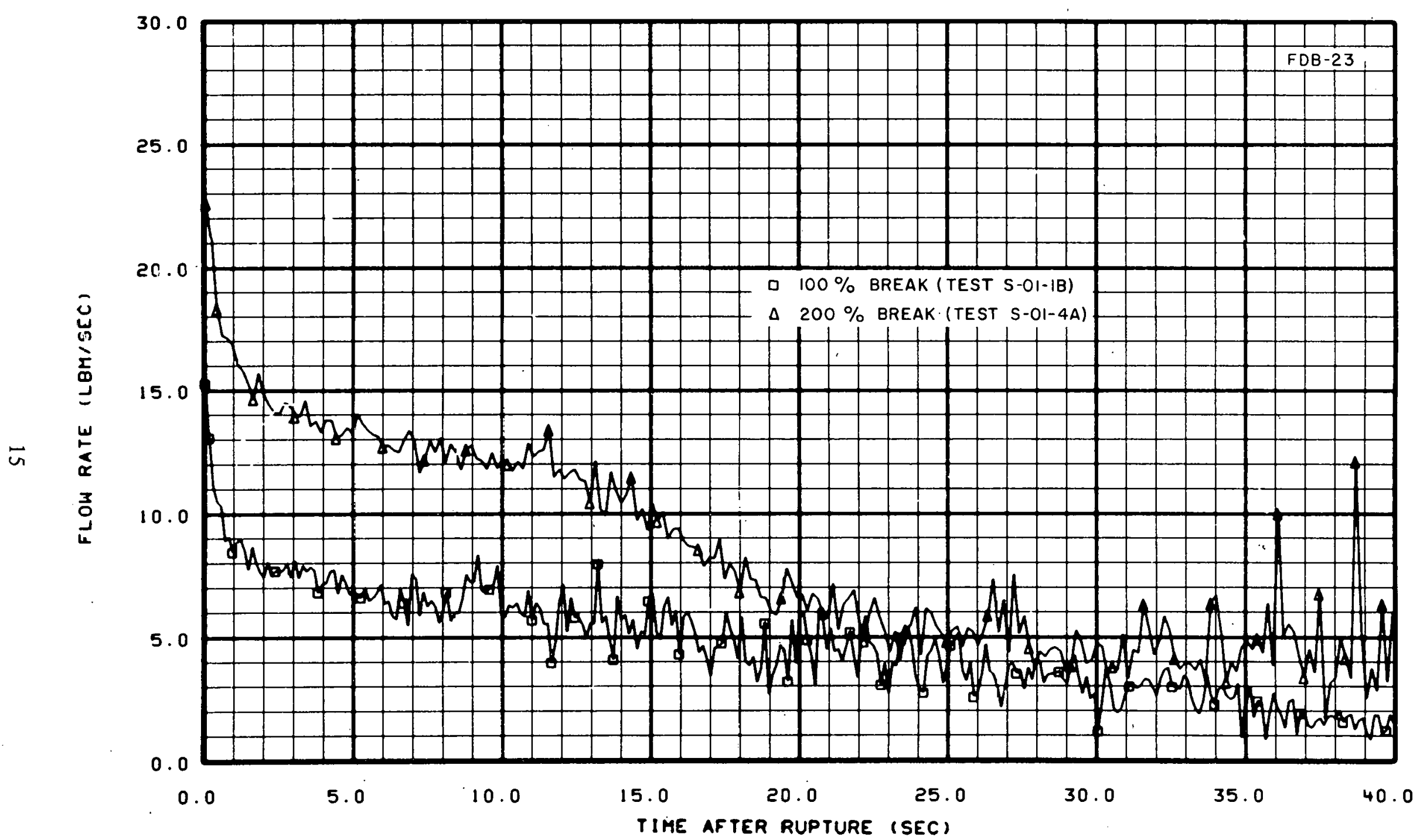

Fig. I-12 Flow rate at vessel side of break - comparison of results of $100 \%$ break (Test S-01-1B) and of 200\% break (Test S-01-4A). 


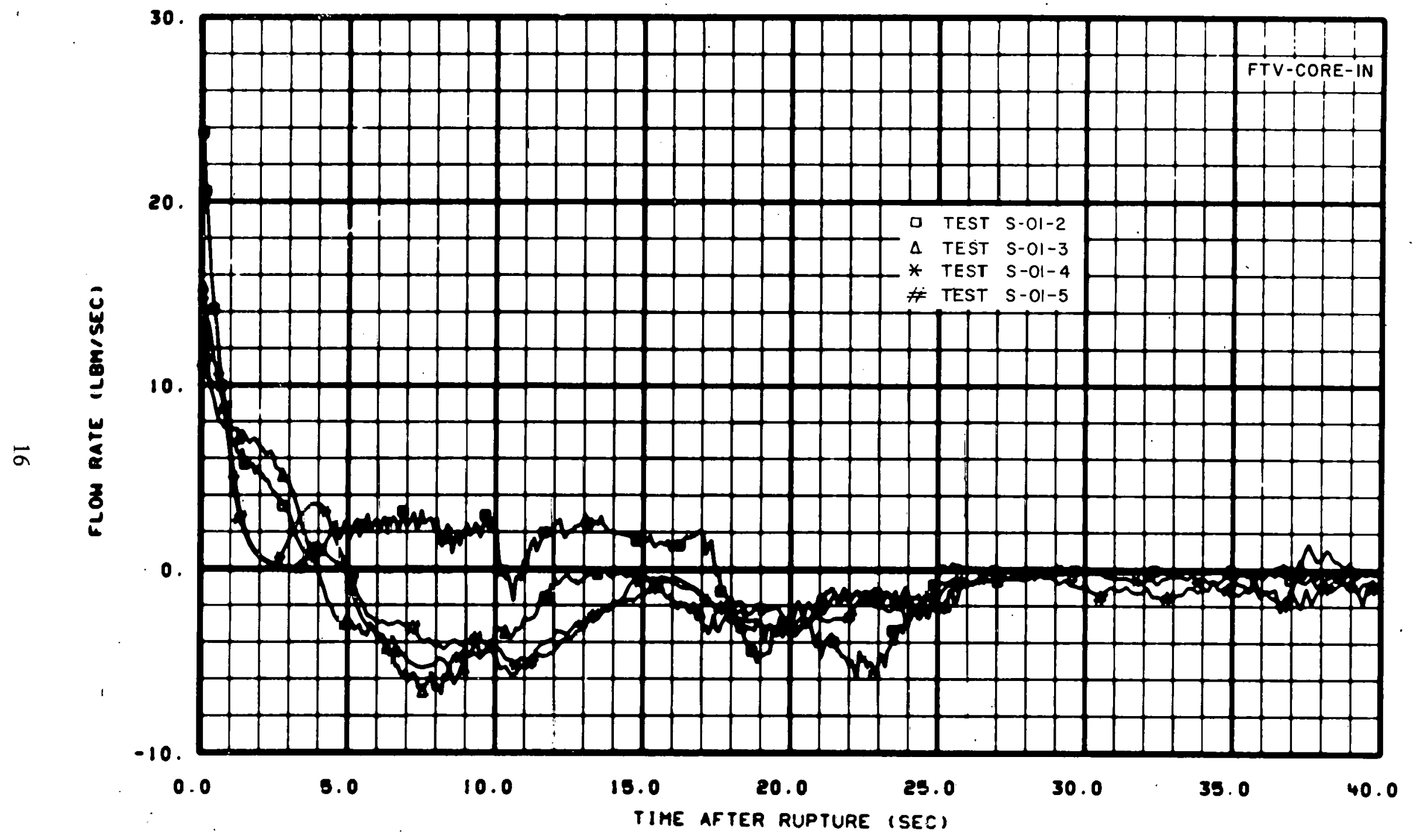

Fig. 1-13 Flow rate at entrance to core - Test $S-01-2, S-01-3, S-01-4$, and S-01-5. 


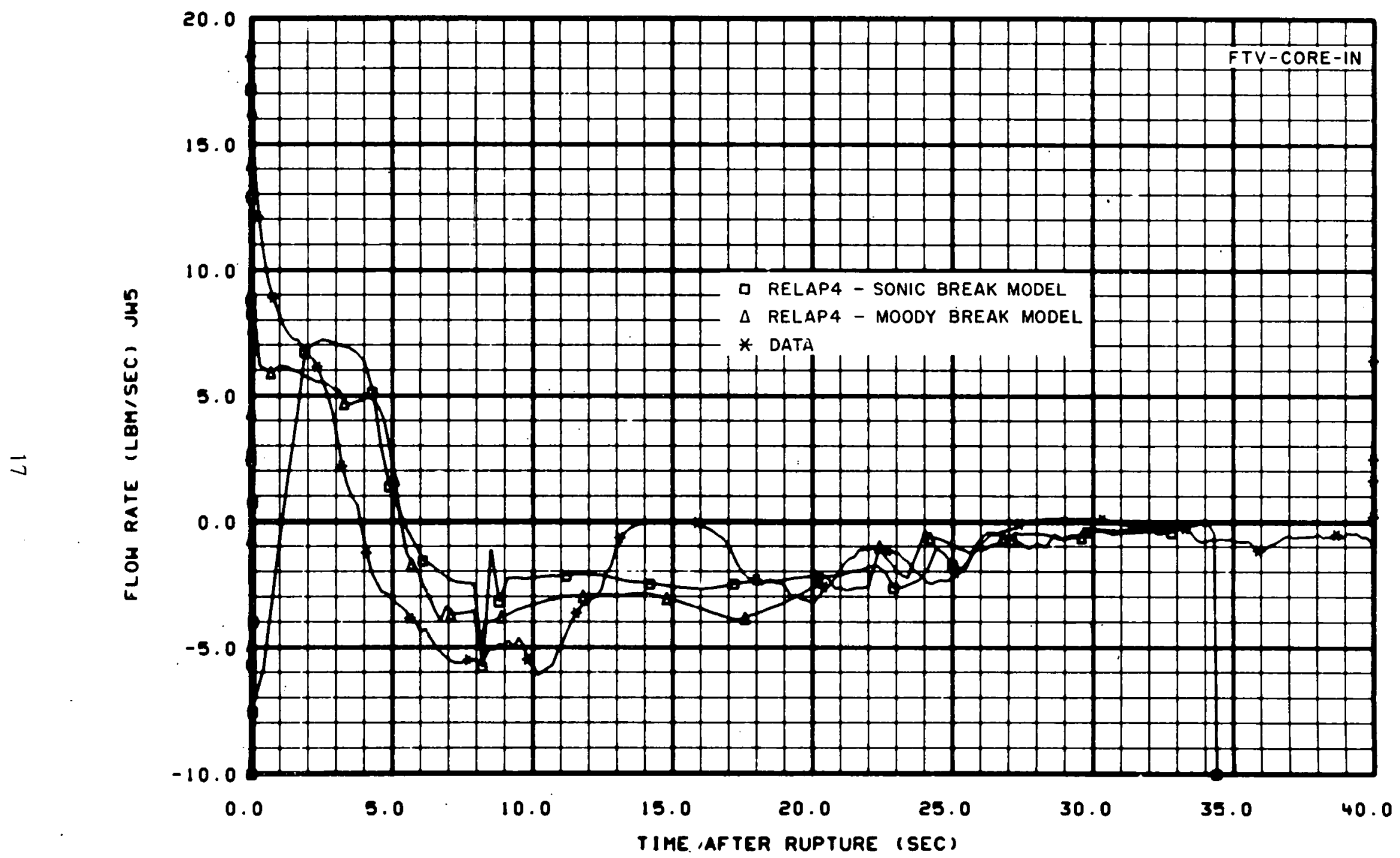

Fig. I-14 Flow rate at entrance to core - comparison of RELAP4 results with Test S-01-4A data. 
The influence of the break conditions on core flow was also studied for the hot leg break configuration. The core fluid flow direction for the $100 \%$ small break was positive for the entire blowdown period as shown in Figure I-15. The hot leg break configuration employed in Test S-01-1B resulted in break conditions which caused a slower discharge rate from the downcomer through the broken loop cold leg. This in turn resulted in a higher pressure in the downcomer region than in the core. In addition to the break influencing core behavior, the pump also aided significantly in causing the continued positive flow through the core. Furthermore, the resistive path from the core through the vessel side of the break was much smaller than that which existed for the cold leg break tests, allowing a strong positive flow through the core during the early stages of blowdown.

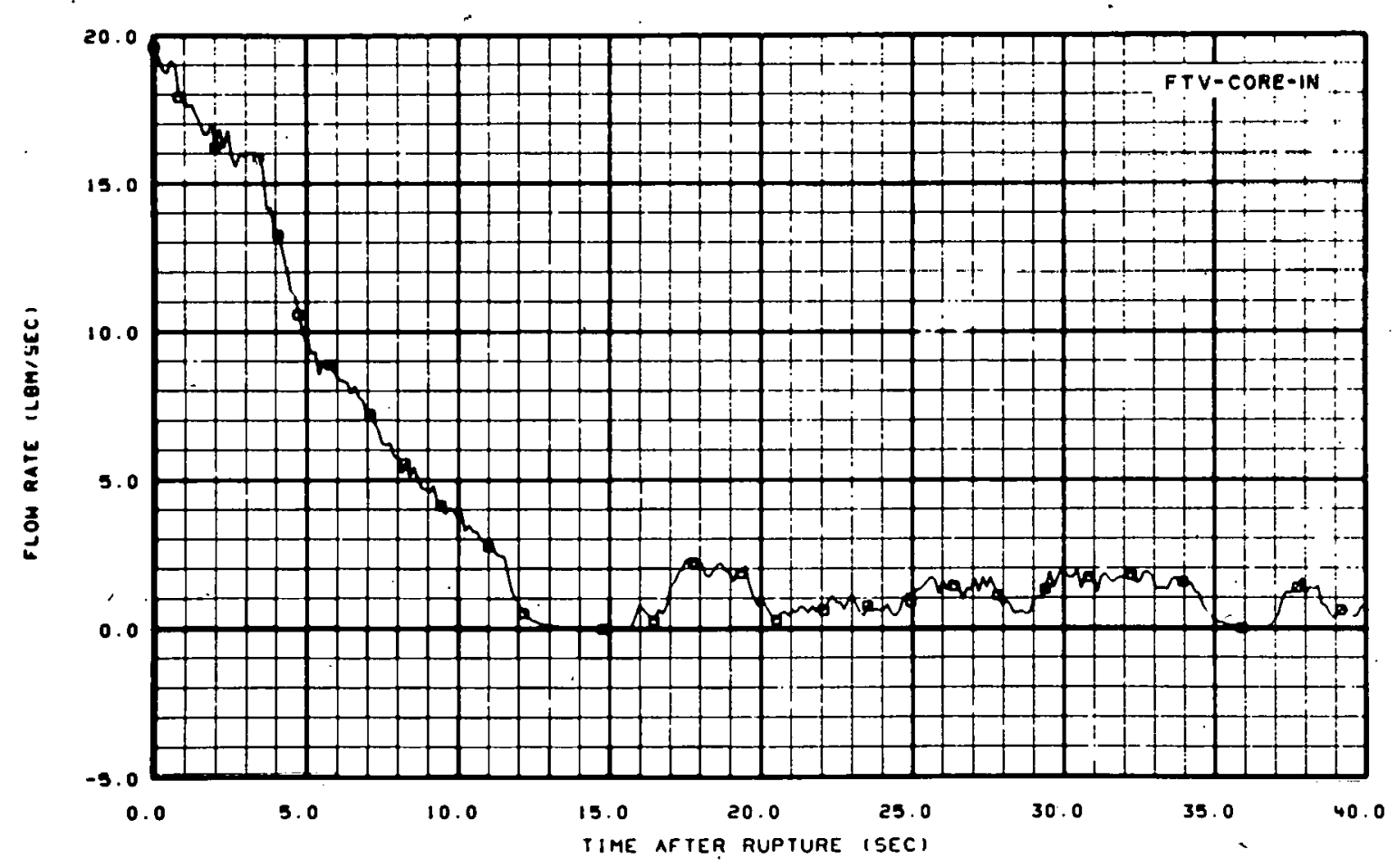

Fig. I-15 Flow rate at entrance to core - Test S-01-1B.

\subsection{Break Flow Summary -}

Analysis of the phenomena occurring at the simulated brcak leads to the conclusion that the break fluid conditions control the depressurization rate and directly influence the core fluid response during blowdown.

The core fluid behavior is very sensitive to the critical break flow model used during subcooled blowdown, indicating that the fluid conditions at the break stongly influence the core fluid behavior during subcooled blowdown. A more nearly accurate determination of the fluid density at the breaks, resulting from a closer evaluation of the depressurization rate, accounts for the fact that the Henry subcooled critical flow model calculates values closer to the data than do the other models used. 
The saturated fluid flow at the breaks was determined to be homogeneous as it passed through the break nozzles, accounting for the fact that the RELAP4 sonic critical break flow model most closely matched the data for saturated blowdown.

\title{
2. PIPING FLOW, REGIMES IN THE SEMISCALE GEOMETRY
}

\author{
R. T. French
}

The pressure and heat transfer that occur in the intact loop during a simulated LOCA can be strongly influenced by the primary fluid flow regimes existing in the pipes. In order to examine the application of steady state flow maps to the evaluation of transient data, flow regime histories werc calculated in strategic pipe sections in the Semiscale Mod-1 intact loop using a method suggested by Govier and Omer ${ }^{[I-9]}$. The calculated flow regimes were compared with flow regimes estimated from density measurements in the intact loop. The calculations required inputs of mass flow rate, liquid and steam phase densities, and the fluid, void fraction. An iterative method was required to obtain the flow regimes. This method is documented in Reference 14 . The calculated flow regime data were then plotted using a new flow map proposed by Mandhane, Gregory, and Aziz ${ }^{[1-10]}$ which has gas and liquid superficial phase velocities as the coordinate axes. The map was compared to the data of Baker $[\mathrm{I}-11]$, Hoogendoorn $[\mathrm{I}-12]$, Govier and Omer $[\mathrm{I}-9]$, and others, pertaining to air-water flow in horizontal pipes and has been shown ${ }^{[I-10]}$ to be in better agreement with the existing air-water data than any other. The study performed by Mandhane et al indicated that the effect of pipe diameter on flow regime boundaries was negligible when superficial phase velocities are used as the coordinate axes. The map has been adjusted to account for steam-water physical properties and a temperature of $530^{\circ} \mathrm{F}$ using a method suggested by Mandhane et al $[\mathrm{I}-10]$.

The flow map of Mandhane, and for that matter all others, has been developed on the basis of measurements of steady flow and by visual observation of the flow pattern. Although no visual observation of flow pattern is possible in the Semiscale Mod-1 system, and steady flow certainly does not exist during the transient, an evaluation of the applicability of the existing flow maps to a transient thermal-hydraulic fluid field can be made by comparing the predicted flow regimes with the measured densities from the paired horizontal and vertical densitometers located at the intact loop hot leg and intact loop cold leg.

Tests S-01-4A and S-01-1B were evaluated to determine variations in flow regimes existing at the intact loop hot leg vessel outlet and cold leg vessel inlet as a result of varying break configurations.

In the following figures, the flow regime at a given location for a given test was calculated two seconds into the transient and at each two-second interval thereafter. The resulting points were connected by a dotted line. The reader should not, however, conclude that the flow regime transition between two plotted points moves precisely along the dotted line. The total time into the transient is indicated in parentheses beside each point. 
The calculated flow regimes in the intact loop hot leg vessel outlet (Spool 1) during Tests S-01-1B and S-01-4A are shown in Figure I-16. The same flow regimes, including order of transition, were calculated for both tests with the exception that slug flow is indicated at one point during Test S-01-4A and not indicated during Test S-01-1B. The flow regime history calculated for the $200 \%$ cold leg break (Test S-01-4A) did significantly lead, in terms of time into transient, that calculated for the $100 \%$ hot leg break (Test S-01-1B). This difference between the two flow patterns was expected because the larger $200 \%$ break allows a faster system depressurization resulting in higher qualities, larger velocities, and hence faster flow regimc transition. The vertical and horizontal densitometer measurements obtained at the intact loop hot leg vessel outlet (Spool 1) during Tests S-01-4A and S-01-1B are shown in Figures I-17 and I-18, respectively. The vertical and horizontal density measurements were subjectively evaluated against each other in conjunction with mass flow rate and fluid velocity data to determine the flow regimes. The subjective requirements for

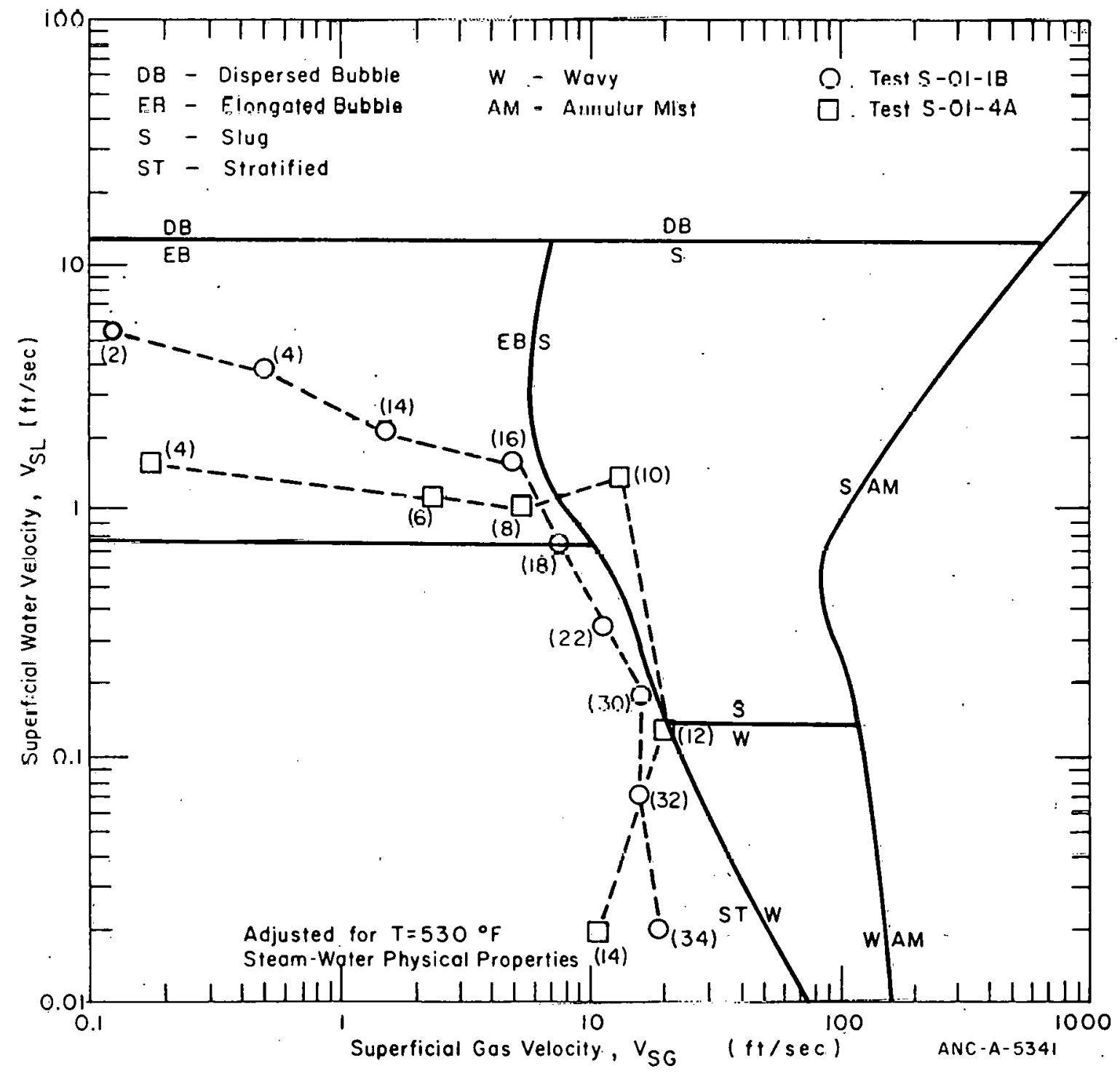

Fig. I-16 Flow regimes in the intact loop hot leg vessel outlet. 


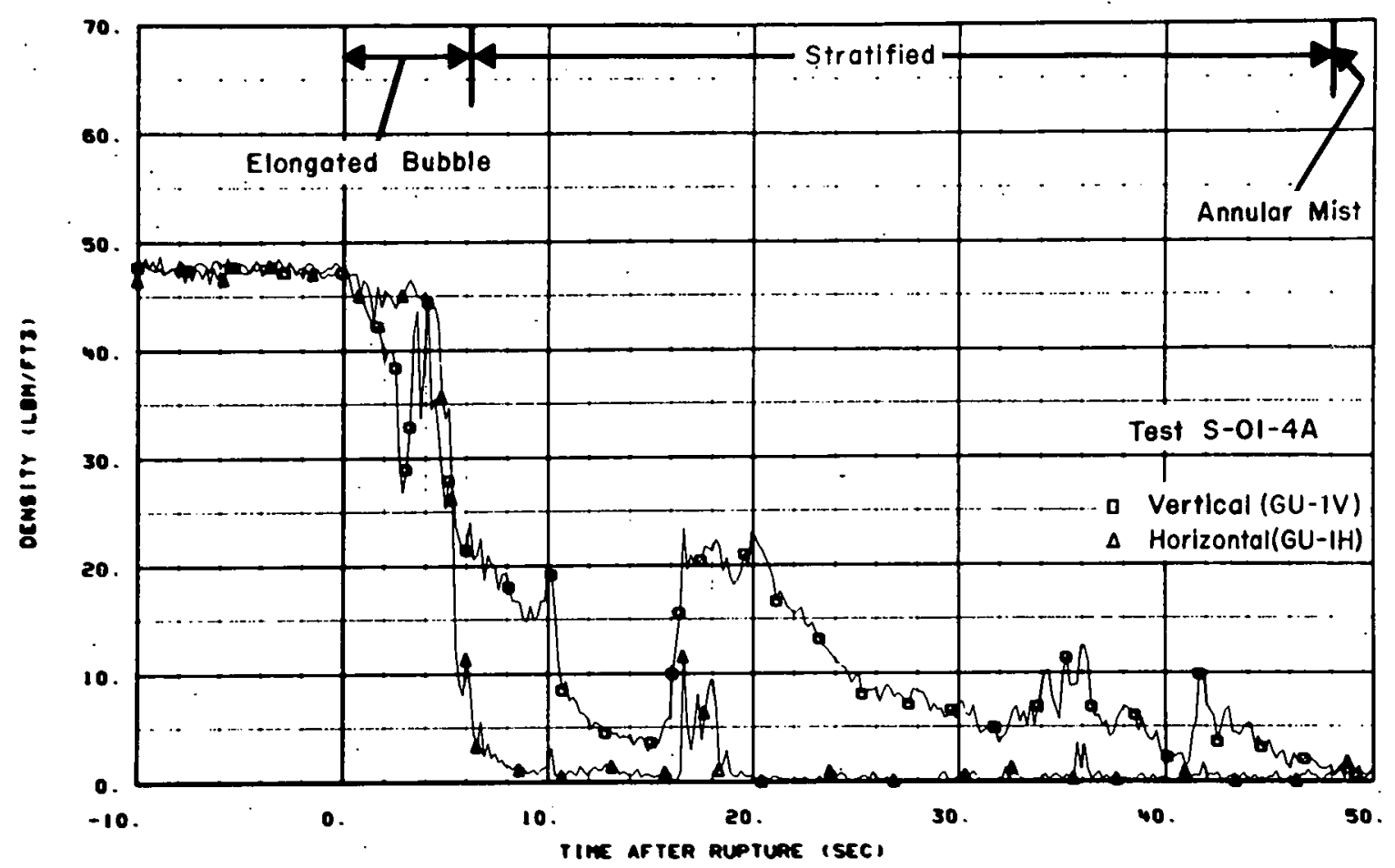

Fig. I-17 Flow regimes shown by the vertical and horizontal density measurements at the intact loop hot leg vessel outlet - Test S-01-4A.

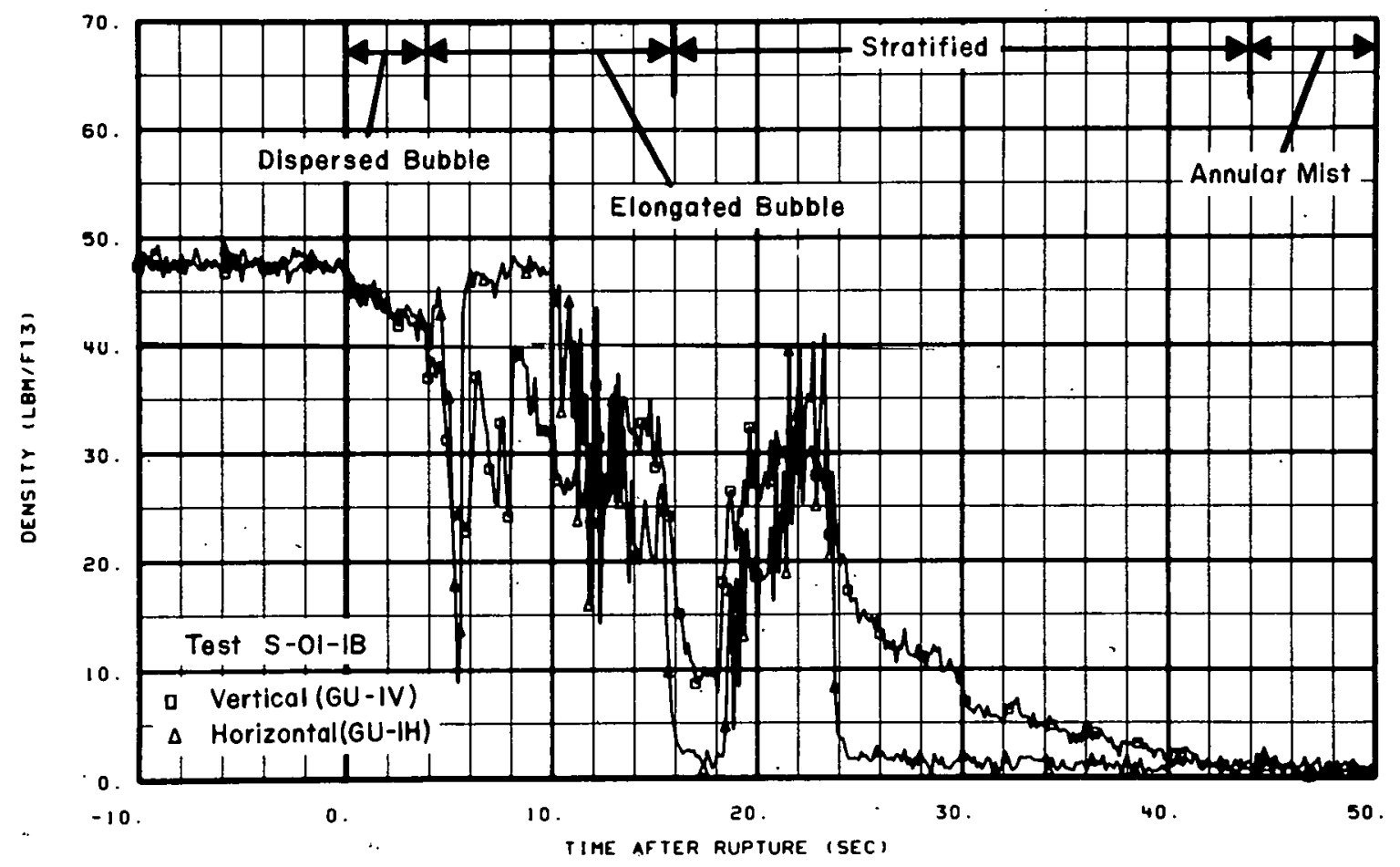

Fig. 1-18 Flow regimes shown by the vertical and horizontal density measurements at the intact loop hot leg vessel outlel - Test S-01-1D. 
specifying the possible flow regimes were as follows: (a) dispersed bubble flow was assumed early in the transient when both densitometers measured approximately the same value; (b) elongated bubble flow was assumed when the horizontal density measurement was significantly higher than the vertical measurement and only oscillations of minor magnitude were occurring in the horizontal density measurements; (c) slug flow was assumed when oscillations in both horizontal and vertical density measurements were of fairly large magnitude; (d) stratified flow was assumed when the vertical density measurement was higher than that recorded by the horizontal density measurements and both measurements were non-oscillatory; (e) annular-mist flow was assumed late in the transient when both density measurements recorded essentially the same value which was generally less than $5 \mathrm{lb}_{\mathrm{m}} / \mathrm{ft}^{3}$. No differentiation was made between wavy flow and stratified flow. The preceding assumptions regarding interpretation of the horizontal and vertical density measurements were modified, if required, on the basis of measured fluid velocity dat.a. For example, if the density measurements indicated elongated bubble flow, the velocity data were checked and if the velncity was zero or near zero, a stratified flow rcgimc was assumed.

Three flow regimes are clearly shown in Figure I-17 to occur at the intact loop hot lcg vessel outlet during Test S-01-4A. Elongated bubble flow occurs for about the first 6 seconds into the transient. Stratified flow exists from 6 to 48 seconds into the transient after which time the annular mist pattern is established. This evaluation agrees with the calculated flow regimes presented in Figure I-16 quite well with the exception of the point calculated 10 seconds into the transient when slug flow is indicated.

In general, the calculated flow regime results are in good agreement with the density data (shown in Figure I-18) obtained during Test S-01-1 B at the intact loop hot leg vessel outlet. An exception is that dispersed bubble flow is indicated by the density measurements for the first 4 seconds of the transient but was not indicated in the calculations. The transition from elongated bubble to stratified flow was calculated to occur at about 118 seconds and was indicated by the densitometers at about 16 seconds. Transition to annular mist is shown by the denisty information to occur 44 seconds into the transient. The flow regime was not calculated this far into the transient.

The calculated flow regimes in the intact loop cold leg vessel inlet (Spool 15) for Tests S-01-1 B and S-01-4A are presented in Figure I-19. Significantly higher superficial phase velocities were calculated for Test S-014A than were calculated at this location for Test S-01-1B for the same time into the transient. The higher velocities for Test S-01-4A were due to the larger break flow existing between the break on the broken loop vessel inlet side and the intact loop vessel inlet in the $200 \%$ cold leg break configuration.

The horizontal and vertical densitometer measurements obtained during Test S-01-4A and S-01-1B at the intact loop cold leg vessel inlet are shown in Figures I-20 and I-21, respectively. For Test S-01-4A, the density measurements indicate dispersed bubble flow for the first 10 seconds of the transient, whereas the calculated flow regime is elongated bubble flow until about 13 seconds into the transient. Slug flow is calculated to exist from about 13 to 21 seconds, but the density measurements indicate stratified flow from about 10 to 21 seconds. Annular mist flow is not calculated to exist at all but is indicated by the densitometers from about 21 to 23 seconds. After the initiation of ECC injection, the densitometers indicate a period of what could be characterized as plug flow existing until 


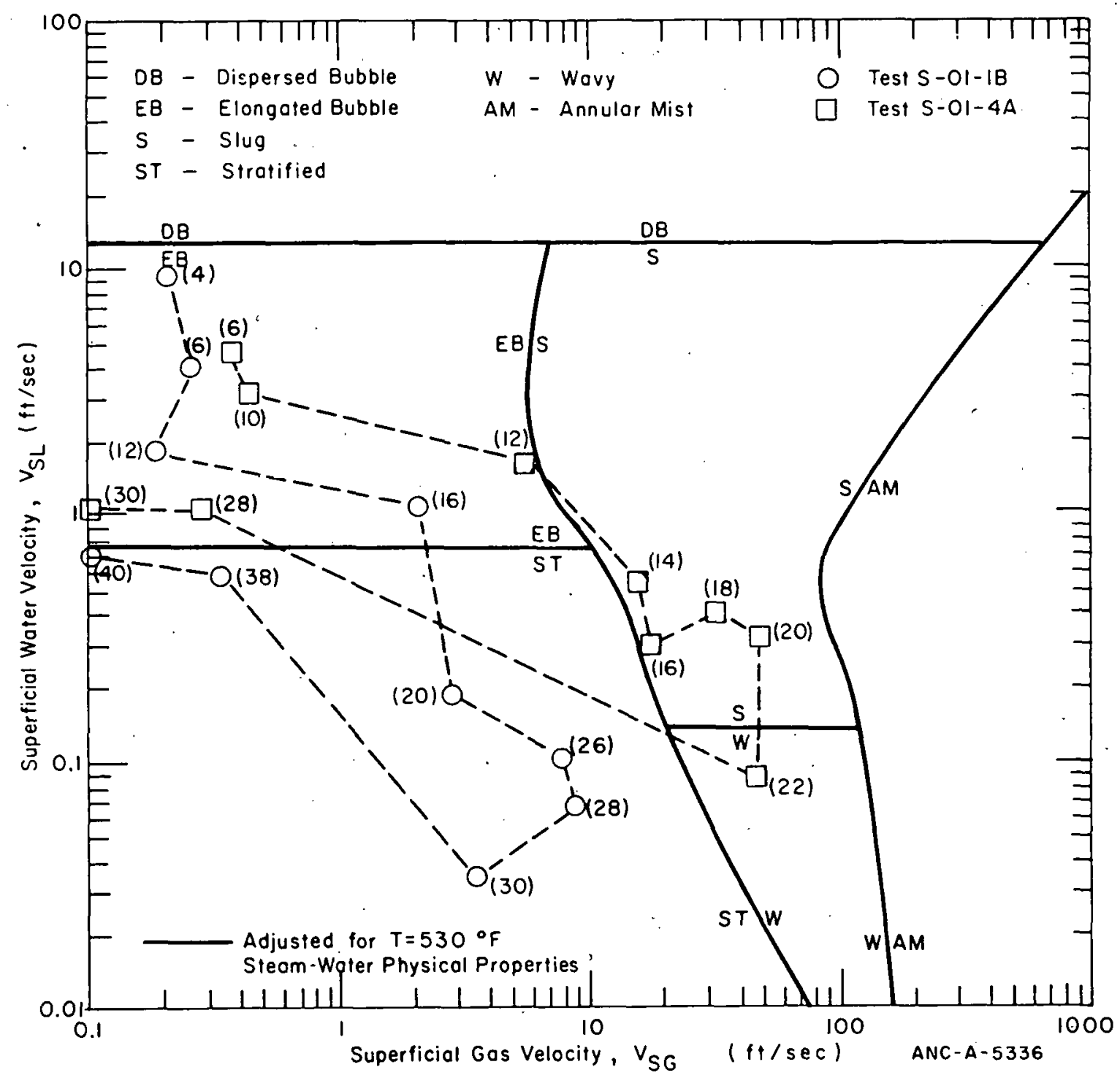

Fig. I-19 Flow regimes in the intact loop cold leg vessel inlet.

the piping is completely filled with injected coolant. Plug flow is the alternate formation and expulsion of a liquid plug in the intact loop cold leg.

The density information obtained at the intact loop cold leg vessel inlet during Test S-01-1B indicates dispersed bubble flow for the first 9 seconds of the transient, whereas the calculated flow regime was elongated bubble flow for the initial 18 seconds of the transient. The densitometers do indicate elongated bubble flow from about 9 to 15 seconds. The densitometers then, at first glance, appear to indicate a period of slug and elongated bubble flow. An examination of the flow rate measurements at this location, however, indicates very low mass flow rates over this time frame, substantiating the assumption that a stratified flow regime with varying vertical position of the liquid-steam interface is operative and remains so until about 37 seconds into the transient. The calculated flow regimes and densitometer data are in good agreement over this time period. 


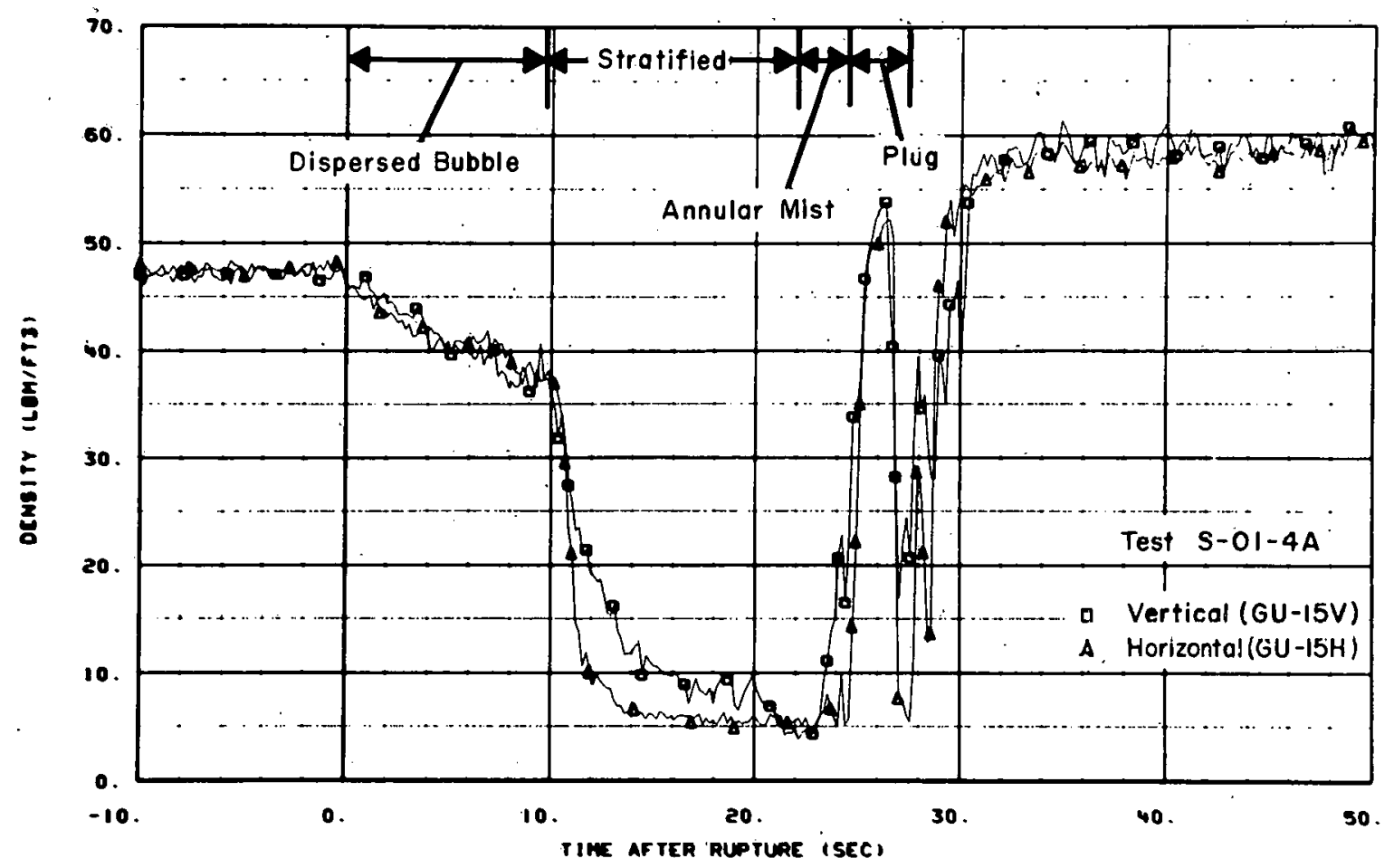

Fig. I-20 Flow regimes shown by the vertical and horizontal density measurements at the intact loop cold leg vessel inlet Test S-01-4A.

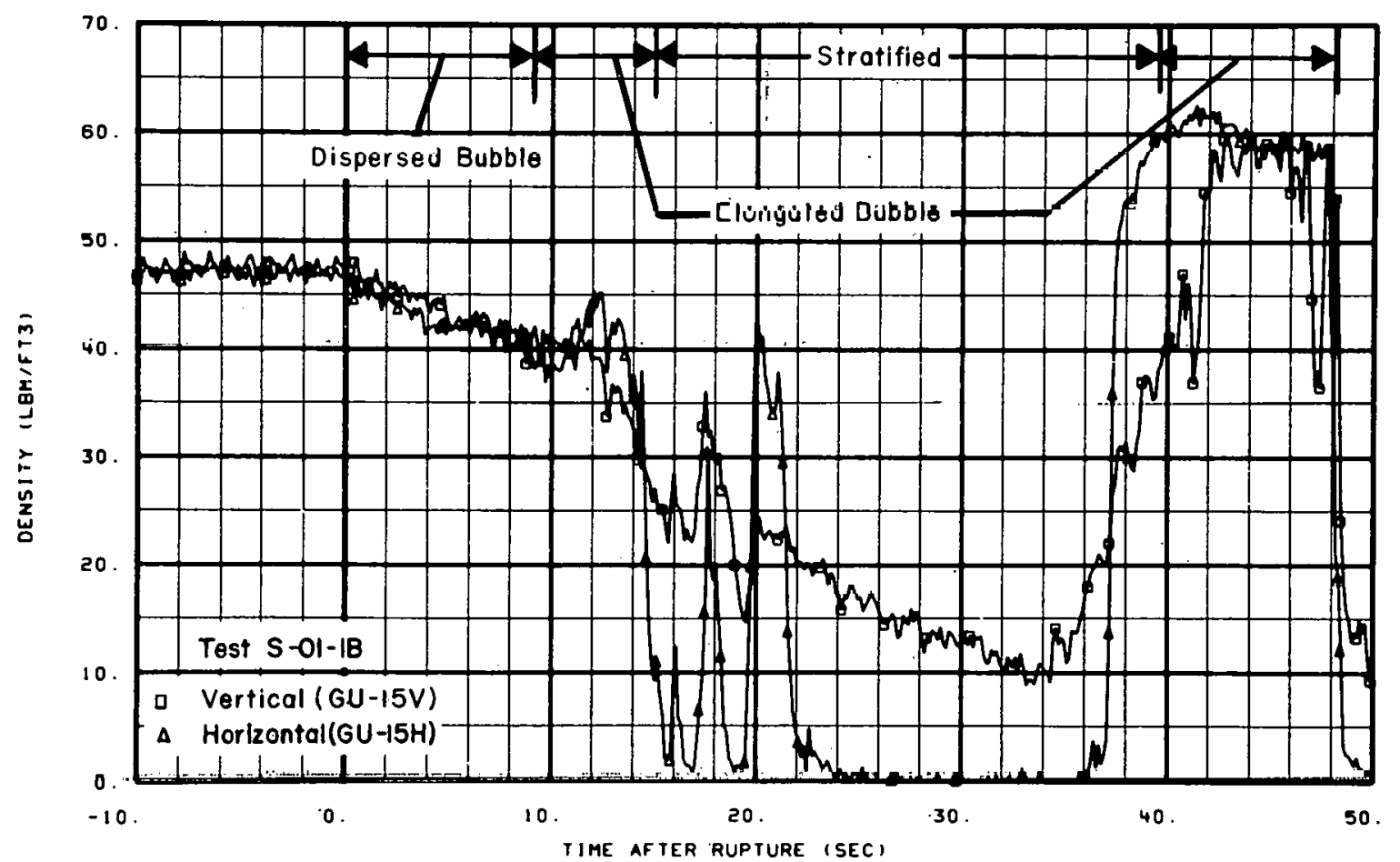

Fig. I-21 Flow regimes shown by the vertical and horizontal density measurements at the intact loop cold leg vessel inlet Test S-01-1'B. 
ECC injection was initiated during Test S-01-4A about 22 seconds into the transient. The injection point is in Spool 14 immediately upstream of the intact loop cold leg vessel inlet. The Test S-01-4A calculated flow regime information shows that the inlet has been completely filled with coolant 8 seconds after ECC injection initiation. ECC injection was initiated about 33 seconds into the transient during Test S-01-1B. The inlet, according to the flow regime calculations, is completely filled with coolant about 7 seconds after injection initiation. These inlet fill times agree well with the density measurements at this location.

The flow regime predictive method used in this report and the flow map of Mandhane et al appear to have reasonable utility in transient thermal-hydraulic cases. The densitometer data indicate an initial period of dispersed bubble flow, whereas the calculations indicate elongated bubble flow. The elongated bubble flow regime indicated by the data is, in general, predicted accurately. The predictions of slug flow were not borne out by the densitometer data. The calculations indicating stratified flow regimes (stratified and wavy) were in good agreement with the densitometer data. The calculated flow regimes for the $100 \%$ hot leg break case (Test S-01-1B) were, in general, more representative of the densitometer measurements than those for the $200 \%$ cold leg break configuration (Test S-01-4A). These results imply that the accuracy of the flow map of Mandane et al improves as the speed of the transient (phase velocities) decreases. Possibly after sufficient Semiscale Mod-1 data have been accumulated, new flow regime boundaries may be postulated which will be more applicable to transient thermal-hydraulic systems or at least to the Semiscale Mod-1 system.

\title{
3. INTACT LOOP PUMP PERFORMANCE
}

\author{
G. G. Loomis
}

In this section, the performance of the intact loop pump during the Semiscale Mod-1 isothermal blowdown tests is compared with the pump performance measured during previous pump tests and with the results obtained from analytical pump models based on these tests. The previous pump tests include the steady state single- and two-phase tests preformed at the Westinghouse Canada Limited (WC.L) facilities in Hamilton, Ontario, Canada $^{[I-13]}$, and the transient 1-1/2-loop isothermal blowdown tests ${ }^{[1-14]}$. performed in the Semiscale system at the Idaho National Engineering Laboratory (INEL). These comparisons provide insight into (a) the applicability of pump characteristics determined from steady state single-phase tests for describing the steady state operating point during the Semiscale Mod-1 isothermal tests, (b) the applicability of pump characteristics determined from steady state two-phase tests for describing the transient performance of the pump during the Mod-1 isothermal tests, and (c) the applicability of head degradation multipliers derived from the Semiscale 1-1/2-loop isothermal blowdown tests coupled with the pump characteristics obtained from the steady state single- and two-phase tests for analytically describing the head degradation during the Mod-1 isothermal tests. 


\subsection{Applicability of Steady State Single-Phase Test Data to Describe Mod-1 Pump Performance}

A single-phase pump characteristic curve derived from the steady state single-phase pump tests is shown in Figure I-22 along with the initial operating point for Mod-1 isothermal blowdown Test S-01-4A. For analytical purposes, the single-phase pump characteristic curve is plotted as a homologous pump curve represented by ratios of head, speed, and flow all normalized to the rated conditions. This single-phase homologous head curve was derived using a statistical fit to scattered steady state pump data. Comparison of the initial operating point of the Mod-1 pump during isothermal blowdown tests with the single-phase homologous curve shows that the initial operating point is slightly high for Test S-01-4A. In fact, between values of normalized speed/normalized flow $(\alpha / v)$ of 0.45 and 1.0 , the homologous head curve in the normal opcrating quadrarit would better represent the Mod-1 isothermal operating point if it were approximated by a straight line defined by

$$
\frac{\mathrm{h}}{\mathrm{v}^{2}}=1.85 \frac{\alpha}{\mathrm{v}}-0.83
$$

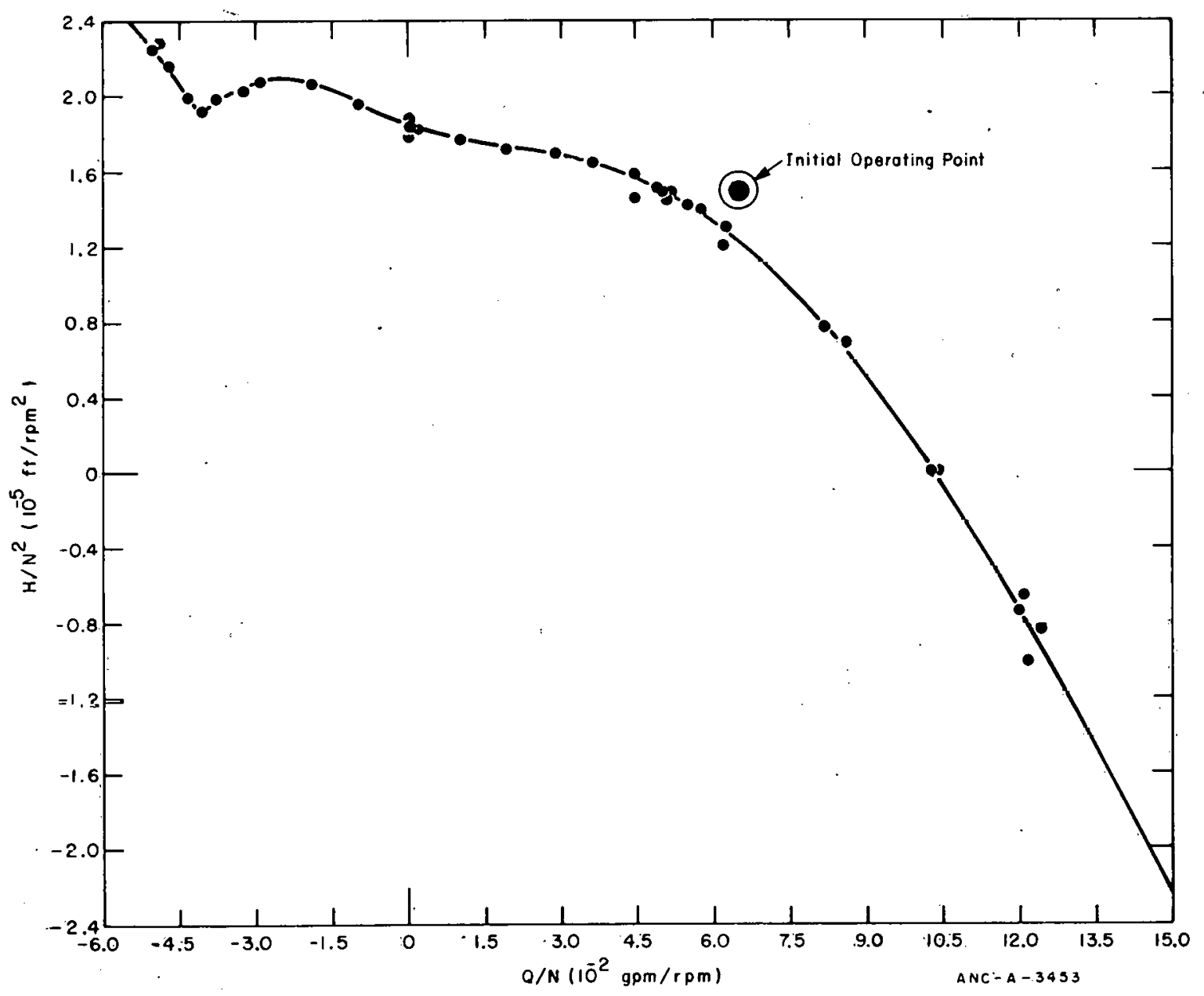

Fig. I-22 Semiscale Mod-1 pump characteristic curve with initial operating point for Test S-01-4A. 


\subsection{Applicability of the Steady State Two-Phase Tests to Describe Mod-1 Pump Performance}

Previous analysis of the data taken during steady state two-phase tests ${ }^{[\mathrm{I}-15]}$ on the Mod-1 intact loop pump indicated that these data were inadequate for plotting definite curves of head as a function of void fraction. Instead, bands of operation over different ranges of void fraction were defined for curves of head divided by speed squared $\left(\mathrm{H} / \mathrm{N}^{2}\right)$ versus pump flow divided by speed $(\mathrm{Q} / \mathrm{N})$. These bands of operation were then compared with transient pump performance data obtained during the Semiscale 1-1/2-loop isothermal test series, and good agreement between the steady state and transient results was noted.

However, calculations of void fraction, $\mathrm{Q} / \mathrm{N}$, and $\mathrm{H} / \mathrm{N}^{2}$. using the transient Semiscale Mod-I isothermal pump data show that the agreement between the steady state results and the transient Mod-1 data is poor over a wide range of inlet void fractions. The inlet void fraction for the Mod-1 tests was estimated in the same manner used to define the bands of operation for various void fractions in Reference I-15. This method involved establishing an effective density at the pump inlet which was corrected for flow regime. Use of an effective density was necessary to estimate the void fraction because, as indicated in Reference I-4, separated flow regimes existed at the pump inlet for the transient blow down. Reference I-4 also indicated that for $200 \%$ cold leg break tests, the flow regime varied from elongated bubble to slug flow for the first 15-20 seconds. Both of these regimes are, in a sense, stratified; therefore, the flow was assumed stratified for the calculation of inlet void fraction. Reference I-4 indicated that after 20 seconds, the flow regime was stratificd.

Comparison of the previous steady state two-phase test results with data from Mod-1 isothermal Test S-01-3 are presented in Figures I-23 through I-25 on normalized head curves for three separate time intervals: 0 to 10 seconds (Figure I-23), 10 to 20 seconds (Figure I-24), and 20 to 30 seconds (Figure I-25). Two numbers are in parentheses by each normalized data point for Test S-01-3. The first number refers to the time after rupture in seconds, and the second number is the pump inlet void fraction in percent. The Mod-1 isothermal results presented in Figure I-23 show the pump head remained positive for the first 10 seconds of the transient. The effect of the initial flow surge which was caused by acceleration of fluid through the pump is shown as the data starts at the 0 time point and moves toward higher $\mathrm{Q} / \mathrm{N}$. As the pump inlet flow rate then decelerates and decreases, the trace swings back toward smaller $\mathrm{Q} / \mathrm{N}$. For the first 4 seconds, the Mod-1 normalized data show good agreement with the band of void fraction developed from the steady state tests corresponding to $\alpha \leqslant 18 \%$. After 4 seconds, the corresponding void fractions of the Mod-1 isothermal data points are lower than the void fractions corresponding to the bands generated from the steady state tests. For instance, at 5 seconds the void fraction for the Mod-1 data is $10 \%$, but the data point lies in a steady state void fraction region of 14 to $30 \%$. This trend of smaller Mod-1 void fraction data points falling into the steady state bands of higher void fraction for times greater than 4 seconds indicates that the pump head was degraded more, at a given void fraction, for the Mod-1 isothermal tests than for the steady state test. At about -13 seconds the pump head becomes fully degraded and remains in this state until about 24 seconds (Figures I-24 and I-25). The Test S-01-3 normalized points are again consistently lower than the bands calculated from the steady state data. At 


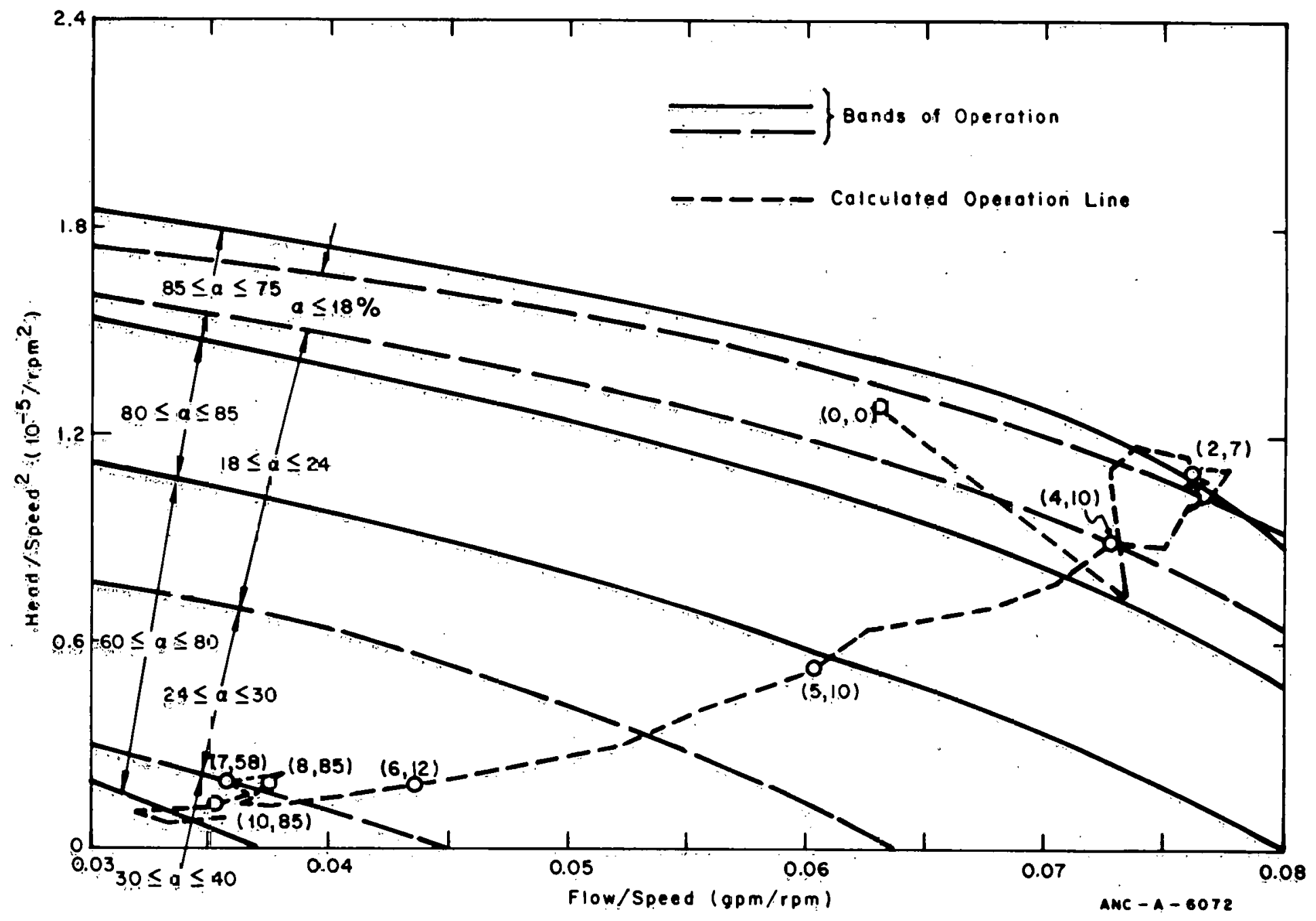

Fig. I-23 Normalized head versus :Tow for various inlet void fractions from 0 to 1 J seconds - Test S-01-3. 


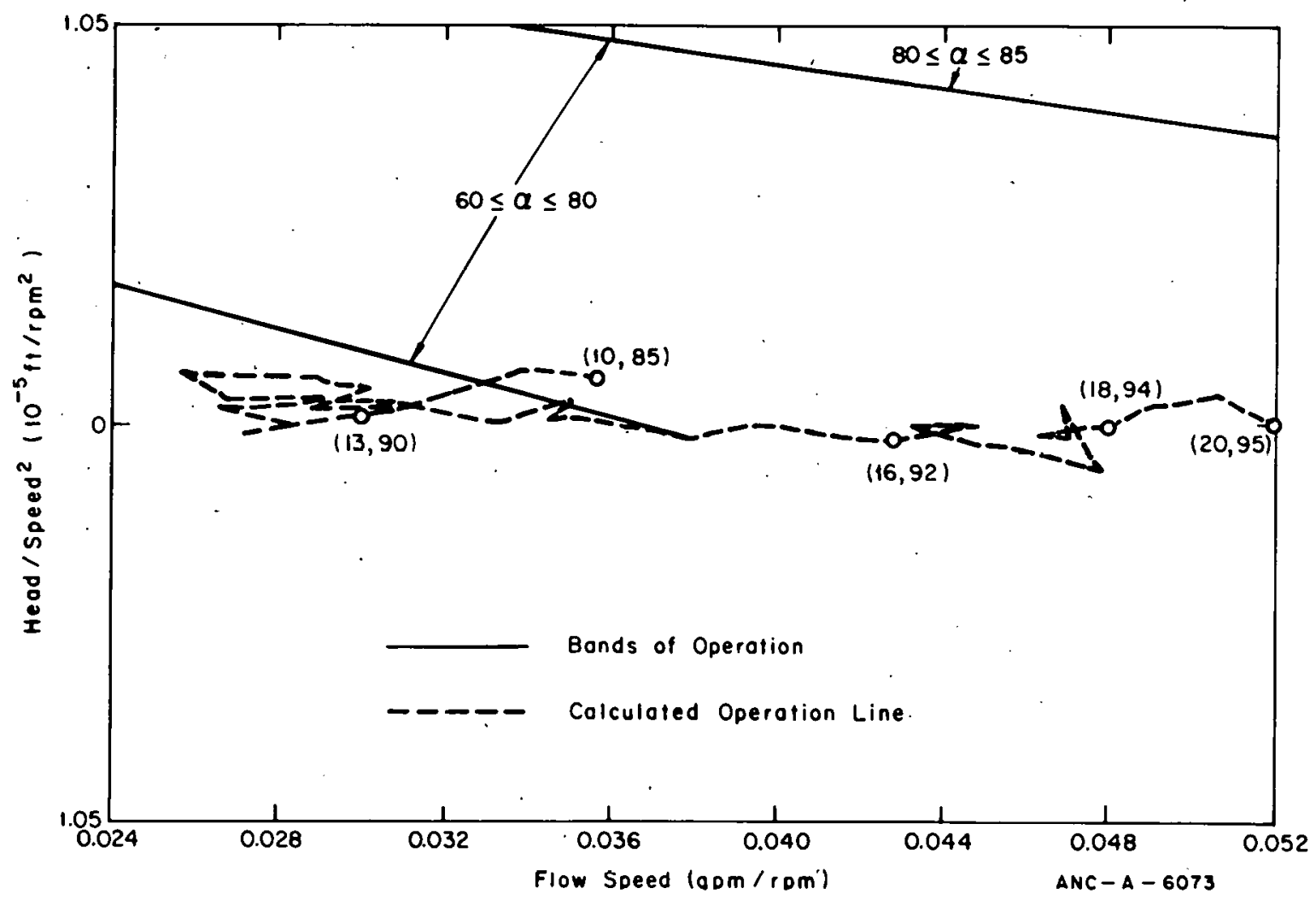

Fig. I-24 Normalized head versus flow for various inlet void fractions from 10 to 20 seconds - Test S-01-3.

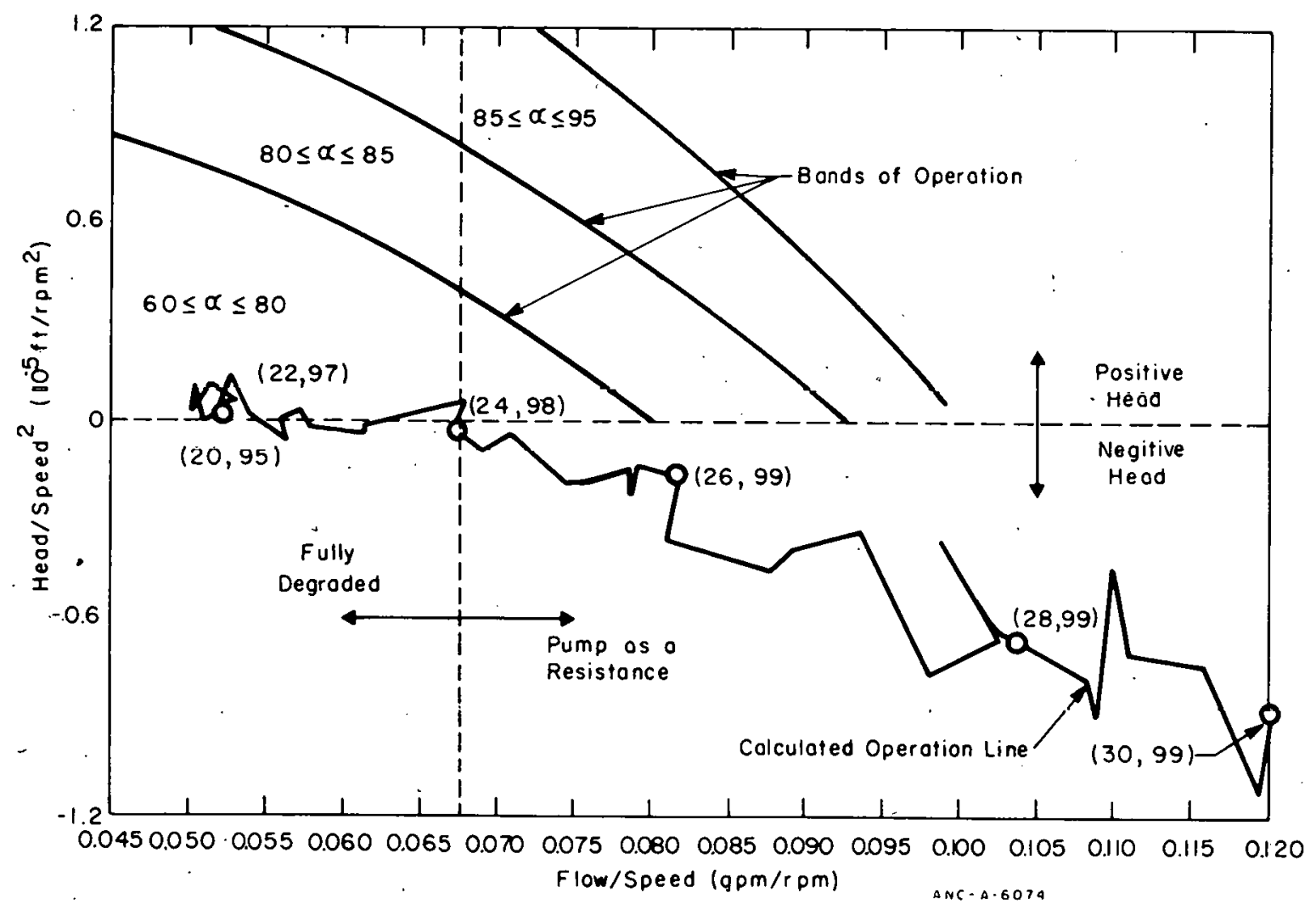

Fig. I-25 Normalized head versus flow for various inlet void fractions from 20 to 30 seconds - Test S-01-3. 
about 24 seconds the $\mathrm{H} / \mathrm{N}^{2}$ becomes negative, indicating that the pump has become a resistance to flow.

3.3 Applicability of Steady State Single- and Two-Phase Tests and Transient 1-1/2-Loop Tests for Analy tically Describing Mod-1 Pump Performance

The existing pump model contained in the RELAP4 ${ }^{[I-6]}$ computer code is based on experimental data taken during the steady state single- and two-phase tests and also during the Semiscale 1-1/2-loop transient isothermal tests. Data from the steady state single- and two-phase tests were used to develop single- and two-phase homologous head curves, and the data from the transient 1-1/2-loop isothermal tests were used along with the homologous head curves to develop the head degradation multiplier curve.

Since the existing RELAP4 pump model ${ }^{[I-6]}$ is based on the steady state single- and two-phase test data which have heen shown to disagree somewhat with Mod-1 normalized test data, calculations of Mod-1 transient performance using the RELAP4 model would be expected to be inaccurate. Several calculations were made independent of the complete RELAP4 computer code, using Test S-01-4A data and the equations and logic from the RELAP4 pump model to assess the effect of using the steady state basis for Mod-1 calculations. Pump inlet and outlet fluid densities, pump flow, and pump speed were taken from Semiscale Mod-1 data and converted to parameters compatible with the pump model. The calculated parameters were average void fraction and the homologous ratio of (flow/rated flow)/(speed/rated speed) and the inverse of this quantity. These normalized parameters, the homologous single- and two-phase difference pump curves, and the pump two-phase multiplier curve contained in the RELAP4 pump model were used to determine the ratios of (pump head/pump rated head)/(flow/rated flow) ${ }^{2}$ or (pump head/pump rated head)/(pump speed/pump rated speed $)^{2}$. By following the equations outlined in Reference I-6 for calculating pump head from the homologous curves, the pump differential pressure was calculated for Mod-1 Test S-01-4A. Two different two-phase head multipliers were used in the independent calculations. One multiplier, $\mathrm{M}_{2}(\alpha)$, was based on the steady state two-phase pump test results $[\mathrm{I}-13]$ and the other multiplier, $\mathrm{M}_{3}(\alpha)$, was formulated from the transient data of the Semiscale 1-1/2-loop isothermal blowdown series tests ${ }^{[\mathrm{I}-14]}$ and was the degradation multiplier used in all of the RELAP4 calculations for the Mod-1 isothermal test'system behavior. These two multipliers, shown in Figure I-26, are significantly different in the void fraction regions from 0.08 to $\cdot 0.3$. Results from the independent calculations using the two different two-phase multipliers $\mathrm{M}_{2}(\alpha)$ and $\mathrm{M}_{3}(\alpha)$ along with Test S-014A data and system calculations using the RELAP4 code for Test S-01-4A are presented in Figure I-27. For the first 2 seconds, the independent calculations using $\mathrm{M}_{2}(\alpha)$ and $\mathrm{M}_{3}(\alpha)$ are both slightly lower than the data, indicating that the single-phase homologous head curves may be slightly low for the speeds and flows encountered during this time period. The inlet void fraction based on the Test S-01-4A data is sufficiently low for the first 3 seconds, and essentially no two-phase pump head degradation occurs as indicated by the agreement of the $M_{2}(\alpha)$ and $M_{3}(\alpha)$ calculations. After 3 seconds, the data show considerable pump head degradation; but both independent calculations and the RELAP4 calculation are consistently higher than the data. Both independent calculations exhibit a rise in differential pressure between 3 and 4 seconds 


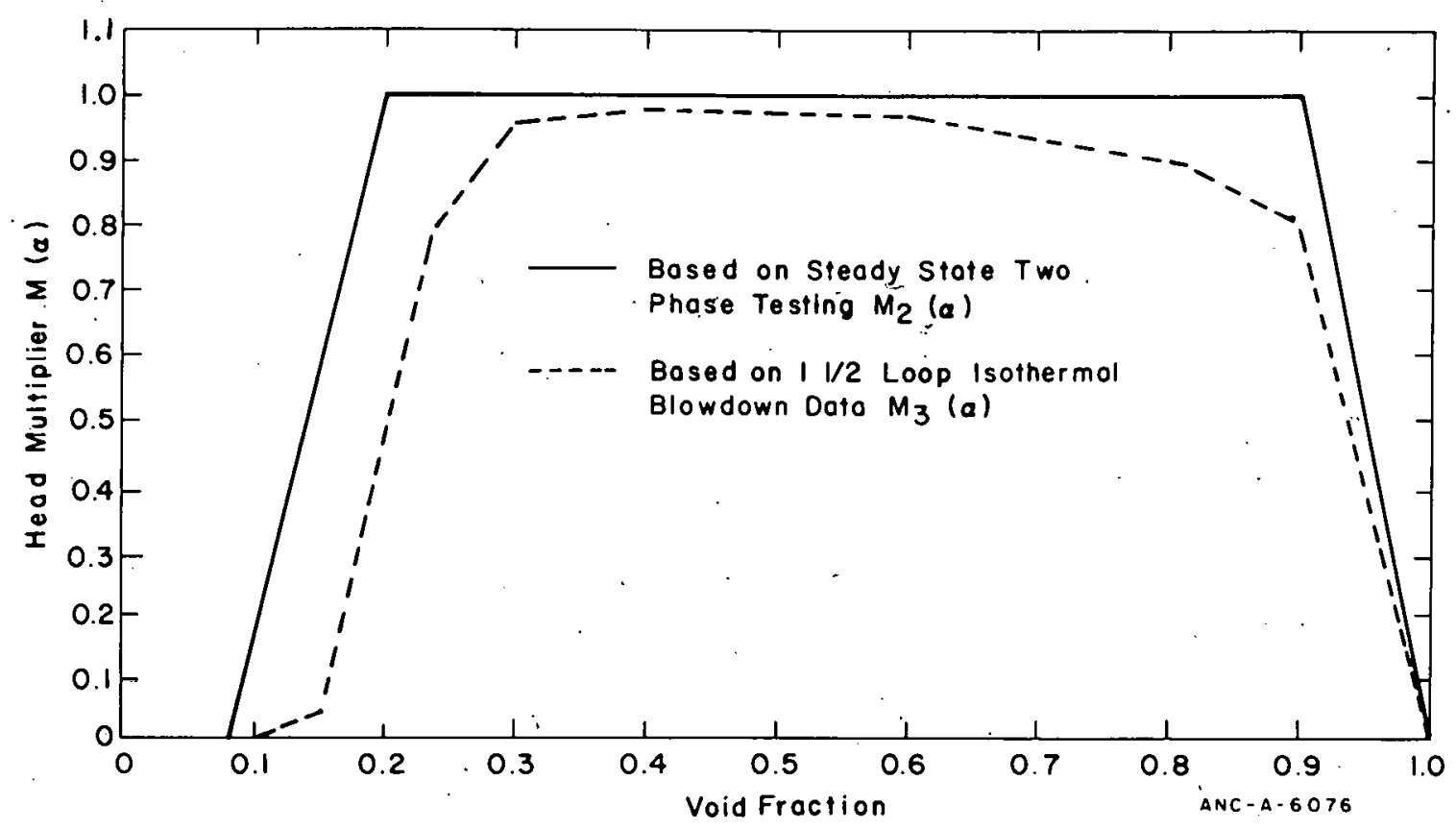

Fig. I-26 Two-phase head multipliers.

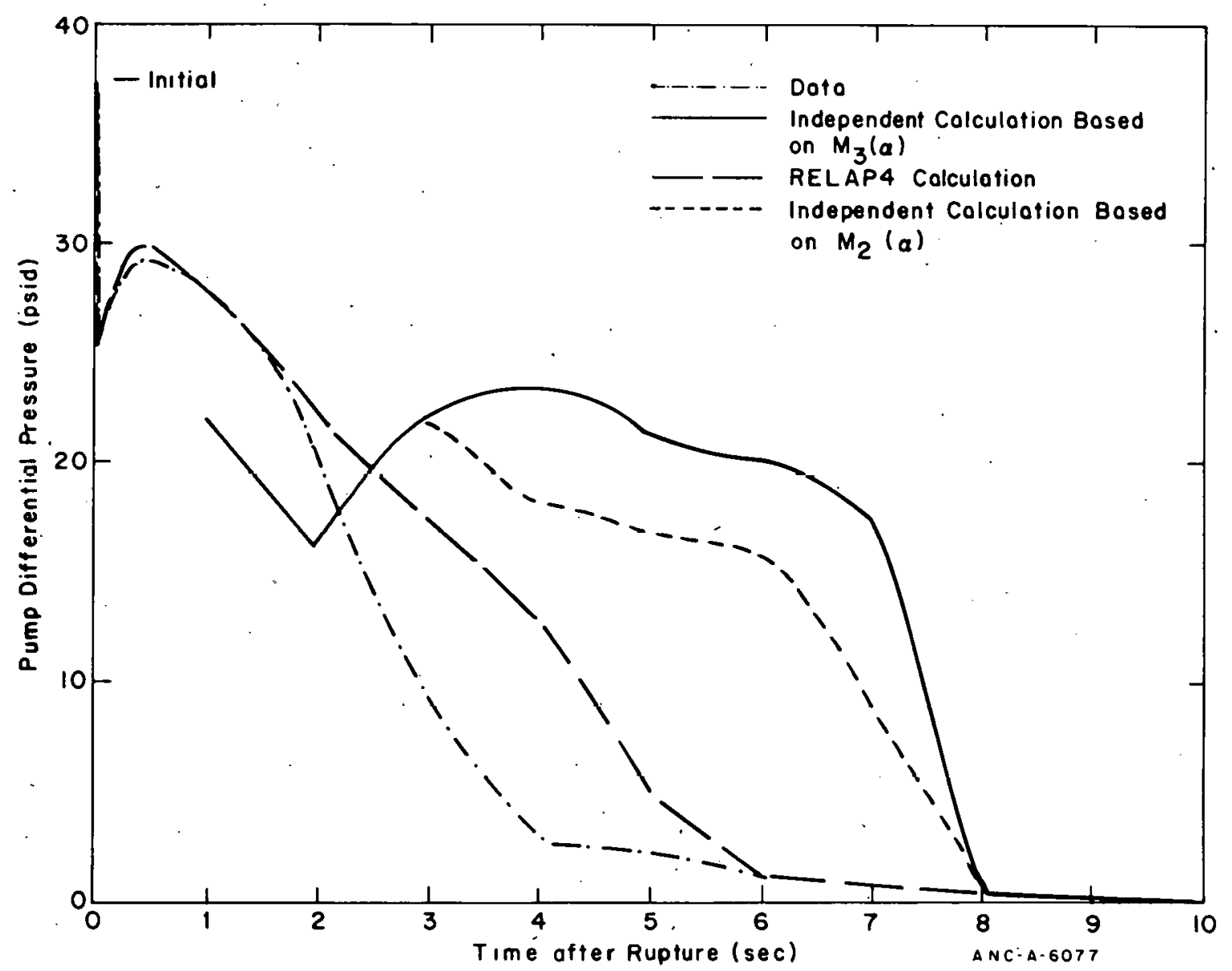

Fig. 1-27 l'est $S-01-4$ A pump ditferential pressure calculated using RELAP4 pump model. 
which is a trend significantly different than shown by the data and indicates insufficient degradation of the differential pressure during this time period. The differential pressure calculation from the RELAP4 system analysis during the period 3 to 8 seconds follows the data more closely than the independent calculations because of an underprediction of inlet density which resulted in a higher two-phase head degradation multiplier. If the RELAP4 system calculation, which used $\mathrm{M}_{3}(\alpha)$, had calculated the pump inlet and outlet densities, pump speed, and pump inlet flow correctly, the pump differential pressure would have followed the differential pressure curve independently using $\mathrm{M}_{3}(\alpha)$.

The fact that the calculated pump differential pressure using test data and the RELAP4 pump model does not agree well with the measured pump differential pressure appears to be the result of a slightly higher operating point for the Mod-1 pump than would be indicated by the pump homologous curve plus more head degradation than indicated by the head degradation multipliers.

\subsection{Development of a New Head Degradation Multiplier for Use in the RELAP4 Model}

The scatter in the experimental data used to develop the $\mathrm{M}_{2}(\alpha)$ and $\mathrm{M}_{3}(\alpha)$ multiplier curves discussed in Section I-3.3 resulted in a large uncertainty in the region of 0 to $30 \%$ inlet void fraction. Since use of $M_{2}(\alpha)$ and $M_{3}(\alpha)$ in the RELAP4 Mod-1 pump model resulted in a pump differential pressure that is appreciably different than the Mod-1 isothermal test data, a third multiplier based on results from Mod-1 isothermal tests was developed. This new two-phase degradation multiplier curve was derived from the calculated head degradation multiplier versus void fraction points which were obtained utilizing the RELAP4 pump model and Mod-l isothermal test data. Data from three tests, Tests S-01-1B $[\mathrm{I-14}]$, S-0l-4A ${ }^{[\mathrm{I}-15]}$, and S-01-5[I-16] , were used. The RELAP4 homologous single-phase curve for normal pump operation (HVN) was modified as discussed previously for this calculation [Equation (I-1) in Section I-3.1] so that the initial single-phase operation point for Mod-l tests was directly on the homologous HVN curve.

The pump head degradation multipliers $\mathrm{M}_{2}(\alpha)$ and $\mathrm{M}_{3}(\alpha)$ are plotted on Figure I-28 along with an estimated best fit to the Mod-1 isothermal degradation points labeled $\mathrm{M}_{\mathrm{m}}(\alpha)$. As expected, $\mathrm{M}_{\mathrm{m}}(\alpha)$ is consistently higher than both $\mathrm{M}_{2}(\alpha)$ and $\mathrm{M}_{3}(\alpha)$ in the 0 to $30 \%$ void fraction region which will result in a calculated differential pressure which is lower. Figure $\mathrm{I}-29$ is a plot showing l'est $\mathrm{S}-\mathrm{OI}-4 \mathrm{~A}$ pump differential pressure data and the calculated pump differential pressure obtained through use of the equations from the RELAP4 model, the modified single-phase homologous curve, $M_{2}(\alpha), M_{3}(\alpha)$, and $M_{m}(\alpha)$. As expected, use of $\mathrm{M}_{\mathrm{m}}(\alpha)$ results in better agreement with the data than does use of the multipliers $M_{2}(\alpha)$ and $M_{3}(\alpha)$ for the Mod-1 tests. Calculations for other tests in the isothermal test series show similar results.

Since the instrumentation used for measuring the pump performance was better for the Mod-1 isothermal test series than for the previously conducted Semiscale 1-1/2-loop isothermal or steady state tests, $M_{m}(\alpha)$ would be expected to be more nearly accurate than $\mathrm{M}_{2}(\alpha)$ or $\mathrm{M}_{3}(\alpha)$ and would, therefore, be expected to provide better RELAP4 calculations 


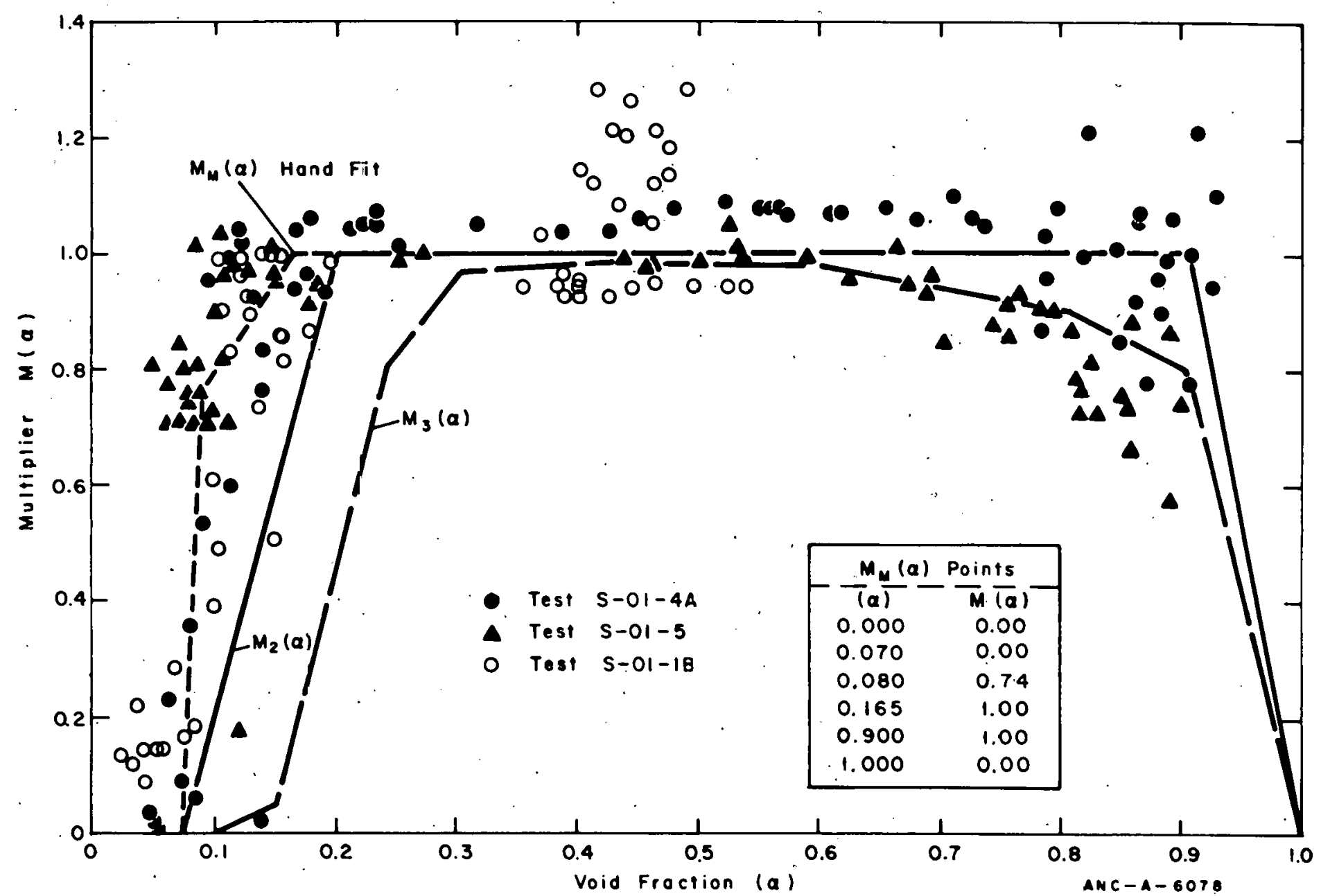

Fig. I-28 Three two-phase multipliers for the Semiscale Mod-1 pump. 


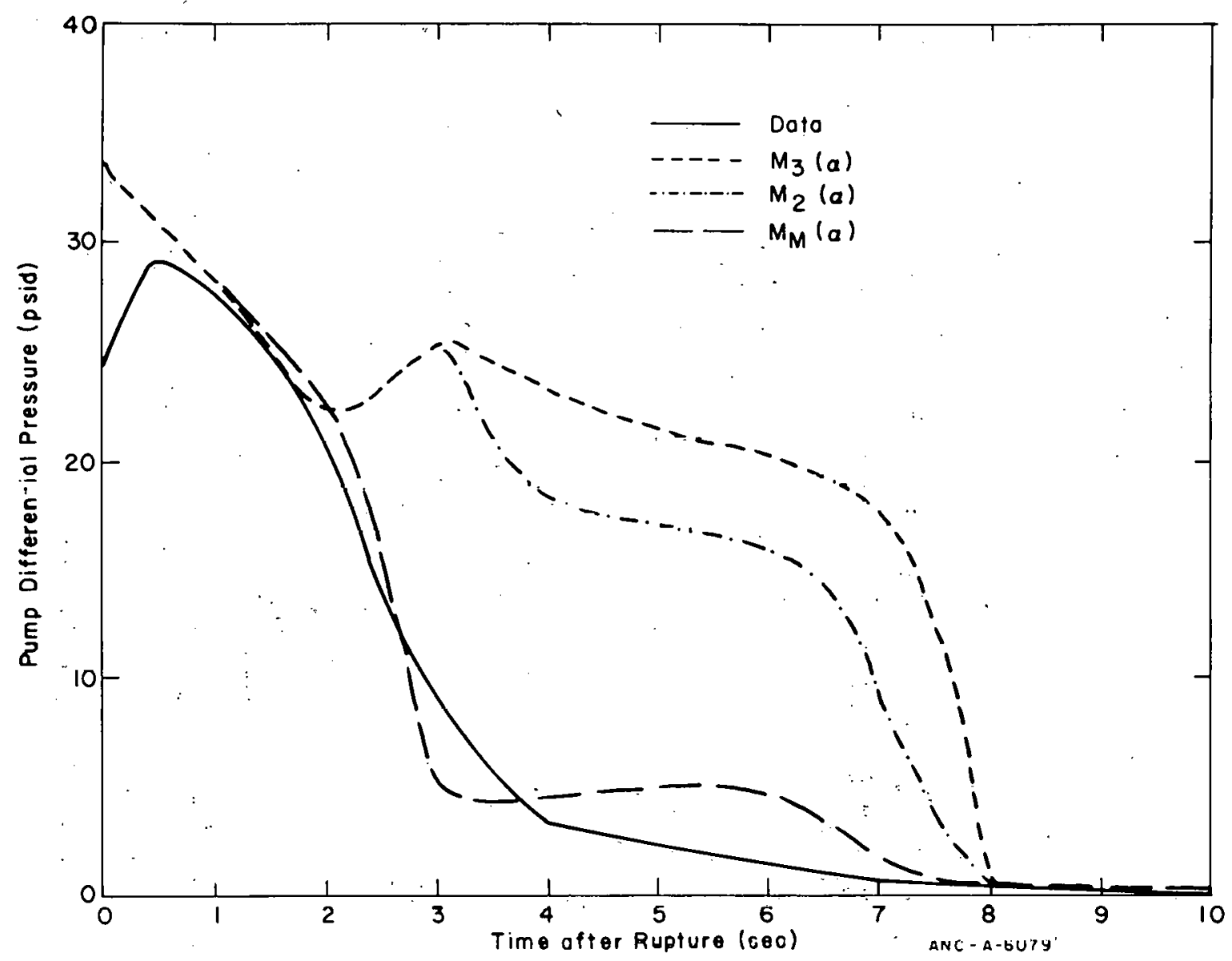

Fig. I-29 Pump differential pressure calculated using various multipliers.

of pump differential pressure for future Mod-1 tests. Further tests in the Mod-1 program are expected to provide data that can also be used to refine the $\mathrm{M}_{\mathrm{m}}(\alpha)$ multiplier. Significant improvement in the two-phase multipliers is expected when instrumentation capable of distinguishing flow regimes is installed at the pump suction later in the Mod-1 program.

\section{REFERFNCFS}

I-1. Aerojet Nuclear Company, Quarterly Technical Report on Water Reactor Safety Programs Sponsored by the Nuclear Regulatory Commission's Division of Reactor Safety Research, January - March 1975, ANCR-1254 (September 1975).

I-2. D. J. Hanson, R. T. French, C. J. Shaffer, An Evaluation of ECC Related Phenomena. During Semiscale Mod-1 Isothermal Tests, ANCR-1239 (November 1975).

I-3. C. E. Cartmill, Thermal-Hydraulic Response of the Semiscale Mod-1 System Isothermal Test Series, ANCR-1228 (October 1975). 
I-4. R. T. French, An Evaluation of Piping Heat Transfer, Piping Flow Regimes, and Steam Generator Heat Transfer for the Semiscale Mod-1' Isothermal Tests, ANCR-1229 (August 1975).

I-5. G. G. Loomis, Intact Loop Pump Performance During the Semiscale Mod-1 Isothermal Test Series, ANCR-1240 (October 1975).

I-6. K. V. Moore and W. H. Rettig, RELAP4 - A Computer Program for Transient Thermal-Hydraulic Analysis, ANCR-1 127 (December 1973).

I-7. R. E. Henry and H. K. Fauske, "The Two-Phase Critical Flow of One-Component Mixtures in Nozzles, Orifice, and Short Tubes", Journ. of Heat Trans., Trans. ASME (May 1971).

I-8. F. J. Moody, "Maximum Flow Rate of a Single Component, Two-Phase Mixture"; Journ. of Heat Trans., Trans. ASME (February 1965).

I-9. G. W. Govier and J. Aziz, The Flow of Complex Mixtures in Pipes, Great Britain: Van Nostrand Reinhold Company, 1972.

I-10. J. M. Mandhane, G. A. Gregory, K. Aziz, "A Flow Pattern Map for Gas-Liquid Flow in Horizontal Pipes", Int. J. Multiphase Flow, Vol. I, Pergamon Press, 1974.

I-11. O. Baker, Oil and Gas Journal, 53 (1954) p 185.

I-12. C. J. Hoogendoorn, Chem. Eng. Sci., 9 (1959) p 205.

I-13. D. J. Olson, Experiment Data Report for Single-and Two-Phase Steady State Tests of the 1-1/2-Loop Semiscale System Pump, ANCR-1 150 (May 1974).

I-14. H. S. Crapo, M. F. Jensen, K. E. Sackett, S. N. Zender, Experiment Data Report for Semiscale Mod-1 Tests S-01-1B (Isothermal Blowdown with Core Resistance Simulator), ANCR-1199 (May 1975).

I-15. S. N. Zender, M. F. Jensen, K. E. Sackett, Experiment Data Report for Semiscale Mod-1 Tests S-01-4 and S-01-4A (Isothermal Blowdown with Core Resistance Simulator), ANCR-1196 (March 1975).

I-16. S. N. Zender, H. S. Crapo, M. F. Jensen, K. E. Sackett, Experiment Data Report for Semiscale Mod-1 Test S-01-5 (Isothermal Blowdown with Core Resistance Simulator), ANCR-1197 (April 1975). 


\title{
II. LOFT:PROGRAM
}

\section{J. Ybarrondo, Program Manager}

This section of the report contains discussions of an uncertainty analysis performed on the LOFT experimental measurements system and of the hydraulic parameters determined for the primary coolant system during acceptance testing of the LOFT Integral Test System.

A description of the LOFT Integral Test System and a discussion of its major program objectives are presented in Reference II-1.

\section{LOFT EXPERIMENTAL-MEASUREMENTS UNCERTAINTY ANALYSIS}

\author{
G. L. Biladeau, L. D. Goodrich, P. A. Quinn
}

To establish the validity of the loss-of-coolant experiments (LOCE) to be performed by the LOFT Integral. Test System, the performance of the test instrumentation.and data acquisition system must first be identified. The LOFT Experimental Measurements Uncertainty Analysis is an evaluation of the anticipated performance uncertainty for each experimental measurement to be made in the LOFT Program and is based on analysis of test results to date, calculations, standards, and/or manufacturer's specifications. This report summarizes the analy sis results.

\subsection{General Description of LOFT Experimental Measurement System}

The LOFT Program requires a large volume of measurements (about 200 for a nonnuclear test and about 600 for a nuclear test) of a diverse nature, which present a multitude of circuit and system considerations. These measurements require a system of considerable sophistication to condition, calibrate, acquire, record, convert, and process measurement signals with sufficient accuracy, precision, reliability, and speed to be of full use. As shown in Figure II-1, the LOFT Experimental Measurement System san conceptually be divided into three sections: (a) transducers, (b) data acquisition and visual display system (DAVDS), and (c) data processing, both on- and off-site. A brief discussion of these three sections follows:

1.1.1 Transducers. "A transducer is a device which provides a usable output in response to a specific measurand"[II-2]. In LOFT, the environment in which some of the transducers must perform is quite hostile (e.g., pressures to 2,500 psia, temperatures to $2,500^{\circ} \mathrm{F}$, thermal neutrons to $10^{14} \mathrm{n} / \mathrm{cm}^{2} \mathrm{sec}$, and gamma radiation to $10^{9} \mathrm{R} / \mathrm{hr}$, including severe transients in these parameters). Transducers from commercial manufacturers have not always been suitable for this application, necessitating state-of-the-art developments by Aerojet Nuclear Company. 


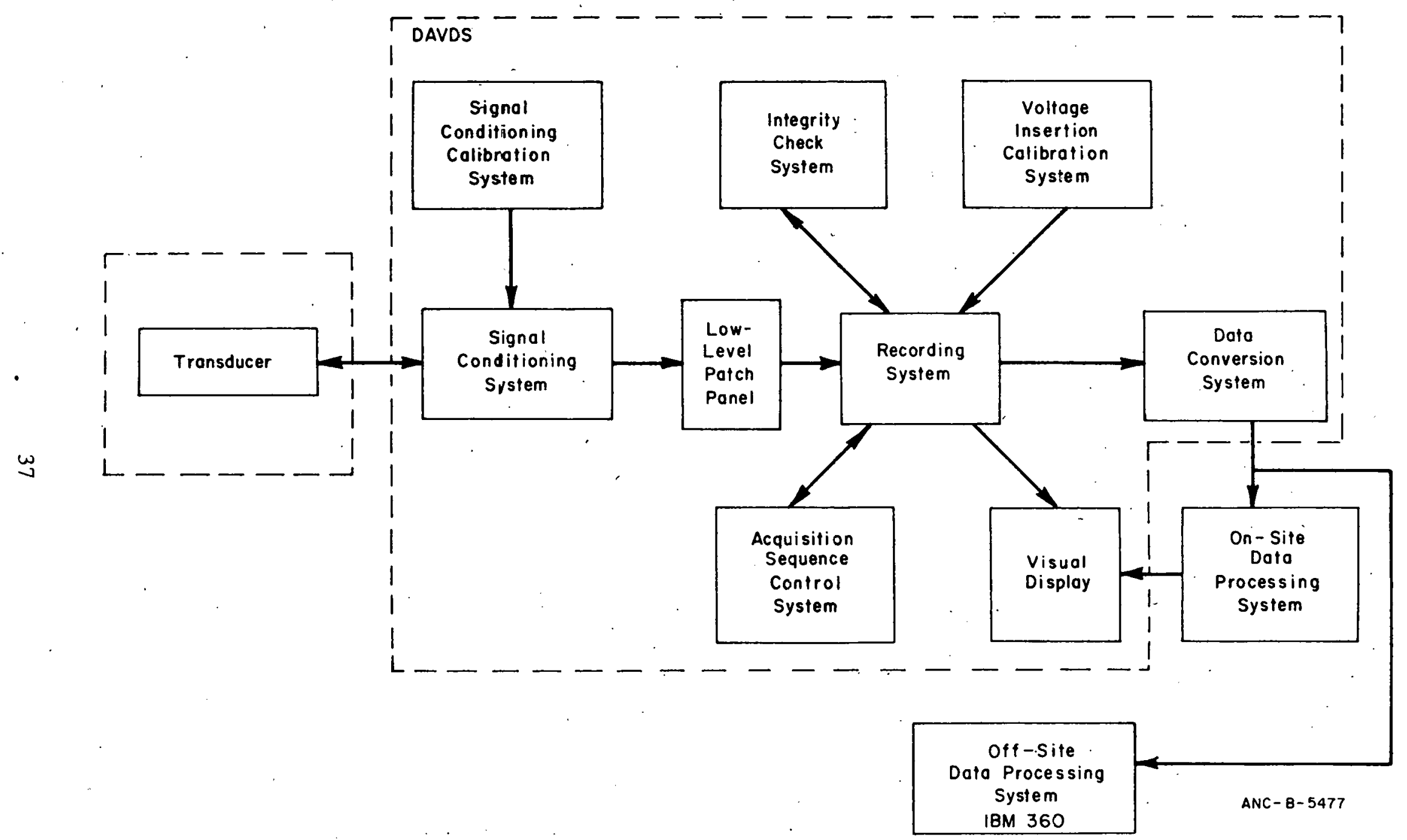

Fig, II-1 Block diagram of LOFT Experimental Measurement System. 


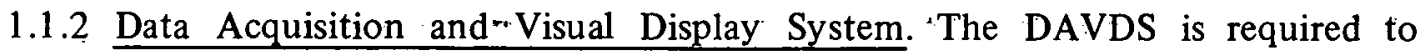
condition, record, and display experimental data. While the signal format (voltages, impedances) presented to the recording equipment must be uniform, the signals generated by the transducers arrive in a great many forms. Nearly every transducer type requires its own special conditioning electronics to ensure compatibility with the data recording equipment.

Though the signal format to the input of the recording channels is uniform, the bandwidths and intended use of these signals necessitates a variety of acquisition-record systems. Signal bandwidth requirements vary from $10 \mathrm{~Hz}$ to $20 \mathrm{kHz}$. Specific data usages include (a) control room display of both experimental and process parameters, (b) recording for posttest analysis, (c) on-line display of selected measured variables for test personnel, and (d) on-linc display of selected mieasured varlabbles for test observers from outside organizations

1.1.3 Digital Data Acquisition and Data Processing. A minicomputer, MODCOMP II, whlch is in addition to the IBM 360 located in the ANC Computer Science Centcr, performs the data processing for the LOFT Experimental Measurement System. The MODCOMP II is an integral part of the DAVDS in that it controls sequencing and calibration, performs status verification and system integrity checks, and acquires up to 546 channels of data.

From a data processing point of view, MODCOMP II may also do posttest digitizing of analog records (Neff 620 amplifier converts the analog signal to digital signal A/D for recording on magnetic tape) and perform the engineering units conversion.

\section{$1.2 \Lambda$ nalysis Rcsults}

The results of the measurement uncertainty analysis are compared to the measurement requirements ${ }^{[a]}$ in each of sixteen measurement categories.

A summary of the uncertainty analysis for each of the 16 measurement categories is presented in the following sections. The DAVDS (minus signal conditioners) and the calibration systems are presented first to allow total uncertainties for a given measurement to be subsequently determined in the applicable section. Uncertainty is given as a plus or minus $( \pm$ ) percent of the measurement range (RG) or percent of measurement reading (RD) having a $95 \%$ confidence value $(2 \sigma)$, where $\sigma$ is the standard deviation. Also, in the uncertainty analysis tables, the total uncertainty value is the root sum square (RSS) of the component values. Where sufficient data were not availahle for a romplete assessment of uncertainties at this time, it is so noted, and the uncertainty stated is the best estimate at this time.

[a] The measurement requirements are as established in the MRD-1, "LOFT Measurement Requirements Document" (February 28, 1975) and in the applicable system design descriptions (SDD). 
It must be noted that these values are calculations of composite uncertainty and response times utilizing specific sets of assumptions. Many of the individual uncertainty values are dependent on calibration and data reduction procedures or other factors. It should also be noted that these analyses do not include system noise which will be included when measured system noise levels become available.

1.2.1 DAVDS. All experimental measurement channel data must be conditioned, recorded, reduced, and displayed as experimental data. In LOFT, this is accomplished using the DAVDS.

The DAVDS consists of three distinct subsystems: (a) the wide-band frequency modulation (WBFM) system, (b) the multiplexed frequency modulation (MFM) system, and (c). the digital data acquisition and processing system (DDAPS). The accuracy design goals of the three systems were 1.0 percent of range $(1.0 \mathrm{RG})$ for the WBFM system, $1.0 \mathrm{RG}$ for the MFM system, and $0.25 \mathrm{RG}$ for the DDAPS system. These goals have essentially been met with calculated performance of $1.1 \mathrm{RG}, 0.96 \mathrm{RG}$, and $0.13 \mathrm{RG}$, respectively. The final decision as to which measurement channels are assigned to which DAVDS recording channels has not been made. However, total uncertainty values shown, in the summary are based on the intended recording channel. The volume, as well as the required bandwidth, is an additional consideration in establishing channel assignments. The channels available are as follows: 54 channels at $20 \mathrm{kHz}$. for the WBFM system, 100 channels at $1 \mathrm{kHz}$ for the MFM system, and 546 channels at $10 \mathrm{~Hz}$ for the DDAPS system. Tables II-I through II-III summarize the DAVDS uncertainty analysis. Preliminary analysis of data from the system operational acceptance test indicates observed accuracies are within the calculated accuracies.

TABLE II-I

SUMMARY OF WBFM UNCERTAINTY ANALYSIS

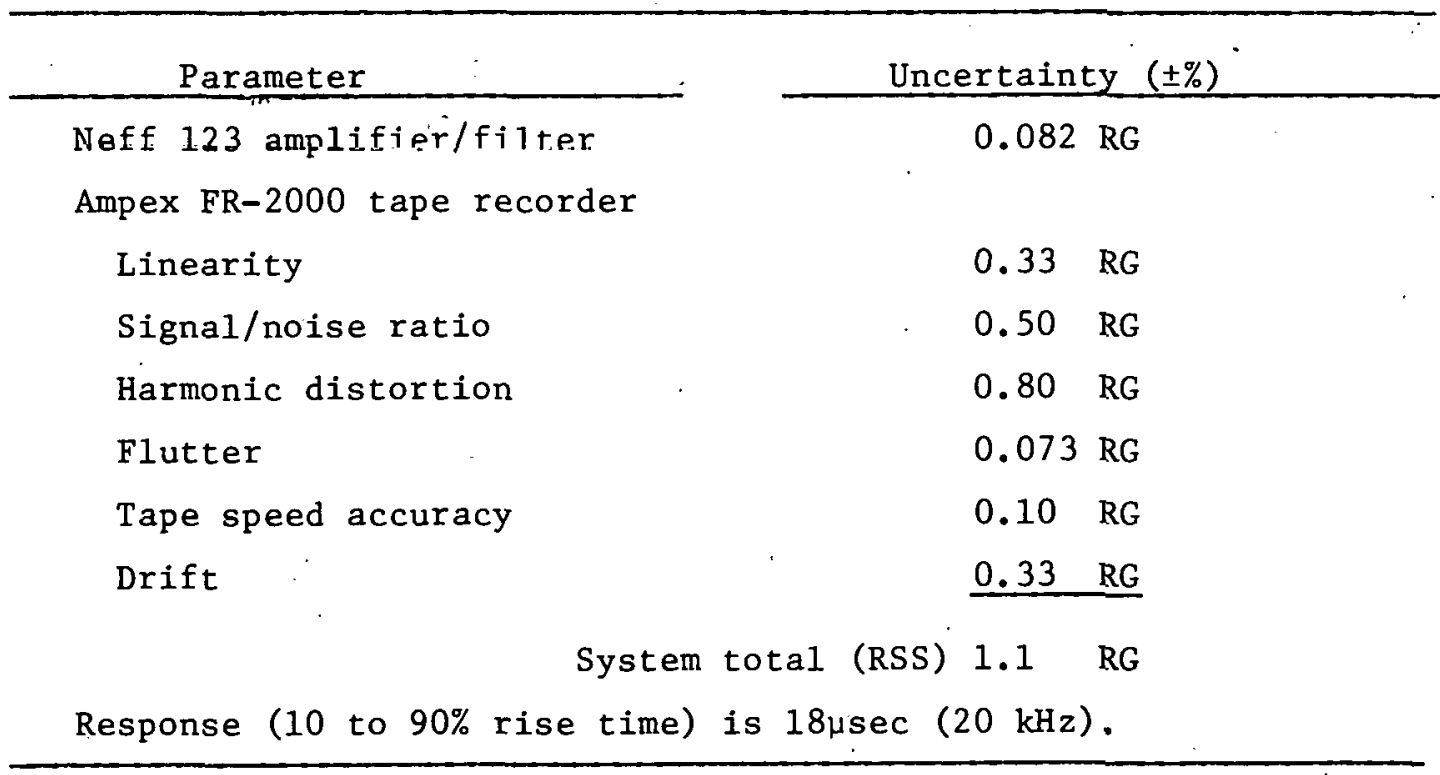


SUMMARY OF MFM UNCERTAINTY ANALYSIS

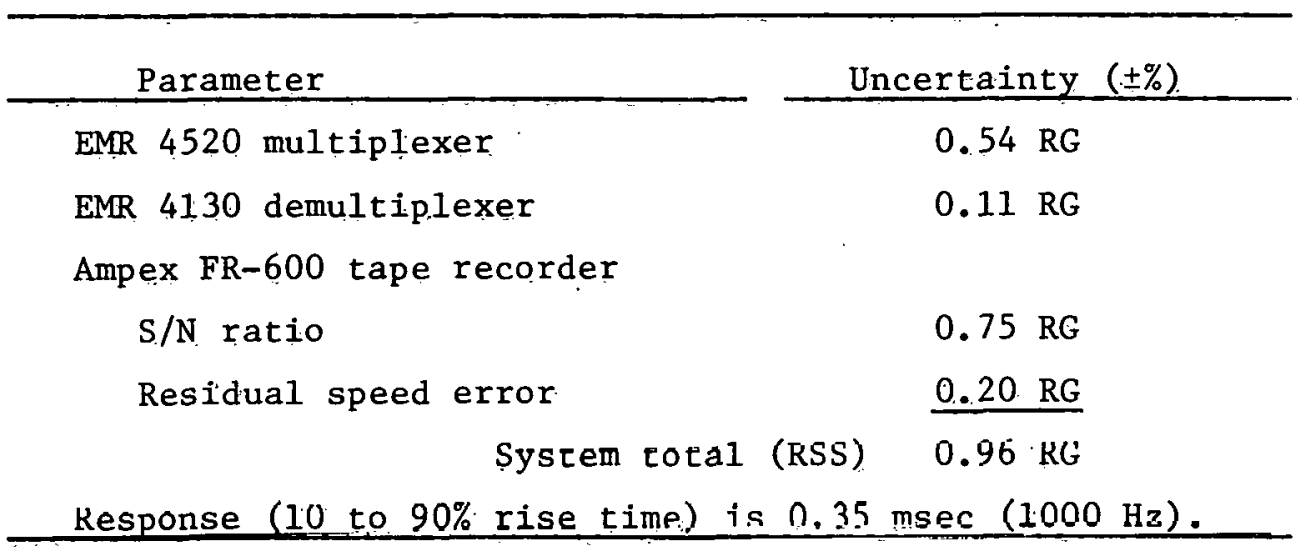

TADLE II-III

* SUMmary of DDaPS UNCERTAINTY ANALYSis

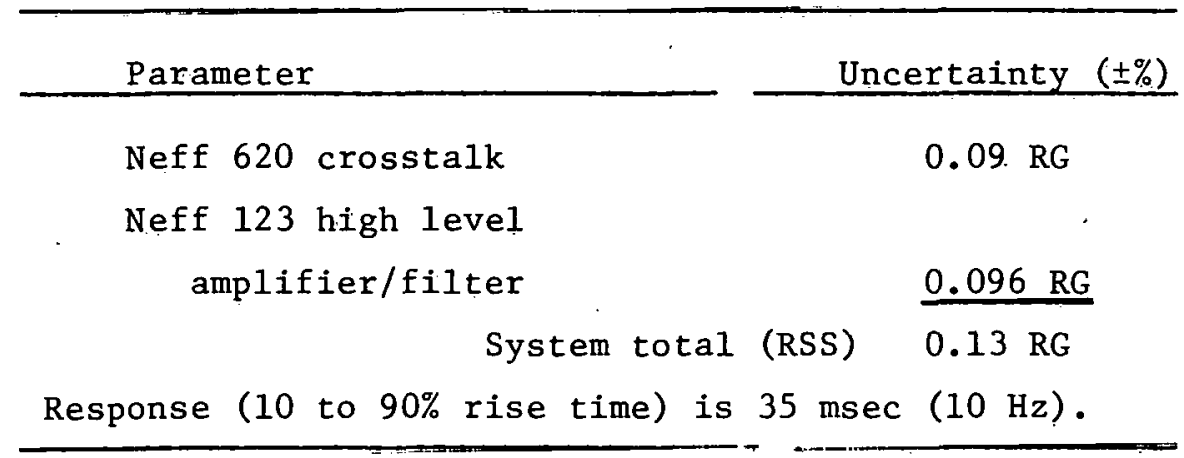

\subsubsection{Voltage Insertion and Shunt Calibration System.}

(1) Voltage Insertion Calibration System. The measurement uncertainties of the voltage insertion calibration system are primarily a function of the various measurement tolerances and noise sources of the electronic equipment used. The voltage insertion signal is supplied by the digital voltage source (DVS) and is fed through the appropriate electronics for recording as a reference. The DVS șupplies signals to all three resnrding sılugystems: the wide band, the narrow band, and the medium band. Since the resnrded voltage calibration values will be used as the reference, the calibration uncertainty must also include all the uncertainty values of the amplifiers, voltage controlled oscillator, and recording devices used. Each of the three recording subsystems are covered individually, since the largest portion of the calibration uncertainty comes from the recording device, not the DVS. The uncertainty values have been calculated based on manufacturers' specifications. Through computer software processing, it may be possible to reduce these uncertainty values. 
(a) Wide-Band Subsystem (WBFM). This subsystem consists of three basic parts, the digital voltage source (DVS) with its attenuator, the Neff 123 amplifier, and the FR-2000 wide-band tape recorder. Using the component specifications, the uncertainty values listed in Table II-IV were derived.

\section{TABLE II-IV}

SUMMARY OF WBFM VOLTAGE INSERTION CALIBRATION

UNCERTAINTY ANALYSIS

\begin{tabular}{lcc}
\hline Parameters & Uncertainty $( \pm \%)$ \\
\cline { 1 - 1 } DVS (HP6130B) & $0.02 \mathrm{RG}$ \\
DVS attenuator & $0.11 \mathrm{RG}$ \\
Neff 123 amplifier & $0.017 \mathrm{RG}$ \\
FR-2000 tape recorder & $\frac{1.1 \mathrm{RG}}{}$ \\
& System total (RSS) & $1.1 \mathrm{RG}$ \\
\hline
\end{tabular}

(b) Multiplexed FM (MFM). This system consists of the DVS with attenuator, a voltage controlled oscillator (VCO) with attenuator, and the tape recorder. Two different VCOs with attenuator are used in the medium-band subsystem, so two different sets of uncertainty values are calculated. In these subsystems, the DVS will be driving four VCOs at one time. The loading effects of the VCO inputs on the VCO attenuators and the loading effects of the DVS attenuator on the four VCO attenuator inputs are considered. The tabulated uncertainties are as listed in Table II-V.

$\underline{\text { TABLE II-V }}$

SUMMARY OF MFM VOLTAGE INSERTION CALIBRATION

SYSTEM UNCERTAINTY ANALYSIS

\begin{tabular}{|c|c|c|c|c|}
\hline Parameter (EMR VCO) & $\begin{array}{c}\text { Uncertainty } \\
( \pm \%) \\
\end{array}$ & $\begin{array}{l}\text { Parameter } \\
\text { (DCS VCO) }\end{array}$ & $\begin{array}{c}\text { Uncertain } \\
\qquad( \pm \%) \\
\end{array}$ & \\
\hline DVS (HP6130B) & $0.02 \mathrm{RG}$ & DVS (HP6130B) & 0.02 & RG \\
\hline DVS attenuator accuracy & $0.11 \mathrm{RG}$ & DVS attenuator accuracy & 0.11 & RG \\
\hline VCO attenuator loading & $0.32 \mathrm{RG}$ & vco attenuator loading & 0.032 & RG \\
\hline vco attenuator accuracy (est.) & ) $0.05 \mathrm{RG}$ & vCO attenuator accuracy & 0.05 & RG \\
\hline VCO loading & $0.20 \mathrm{RG}$ & VCO loading & 0.04 & RG \\
\hline vCO accuracy & $0.54 \mathrm{RG}$ & vCO accuracy & 0.075 & RG \\
\hline vCO demodulator accuracy & $0.11 \mathrm{RG}$ & vCO demodulator accuracy & 0.28 & RG \\
\hline FR-600 tape recorder & $0.78 \mathrm{RG}$ & FR-600 tape recorder & $\underline{0.78}$ & $\underline{R G}$ \\
\hline System total (RSS) & 1.0 RG & System total (RSS) & 0.84 & RG \\
\hline
\end{tabular}


(c) DDAP. With the DVS connected to the subsystem, the overall uncertainty is low. The DDAPS consists of two basic parts: (a) the Neff 620 analog processor and (b) the MODCOMP II/45 computer system. Table II-VI is a summary of the uncertainty.

$\underline{\text { TABLE . II-VI }}$

SUMMARY OF DDAPS VOLTAGE INSERTTON SYSTEM

UNCERTAINTY ANALYSIS

\begin{tabular}{lc}
\hline DDAPS Parameters & Uncertainty $( \pm \%)$ \\
\hline DVS (IIP6130B) & $0.02 \mathrm{KG}$ \\
DVS attenuator & $0.11 \mathrm{RG}$ \\
Nett 620 amplifier & $0.11 \mathrm{RG}$ \\
A/D converter & $\underline{0.003 \mathrm{RG}}$ \\
System total (RSS) & $0.16 \mathrm{RG}$ \\
\hline
\end{tabular}

(2) Shunt Calibration System. A shunt calibration technique is used in LOFT for most of the bridge-type measurement systems (absolute and differential pressure and strain gage). When shunt calibration is done in conjunction with precise knowledge of a series of plant operating pressures (strains are assumed to be at zero reference strain as mounted), a complete channel calibration is possible (gain and offset of transducer and electronics). This technique provides confirmation of transducer, bridge, and electronics operability even when plant conditions are not known.

There are four shunt calibration configuratinns thạt are described:

(1) 3-mV/V full-bridge pressure transducers

(2) $2-\mathrm{mV} / \mathrm{V}$ full-bridge pressure transducers

(3) SG 325 and SG 428 half-bridge strain gages

(4) SG 125 quarter-bridge strain gages.

While there is no specification with which to compare the valucs of uncertainties that are tabulated in this section, the shunt calibration uncertainty $(0.1 \%)$ should not compromise any of the experimental measurements.

(a) Full-Bridge Pressure Transducers. One method of measuring absolute preșsure during LOFT blowdown tests will utilize Taber pressure transducers and associated SRC/Moxon Model 3579A signal conditioners. The Taber bridges are voltage driven and are operated in a four-active arm configuration. These units are calibrated via .a single shunt which is switched across one of two adjacent bridge legs

- to provide a calibration output with reversible polarity. 
The uncertainty in the calibration states was analyzed with a linear error propragation technique and was found to be $0.10 \mathrm{RD}$ for all calibration states. Table II-VII lists the resistor shunts along with the calibration uncertainty.

TABLE II-VII

SUMMARY OF $3-\mathrm{mV} / \mathrm{V}$ FULL-BRIDGE SHUNT

CALIBRATION UNCERTAINTY ANALYSIS

\begin{tabular}{lcc}
\hline $\begin{array}{c}\text { Resistor } \\
\text { Shunt } \\
\text { (ohms) }\end{array}$ & $\begin{array}{c}\text { Standard } \\
\text { Deviation } \\
\text { (ohms) }\end{array}$ & $\begin{array}{c}\text { Uncertainty } \\
( \pm \%)\end{array}$ \\
38,080 & 5.8 & $0.1 \mathrm{RD}$ \\
58,250 & 7.7 & $0.1 \mathrm{RD}$ \\
116,580 & 11.7 & $0.1 \mathrm{RD}$ \\
\hline
\end{tabular}

(b) 2-mV/V Full-Bridge Pressure Transducers. Some of the BaldwinLima-Hamilton (BLH) differential pressure transducers have a sensitivity of $2 \mathrm{mV} / \mathrm{V}$ iristead of $3 \mathrm{mV} / \mathrm{V}$. The analysis of their calibration is identical to the $3-\mathrm{mV} / \mathrm{V}$ full-bridge transducers described in the preceding section with the exception that the shunt calibration resistors have different values. Table II-VIII lists the resistor shunts along with the calibration uncertainty.

TABLE II-VIII

SUMMARY OF 2-mV/V FULL-BRIDGE SHUNT

CALIBRATION UNCERTAINTY ANALYSIS

\begin{tabular}{ccc}
\hline $\begin{array}{c}\text { Resistor } \\
\text { Shunt }\end{array}$ & $\begin{array}{c}\text { Standard } \\
\text { Deviation } \\
\text { (ohms) }\end{array}$ & $\begin{array}{c}\text { Uncertainty } \\
( \pm \%)\end{array}$ \\
\hline 43,555 & 8.7 & $0.1 \mathrm{RD}$ \\
58,250 & 11.7 & $0.1 \mathrm{RD}$ \\
87,325 & 17.5 & $0.1 \mathrm{RD}$ \\
174,825 & 35.0 & $0.1 \mathrm{RD}$ \\
\hline
\end{tabular}


(c) SG 325 and SG 428 Half-Bridge Strain Gages. Three-wire, half-bridge strain gage transducers are utilized in LOFT in conjunction with B \& F Instruments Model DC2473 signal conditioners. The bridge is voltage fed and is shunt calibrated. Table II-IX lists the resistor shunts along with the calibration uncertainty.

TABLE II-IX

SUMMARY OF HALF-BRIDGE SHUNT

CALIBRATION UNCERTAINTY ANALYSIS

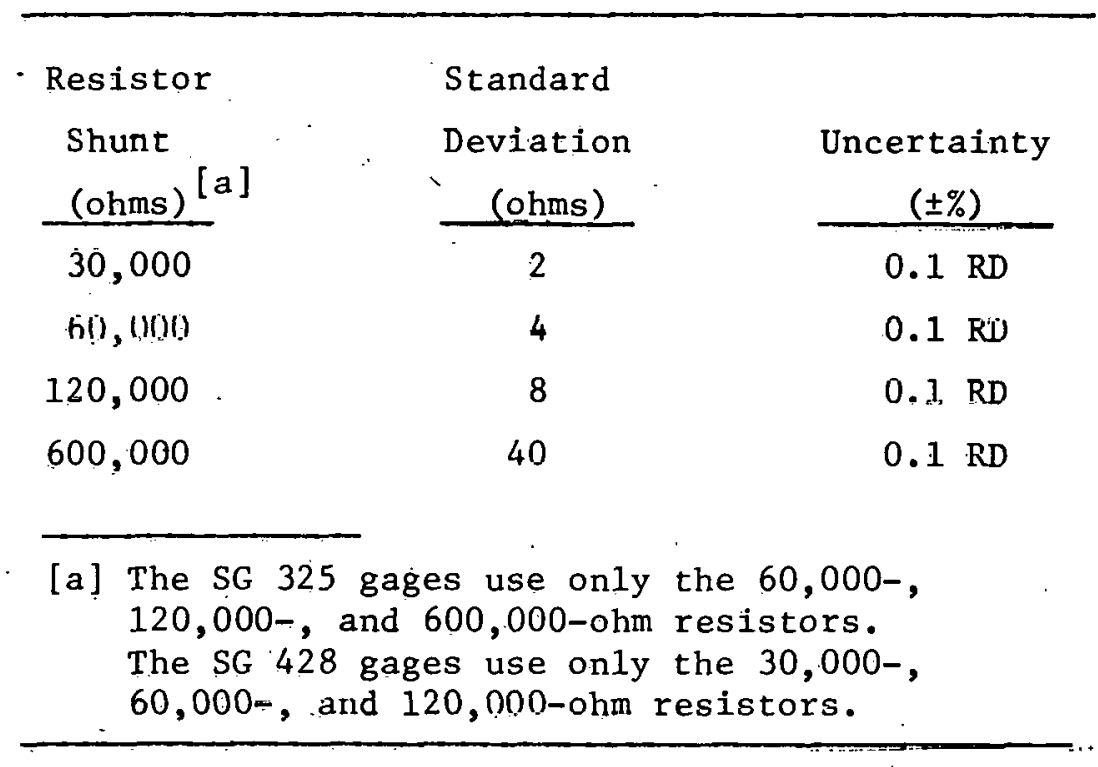

(d) SG 125 Quarter-Bridge Strain Gages. A number of three-wire, quarter-bridgc strain gages are used in LOFT along with their associated B \& F Instruments PC 2471 strain gage signal conditioners. Table II-X lists the resistor shunts along with the calibration uncertainty.

TABLE II-X

SUMMARY OF QৃUARTER-BRIDGE EIIINT

CALIBRATION UNCERTAINTY ANALYSIS

\begin{tabular}{ccc}
\hline $\begin{array}{c}\text { Resistor } \\
\text { Shunt } \\
\text { (ohms) }\end{array}$ & $\begin{array}{c}\text { Standard } \\
\text { Devialiun } \\
\text { (ohms) }\end{array}$ & $\begin{array}{c}\text { Uncertainty } \\
( \pm \%)\end{array}$ \\
60,000 & 4 & $0.1 \mathrm{RD}$ \\
120,000 & 8 & $0.1 \mathrm{RD}$ \\
600,000 & 40 & $0.1 \mathrm{RD}$ \\
\hline
\end{tabular}


1.2.3 Liquid Level Transducer (LLT). Liquid level measurements are made in the core using transducers consisting of spaced arrays of conductivity-sensitive elements. These measurements are used to establish liquid level during reflood and indicate the liquid level variation as a function of time.

The LLT measuring system consists of a conductivity-sensitive transducer array, special electronics package, and DAVDS medium-band system. The presence or absence of liquid is determined by the voltage drop across the media between the electrode and ground plane. A discrimination voltage is chosen which establishes the absence or presence of liquid.

Because of this discrete nature of information to be processed from the LLT output, the common mode interference $( \pm 1 \mathrm{~V}$ peak) and the DAVDS MFM uncertainty does not contribute significantly to the noise margin for the binary "covered - not covered" decision. Therefore, the uncertainty of this measurement consists primarily of the electrode spacing. (See Table II-XI.)

TABLE II-XI

SUMMARY OF LLT UNCERTAINTY ANALYSIS

\begin{tabular}{|c|c|c|}
\hline & Leve1 & \\
\hline & Uncertainty & 10 to $90 \%$ \\
\hline LLT Type & $( \pm \%)$ & Rise Time \\
\hline Core & $2.9 \mathrm{RG}$ & $340 \mathrm{msec}$ \\
\hline Downcomer & $4.5 . R G$ & $340 \mathrm{msec}$ \\
\hline Lower plenum & $7.1 \mathrm{RG}$ & $340 \mathrm{msec}$ \\
\hline
\end{tabular}

The liquid level percent range accuracy requirements are as follows: (a) $3.0 \mathrm{RG}$ for the core, (b) 4.6 RG for the downcomer, and (c) 6.7 RG for the lower plenum. Therefore, the actual liquid level accuracies are within or very close to those required. The response of thc LLT (340 meec) is within the 500-mser requirement.

1.2.4 External Accelerometers. The evaluation of analysis techniques for predicting the response of reactor structures is essential for design and analysis of reactors and reactor safety systems. Accelerometers are one type of transducer used to obtain data for this evaluation.

Accelerometers used in LOFT are the piezoelectric type manufactured by Gulton/ Servonic. The output of the accelerometer goes to a charge amplifier whose output is recorded on the DAVDS (WBF) channels.

The measurement uncertainty analysis showed that the major sources of lerror in the accelerometers are the transducer's transverse sensitivity and calibration. Base strain uncertainty decreases the accuracy of two measurements, while the balance of the accelerometers is isolated from change in base strain. A summary of accelerometer uncertainties is given in Table II-XII. 
TABLE II-XII

SUMMARY OF EXTERNAL ACCELEROMETER UNCERTAINTY ANALYSIS

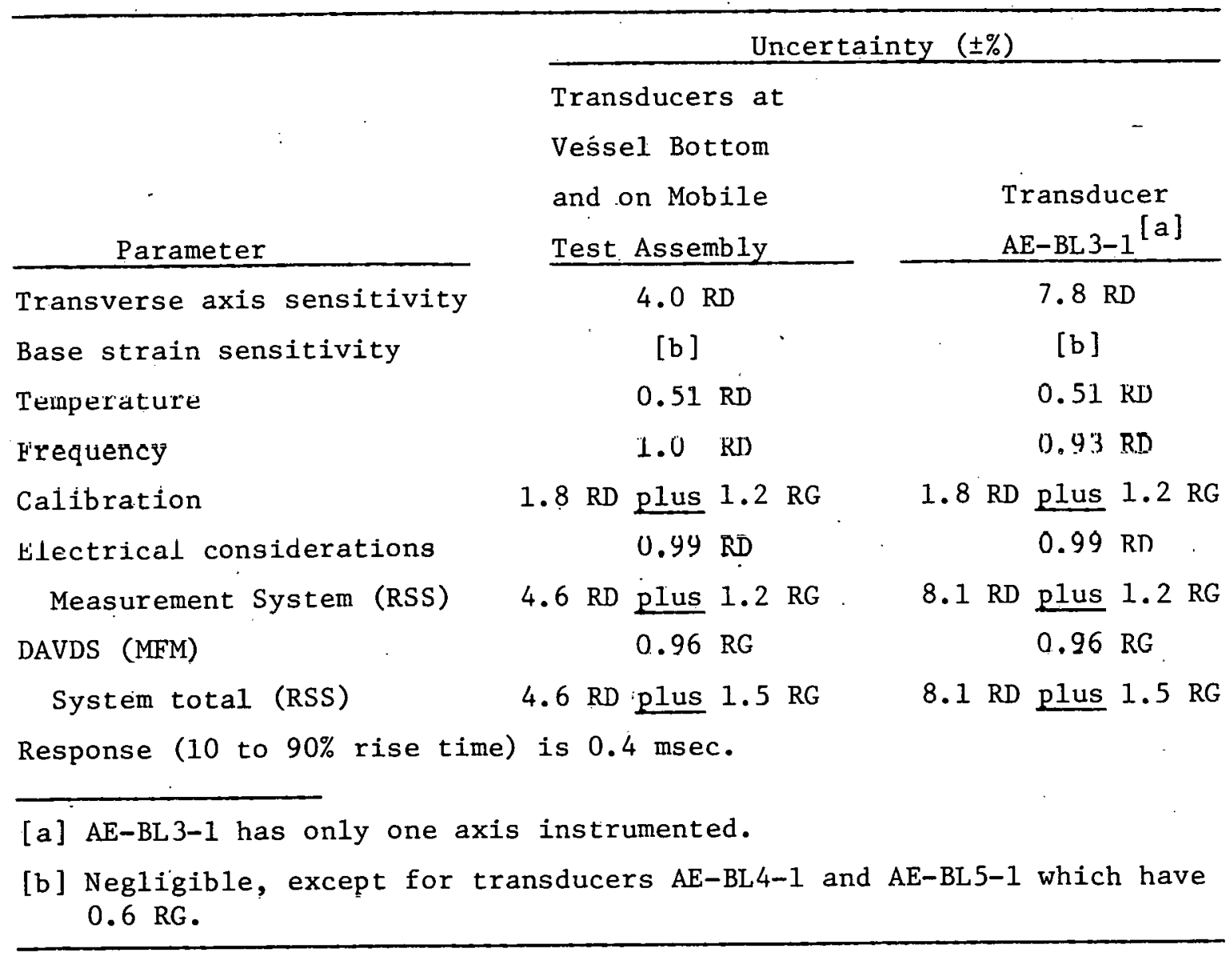

The accuracy requirement for accelerometers is 10 RD. Table II-XII shows that LOFT experimental accelerometer accuracies are well within this requirement.

1.2.5 Linear Variable Differential Transformer (LVDT). Two LVDT displacement transducers are used to measure the thermal expansion and vertical displacement of the. central fuel assembly during a loss-of-coolant experiment (LOCE). The maximum vertical excursion that can occur is slightly. less than 1 inch. The response time estimate for the LVDTs has been determined by analysis to be $6.4 \mathrm{msec}$. The total uncertainty of the LVDT displacement measurements is primarily a function of the mounting alignment and the linearity of the LVDT. The uncertainty in the mounting alignment will not exceed $2.2 \mathrm{RG}$. The linearity uncertainty of the LVDTs has been tested. Using a straight line best fit on the test data shows a worst case linearity fit of $3.5 \mathrm{RD}$. A summary of uncertainties is shown in Table II-XIII. The range of the LVDT is \pm 0.5 inch. 
TABLE II-XIII

SUMMARY OF LVDT UNCERTAINTY ANALYSIS

\begin{tabular}{|c|c|}
\hline Parameter & Uncertainty $( \pm \%)$ \\
\hline \multicolumn{2}{|l|}{ Calibration } \\
\hline Micrometer & $1.0 \mathrm{RG}$ \\
\hline Carrier amplifier & $2.0 \mathrm{RG}$ \\
\hline Linearity & $3.5 \mathrm{RD}$ \\
\hline Measurement channel (RSS) & 2.2 RG plus $3.5 \mathrm{RD}$ \\
\hline DAVDS (MFM) & $0.96 \mathrm{RG}$ \\
\hline System total (RSS) & $2.4 \mathrm{RG}$ plus $3.5 \mathrm{RD}$ \\
\hline Response ( 10 to $90 \%$ rise tim & $6.4 \mathrm{msec}$. \\
\hline
\end{tabular}

No performance requirements are established for the LVDTs since these measurements are primarily requalification measurements. The uncertainties on the LVDT displacement transducers are low enough that usable data will be obtained from the measurements.

1.2.6 Self-Powered Neutron Detectors (SPND). The core thermal neutron power history will be obtained during 'LOCEs using fixed position, self-powered nuclear detectors.

This measurement system consists of:

(1) A.neutron sensitive cobalt detector

(2) An autoranging picoammeter

(3) DAVDS multiplexed FM recording channel.

The requirement established by the system design description (SDD) for nuclear detector measurement is $5 \mathrm{RD}$. Therefore, the actual accuracy of $4.5 \mathrm{RD}$ is within the requirement, but the $3.9 \mathrm{RG}$ causes the total uncertainty to exceed $5 \mathrm{RD}$ at all power levels. If the decay heat experiment is performed, the total uncertainty is $0.27 \mathrm{RD}$ plus $0.20 \mathrm{RG}$. Under this condition, the total uncertainty would not exceed $5 \mathrm{RD}$ above $4 \%$ of full power. A summary of SPND uncertainties is given in Table II-XIV.

1.2.7 Traversing In-Core Probe (TIP). Axial thermal neutron flux profiles are measured in LOFT using the TIP system. Flux profiles are recorded under steady state conditions prior to a LOCE.

The TIP system is a complete system that was purchased from the General Electric Company. The system is comprised of two separate subsystems: (a) a cable device and storage system and (b) a system contrul and data processing systcm. 
TABLE II-XIV

SUMMARY OF SPND UNCERTAINTY ANALYSIS

\begin{tabular}{|c|c|c|}
\hline Parameter & $\begin{array}{l}\text { Uncertainty }( \pm \%) \\
\left(10^{-9} \text { A Range) }\right. \\
\end{array}$ & $\begin{array}{l}\text { Uncertainty }( \pm \%) \\
\text { (Other Range) }\end{array}$ \\
\hline Gamma sensitivity & 4.5 RD plus $3.3 \mathrm{RG}$ & $4.5 \mathrm{RD}$ plus $3.3 \mathrm{RG}$ \\
\hline Calibration repeatability & $0.4 \mathrm{RD}$ & $0.4 R D$ \\
\hline Electronics error (est) & $2.1 \quad \mathrm{RG}$ & $1.1 \mathrm{RG}$ \\
\hline Measurement Channel (RSS) & 4.5 $\mathrm{RD}$ plus $3.3 \mathrm{RG}$ & $4.5 \mathrm{RD}$ plus $3.5 \mathrm{RG}$ \\
\hline DAVDS (MFM) & $0.13 \mathrm{RG}$ & $0.13 \mathrm{RG}$ \\
\hline System total (RSS) & $4.5 \mathrm{RD}$ plus $3.9 \mathrm{RC}$ & $4.5 \mathrm{RD} \Gamma 111 \mathrm{~S} 3.5 \mathrm{DG}$ \\
\hline \multicolumn{3}{|c|}{$\begin{array}{l}\text { Response ( } 10 \text { to } 90 \% \text { rise time) over the range } 10^{-5} \text { to } 10^{-8} \text { is } 35 \mathrm{msec} \\
\text { and for maximum range } 10^{-9} \text { is } 38 \mathrm{msec} \text {. } \\
\text { Position uncertainty is } \pm 0.051 \text { inch. }\end{array}$} \\
\hline
\end{tabular}

The major source of uncertainty in the TIP system is the detector gamma sensitivity and detector/electronics linearity, hysteresis, and repeatability. Data are recorded on an X-Y plotter provided as part of the system and on the DAVDS. A summary of the TIP system is given in Table.II-XV.

TABLE II-XV

SUMMARY OF TIP UNCERTAINTY ANALYSIS

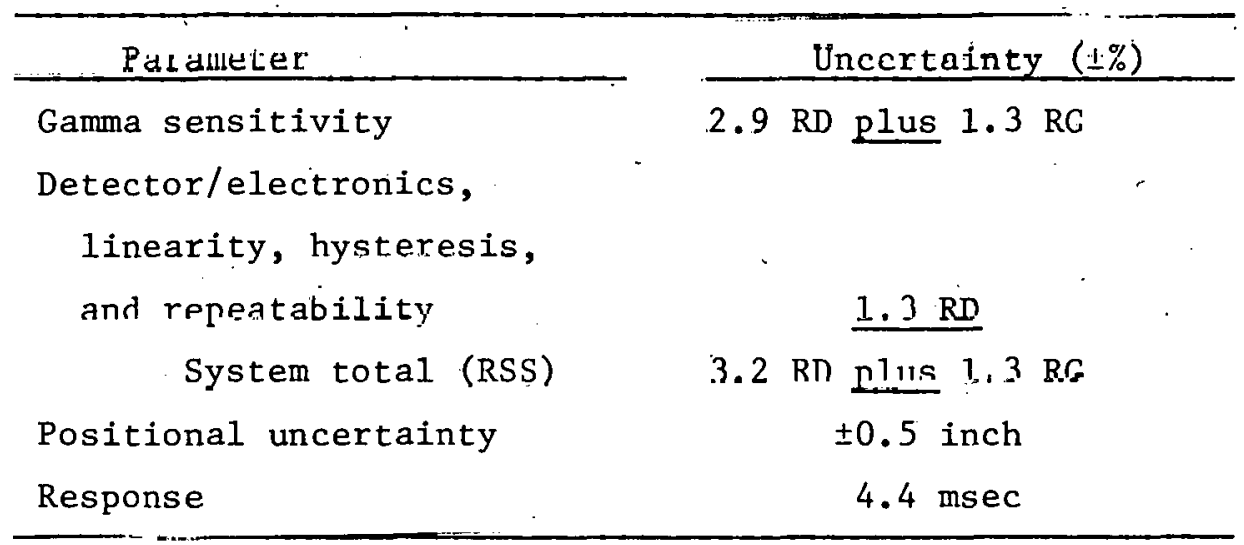

An accuracy of $2.0 \mathrm{RD}$ of reading was established for the TIP system as an objective. The actual uncertainty of $3.2 \mathrm{RD}$ plus 1.3 RG does not meet this specification. With performance of a decay power calibration experiment on LOFT, the inaccuracy resulting from gamma sensitivity of the fission chamber can be minimized; and the measurement uncertainty would then reduce to $1.3 \mathrm{RD}$ plus $0.07 \mathrm{RG}$, which meets the system objective. 
1.2.8 Strain Gage. The strain gage measurements use 170 transducers for obtaining dynamic strain measurements on the reactor piping and mechanical structure during a LOCE. These strain measurements are to be used to determine dynamic loading conditions on the LOFT Integral Test System.

The largest single source of uncertainty, outside of the manufacturer's specifications, is caused by the mechanical alignment of the strain gages. The next largest source of uncertainty is produced in the gage factor by the temperature change from room temperature to the steady state operating temperature. If the operating temperature is known to within $\pm 100^{\circ} \mathrm{F}$, the uncertainty value is within requirements.

The strain gages are electrically connected to Wheatstone bridge networks through long cable lengths. The resistance of the cable will produce an uncertainty in the gage factor and bridge balance. This is potentially a source of uncertainty but will be carefully reduced by calibration. The calibration procedure requires a recalculation of the gage factor for each strain gage and an estimated temperature operating range for each. With the cable resistance calibration, the uncertainties are $0.5 \mathrm{RD}$. Without the cable resistance calibration, the uncertainties are $10 \mathrm{RD}$. After the calibration, the summary of uncertainties shown in Table II-XVI will be obtained.

TABLE II-XVI

SUMMARY OF STRAIN GAGE UNCERTAINTY ANALYSIS

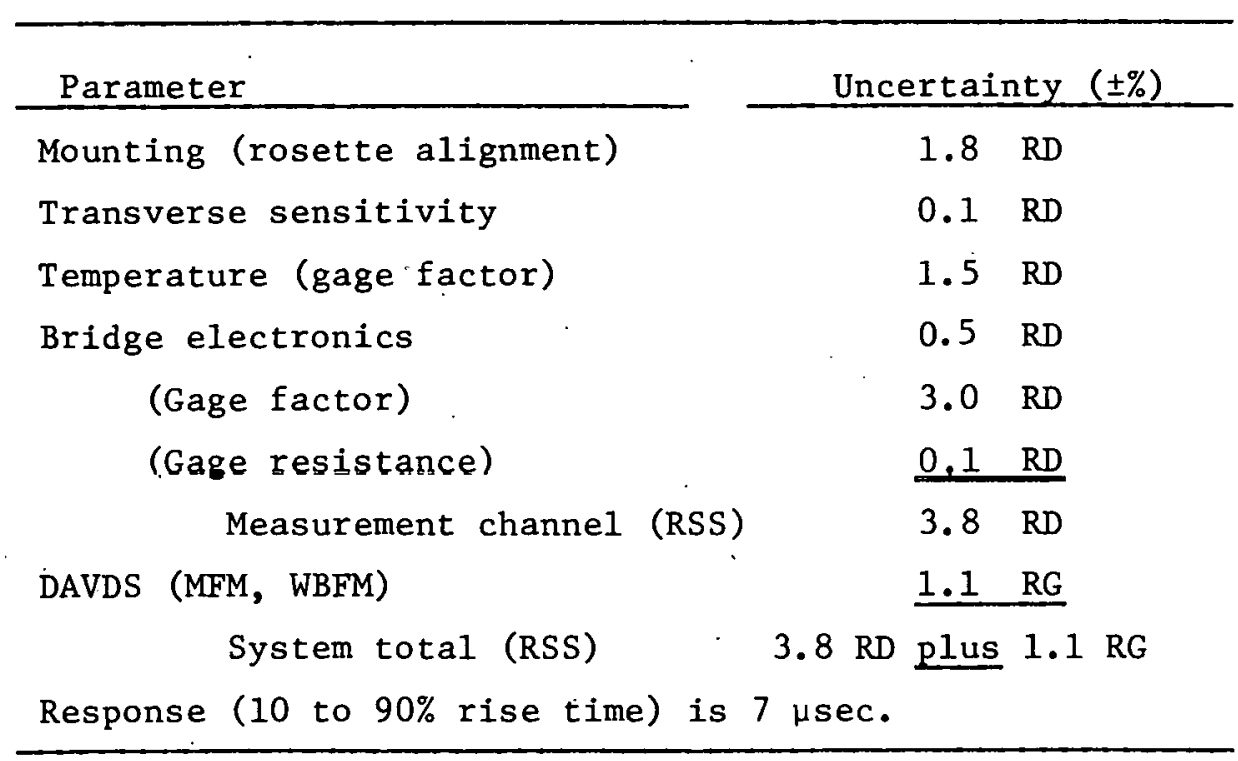

The strain measurements are better than the specified requirements of $10 \mathrm{RD}$ with a response time of $1 \mathrm{msec}$. The uncertainty of $3.8 \mathrm{RD}$ is within the requirements. The recording channel uncertainty of $1.1 \mathrm{RG}$ will become significant at low signal levels. The system response will be controlled by the response time of the DAVDS recording channel used. 
1.2.9 Standoff Absolute Pressure. Absolute pressure measurements fulfill several major functions such as:

(1) Determining steady state pre-LOCE operating conditions

(2) Providing a means of determining pressure loads on the core and core barrel during subcooled decompression

(3) Aiding in the interpretation of flow, differential pressure, and heat transfer measurements obtained during all phases of the LOCE

(4) Aiding in determining the effect of the coolant in the upper plenum on the reflood phenomena in the core.

Absolute pressure measurements in LOFT are accomplished using commercially available pressure transducers and transmission lines or free-field pressure transducers (FFPT) (see 1:2:10 below).

Major sources of uncertainty in pressure measurements are the transmission line effect and the shunt calibration. A summary of the uncertainty in this measurement is given in Table II-XVII.

\section{TABLE II-XVII}

SUMMARY OF STANDOFF ABSOLUTE PRESSURE UNCERTAINTY ANALYSIS

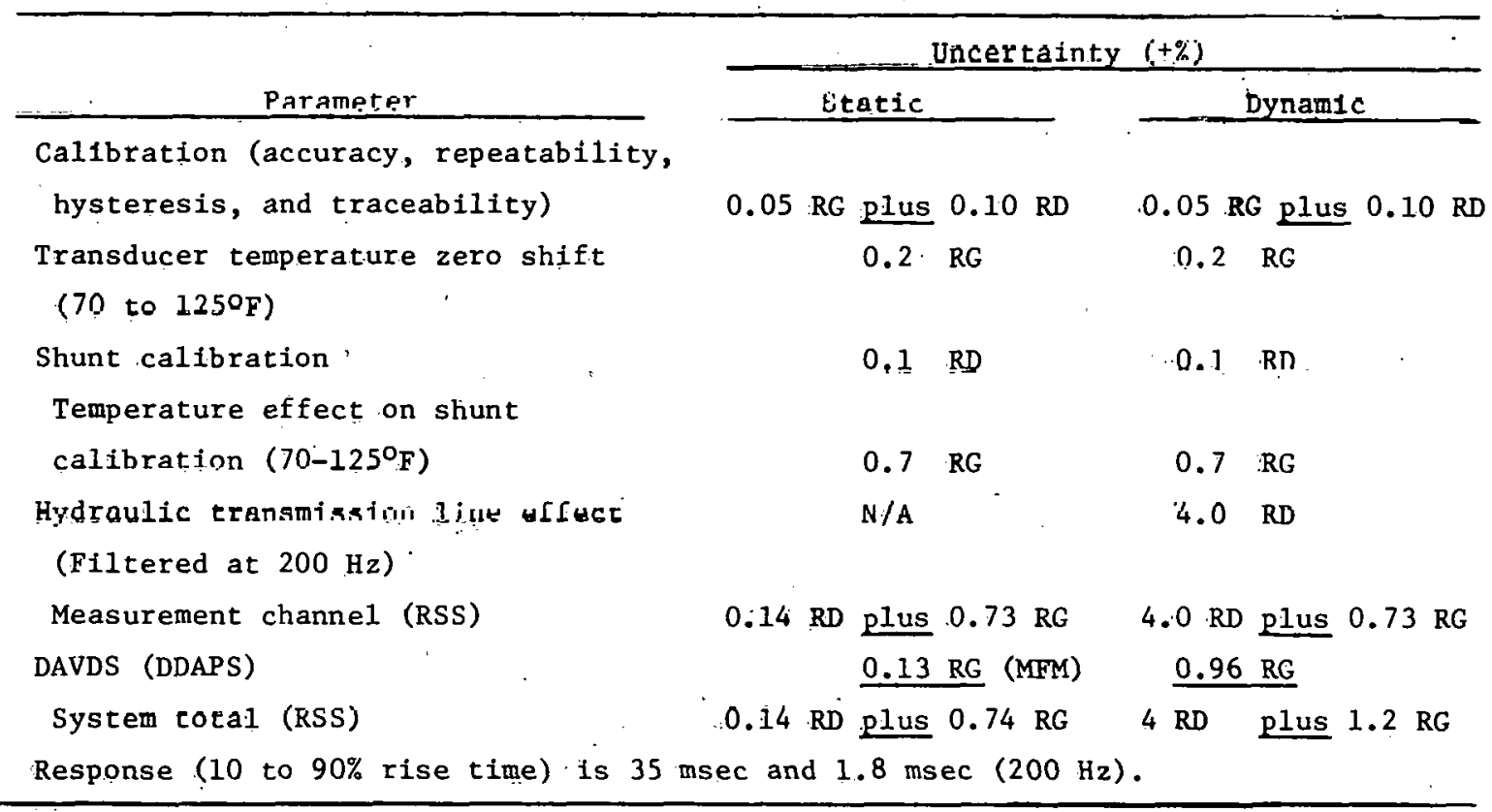


The specified accuracy requirements are $1 \mathrm{RG}$ for subcooled blowdown, $5 \mathrm{RD}$ for saturated blowdown, and \pm 1 psi for reflood. Response requirements are $1 \mathrm{msec}(350 \mathrm{~Hz})$ for subcooled blowdown, $150 \mathrm{msec}(2.3 \mathrm{~Hz})$ for saturated blowdown, and $500 \cdot \mathrm{msec}(0.7 \mathrm{~Hz})$

$\therefore$ for reflood. The actual worst accuracy will be $5.2 \mathrm{RG}$ below $200 \mathrm{~Hz}$. Filtering is required due to the resonant frequency of the standoff which is approximately $220 \mathrm{~Hz}$. This frequency decreases for longer standoffs .(longer than 3 feet). Therefore, the standoff absolute pressure measurements do not meet the response requirement for subcooled blowdown but do meet the requirement for saturated blowdown and reflood.

1.2.10 Free-Field Pressure Transducer. The loads that could accompany decompression must be known and reflected in the reactor design. The pressure loads on the core and core barrel during subcooled decompression in LOFT are measured using FFPTs.

The FFPT is a transducer designed by Aerojet Nuclear Company and consists of a strain post with four active strain gages as the sensing element and which are enclosed by a bellows and two end plates. Signal conditioning is accomplished using commercially available electronics. The FFPT output is recorded on DAVDS WBFM channels.

The major source of uncertainty in the FFPT is the calibration accuracy, which is established by procedural requirements. A summary of the FFPT uncertainties is presented in Table II-XVIII. The range of all FFPTs is 0 to 3,000 psi.

TABLE II-XVIII

SUMMARY OF FFPT UNCERTAINTY ANALYSIS

\begin{tabular}{lc}
\hline Parameter & Uncertainty ( $\pm \%)$ \\
\hline Calibration accuracy & $2.5 \mathrm{RG}$ \\
Hysteresis & $0.75 \mathrm{RG}$ \\
Repeatability & $\frac{4.0 \mathrm{RD}}{\text { Measurement channel (RSS) }} 2.6 \mathrm{RG}$ plus $4.0 \mathrm{RD}$ \\
DAVDS (WBFM) & $\underline{1.1 . \mathrm{RG}}$ \\
System total (RSS) & $2.8 \mathrm{RG}$ plus $4.0 \mathrm{RD}$ \\
Response (10 to 90\% rise time is $85 \mu \mathrm{sec}$. \\
\hline
\end{tabular}

Accuracy requirements of 1 RG were established for subcooled blowdown for the FFPT. Response requirements were specified as $1 \mathrm{msec}$. The observed total uncertainty is 2.8 RG plus 4.0 RD, which does not meet the requirement. The response of the FFPT is well within the $1-\mathrm{msec}$ requirement.

1.2.11 Differential Pressure Transducers. Differential pressure measurements are required in LOFT to provide information on pressure losses and differences. This is 
accomplished using commercially available BLH differential pressure transducers and fluid transmission lines to connect the transducer to the measurement location. The electronics used are commercially available.

The uncertainty in differential pressure measurement is completely dominated by the fluid transmission lines. Table II-XIX is a summary of the differential pressure transducer uncertainty analysis.

TABLE II-XIX

SUTMMARY DIFFERENTIAL PRESSURE TRANSDUCER UNCERTAINTY ANALYSIS

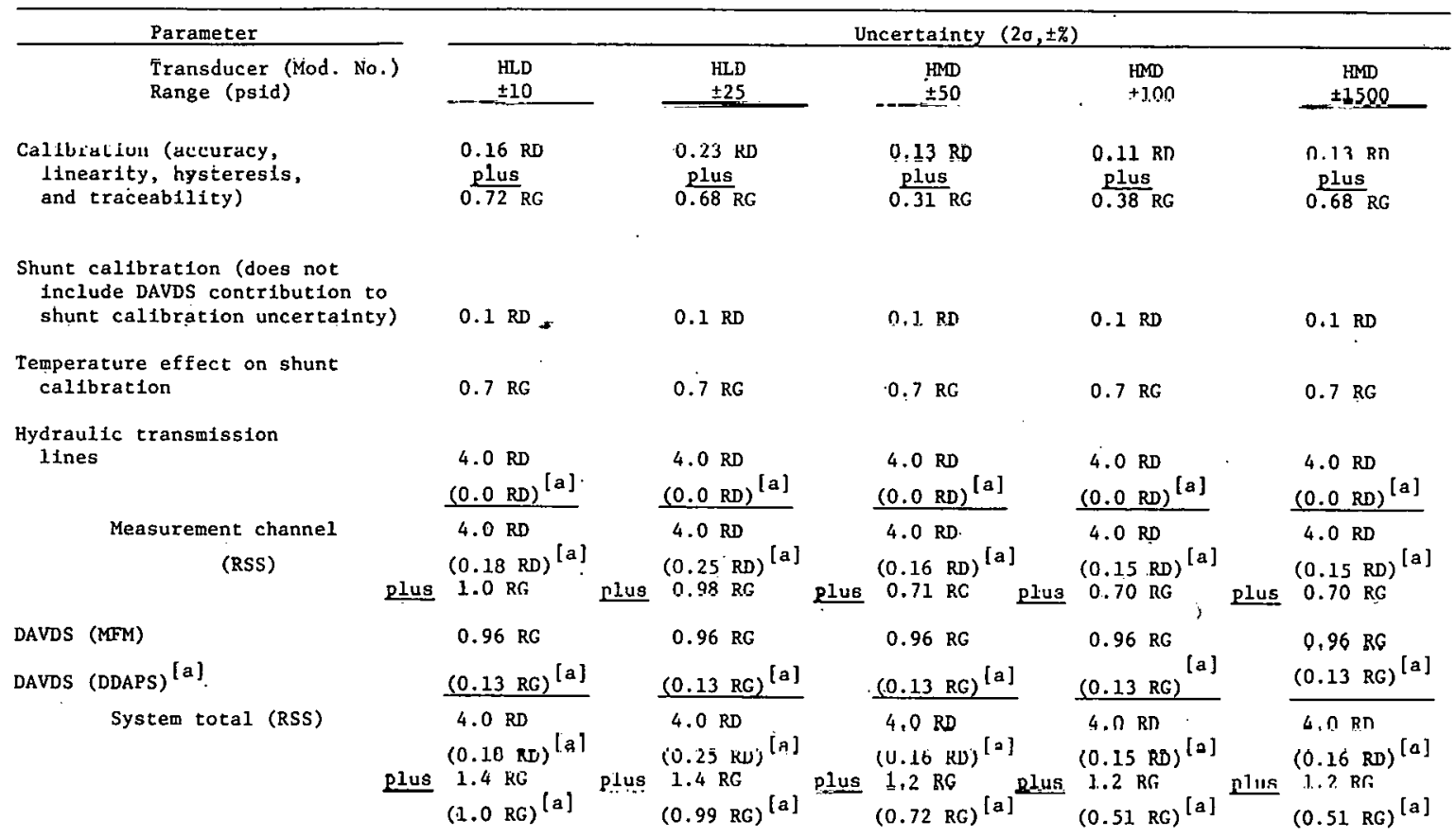

Response ( 10 to $90 \%$ rise time) is established by the extent of filtering required.

[a] Values in parentheses are.for static measurements. Other entries in this table are for dynamic measurements. For static measurements, the uncertainties from hydraulic transmission line effects are eliminated and the nAVhs uncertainties are reduced to $0.13 \mathrm{RG}$, a result of using DDAPS instead of MFM.

The differential pressure transducer itself is $1 \mathrm{RD}$. The major unccrtainty arises from the use of long standoffs to make the pressure measurements. With $200-\mathrm{Hz}$ data filtering assumed, the residual uncertainty in differential pressure measurements outside the reactor vessel should bc about 4 RD and 2 RG. For the seven differential pressure médsurements, all are within or partly within the vessel, and the effect of steam generation in the transmission line causes the uncertainty to rise to about $8 \mathrm{RD}$ and $2 \mathrm{RG}$ for line pressures between 650 and 1,500 psia. Requirements are either $10 \mathrm{RD}$ or $2 \mathrm{RG}$, depending on the specific measurement. Uncertainties are presently undefined for line pressures below 650 psia. Response requirements are $150 \mathrm{msec}, 200 \mathrm{msec}$, and $300 \mathrm{msec}$, again depending on the specific measurement. With correct filtering, the differential pressure transducers will meet the MRD requirements. The effect of long fluid transmission lines or the filtering technique has not been verified. 
1.1.12 Temperature. Experimental temperature measurements are one of the major measurement categories in the LOFT Integral Test System and account for more than 300 discrete measurements. Each of these measurements is made with one resistance thermometer detector (RTD) and one of three different types of thermocouples (TC), viz., Type $\mathrm{K}$, Type $\mathrm{S}$, and Type $\mathrm{T}$. A $150^{\circ} \mathrm{F}$ reference junction is used with all the thermocouples. The RTD and all Type $T$ thermocouples are used for coolant temperature measurements. The Type $S$ thermocouples are used for metal cladding themperatures, and the Type $\mathrm{K}$ thermocouples are used for both coolant and metal cladding temperatures.

The largest uncertainties come from the standard accuracy tolerances of the transducers which result from impurity levels in the thermocouple and connecting lead materials. A calibration equation has been calculated for each of the three types of thermocouples utilizing data from ANSI Standard C96.1. Some additional uncertainty is contributed by the calibration equation, but it is small compared to the standard accuracy uncertainties. Acceptance data were supplied with the thermocouples to be used in LOFT; the data were checked with the standard accuracy tables, and it was confirmed that the thermocouples are within the standard uncertainty bounds.

The uncertainties summarized in Table II-XX pertain primarily to the thermocouples or the RTD and do not include mounting effects.

1.2.13 Drag Disk-Turbine Transducer (DTT). One of the basic quantities of interest during a LOCE is the primary system mass flow rate. The method used in LOFT to obtain mass flow rate is to measure coolant velocity and momentum flux from which mass flow rate can be calculated. This measurement will be accomplished using the DTT, which consists of three transducers mounted in series: a drag disk, a thermocouple, and a turbine, in that order. The drag disk force is translated to an output using a torsion bar and variable reluctance transducer, while the turbine uses an eddy current transducer for a pickup. Special electronics are used with the thermocouple.

A special data reduction technique is used for the DTT turbine in two-phase flow. This technique takes slip and void fraction into account, which allows a more accurate velocity to be calculated.

The major sources of uncertainty in the DTT are the calibration and linearity/ repeatability. A summary of the DTT uncertainty analysis is presented in Tables II-XXI and II-XXII. (Note: The thermocouple uncertainty analysis was included in Section 1.2.12 above.)

The accuracy requirement for the turbine is $5 \mathrm{RD}$, with a response of $100 \mathrm{msec}$. The observed accuracy of $7.9 \mathrm{KG}$ and $220 \mathrm{msec}$ is in excess of this requirement.

No specific requirement for the drag disk accuracy has been established. Density is listed at $5 \mathrm{RD}$. The density may be calculated using the velocity from the turbine and the $\rho v^{2}$ from the drag disk. 


\section{TABLE II-XX}

SUMMAFY OF EXPERIMENTAL TEMPERATURE UNCERTAINTY ANALYSIS

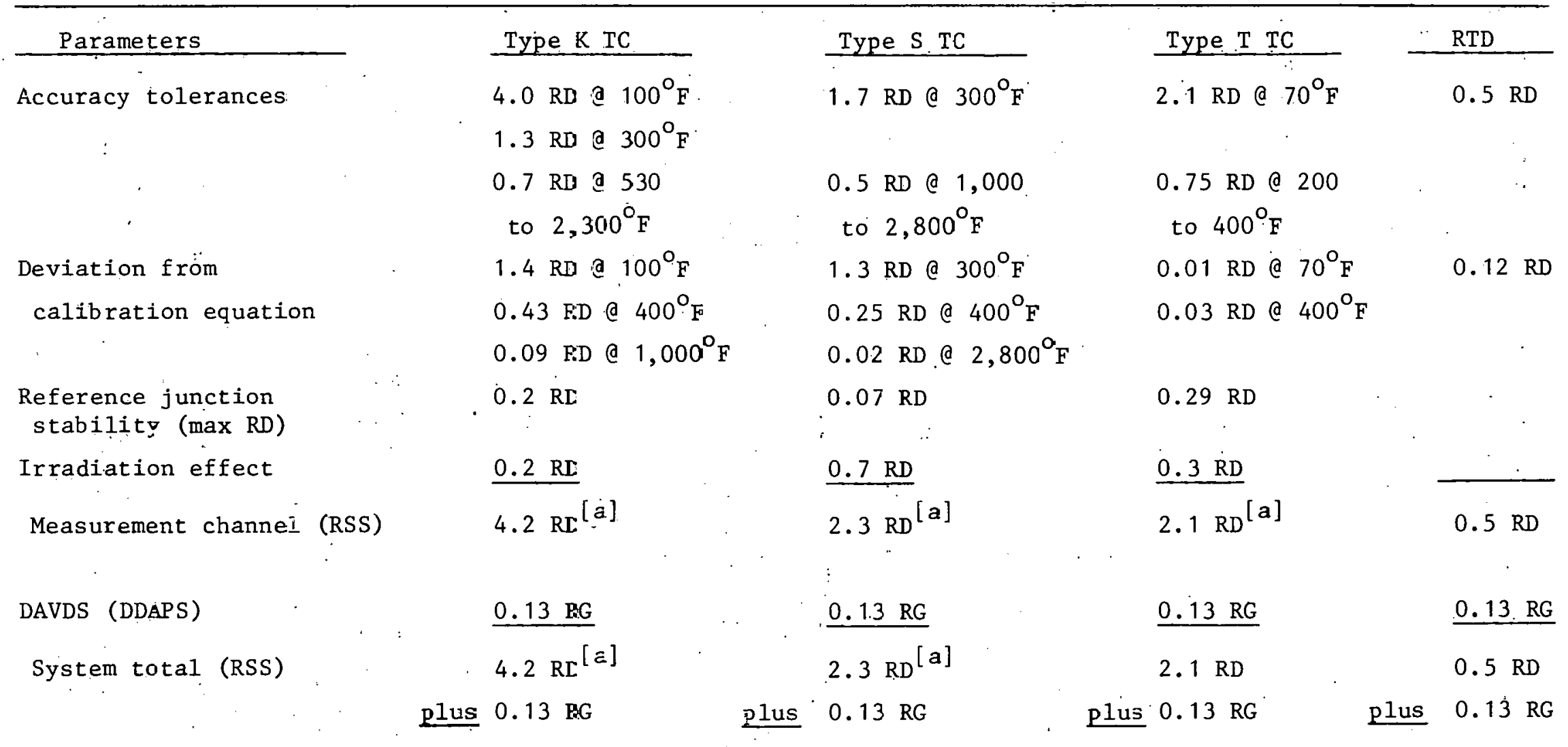

[a] The total uncertainty (RD) is given at wors- case temperatures. Some cf the specific uncertainty values are given at different temperatures in orde= to give additional understanding to the reader. 
TABLE II-XXI

SUMMARY OF DTT TURBINE UNCERTAINTY ANALYSIS

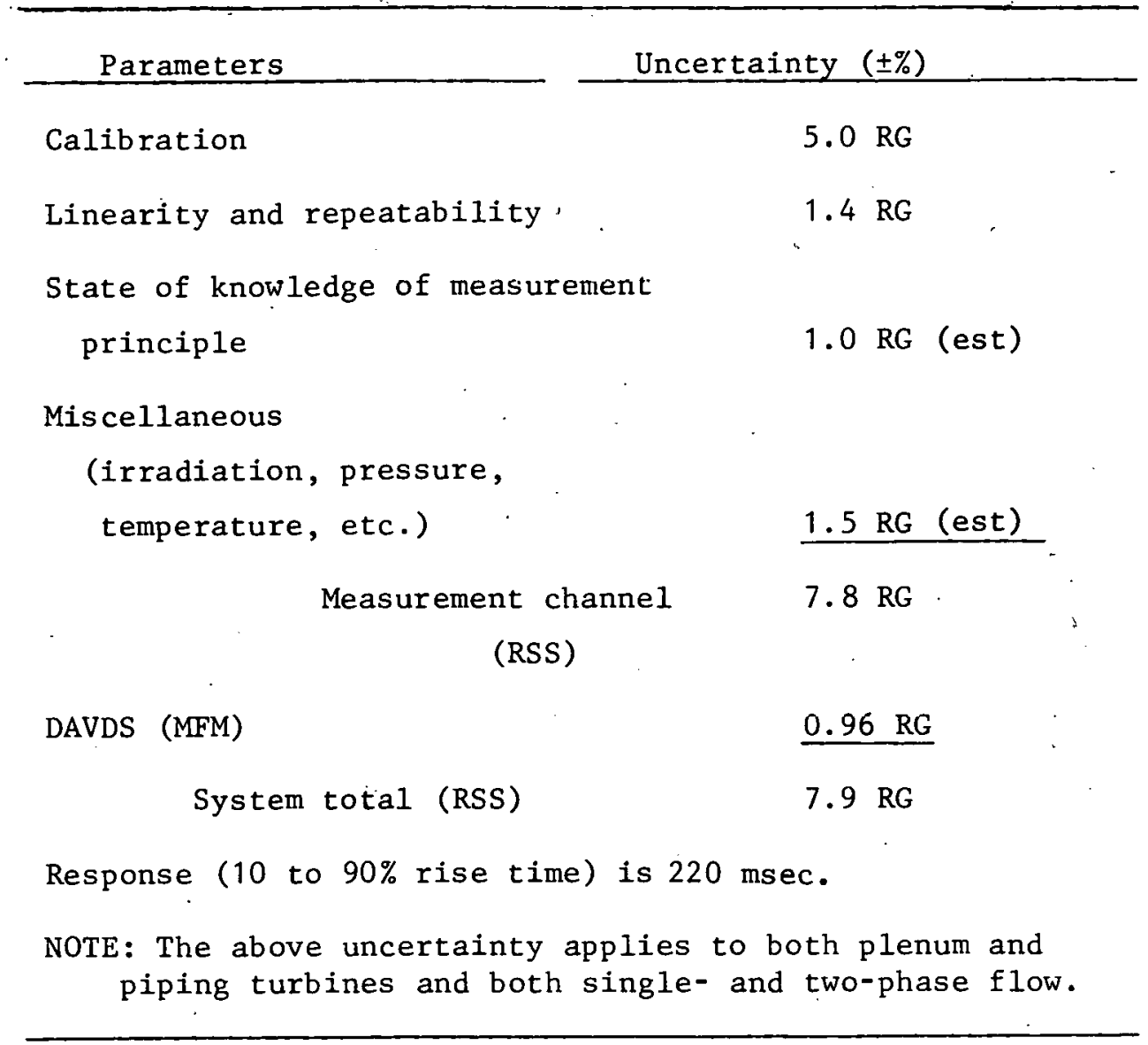

1.2.14 Pump Speed. It is important that the effect of the primary coolant pump on the LOCE transient be obtainable from test data. In order to understand primary coolant pump performance, several variables must be measured. One of these variables is pump speed. In LOFT, this measurement will be made using a Kaman Science Corporation eddy current transducer. The transducer is mounted through the primary coolant pump end cap. A plate with six indentations is placed on the end of the pump shaft. As each indentation passes the eddy current transducer, a variation in the impedance is produced. Kaman electronics are also used for conditioning the signal. This measurement can be recorded as either a pulse train on the MFM system or as a voltage analog of frequency on the DDAPS.

This is a very accurate measurement, and a summary of the uncertainty analysis is presented in Table II-XXIII. All of the entries are from the frequency-to-voltage converter specifications.

If the measurement is recorded as an analog of frequency, the uncertainty will be that of the MFM system (0.96 RG). 
TABLE II-XXII

SUMMARY OF DTT DRAG DISK UNCERTAINTY ANALYSIS

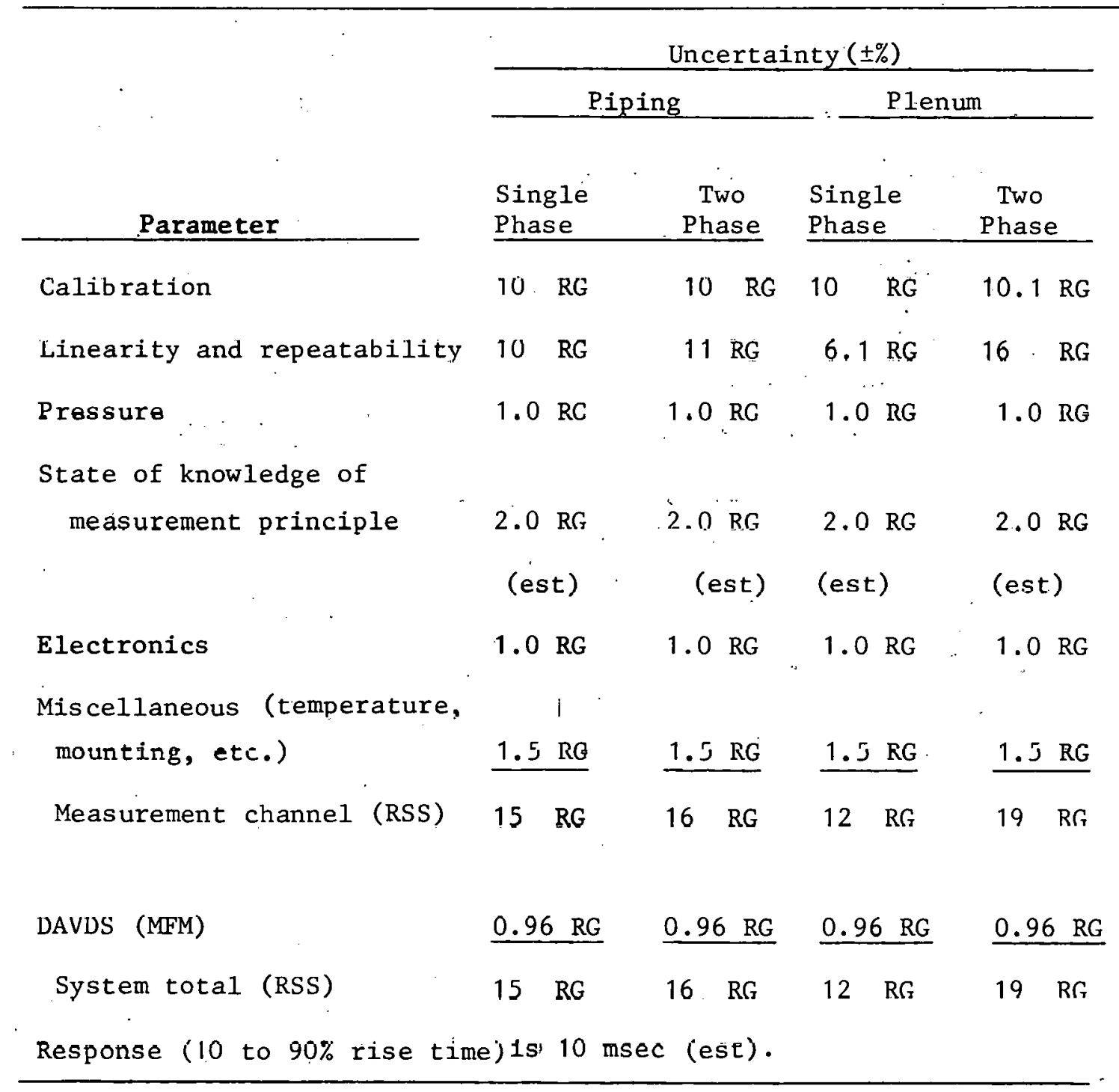

1.2.15 Gamma Densitometer. Knowledge of fluid flow can be obtained from a number of sources. One of the most direct means is by determining the-velncity and density and then calculating the flow. A discussion of this measurement using the DTT is presented in Section 1.2.13. Another method of obtaining density is by using a gamma densitometer. It should be noted that this method is only applicable to nonnuclear testing.

The basic elements of the gamma densitometer system are:

(1) A Cs-137 gamma source in a shielded cask

(2) Three integral scintillation detectors

(3) Three shielded casks for the detectors 
SUMMARY OF PUMP SPEED UNCERTAINTY ANALYSIS

\begin{tabular}{lc}
\hline Parameter & Uncertainty $( \pm \%)$ \\
\hline $\begin{array}{l}\text { Shift in full-scale }\left( \pm 5^{\circ} \mathrm{C}\right) \\
\text { Linearity }\end{array}$ & $0.05 \mathrm{RG}$ \\
Accuracy (gain) & $0.03 \mathrm{RG}$ \\
(offset) & $0.03 \mathrm{RD}$ \\
Ripple & $0.10 \mathrm{RG}$ \\
Measurement channel (RSS) & $\frac{0.22 \mathrm{RD}}{0.22 \mathrm{RD}}$ \\
DAVDS (DDAPS) & $0.12 \mathrm{RG}$ \\
System ,total (RSS) & $0.13 \mathrm{RG}$ \\
Response (10 to 90\% rise time) is $100 \mathrm{msec}$. \\
\hline
\end{tabular}

(4) Three high voltage power supplies

(5) Three preamplifiers

(6) Three amplifier/control units

(7) An actuator control panel.

The summary of the gamma densitometer uncertainty analysis is presented in Table II-XXIV. This analysis is valid for the steady state density of a given beam. The range of the gamma densitometer is 0.5 to $62.4 \mathrm{lb} / \mathrm{ft}^{3}$.

The requirement for average density is $5 \mathrm{RD}$ with a response of $100 \mathrm{msec}$. The single beam results, presented in Table II-XXIV, are only valid for homogeneous flow. An average density data reduction program for yarious other types of flow regimes (slug, annular, etc.) is currently being developed. The actual accuracy, for homogeneous flow, is within the requirements down to $12 \mathrm{lb} / \mathrm{ft}^{3}$. At values lower than $12 \mathrm{lb} / \mathrm{ft}^{3}$, the gamma densitometer homogeneous flow accuracy will not meet the requirements. Response performance is well within the specified requirement.

1.2.16 Process Instrumentation. The process instrumentation uncertainty includes those process instruments which will be recorded on the DAVDS along with the experiment instrumentation. These recorded process measurements are to be used to help supply additional data for the analysis of the LOFT expcriments.

The process instruments have several significant differences from the experiment instrumentation. The largest and probably the most significant of these differences is that the process measurements were designed to handle steady state conditions. The response time of a process measurement channel is typically $70 \mathrm{msec}$ or greater. Another major difference is that the process instrument is generally purchased as a module, while the transducer and electronic processor are procured as an integrated system. With this in mind, the uncertainty for a process measurement has been determined by the root sum square total of all the manufacturers' specifications. 


\section{Parameter}

Statistical error

Nonmono energetic $\gamma$

Stability

Calibration

Linearity

Noise
Uncertainty $( \pm \%)$

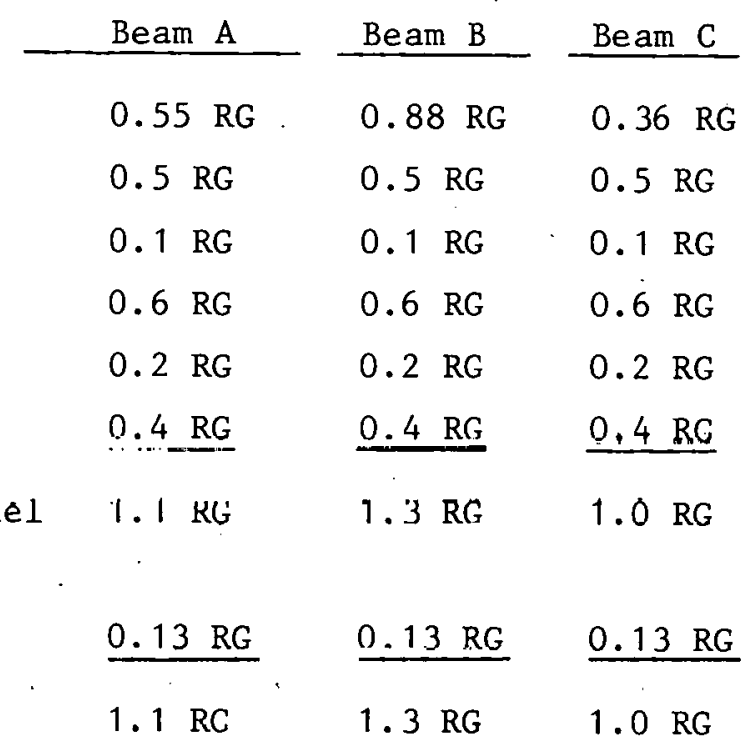

Response (10 to $90 \%$ rise time) is $35 \mathrm{msec}$.

This analysis is for steady state conditions. Most process instrumentation recorded on the DAVDS will be used only for steady state data. As additional data becomes available on the few dynamic process measurements, a more detailed analysis will be made. It has been assumed that the manufacturers' specifications have been normalized to the measurand "percent of range" and are statistically $3 \sigma$ values. If the $3 \sigma$ values are multiplied by $2 / 3$, an equivalent $2 \sigma$ uncertainty value can be obtained.

When the transducer/processor is connected to the DAVDS, a buffer amplifier is used between them.

\section{EXPERIMENTAL DETERMINATION OF LOFT PRIMARY COOLANT SYSTEM IIYDRAULIC PARAMETERS}

One of the objectives of functional lesting the LOFT plant prior to conducting loss-of-coolant experiments (LOCE) is to determine experimentally the hydraulic parame ters of the LOFT primary coolant system. The values of these parameters are used to check scaling criteria and LOCE predictions to ensure proper definition of specific LOCEs and acquisition of meaningful experimental data. The experimental determination of two such hydraulic variables, the emergency core coolant (ECC) cold wall delay and the steam generator flow resistance, is discussed in the following sections. 


\section{$2.1 \quad$ LOFT ECC Injection Delay}

(V. T. Berta)

A time delay will occur between the initiation of ECC flow and the appearance of ECC water in the lower plenum of a pressurized water reactor (PWR). The time delay is due in part to the distance between the ECC control valve and the lower plenum and in part to the effect of vaporizing ECC water and possibly delaying ECC flow. The LOFT nonnuclear LOCE series will include ECC injection into the cold leg piping of the primary loop. Accumulator A (ACC-A), one of two accumulators, will be the ECC water source.

An experiment designated the "cold wall delay" experiment was performed to determine that part of the ECC injection delay which could be attributed to the distance between the ECC valve and the lower plenum. ACC-A pressure was set at nominal conditions (600 psi) as established for the planned nonnuclear LOCEs. The primary coolant system was empty and at ambient temperature and pressure conditions. The ECC system was actuated and the flow measured and recorded as a function of time for a reference.

The cold wall delay experiment results are shown in Figure II-2. Experimental data were obtained from a liquid level detector placed in the lower part of the downcomer and from visual observations. The cold wall delay was determined to be $1.9 \pm 0.5$ seconds from the experimental data.

The liquid level elevation uncertainty is \pm 1.25 in. for each of seven conductivity probes that make up the detector. The timing uncertainty associated with each conductivity probe was determined from the recorded output from each probe. Of the seven conductivity probes in the detector, five gave useful data. The timing uncertainty is associated with the time at which each conductivity probe became submerged. The timing uncertainty for two probes was very small ( \pm 0.1 second). Two other probes had uncertainties of \pm 0.2 second, and one other probe had a relatively large uncertainty of \pm 0.6 second.

Visual observations were signalled by switch open-close operations which were recorded on tape along with the conductivity probe data. Visual data include the following three events:

(1) Water first sighted in reactor vessel

(2) Water level reaching the middle plate of the lower core supports

(3) Water level reaching the top plate of the lower core supports.

A timing uncertainty of \pm 0.4 second was assigned to each measurement to account for human reaction time for expected events.

Both sets of data are consistent. A value of $1.9 \pm 0.5$ seconds can readily be ascertained from the approximate reactor vessel fill curve as compared to the fill curve which assumes no delay and that all water enters the reactor vessel. The data also show that not all of the ACC-A water enters the vessel. Some water enters the pump region where it is 


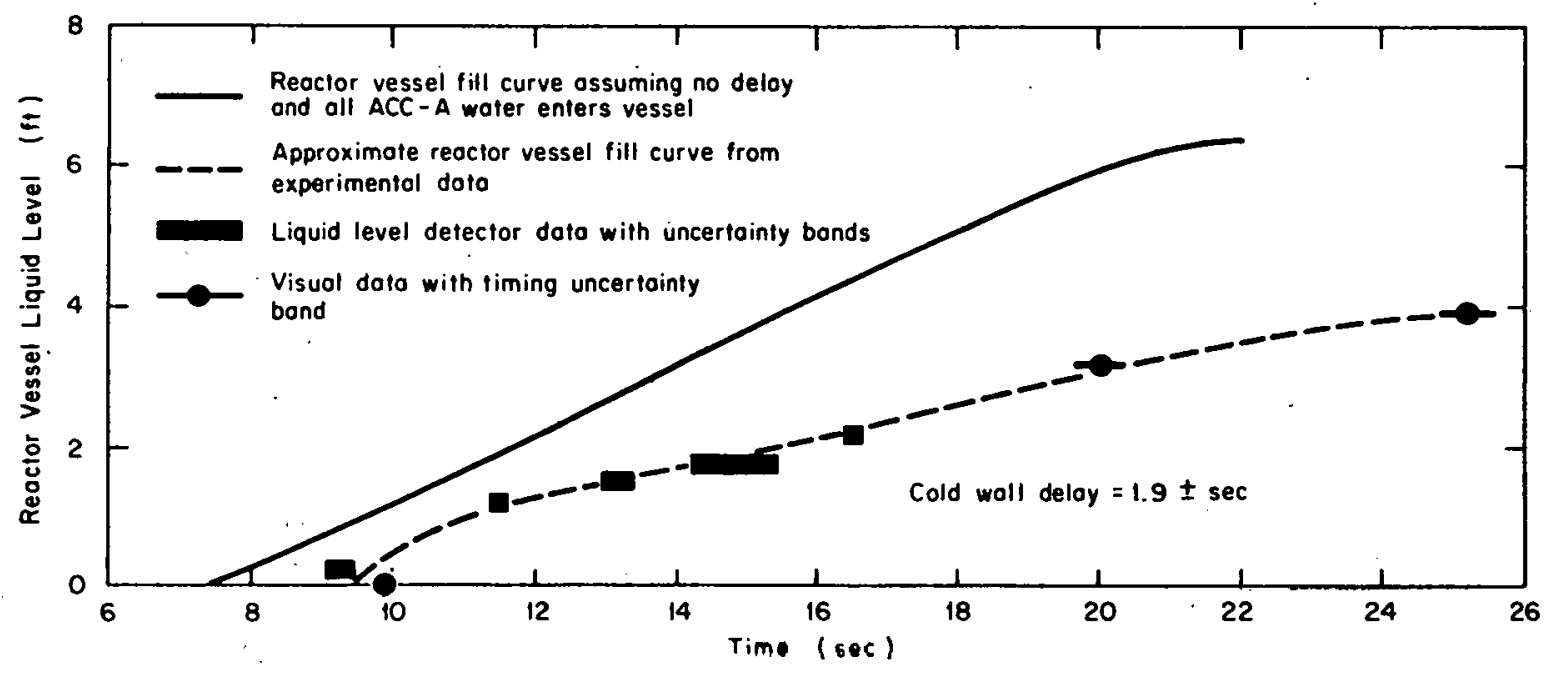

A. Accumulator A flow versus time.

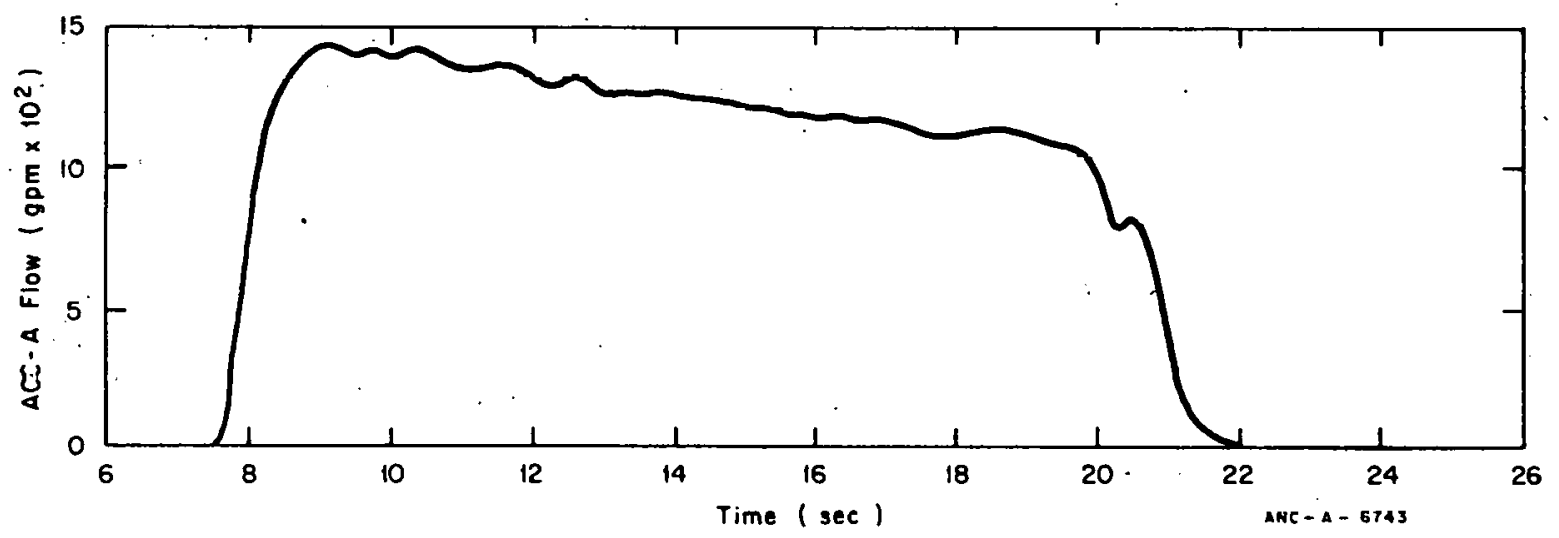

B. Reactor vessel liquid level versus time.

Fig. II-2 Cold wall delay experiment result - accumulator A to cold leg piping configuration.

trapped. Experimental data were not taken at higher reactor vessel liquid levels, so the amount of water that did not enter the reactor vessel cannot be determined exactly. However, that an estimated one-third of the available ACC-A water volume did not enter the vessel can be reasonably postulated based on the indicated trends toward the end of the fill transient.

The time at which water is first sighted in the reactor vessel is a few tenths of a second later than the time when water first reaches the lower plenum. Also, the conductivity probe at the bottom of the liquid level detector does indicate splashing effects, and thus records the presence of water slightly before the water reaches the lower plenum. The time of 1.9 seconds occurs at the overlap of the uncertainties of the measurements. The uncertainty, due to the bias, must be less than the extremes of the uncertainties in the measurements. The estimate of \pm 0.5 second for the uncertainty includes both measurements points but deletes the \pm 0.4 second for the reaction time uncertainty in the visual measurement. 
An approximate minimum possible value of the cold wall delay time can be calculated using the measured flow from the accumulator. The ECC injection nozzle into the cold leg piping has a flow area of $0.0645 \mathrm{ft}^{2}$ (4-in. Schedule 160 pipe). Using this, the injection velocity can be calculated as shown in Table II-XXV and in Equation (II-1). The ECC line is perpendicular to the primary loop pipe and, initially, flow can occur equally in both directions with complications due to splashing, water buildup, pipe friction, etc. For simplicity, conservation of energy will be used with the horizontal velocity equal to the vertical injection velocity over the $3.5 \mathrm{ft}$ of cold leg piping from the injection port to the inlet annulus.

TABLE II-XXV

COLD WALL DELAY TIME

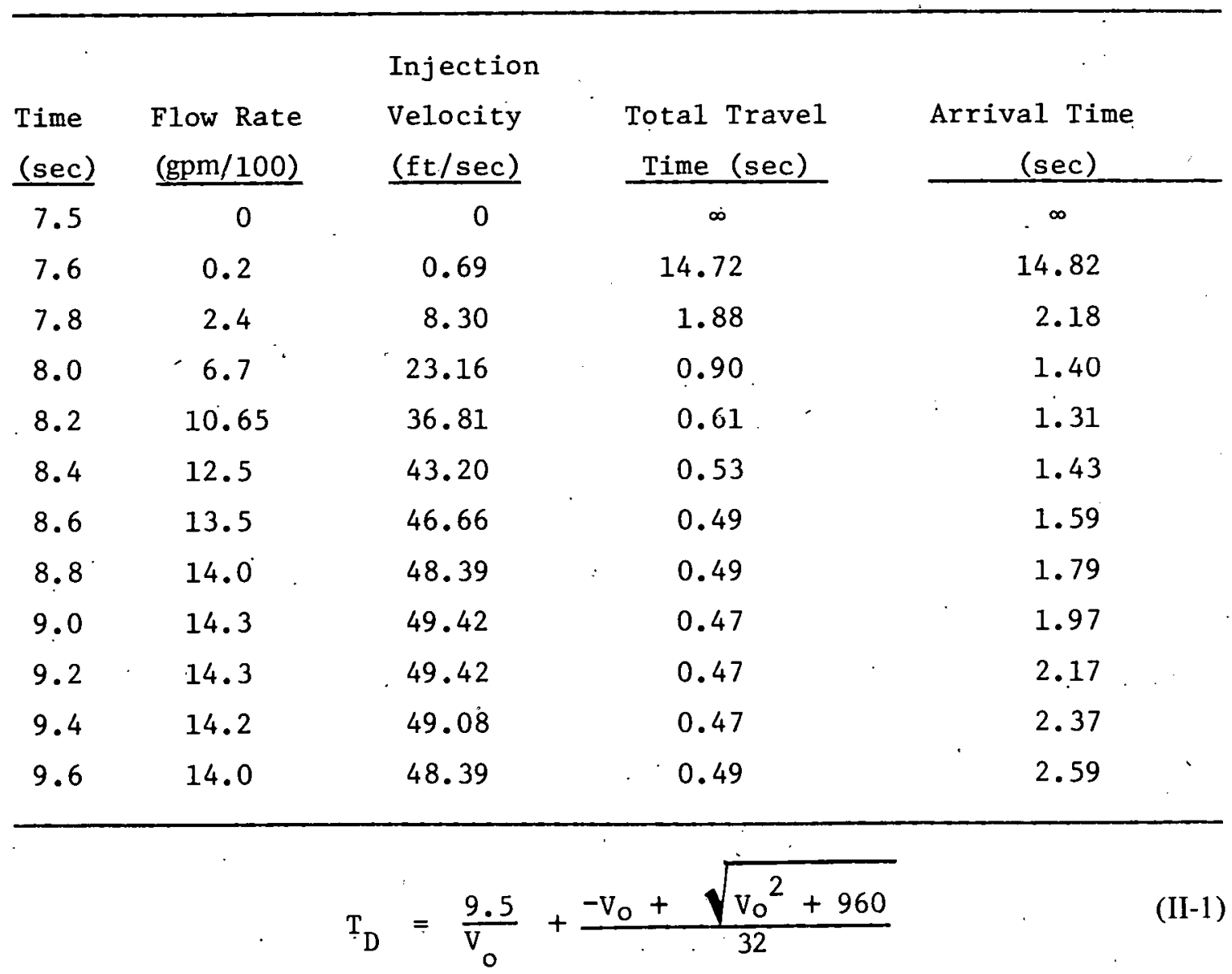

where

$$
\begin{aligned}
& \mathrm{T}_{\mathrm{D}}=\text { total travel time }=\frac{6+3.5}{\mathrm{~V}_{\mathrm{o}}}+\mathrm{T}_{3} \\
& \mathrm{~V}_{\mathrm{O}}=\text { injection velocity }=\frac{\mathrm{S}}{\mathrm{T}_{3}+1 / 2 \mathrm{~g} \mathrm{~T}} \sqrt{\mathrm{V}_{0}^{2}+4(15)(16)} \\
& \mathrm{T}_{3}=\text { time down the downcomer }=\frac{-\mathrm{V} \pm \sqrt{32}}{}
\end{aligned}
$$




$$
\begin{aligned}
& \mathrm{S}=\text { downcomer length }=15 \mathrm{ft} \\
& \mathrm{g}=\text { acceleration due to gravity }=32 \mathrm{ft} / \mathrm{sec}^{2} .
\end{aligned}
$$

At the junction of the primary pipe and the downcomer, the coolant is injected perpendicularly into a disk-shaped area that curves outward in the horizontal directions (looking into a vertical annulus horizontally from the side). Coolant can splash in a 360-degree circle; however, gravity will make the flow preferentially downward. Some coolant, due to the narrowness of the annulus, will experience only slight reduction in velocity and only a 90 -degree change in direction. The assumption is made, then, that the initial coolant velocity (disregarding mass) down the downcomer $\left(\mathrm{V}_{3}\right)$ is equal to the average coolant velocity in the primary pipe $\left(\mathrm{V}_{2}\right)$.

The total travel time from injection to the lower plenum can be found using the total path length prior to gravity acceleration in the downcomer $(6 \mathrm{ft}$ accumulator line plus $3.5 \mathrm{ft}$ cold leg pipe equal $9.5 \mathrm{ft}$ ) and the downcomer length $(15 \mathrm{ft})$ with gravity acccleration and the relationship shown in Equation II-1.

The arrival time for a given fluid particle is the total travel time plus the time after injection initiation that the given particle was injected. The minimum arrival time is the minimum cold wall delay time, namely, 1.4 seconds. The results of this analysis are shown in Table II-XXV and Figure II-3. The computed cold wall delay time is 1.3 seconds, which is slightly less than the measured value of 1.9 seconds, as one would expect. Based on this, the measured quantity $(1.9 \pm 0.5$ second) seems reasonable and will be used for comparison with the hot wall delay effect on ECC delivery, which will be experimentally determined in a subsequent LOCE.

\subsection{Steam Generator Flow Resistance \\ (R. K. Fujita)}

In order to properly scale the LOFT primary coolant system (PCS) flow resistance to the PCS flow resistance in a large pressurized water reactor, orifices will be placed in the inlet and outlet flanges of the LOFT steam generator. Prior to the first nonnuclear LOCE, designated $\mathrm{L} 1-1$, the predicted orifice flow resistances must be compared to experimental flow resistances.

The flow resistance of the steam generator in the intact operating loop was determined from experimental data obtained during the LOFT integrated plant acceptance tests. The pressure drop, mass flow rate, and fluid temperature were recorded for the unorificed steam generator during the primary system initial fill tests. Similar measurements were made during the first part of the plant hot functional tests with the "high-resistance" orifice plates installed in the steam generator.

The experimentally measured pressure drops across the steam generator include the pressure loss of the inlet and outlet piping. A schematic of the steam generator and its associated piping is shown in Figure II-4. An effective steam generator piping resistance coefficient $\left(\mathrm{K}_{\text {pipe }}\right)$ of 0.89 was computed and subtracted from the resistance coefficient 


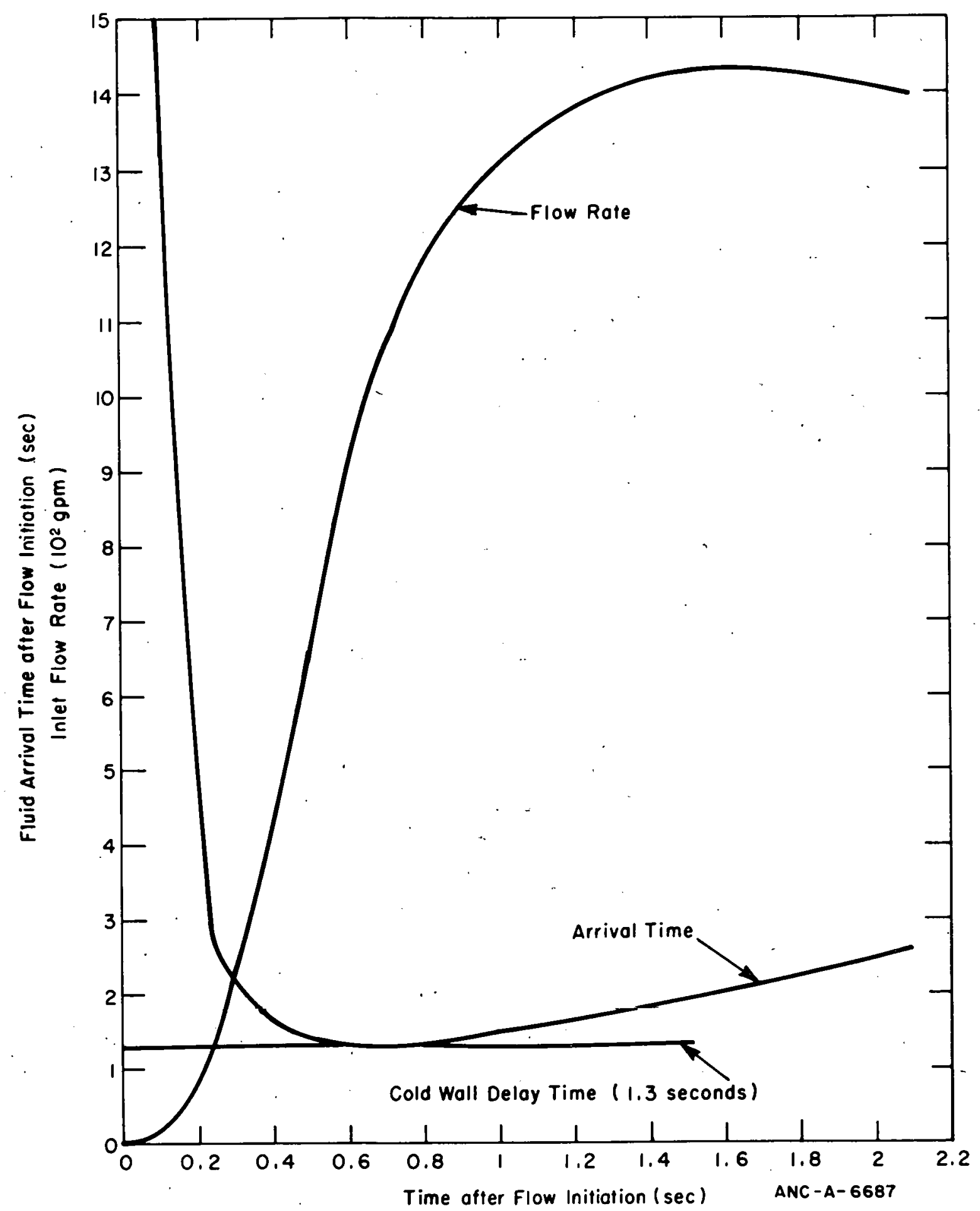

Fig. II-3 Calculated minimum cold wall delay.

determined from the experimentally measured steam generator pressure drops. A summary of the individual piping component resistances used to determine an effective piping resistance is presented in Table II-XXVI. 


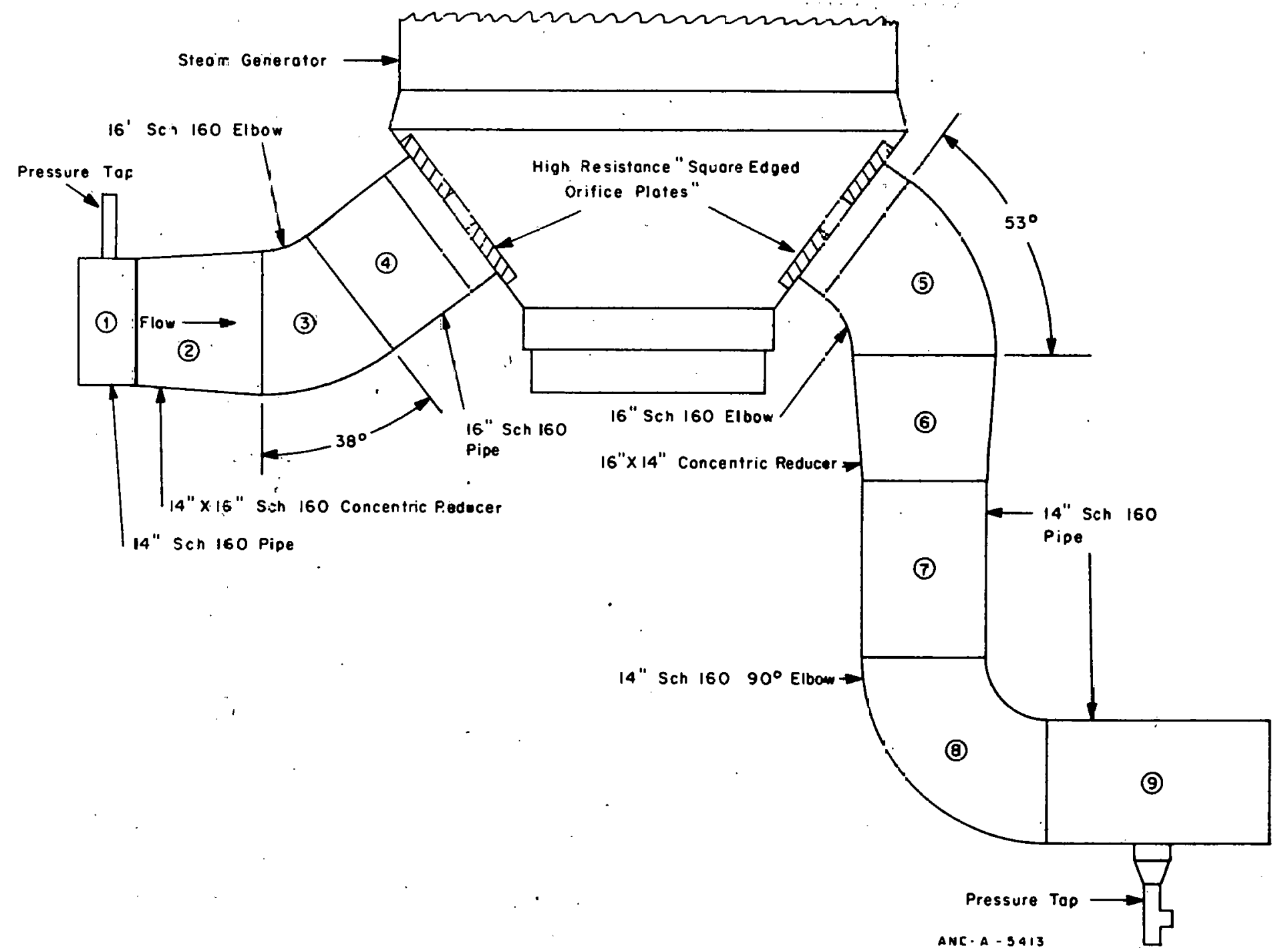

Fig. II 4 Schematic of the LOFT steam generator piping and high-resistance orifice plates. 
TABLE II-XXVI

EFFECTIVE STEAM GENERATOR PIPING RESISTANCE

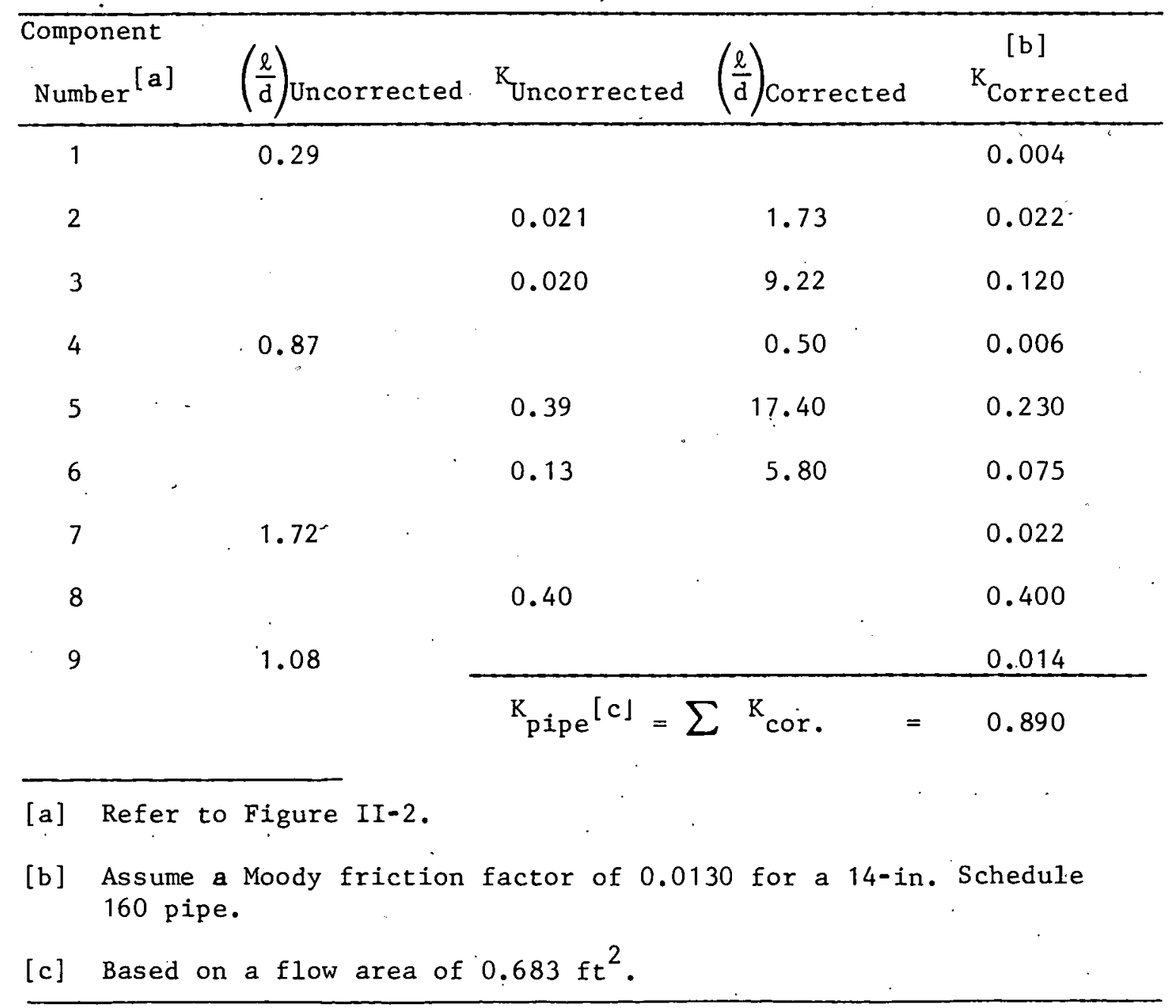

A mean, unorificed-steam-generator-resistance coefficient was calculated to be 2.65 with a $2 \sigma$ : standard deviation of \pm 0.16 (based on a 14-in. Schedule 160 pipe flow area of $0.683 \mathrm{ft}^{2}$ ). A predicted resistance for the unorificed steam generator is 2.25 . Therefore, the unorificed steam generator flow resistance is $15 \%$ greater than the predicted resistance.

The results of analyzing the data of the tests with the high-resistance orifice plates installed in the steam generator gave a mean flow resistance coefficient of 14.93 with a $2 \sigma$ of \pm 0.91 (based on a flow area of $0.683 \mathrm{ft}^{2}$ ). A resistance coefficient of 17.10 has been predicted which is $14.5 \%$ greater than the mean of the measured values. Consequently, the high-resistance orifice plates provide approximately $30 \%$ less flow resistance than they were originally designed to have. 
The calculational methods used for predicting the steam generator orifice size have been revised. The revised predictive method is based on the currently available experimental data from the LOFT acceptance tests. This method was used to size the "low-resistance". orifice plates which will be tested during Part 2 of the LOFT plant hot functional tests. The results of these tests will provide the information necessary to confirm the predictive method used to size the steam generator orifices.

\section{REFERENCES}

11-1. Aerojet 'Nuclear Company, Quarterly Technical Report on Water Reactor Safety Programs Sponsored by the Nuclear Regulatory Commission's Division of Reactor Safety Research, January - March.1975, ANCR-1254 (September 1975).

II-2. H. N. Norton, Handbook of Transducers for Electronic Measuring Systems, Englewood Cliffs, New Jersey: Prentice Hall, Inc.; 1969. 


\title{
III. THERMAL FUELS BEHAVIOR PROGRAM
}

\author{
J. G. Crocker, Program Manager
}

The series of power-cooling mismatch (PCM) tests, designated the PCM-20 Test Series, has been initiated to provide in-pile experimental data on the behavior of pressurized water reactor fuel rods under power-cooling-mismatch conditions. Tests are performed (a) to characterize the behavior of single unirradiated PWR fuel rods at normal operating power densities through the range of densities over which critical heat flux (CHF) is exceeded and (b) to determine the modes, mechanisms, and consequences of fuel cladding failure under various test conditons of overpower or undercooling.

The following sections describe (a) metallurgical test results from the PCM-20 Test 8-1 RS and (b) preliminary test results from the PCM-20 CHF Scoping Test.

In the PCM-20 Test 8-1 RS, a PWR-type fuel rod was placed in the Power Burst Facility (PBF) reactor in-pile tube and was subjected to increasing power densities through the range exceeding critical heat flux. The PCM-20 CHF Scoping Test involved similar cyclic testing of a PWR-type fuel rod to determine characteristic CHF occurrence parameters.

\section{PCM-20.TEST 8-1 RS, METALLURGICAL TEST RESULTS}

R. R. Hobbins and G. W. Gibson.

\subsection{Introduction}

The objectives of the PCM-20 Test 8-1 RS were outlined in an earlier quarterly report for January - March 1975, ANCR-1254[III-1], and presented in detail in a subsequent report [III-2].

For the PCM-20 Test 8-1 RS the coolant mass flux, inlet temperature, and pressure. were $1 \times 10^{6} \mathrm{lb} / \mathrm{hr}-\mathrm{ft}^{2}, 622^{\circ} \mathrm{F}$, and 2,080 psig respectively. CHF first occurred at a peak fuel rod power of $15.9 \mathrm{~kW} / \mathrm{ft}$. Cladding surface temperature oscillations were observed for fuel rod powers from $15.9 \mathrm{~kW} / \mathrm{ft}$ to $24.3 \mathrm{~kW} / \mathrm{ft}$. Sustained film boiling was achieved during two of the four power increases performed. The test fuel rod cladding failed following reactor shutdown. The postirradiation examinations (PIE) of the fuel rod have been completed and the results of this work are discussed below.

\subsection{Visual Examination}

When the fuel rod was removed from the experiment shroud, it was discovered that the rod was in two pieces with the separation at 24-1/4 inches from the bottom of the rod. As the lower portion of the rod was lowered onto a table, the broken end of the rod 
touched the table first and broke from the weight of the rod (the break occurring 21-1/4 inches from the bottom of the rod. Both fractures occurred through the main portion of a fuel pellet, and not between a pellet-to-pellet interface. The fractures appeared to be brittle with no evidence of ductile tearing. The cladding showed areas of heavy oxidation and pronounced collapse between pellets. Because of the brittleness of the rod, neutron radiography and gamma scanning were not performed, in order to avoid further damage to the rod during handling.

\subsection{Destructive Examination}

Great care was taken in preparing the fuel rod for sectioning to assure that no damage was done to the fragile materials. Details of the locations of the samples removed for examination and preparative methods will be given in the test results report which is currently being prepared. Highlights of the results of these examinations are described belnw.

1.3.1 Cladding Examination. Metallographic specimens were etched $\left(5 \mathrm{~m} 1 \mathrm{H}_{2} \mathrm{O}, 5 \mathrm{ml}\right.$ $\mathrm{HNO}^{3}, 2 \mathrm{ml} \mathrm{HF}, 15 \mathrm{ml}$ lactic acid) and the microstructure in the zircaloy cladding was examined.

Structures varied from essentially undisturbed as-received (stress-relieved) material (Figure III-1); to recrystallized material (Figure III-2); to material showing prior beta phase $(\beta-\mathrm{Zr})$, oxygen-stabilized alpha phase $(\alpha-\mathrm{Zr})$, and $\mathrm{ZrO}_{2}$ (Figure III-3); to heavily oxidized material having almost all of the wall thickness taken up by $\mathrm{ZrO}_{2}$ and oxygen-stabilized alpha phase (Figure III-4).

Oxygen-stabilized alpha layers of roughly the same thickness were observed on both the inside and outside surfaces of the cladding. The oxygen-stabilized alpha layer on the inside surface of the cladding is most likely the result of $\mathrm{UO}_{2}-\mathrm{Zr}$ reaction. 'I'he observed disappearance of this layer in the cladding adjacent to the gaps between pellets (Figure III-5) is consistent with this mechanism. However, the lack of a metallographically observable interaction layer and the inability to detect a uranium-rich layer between the $\mathrm{UO}_{2}$ and zircaloy by electron microprobe are inconsistent with previous reports of this reaction [III-3, $111-4]$.

The cracks observed in the cladding (Figures III-4, III-6, and III-7) could have admitted steam which could then have reacted with zircaloy to produce the oxygenstabilized alpha layer on the inner surface of the cladding. However, this mechanism would require the presence of the steam for essentially the entire duration of the high temperatures, whereas the internal pressure indication of a cladding failure occurred only on the final cooldown.

Although the lack of fuel-cladding contact at gaps between pellets undoubtedly results in some localized cooling of the interior surface of the cladding, the prior beta structure in Figure III-4 suggests that the cladding had not cooled sufficiently to prevent the formation of an oxygen-stabilized alpha layer in the presence of steam. 
75-P-110

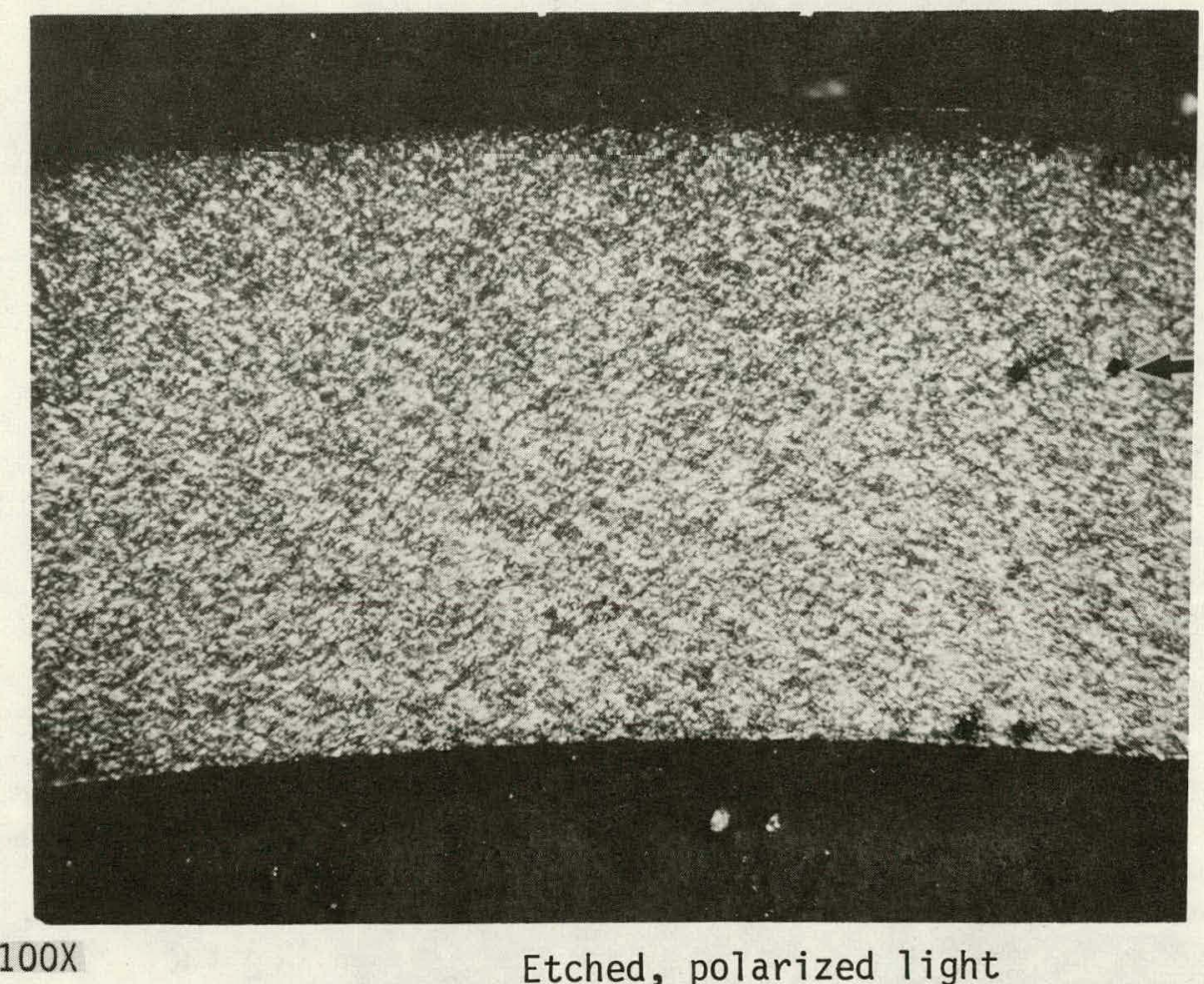

Microhardness indention in cladding

Fig. III-1 Stress-relieved structure in zircaloy cladding at 8-1/4 inches from bottom of fuel rod (PCM-20 Test 8-1 RS).

\section{5-P-78}

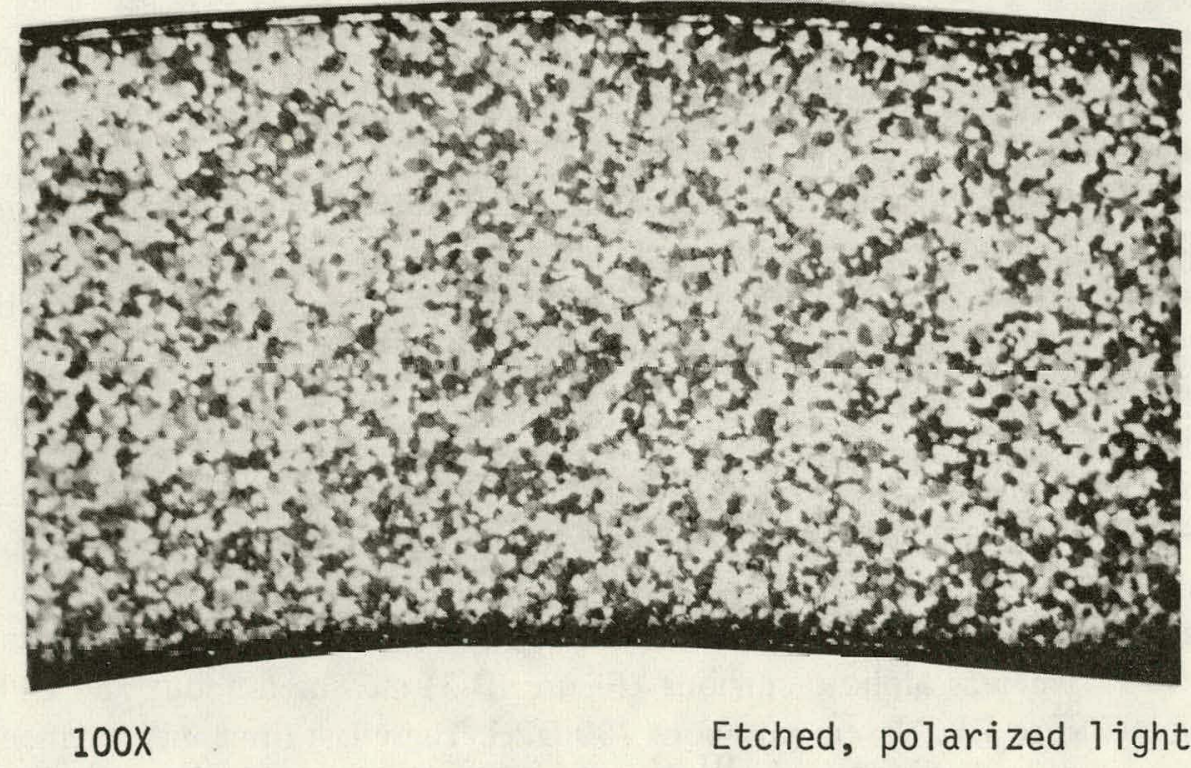

Fig. III-2 Recrystallized structure in zircaloy cladding, 31-7/8 inches from bottom of fuel rod (PCM-20 Test 8-1RS). 


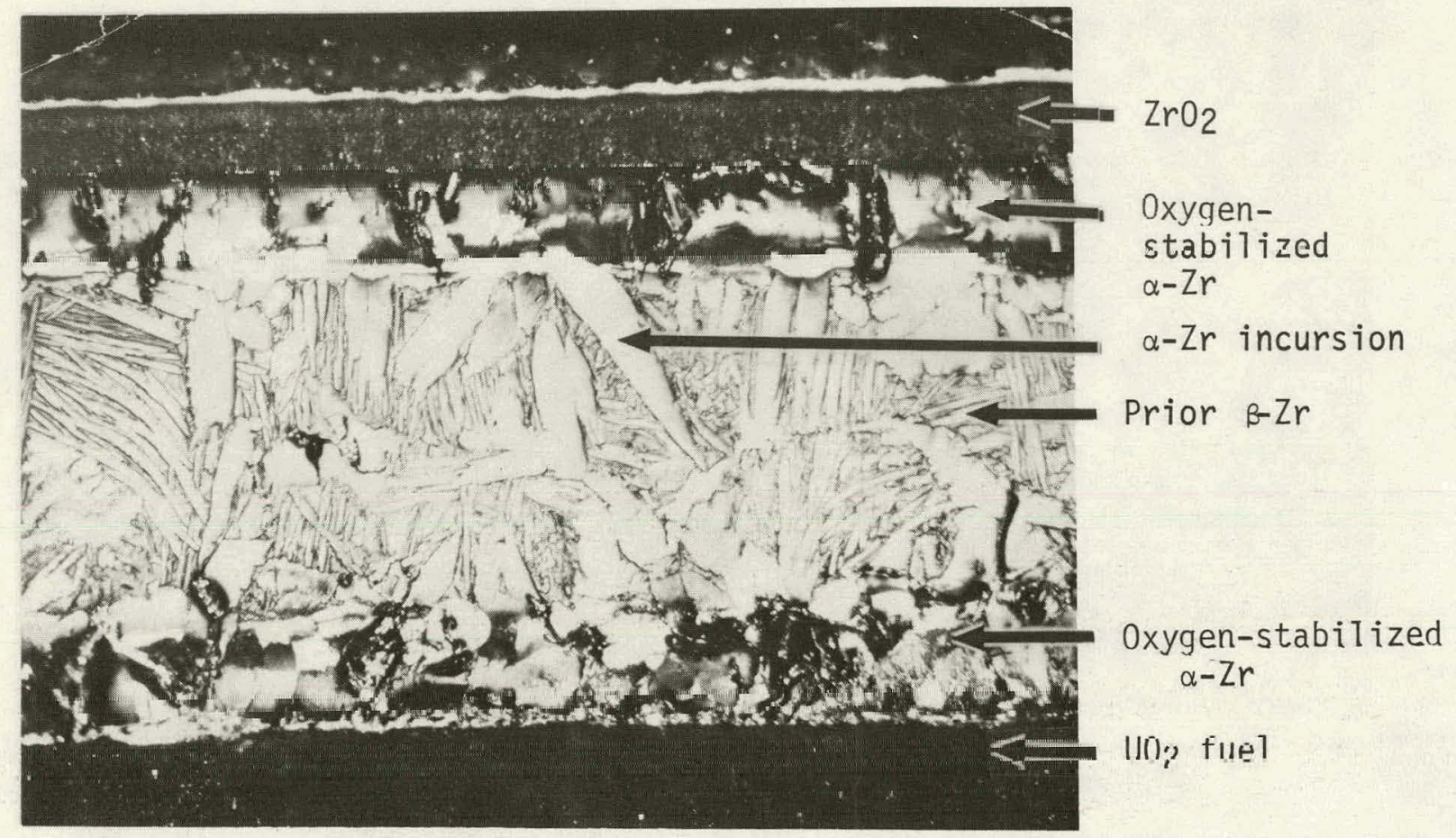

$100 x$

Etched, polarized light

Fig. III-3 A longitudinal section of fuel rod at about 23 inches from bottom of rod showing prior $\beta$-Zr, oxygen-stabilized $\alpha-\mathrm{Zr}, \alpha-\mathrm{Zr}$ incursions, and $\mathrm{ZrO}_{2}$ in cladding structure (PCM-20 Test 8-1 RS).

The final resolution of the cause of the oxygen-stabilized alpha layer on the inside surface of the cladding may come from subsequent tests to be conducted in PBF in which fuel rods will be subjected to cladding temperatures sufficiently high to cause considerable oxidation but of short enough duration to allow retention of limited ductility to ensure rod integrity.

Microhardness measurements were taken across the cladding using a diamond indentor and a 100-gram load. The microhardness in the undisturbed stress relieved material (Figure III-1) was 232 DPH (diamond pyramid hardness). The annealed material (Figure III-2) was relatively soft, measuring $191 \mathrm{DPH}$. Hardness of about $350 \mathrm{DPH}$ was measured in prior beta material (Figure III-3), whereas alpha incursions (Figure III-3) measured about 550 DPH, and the alpha layers (Figure III-3) averaged about $750 \mathrm{DPH}$. These last three measurements are higher than those reported by Hobson [III-6] $(330 \mathrm{DPH}, 455 \mathrm{DPH}$, and $536 \mathrm{DPH}$ for prior beta structure, alpha incursions, and alpha layers, respectively) in zircaloy oxidized in single-cycle isothermal tests.

Cladding temperatures were estimated by two methods: (a) by relating temperature and the combined thickness of the $\mathrm{ZrO}_{2}$ and alpha layers on the outside diameter of the 


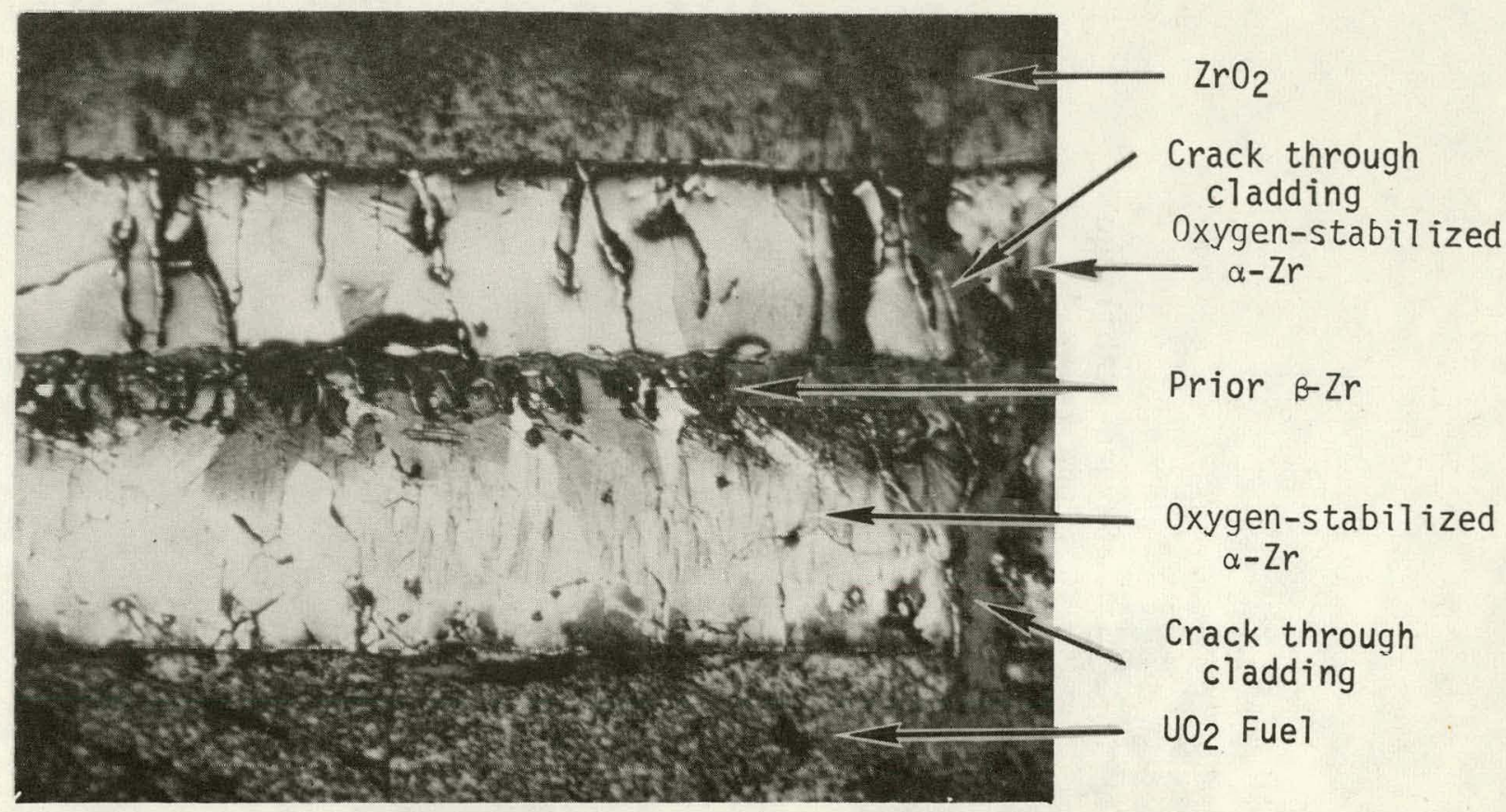

A. $100 X$

Etched, bright field

$75-P-145$

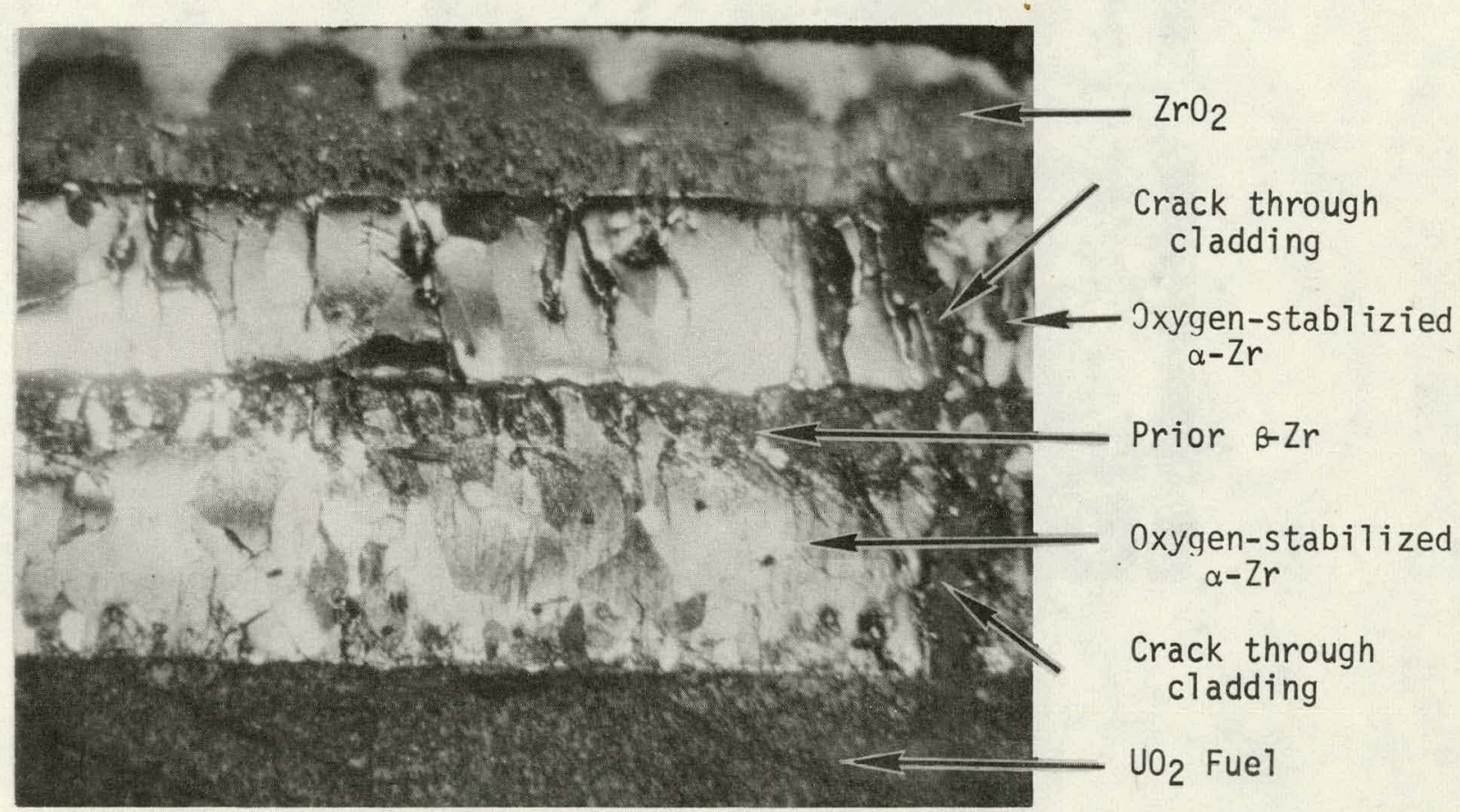

B. $100 x$

Etched, polarized light

Fig. III-4 Longitudinal section of fuel rod 24-1/2 inches from bottom of rod showing heavily oxidized cladding, with oxygen-stabilized $\alpha-\mathrm{Zr}$ taking up most of cladding wall thickness. Note crack through cladding (PCM-20 Test 8-1 RS). 
$75 P-10<$

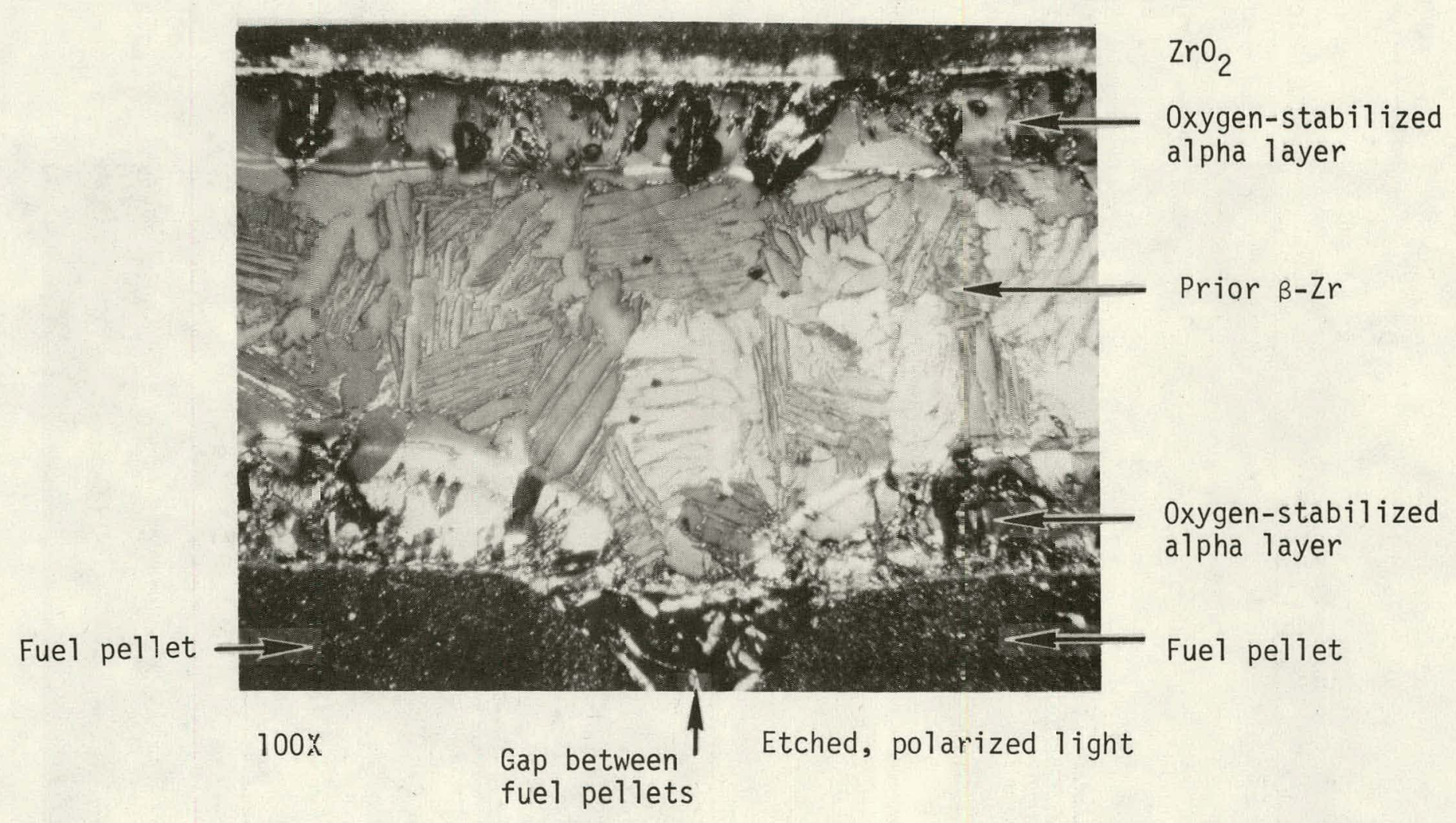

Fig. III-5 Lcngitudinal section of fuel rod at about 23 inches from bottom of rod showing absence of oxygen-stabilized alpha layer on inner surface of cladding adjacent to gap between fuel pellets (PCM-20 Test 8-1 R.S). 


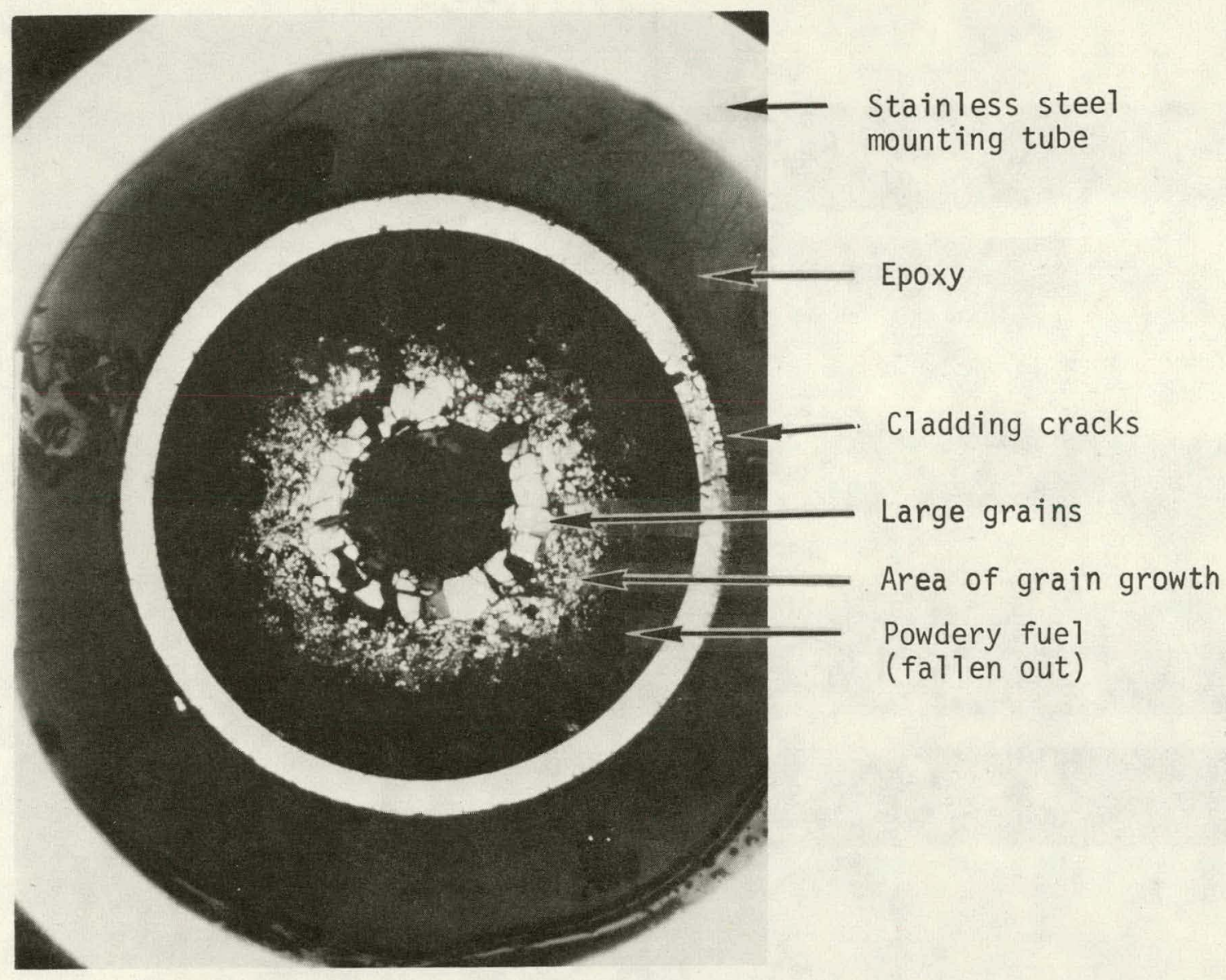

About $7 X$ Cladding etched, bright field

Fig. III-6 Transverse section of fuel rod showing fuel restructuring and cladding cracks at 24 inches from bottom of rod (PCM-20 Test 8-1 RS).

cladding and (b) by the microstructures exhibited in the cladding. In the first method, cladding temperatures were estimated based on a correlation which relates the sum of the thickness of the $\mathrm{ZrO}_{2}$ layer and the oxygen-stabilized layer on the cladding OD with the time and temperature of exposure, as established by the data of Meservey and Herzel [III-5] and Hobson and Rittenhouse ${ }^{[\mathrm{III}-6]}$. This method was found to calculate temperatures in reasonable agreement with those estimated from microstructures. The microstructure temperature estimates were made using structural characteristics such as the relative amounts of alpha phase and prior beta phase $\mathrm{Zr}$, prior beta grain size, the coarseness of the alpha transformation structure, and the amount and distribution of oxygen-stabilized alpha phase $\mathrm{Zr}$.

Both approaches were used to generate the temperature diagram shown in Figure III-8. The maximum temperature in each metallographic sample was usually near the location of the 25-inch thermocouple, and the minimum temperature was on the opposite 


\section{Collapse of cladding between fuel pellets}

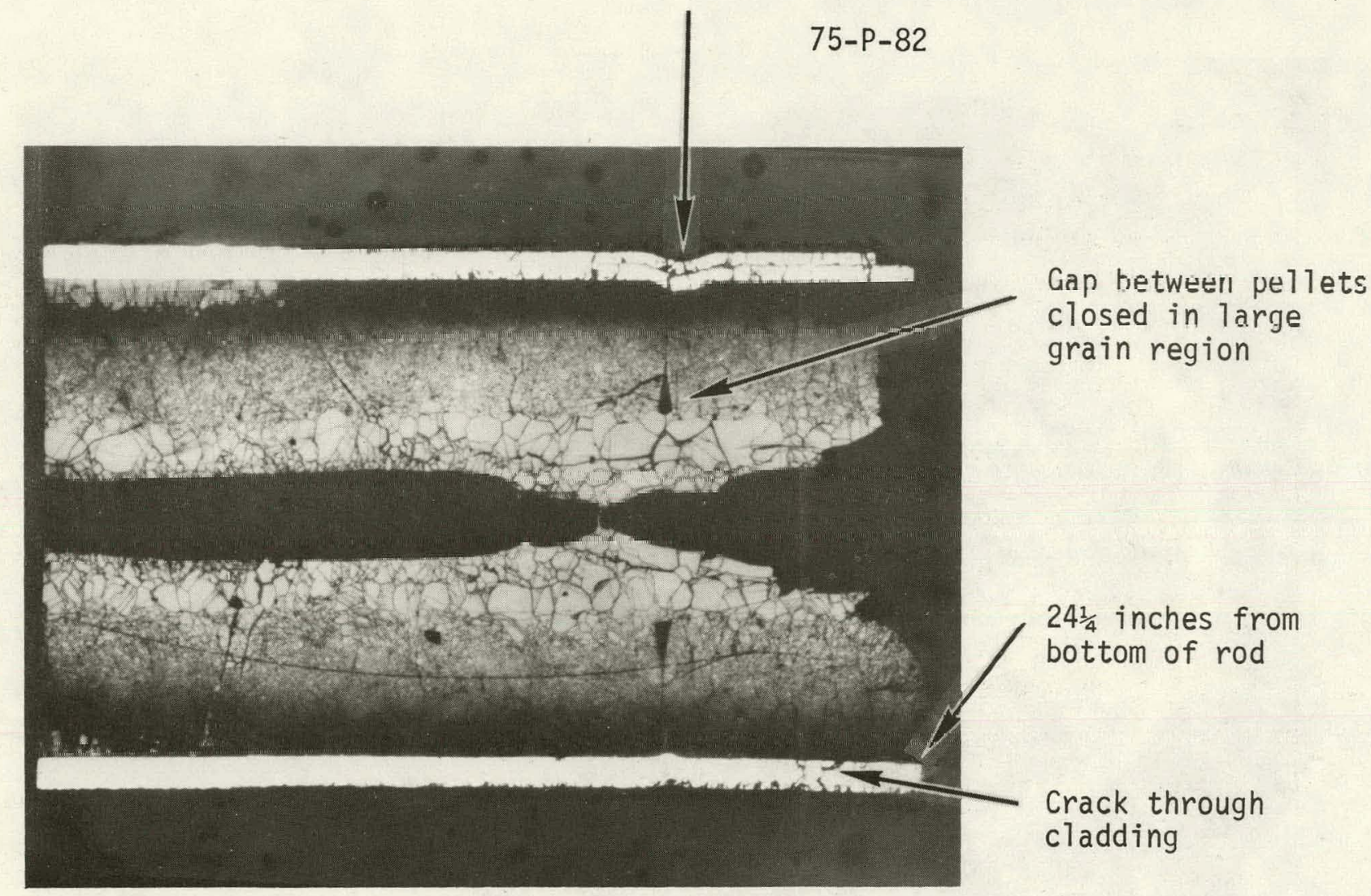

About $7 X$

Cladding etched, bright field

Fig. III-7 Longitudinal section of fuel rod showing fuel restructuring, cladding collapse, and cracks through cladding from 24-1/4 to 25 inches from bottom of fuel rod (PCM-20 Test 8-1 RS).

side of the fuel rod near the location of the 27 -inch thermocouple. This usual pattern was strongly disturbed, however, at the junction of the 25 -inch thermocouple as is illustrated in Figure III-9. It is apparent that the cladding structure was considerably cooler beneath the 25 -inch thermocouple than it is to either side. The prior beta structure vanishes in the weld between the cladding and the thermocouple sheath, and the sheath itself shows no evidence of having been at a temperature greater than about $1,400^{\circ}$ to $1,500^{\circ} \mathrm{F}$ which is in good agreement with the $1,530^{\circ} \mathrm{F}$ measurement indicated by this thermocouple: Not far from the 25 -inch thermocouple, however, cladding temperatures of about $2,200^{\circ} \mathrm{F}$ were reached. This strong temperature gradient indicates that the thermocouple sheath acted as a cooling fin to remove heat lium the cladding. Thus, the cladding surface thermocouples were probably indicating thermocouple sheath temperatures quite accurately, but the cladding surface temperatures were much larger than the thermocouple sheath temperatures.

Figure III-10 shows the results of fuel rod cladding collapse at relatively low temperatures. As shown in this figure, a section of cladding material has collapsed into a void left by the chipped end of a fuel pellet. The deformation occurred prior to the absorption of significant quantities of oxygen, indicated by the lack of microcracks and a high degree of ductility. The probable temperature of this phenomenon is between 1,470 


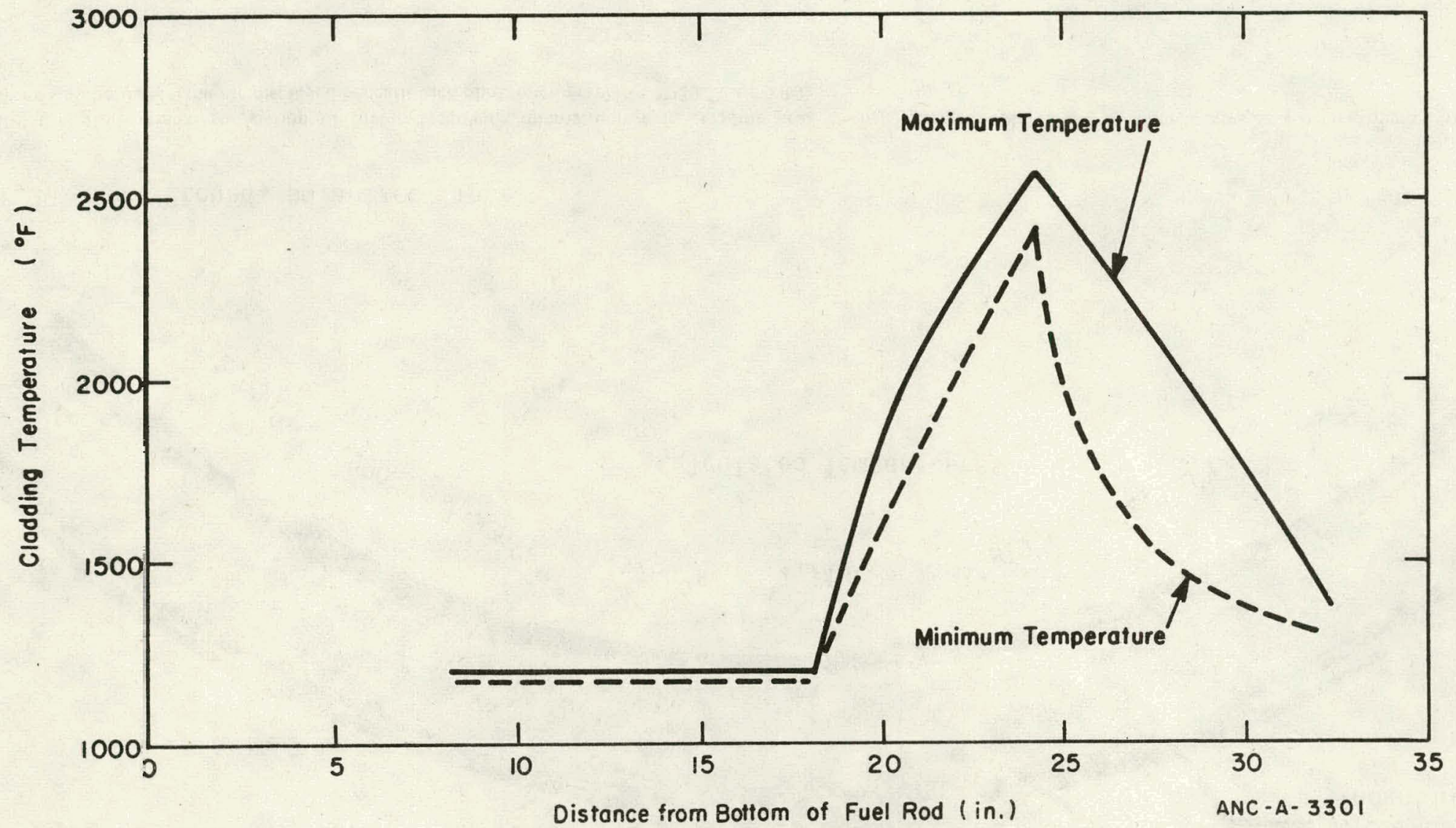

Fig. III-8 Cladding temperature axial profile estimated from metallurgical examination of the PCM-20 Test 8-1RS. 


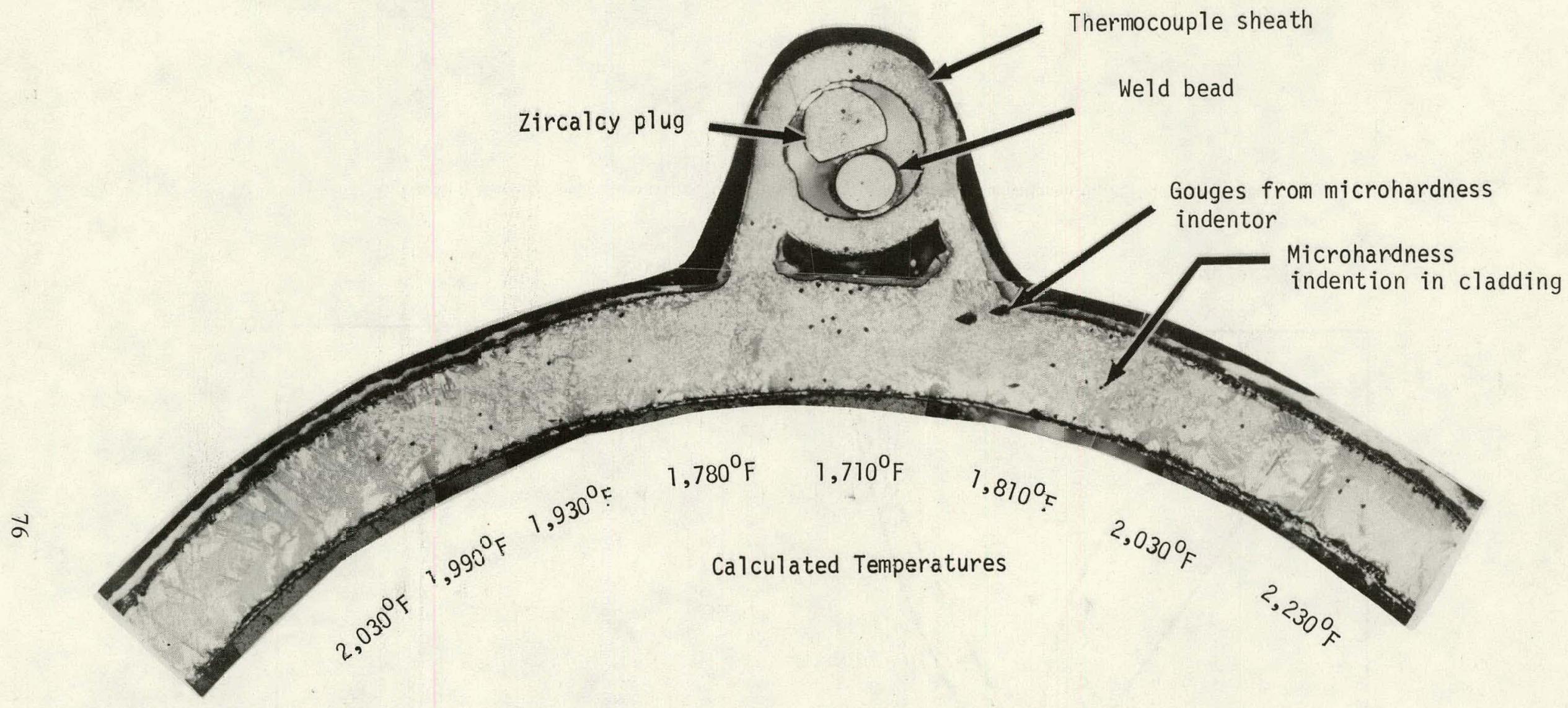

About 30X Etched, polarized light

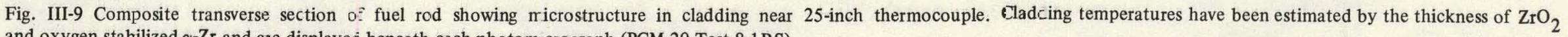
and oxygen stabilized $\alpha-\mathrm{Zr}$ and are displayec beneath exch photom.crograph (PCM-20 Test 8-1RS). 


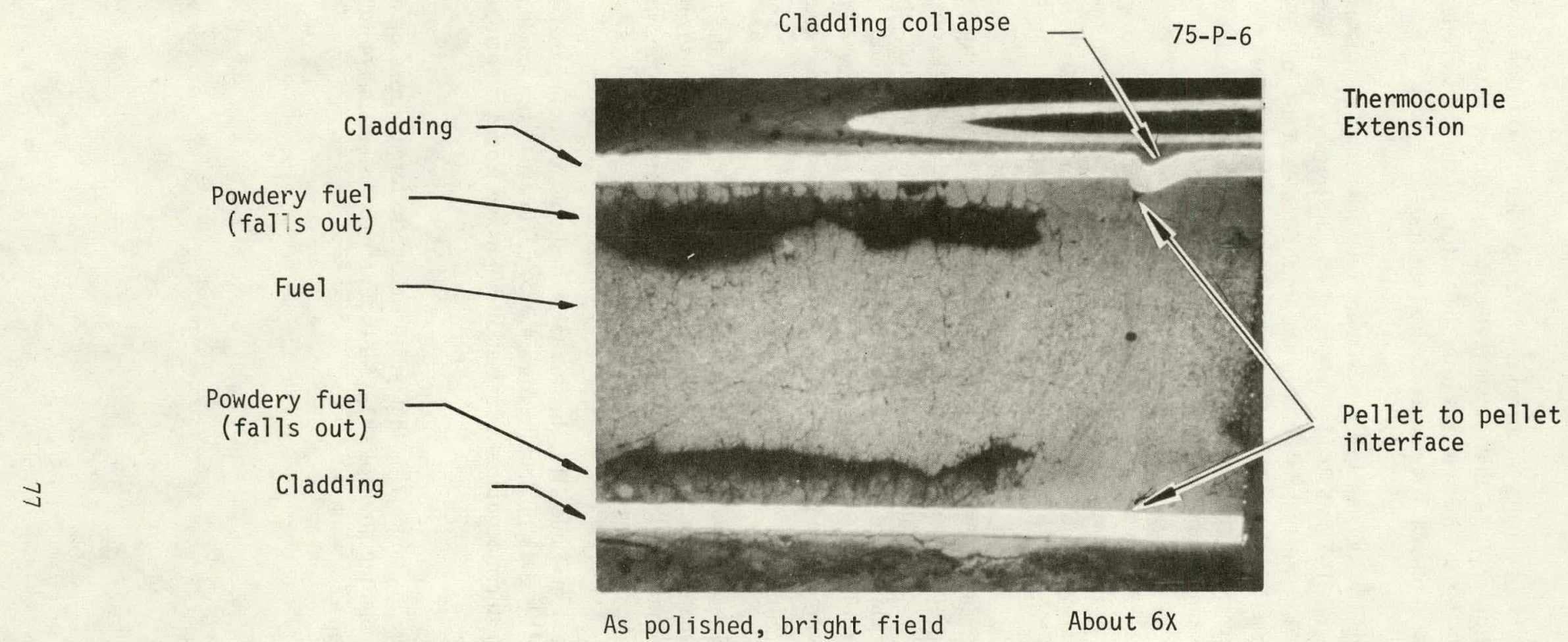

Fig. III-10 Longitudinal section of fuel rod taken at about 27 inches from bottom of rod. Note that a portion of the cladding has collapsed into a void between two fuel pellets (PCM-20 Test 8-1 RS). 
and $1,560^{\circ} \mathrm{F}^{[\mathrm{III}-7]}$. Thus, cladding collapse occurred prior to significant oxidation. Visual and metallographic examination revealed that substantial cladding collapse between fuel pellets occurred from about 24-3/8 to 28-3/4 inches from the bottom of the rod while lesser indications of cladding collapse extended from about 20 to 31 inches.

1.3.2 Fuel Examination. The $\mathrm{UO}_{2}$ fuel shows evidence of considerable restructuring as illustrated in Figures III-6 and III-7 and III-11 through III-15. The restructuring consists of grain growth which increases up the thermal gradient toward the center of the fuel pellet. The restructuring is also characterized by shattering of the fuel along boundaries in large grains near the center of the pellet (Figures III-13 and III-15). The healing of the gap between pellets in the central zone of large, dense grains is evident in Figure III-7.

X-ray diffraction studies were made on a fuel rod section similar to that shown in Figure III-11. The purpose of this work was to identify the cause of the variation in microstructure in the $\mathrm{UO}_{2}$ around the centerline hole. No change in the $\mathrm{UO}_{2}$ lattice parameters was detected. Likewise, no foreign elements could be identified.

Fuel temperatures were calculated from the grain sizes measured by the intercept method and grain growth kinetic equations presented by Ainscough et al ${ }^{[\mathrm{III}-8]}$, Lyons et al [III-9]; and Nichols[III-10]. These methods are unlikely to be reliable without having the out-of-pile grain growth kinetics of the particular $\mathrm{UO}_{2}$ fuel used. Furthermore, the equations were developed for steady state conditions while the test involved fast temperature transients. The temperature dependence of the grain size for the three kinetic models is shown in Figure III-16.

The initial grain size $(3.4 \mu \mathrm{m})$ was determined from Figure III-17, which is the fuel structure near the outside diameter of the fuel at 30-1/4 inches from the bottom of the rod. Grain sizes were measured near the center of the fuel where equiaxed grains of consistent size were found.

The results are presented in Table III-I. In this table it should be noted that the correlation from Nichols $[111-10]$ gives the temperature, $4,075^{\circ} \mathrm{F}$, closest to the maximum indicated by the fuel centerline thermocouple, $4,155^{\circ} \mathrm{F}$ at $30-1 / 4$ inches from the bottom of the rod.

It should also be noted in Table III-I that all three kinetic formulations predict temperatures in excess of the melting point of $\mathrm{UO}_{2}, 5,140^{\circ} \mathrm{F}$, for the fuel at $24-1 / 4$ inches from the bottom of the fuel rod. 


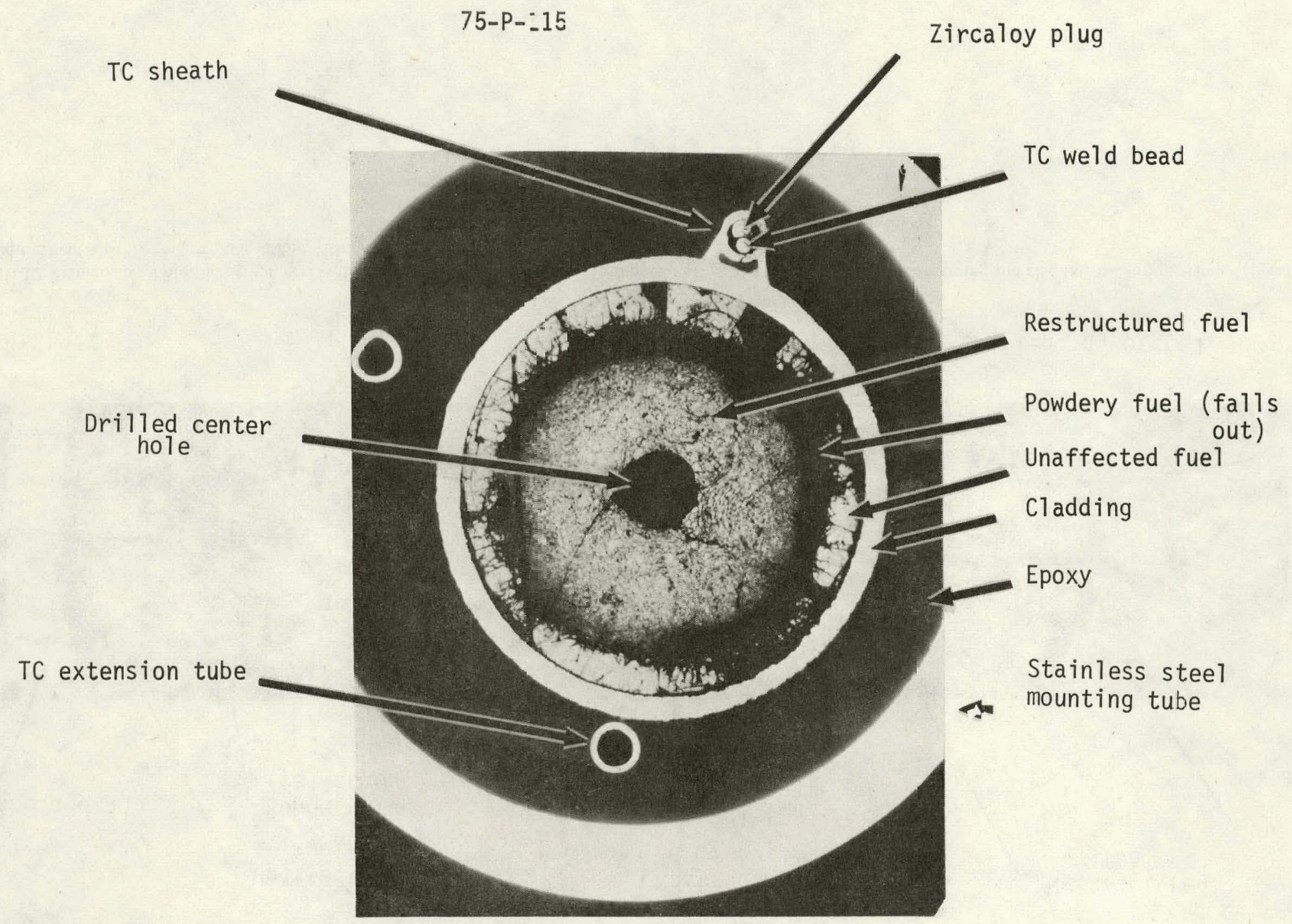

About $7 X$

Cladding $\in$ tched, bright field

Fig. III-11 Transverse section of fuel rod $25-7 / 8$ inches from bottom of fuel rod. View is from bottom of rod. (PCM-20 Test 8-1RS) 


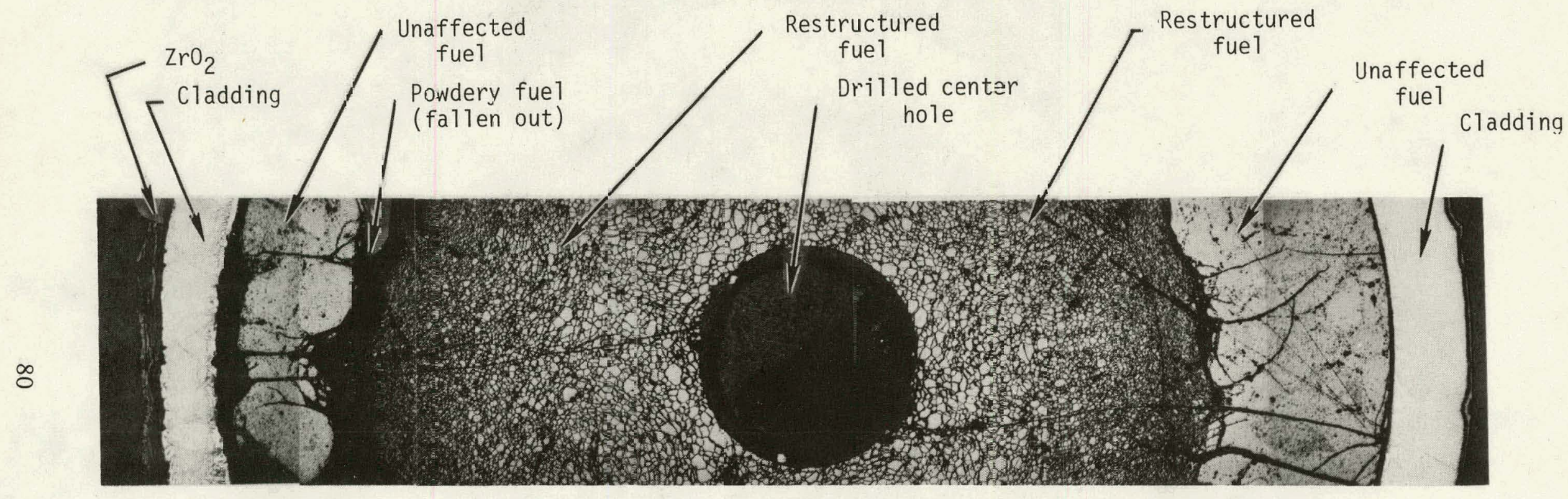

About $20 \times$

Cladding etched, bright field

Fig. III-12 Composite photoricrcgraph ccross fuel rod at about 27-3/4 inches from bottom of fuel rod showing full restracturing cnd cladding oxidation. The UO 2 grain size increases toward the center of the pellet anc is visitle without etching the fuel (PCM-20 Test 8-1RS). 


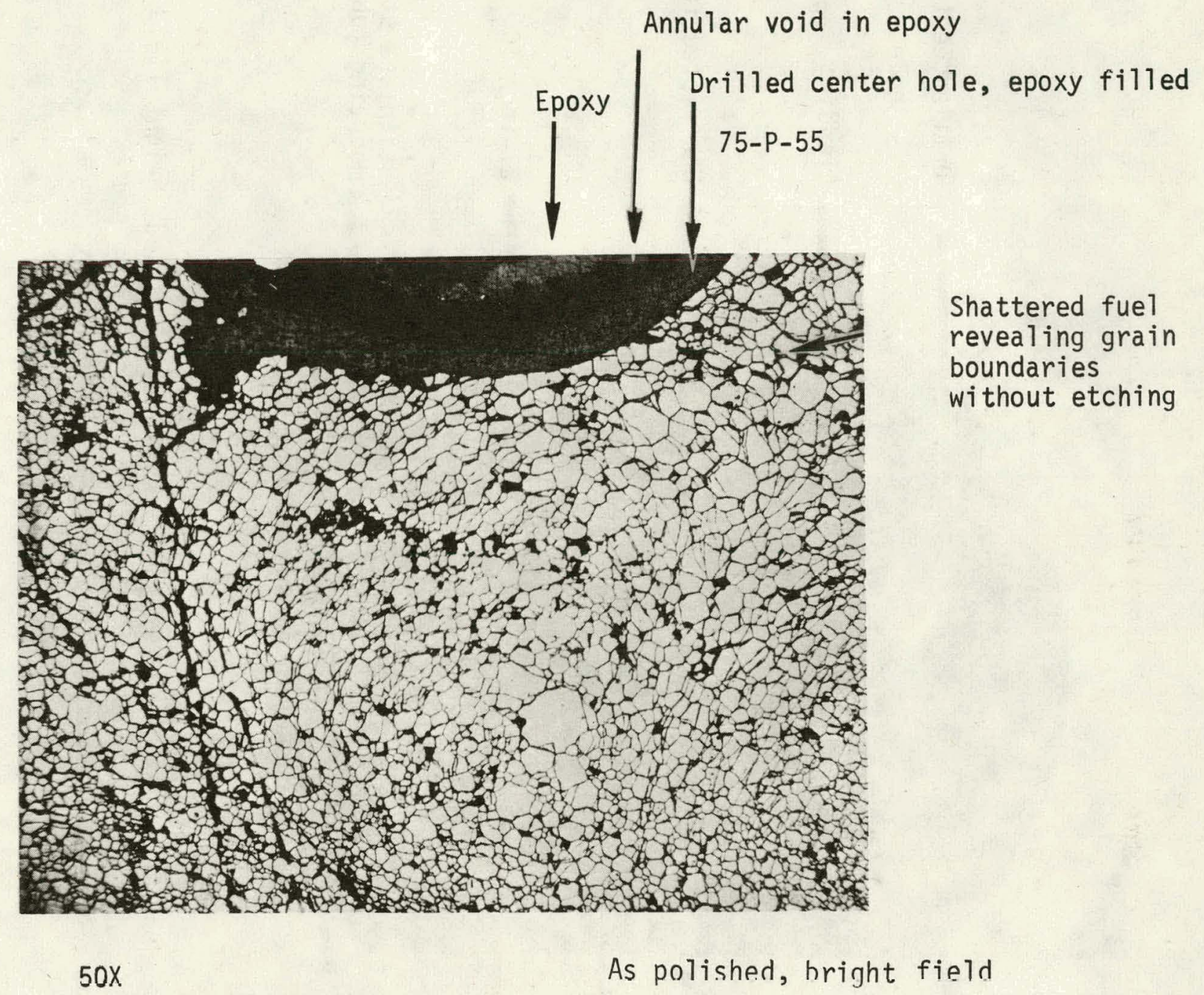

Fig. III-13 Transverse section of fuel rod showing fuel structure near center hole of fuel pellet, 27-3/4 inches from bottom of rod (PCM-20 Test 8-1RS). 


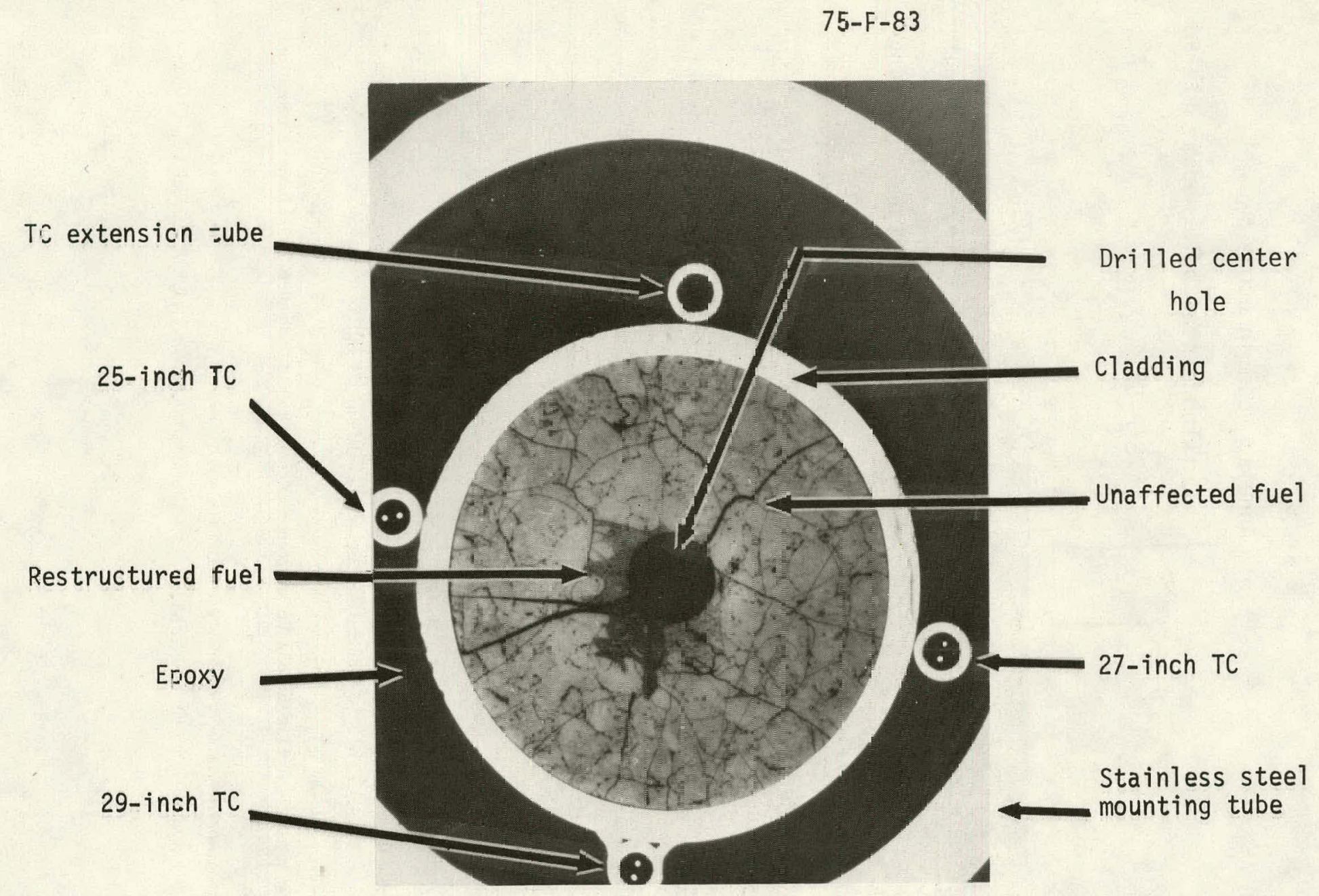

About $7 X$

C- adding etched, bright field

Fig. II-14 Transverse section of fuel rod 30-1/4 inches from bot:on of rod. View is from top of rod (PCM-20 Test 8-1 RS). 


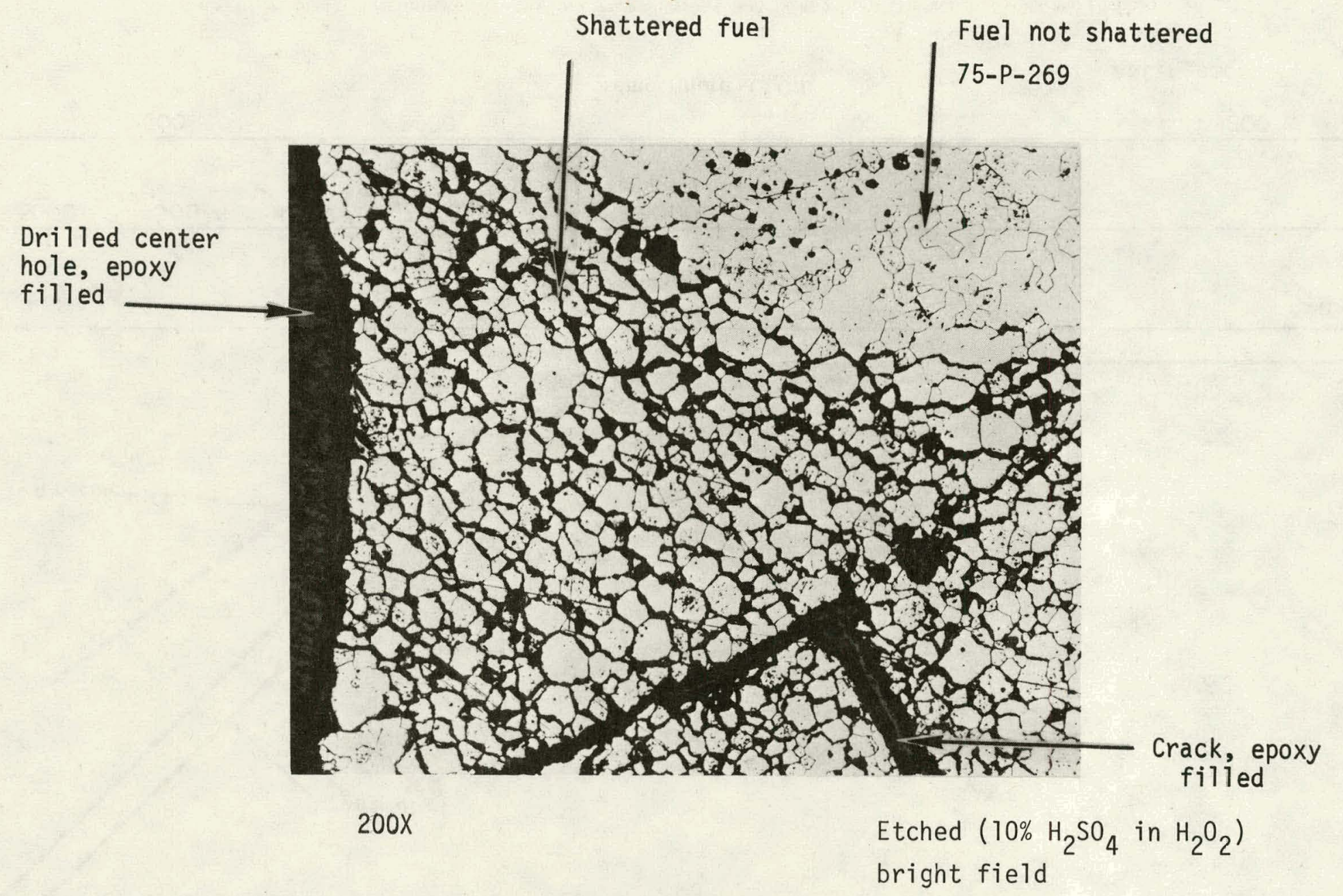

Fig. III-15 Transverse section of fuel rod showing fuel structure near center hole of fuel pellet at 30-1/4 inches from bottom of rod (PCM-20 Test 8-1 RS). 


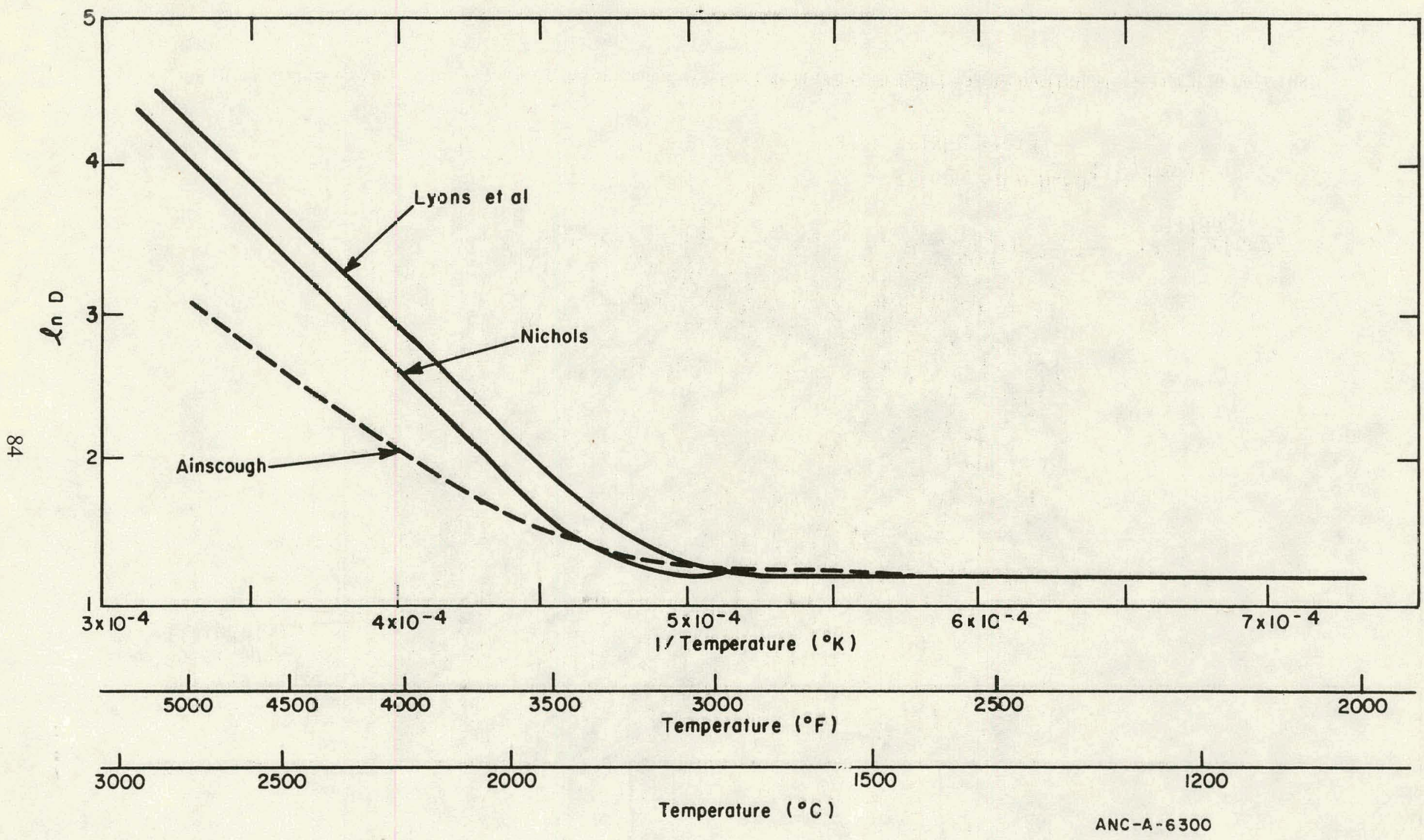

Fig. III-16 Fuel temperature vs. grain size for three grain growth kinetic equations (Ainscoush. Lyons, and Nichols). 


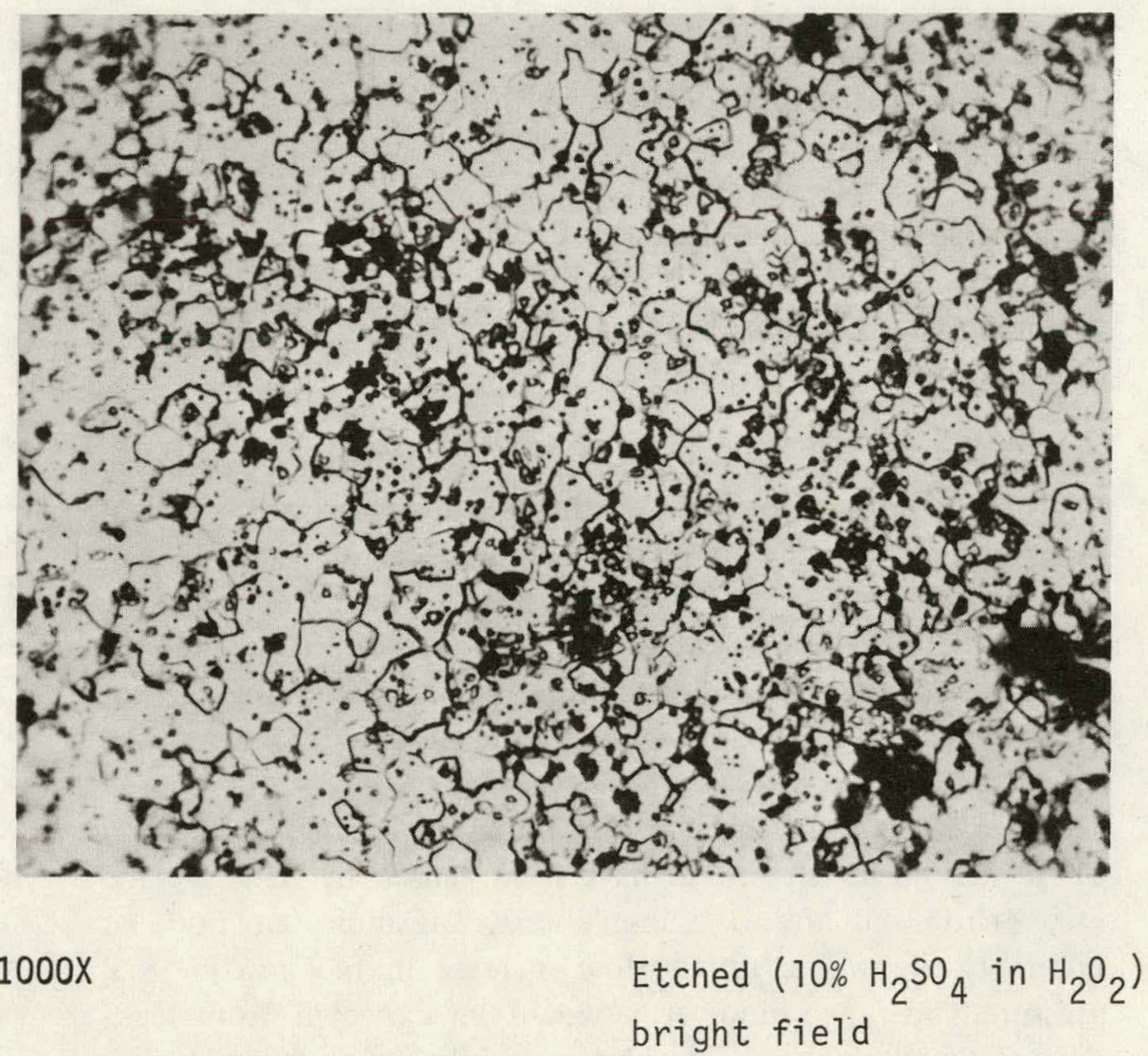

Fig. III-17 Transverse section of fuel rod showing fuel structure near outside diameter of fuel pellets 30-1/4 inches from bottom of rod. Average grain diameter measured by intercept method (uncorrected) is $3.4 \mu \mathrm{m}$ (PCM-20 Test 8-1 RS). 


\subsection{Summary of PIE Results}

The conclusions that can be drawn from the postirradiation examination are:

(1) Extensive cladding oxidation occurred over the 11-inch long region of the fuel rod from 20 to 31 inches above the bottom of the fuel rod.

(2) Cladding collapse occurred over the region 20 to 31 inches from the bottom of the fuel rod. This collapse occurred prior to the oxidation.

(3) Cladding surface temperatures up to $700^{\circ} \mathrm{F}$ higher than indicated by the cladding surface thermocouples occurred near the thermocouple locations. This was determined hy metallngraphic examination of the cladding.

(4) The thermocouple sheaths and sheath extensions act as cooling fins removing heat from the cladding and cause large temperature gradients near the point of attachment.

(5) Oxygen-stabilized alpha zirconium occurred both at the outside and inside diameters of the cladding. The source of the oxygen for the inside surface was most likely the $\mathrm{UO}_{2}$.

(6) The two breaks through the fuel rod were by brittle fracture. These are both believed to have been caused by handling the very brittle rod after the in-pile test. The indicated mode of failure in-pile was a ratler slow increase in fuel rod internal pressure. This type of indication would be expected from leaks through small cracks. A complete rod failure would result in an abrupt change in the fuel rod internal pressure.

(7) The $\mathrm{UO}_{2}$ fuel underwent considerable restructuring. This consisted of grain growth that increased towards the higher temperatures at the center of the fuel rod. 
TABLE III-I

PCM-20 TEST 8-1 RS

FUEL ROD CENTERLINE TEMPERATURE CALCULATED :FROM

GRAIN SIZE MEASUREMENTS.

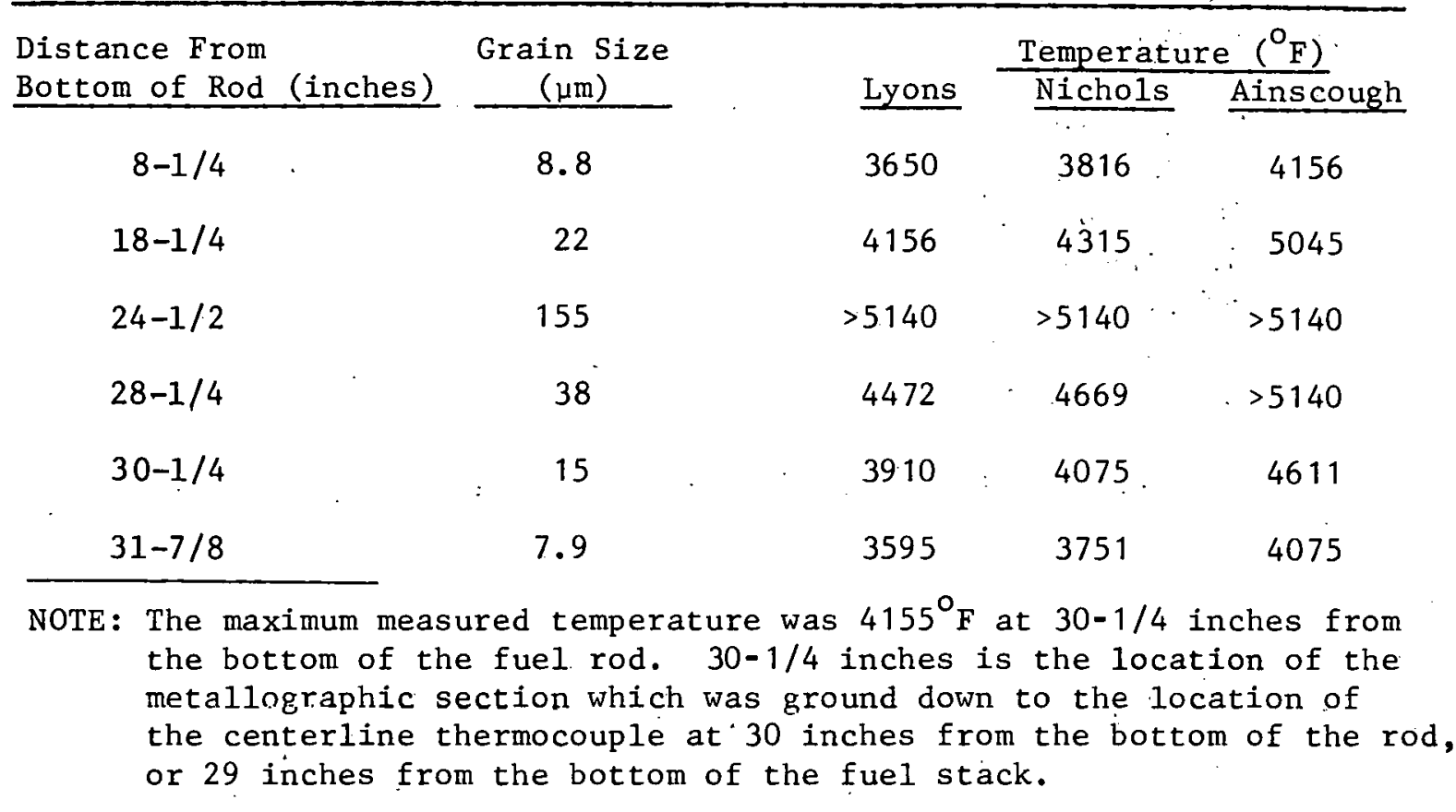

2. PCM-20 CHF SCOPING TEST, PRELIMINARY

TEST RESULTS

J. R. Larson, Z. R. Martinson, and G..W. Gibson

\subsection{Introduction}

The objectives of the PCM-20 CHF Scoping Test are delineated in Reference III-11. The CHF scoping test is the third test to be conducted in the PCM-20 test series in the PBF reactor. This scoping test was conducted to determine (a) the power level at which $\mathrm{CHF}$ occurs with a single unirradiated PWR-type fuel rod and (b) the reproducibility of the CHF phenomena. The response of the fuel and cladding following the occurrence of CHF was determined in a previous test of the PCM-20 series. A power calibration by means of an energy balance preceded the test. Selected, preliminary results of this test are presented below. Previous PCM-20 test results are presented in References III-2 and III-12. 


\subsection{Test Configuration and Instrumentation}

The test configuration consisted of a PWR-type fuel rod with a 3-foot active length of $\mathrm{UO}_{2}$ enclosed in a fuel rod flow shroud. Some basic dimensions and details of the fuel rod are as follows:

Cladding material

Cladding diameter

Cladding thickness

Diametral gap

Pellet density

$235_{U}$ enrichment

Shroud inside diameter zircaloy-4

0.422 inch

0.024 inch

0.007 inch

$9.3 \%$ theoretical

$25 \%$

0.756 inch

Other details of the rod and illustrations of a similar configuration may be found in Reference III-2.

Instrumentation of the test consisted of the following:

(1) A pressure transducer to determine the coolant pressure downstream of the test rod, and a transducer sized to measure the maximum of any pulse that might be generated from potential $\mathrm{UO}_{2}$-coolant mixing.

(2) A pressure transducer to determine the fuel rod internal pressure. This device, located external to the rod, was connected by small tubing to the upper plenum.

(3) A turbine flow meter to determinc coolant velocity entering the test section. A second turbine was located downstream of the test section to provide a backup if the inlet device should fail during testing.

(4) One chromel-alumel (Type K) thormocouple located upstiean of the inlet trubine to determine the inlet coolant temperature.

(5) A platinun resistance temperatürc device (R'I') tu delermine the temperature rise of the coolant across the test section. This was used primarily for determining the engery balance during the power calibration. Two chromel-alumel (Type K) thermocouple pairs connected to provide the tempcraturc rise were alsu installed as backups to the RTD.

(6) Thermocouples of tungsten, tungsten rhenium wire (one wire of $\mathrm{W}$ and $5 \% \mathrm{Re}$; second wire of $\mathrm{W}$ and $26 \% \mathrm{Re}$ ) in a zircaloy sheath. 
were mounted on the rod cladding with junctions at $19,21,25$ and $26-1 / 4$ inches above the bottom of the active rod length. The thermocouple leads were located $90^{\circ}$ apart on the circumference. Dummy leads of identical size were attached to the cladding upstream of the thermocouples. 'The design of the thermocouples is described in Reference III-2. For this test the thermocouple sheath was welded to the cladding completely around the tip of the thermocouple. The thermocouples were used to determine the time and location of $\mathrm{CHF}$.

(7) A 0.062-inch diameter thermocouple with the same core material as the surface-mounted thermocouples (but with a tantalum sheath) located in a 0.070 -inch diameter hole in the pellet centerline to measure fuel temperature at $26-3 / 4$ inches above the bottom of the active rod length.

(8) A strain gage located on the cladding in the rod plenum region to determine the occurrence of cladding failure.

(9) A linear variable differential transformer (LVDT) to determine the cladding axial growth and indicate the occurrence of CHF.

(10) A $0.51 \%$ cobalt-aluminum flux wire located on the outside of the fuel rod flow shroud to determine the axial distribution of the neutron flux along the fuel rod.

\subsection{Power Calibration}

For the power calibration phase of the test, the axial peak powers were varied from. 8 to $20 \mathrm{~kW} / \mathrm{ft}^{[\mathrm{a}]}$. The nominal flow conditions were a mass flux of $1.7 \times 10^{6} \mathrm{lb} / \mathrm{hr}-\mathrm{ft}^{2}$, an inlet temperature of $592^{\circ} \mathrm{F}$, and pressure of 2,200 psia. These conditions were selected to ensure a subcooled condition at the test section outlet. A single calibration was made at a peak power of $1.3 \mathrm{~kW} / \mathrm{ft}$ to obtain a data point at low power, for which the same nominal flow conditions as the CHF scoping portion of the test were used, i.e.. mass flux of $1.0 \mathrm{x}$ $10^{6} \mathrm{lb} / \mathrm{hr}-\mathrm{ft}^{2}$, inlet temperature of $620^{\circ} \mathrm{F}$, and pressure of 2,200 psia. The calibration related the test rod power to the PBF core power as indicated by current from an ion chamber located near the core.

Calculations were made with FRAP-T1 ${ }^{[b]}$ to predict the fuel centerline temperature, rnd internal pressure, and the rod axial expansion. These calculations are compared with the preliminary data in Table III-II.

[a]. The measured axial power profile has a 1.35 peak-to-average value.

[b] FRAP-T1 MODO02 EXPO4A, MATPRO MOD02 subcode. 


\section{TABLE III-II}

SUMMARY OF POWER OALIBRITION RESULTS FOR CHF SCOPING TEST

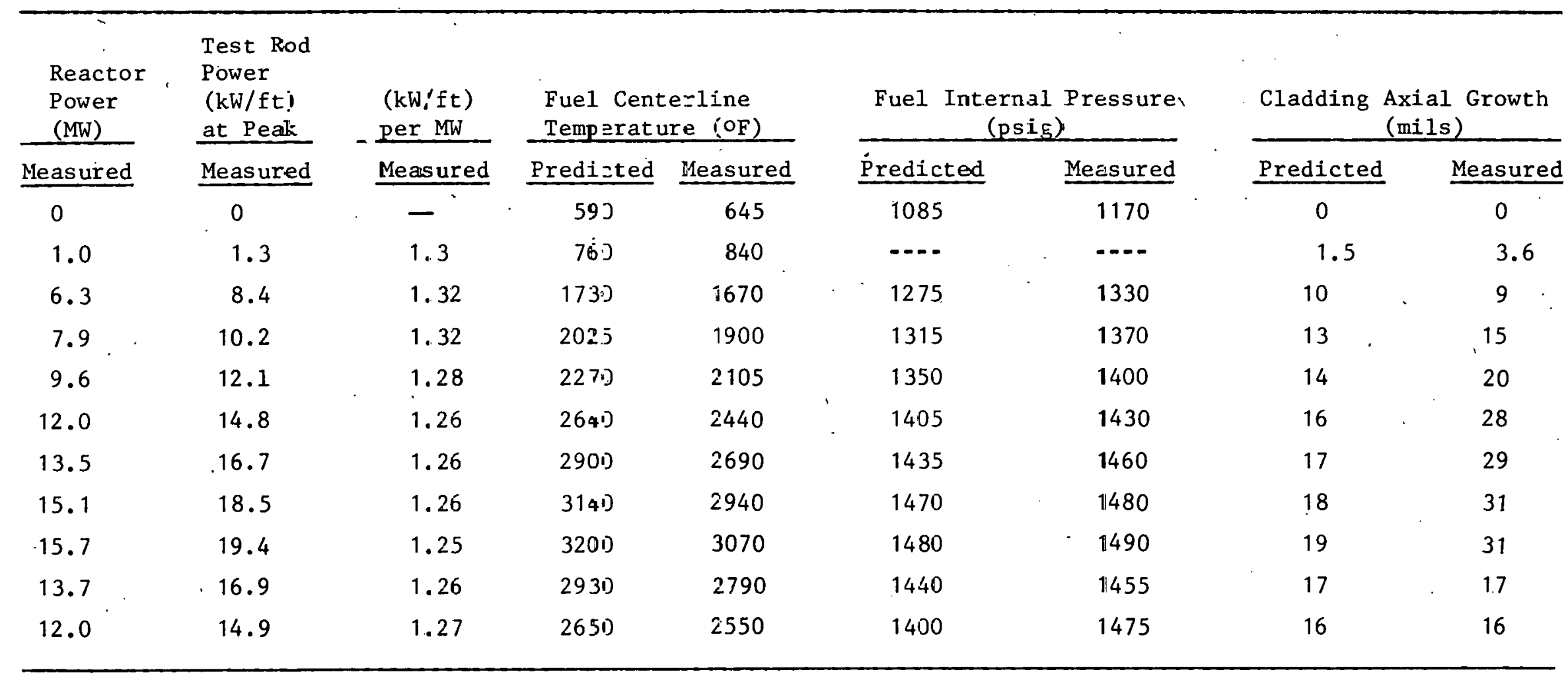


- The conditions for each calibration point were maintained for about 10 minutes. The : power at $17 \mathrm{~kW} / \mathrm{ft}$ was held constant for about one hour to determine the effects of power operation on data reproducibility.

Previous reactor physics calculations had indicated that the rod power was not strongly affected by the coolant temperature or the magnitude of voiding in the coolant channel. Thus, the calibration data determined with a subcooled liquid would be expected to apply to the CHF tests where voiding was present in the channel.

\subsection{CHF Scoping Tests}

The scoping tests were conducted by increasing fuel rod power in steps of $1 \mathrm{~kW} / \mathrm{ft}$ over a one-minute period from a nominal $15 \mathrm{~kW} / \mathrm{ft}$ in each of seven power cycles. CHF was noted by a change in temperature as indicated by one of the thermocouples located on the cladding, or by fuel rod axial growth as indicated by the LVDT. The power was held constant for about 10 minutes at each step before proceeding to the next step. The nominal inlet coolant conditions were a temperature of $622^{\circ} \mathrm{F}$, a pressure of $2,100 \mathrm{psia}$, and a mass flux of $1.0 \times 10^{6} \mathrm{lb} / \mathrm{hr}-\mathrm{ft}^{2}(6.5 \mathrm{gpm})$. Results for the seven CHF power cycles are presented in Table III-III.

During the first power cycle, CHF was exceeded while the rod power was being increased to the initial step of $15 \mathrm{~kW} / \mathrm{ft}$ at a rate of about $8.6 \mathrm{~kW} / \mathrm{ft} / \mathrm{min}$. The results for the other power cycles were more consistent and were closer to the predicted values shown in Table III-IV. For Power Cycle 1 the coolant inlet temperature was slightly higher and mass flux was lower than values used for prediction.

CHF was initially indicated by the thermocouple located at 26-1/4 inches for all power cycles except Power Cycle 4. The thermocouple at 19 inches indicated CHF simultaneously with the one at 26-1/4 inches during Power Cycle 7.

Power Cycles 1 and 2 were terminated by insertion of the reactor control rods which rapidly reduced the rod power to a value near $10 \mathrm{~kW} / \mathrm{ft}$. Other power cycles were terminated by a scram. The onset of CHF was clearly indicated by the LVDT measurement during Power Cycle 3, 4, 6, and 7. During.Yower Cycles 2 and 5, very little axial growth was noted and it lagged the CHF indication of the surface-mounted thermocouple. The small axial growth resulted because CHF was confined to a short length of the rod. The time of CHF onset on Power Cycle 1 was not sharply defined but the LVDT measurement showed the same trend as the thermocouples.

The centerline thermocouple indicated a maximum temperature of about $3,950^{\circ} \mathrm{F}$ during Power Cycle 1. Thereafter, at higher powers it behaved erratically, indicating a broken junction or electrical short had developed. The fuel rod internal pressure measurement indicated a slight increase of 15 to 20 psi after the onset of CHF from initial values of $1,7.00$ to 1,800 psi. The initial values were somewhat higher than those calculated in Table III-III. 


\section{TABLE III-III}

SUMMARY OF EXPERIMENTAL RESULTS FOR CHF SCOPING TEST

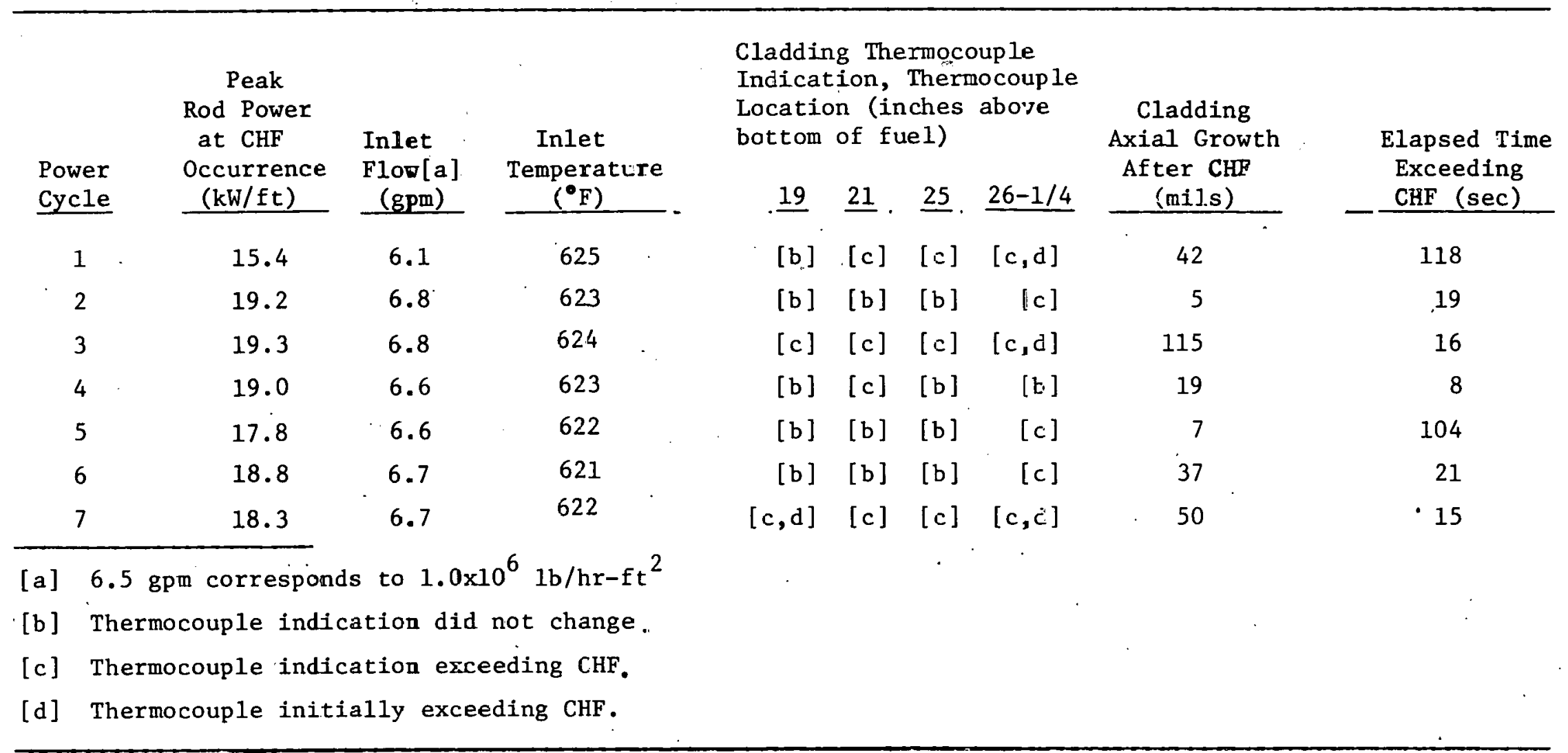


TABLE III-IV

THERMAL HYDRAULIC CONDITIONS PREDICTED AT THE ONSET OF CHF

\begin{tabular}{|c|c|c|c|c|}
\hline $\begin{array}{l}\text { CHF } \\
\text { Corre- } \\
\text { lation }[a]\end{array}$ & $\begin{array}{l}\text { Peak } \\
\text { Rod Power to } \\
\text { Reach CHF } \\
(\mathrm{kW} / \mathrm{ft}) \\
\end{array}$ & $\begin{array}{l}\text { Coolant Mass } \\
\text { Flux } \\
\left(1 \mathrm{~b} / \mathrm{hr}-\mathrm{ft}^{2}\right) \\
\end{array}$ & $\begin{array}{l}\text { Location of CHF } \\
\text { (inches above } \\
\text { bottom of fuel) }\end{array}$ & $\begin{array}{r}\text { Fue1 Rod } \\
\text { Pressure } \\
\text { (psia) } \\
\end{array}$ \\
\hline$W-3$ & 19.8 & $1 \times 10^{6}$ & $26-3 / 4$ & 1515 \\
\hline$B \& W-2$ & 23.9 & $1 \times 10^{6}$ & $26-3 / 4$ & 1580 \\
\hline LOFT & 20.6 & $1 \times 10^{6}$ & 25 & 1530 \\
\hline $\begin{array}{l}\text { [a] } \text { The } \\
\text { corr } \\
\text { resp } \\
\text { the }\end{array}$ & $\begin{array}{l}\text { Babcock and } W \\
\text { elations give } \\
\text { ectively, wer } \\
\text { fuel rod powe }\end{array}$ & $\begin{array}{l}\operatorname{cox}(B \& W-2) \\
\text { in Referenc } \\
\text { used in the } \\
\text { to attain C }\end{array}$ & $\begin{array}{l}\text { Westinghouse } \\
\text { III- } 13 \text { and III- } \\
\mathrm{AP}-\mathrm{T} 1 \text { code to }\end{array}$ & \\
\hline
\end{tabular}

\subsection{Posttest Inspection}

Following the test completion the rod was removed from the flow tube and visual examinations were conducted. Figure III-18 is a view of the rod after the test. Compared to the fuel rod used in the PCM-20 Test 8-1 RS, this fuel rod was in excellent condition.

Some bowing of the fuel rod can be detected. A small amount of cladding collapse between the fuel pellets appears to be present. In a few places the zirconium oxide coating had spalled from the rod. The relationship between the fuel rod length and the thermocouple sheaths had changed. This was indicated by bowing at the thermocouple sheaths between the tiedown straps.

\subsection{Conclusions}

Final results and conclusions will be presented in subsequent quarterly and topical reports. 

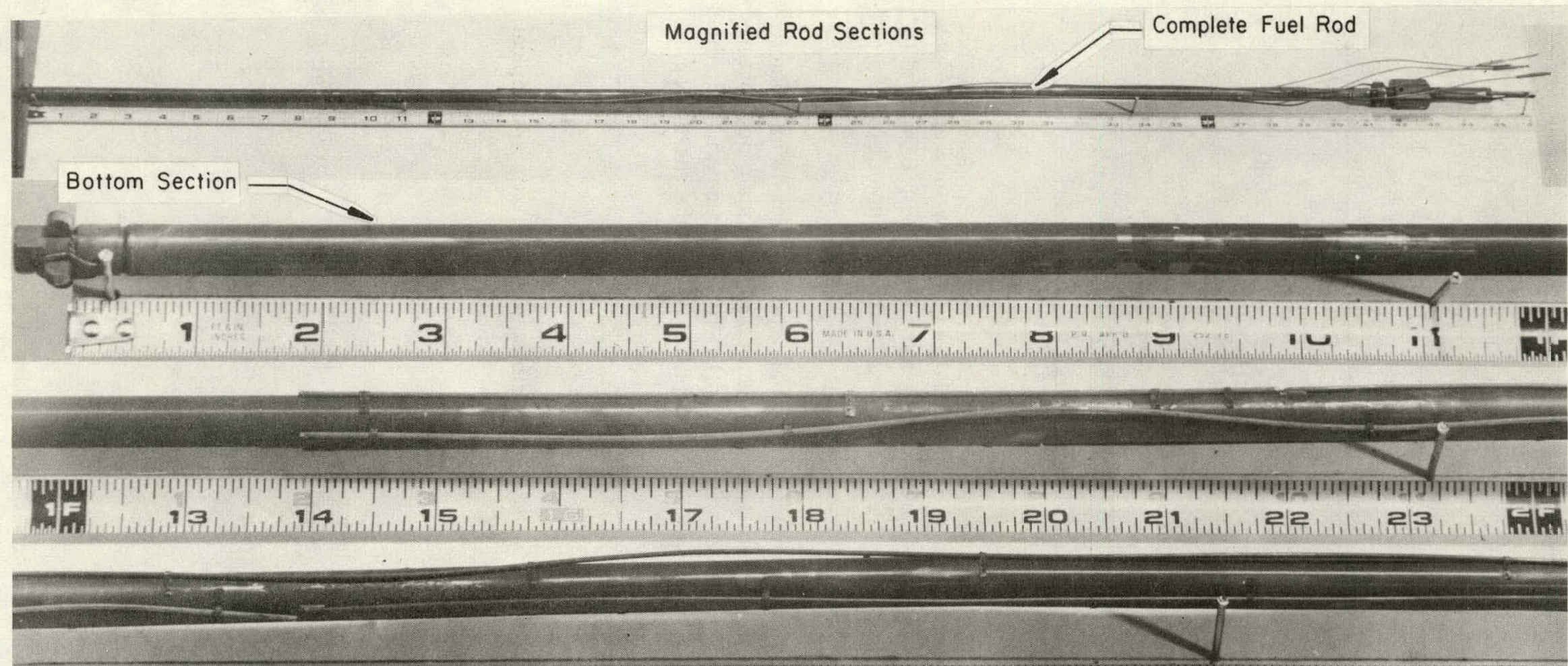

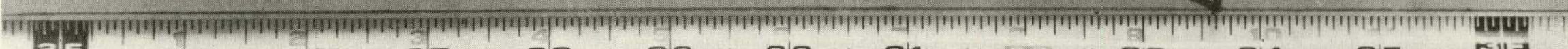

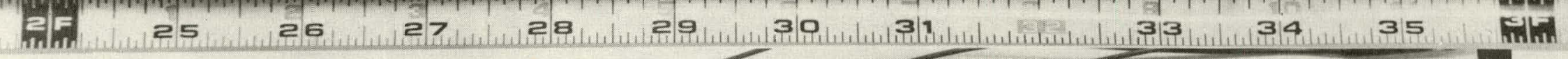

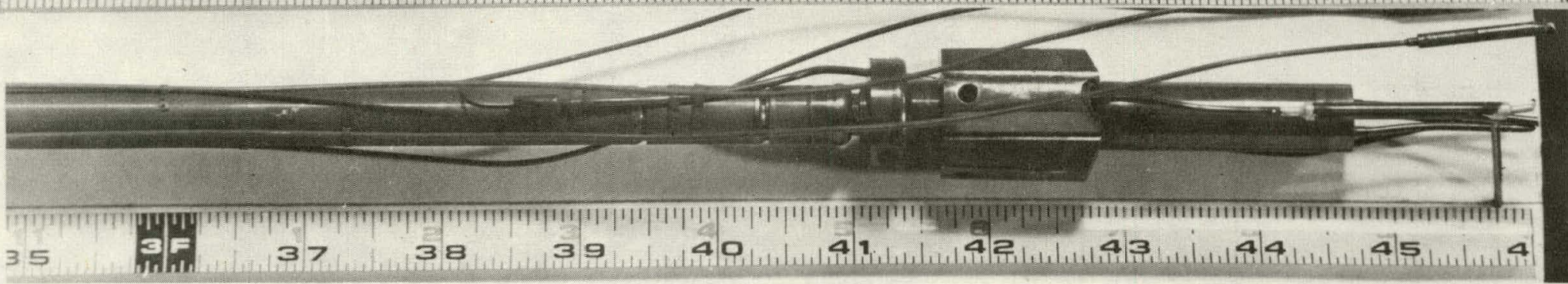

\section{A. Rod S/N UT-A0005 $90^{\circ}$ Orientation}

Fig. III-18 Fuel rod used in PCM-20 CHF Scoping Test (Sheet 1 of 2). 

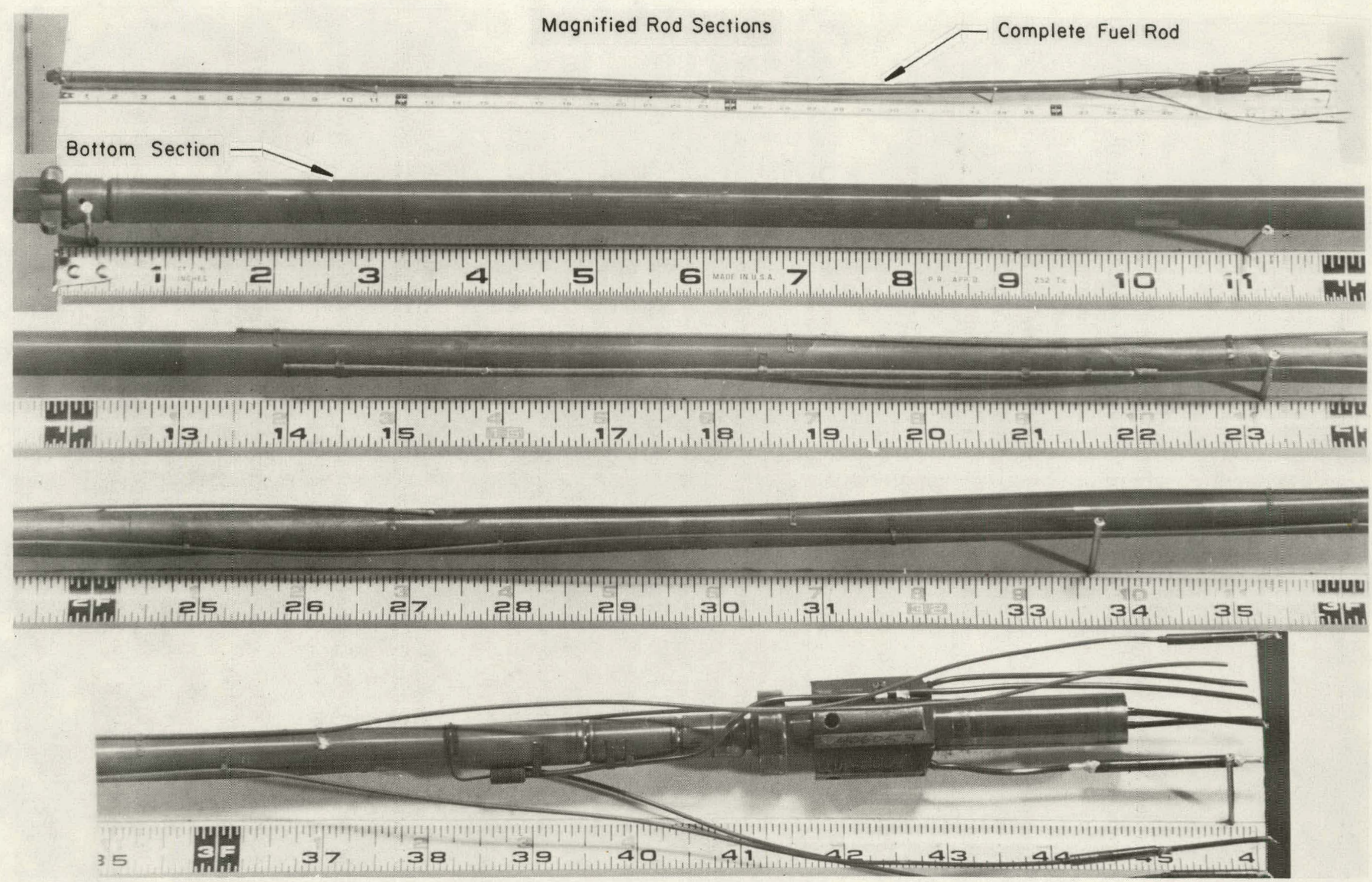

\section{B. Rod S/N UT-AOOO5 $0^{\circ}$ Orientation}

Fig. III-18 Fuel rod used in PCM CHF Scoping Test (Sheet 2 of 2). 


\section{REFERENCES}

III-1. Aerojet Nuclear Company, Quarterly Technical Report on Water Reactor Safety Programs Sponsored by the Nuclear Regulatory Commission's Division of Reactor Safety Research, January - March 1975, ANCR-1254 (September 1975).

III-2. Aerojet Nuclear Company, Quarterly Technical Report on Water Reactor Safety Programs Sponsored by the Nuclear Regulatory Commission's Division of Reactor Safety Research, April - June 1975, ANCR-1262 (November 1975).

III-3. L. N. Grossman and D. M. Rooney, Interfacial Reaction Between $\mathrm{UO}_{2}$ and Zircaloy-2, GEAP-3769 (April 1965).

III-4. D. O. Hobson, Ductile-Brittle Behavior of Zircaloy Fuel Cladding, CONF-730304 (March 1973).

III-5. R. H. Meservey and R. Herzel, Brittle Behavior of Zircaloy in an Emergency Core Cooling Environment, IN-1389 (September 1970).

III-6. D. O. Hobson and P. L. Rittenhouse, Embrittlement of Zircaloy-Clad Fuel Rods by Stearn During LOCA Transients, ORNL-4758 (January 1972).

III-7. H. M. Chung, A. M. Gards, T. F. Kassner, "Uniaxial Tensile Properties of Zircaloy-Oxygen Alloys", Paper presented at the Third Water Reactor Safety Research Meeting, Nuclear Regulatory Commission, Washington, D.C., September 29 - October 2, 1975.

III-8. J. B. Ainscough, B. W. Oldfield, J. O. Ware, "Isothermal Grain Growth Kinetics in Sintered $\mathrm{UO}_{2}$ Pellets", J. Nucl. Mat., 49 (1973-74) pp 117-128.

III-9. M. R. Lyons, D. H. Coplin, B. Weidenbaum, Analysis of UO $\mathrm{U}_{2}$ Grain Growth Data from 'Out-of-Pile' Experiments, GEAP-4411 (1963).

III-10. F. A. Nichols, "Theory of Grain Growth in Porous Compacts", J. Appl Phys, 37 (1966) pp 4599-4602.

III-11. T. G. Odekirk, Detailed Test Plan Report for PBF Test Series PCM-20: The Behavior of Unirradiated PWR Fuel Rods Under Power-Cooling-Mismatch Conditions, ANCR-1095 (April 1974). 
III-12. R. K. McCardell et al, Thermal Fuels Behavior Program Test Results Report: Power Coolant-Mismatch Series Power Calibration Test, ANCR-1248 (July 1975).

III-13. J. S. Gellerstedt, "Correlation of Critical Heat Flux in a Bundle Cooled by Pressurized Water", Two-Phase Flow and Heat Transfer in Rod Bundles Symposium, ASME Winter Annual Meeting, November 1969,pp 63-71.

III-14. L. S. Tong, "Critical Heat Fluxes in Rod:Bundles", Two-Phase Flow and Heat Transfer in Rod Bundles Symposium, ASME Winter' Annual Meeting, November 1969.pp 31-46. 


\title{
IV. REACTOR BEHAVIOR PROGRAM
}

\author{
J. H. Ramsthaler, Program Manager
}

1. INTRODUCTION

During this reporting period, the RBP conducted an in-depth evaluation of the LOCA code development and verification work being performed in support of the Division of Reactor Safety Research of the Nuclear Regulatory Commission (NRC-RSR). Current code requirements to support NRC's Water Reactor Safety Programs and licensing activities dictate that an increased emphasis be placed on the current RELAP code and that advanced models be gradually phased into the program.

RELAP4 will form the basis for a new series of codes providing greater simulation capabilities and facilitating code changes and improvements. The long-term objective is a new, completely modular RELAP in which each system component is an independent and separate module. In this light, RELAP will no longer be restricted to one hydrodynamic or component model, but will be available to apply to appropriate component and/or hydrodynamic models as needed. The near-term objectives are to maintain an operational code to develop a basic framework for total modularization, and to introduce an improved hydrodynamic model.

RBP has accordingly advanced a modified program having the following major elements:

(1) Increased effort on debugging and problem resolution for currently released versions of RELAP

(2) Initiated restructuring of RELAP to permit stepwise introduction of advanced hydrodynamic or component models

(3) Initiated efforts to study and verify (with data comparisons) any advanced components prior to incorporation in RELAP

(4) Terminated the SPLEN code development effort

(5) Initiated conversion of SCORE/EVET-MOD-1 into a production code, development of a second MOD with an interface tracking capability, and continued general SCORE coding improvements. 


\section{LOCA ANALYSIS DEVELOPMENT}

\subsection{Expansion and Tee Calculations}

\section{J. A. Trapp}

2.1.1 Introduction. This section describes the recirculating flow calculations that have been made for an expansion and tee junction with the SPLEN1 code. The code solves the conservation equations for mass, momentum, and internal energy along with two additional transport equations for the turbulent kinetic energy $q$ and the turbulent kinetic energy decay rate $\epsilon$.

Two test problems described in development of the SPLEN1 were: (a) code calculations of the Moody chart friction factors and (b) calculations of the distributions across the channel for the axial velocity and turbulent kinetic energy in a fully developed plane channel. This section describes two further calculations that have been made with SPLEN1. Calculations were made to obtain the steady state flow solution in a plane channel expansion and tee. These problems were used to check and demonstrate the capability of the code to make realistic calculations in recirculating flows. Calculations of the form and frictional losses and the thermal conductivities (heat transfer rates) were made for the expansion junction.

The basic model in SPLEN1 is summarized in 2.1.2 below along with the appropriate boundary conditions for recirculating flows. The expansion calculation is then analyzed in 2.1.3. In 2.1.4 the tee calculation is described, and 2.1.5 provides conclusions.

2.1.2 Summary and Boundary Conditions. The basic equations for mass, momentum, and internal energy in two or three dimensions are solved in SPLENl using the ICE numerical scheme. The two additional differential equations for $q$ and $\epsilon$ are solved in an explicit manner. These additional equations have terms that describe some dynamical features of the turbulence and account for convection, production, diffusion, and decay of $\mathrm{q}$ and $\epsilon$.

The boundary conditions on $\mathrm{q}$ and $\epsilon$ applied in the fully turbulent wall region just outside the transition sublayer were derived for parallel flow and are recorded here as

$$
\mathrm{q}^{2}=\frac{1}{\mathrm{C}_{\mu}}\left(\stackrel{\sigma}{\rho}_{\mathrm{w}}^{\mathrm{w}}\right)^{2}
$$

and

$$
\varepsilon=\frac{1}{y}\left[\frac{C_{\varepsilon} C_{\mu} q^{3}}{C_{D}-C_{P} \cdot \frac{\left(\sigma_{w / \rho}\right) 2}{C_{\mu} q^{2}}}\right]^{1 / 2}
$$


where $\mathrm{y}$ is the normal distance from the wall, $\sigma_{\mathrm{w}}$ is the wall shear stress, $\rho$ is fluid density, and $C_{\epsilon}, C_{\mu}, C_{D}, C_{P}$ are the constant coefficients in the $q$ and $\epsilon$ equations. Using Equation IV-1 in Equation IV-2, the boundary condition on the turbulent kinetic energy decay rate $\epsilon$ can be rewritten as

$$
\varepsilon=\left(c_{\mu}^{1 / 2} q\right)^{3 / 2} / \mathrm{Ky}, \text { or } \frac{\left(C_{\mu}^{1 / 2} q\right)^{3 / 2}}{\mathrm{Ky}}
$$

where

$$
K=\left[\frac{C_{\mu}^{1 / 2}\left(C_{D}-C_{P}\right)}{C_{\varepsilon}}\right]^{1 / 2}
$$

which is the Karman constant.

Equations IV-1 and IV-3 give the asymptotic solutions of the q and $\epsilon$ equations in the constant stress wall region for parallel flows. These solutions must be generalized when recirculating flows are examined. It is known that the turbulent kinetic energy $q$ has a maximum in the near wall region and goes to zero on the wall where the fluctuating velocity vanishes. This gives

$$
\frac{\partial \dot{\mathrm{q}}}{\partial \mathrm{x}} \mid \begin{aligned}
& =0 \\
& \text { boundary }
\end{aligned}
$$

in this region and there is no resulting diffusion of $q$ toward the wall. We use this fact and calculate $\mathrm{q}$ in the wall cell from the full $\mathrm{q}$ equation with the diffusion to the wall equal to zero; i.e., Equation IV-5 is used as the boundary condition on q. This allows q in the wall cell to be determined by the dynamics of the flow (i.e., a balance of convection, production, diffusion away from the wall and decay).

To determine the correct boundary condition for $\epsilon$ the basic ideas used to relate the turbulent eddy viscosity to $\mathrm{q}$ and $\epsilon$ are considered. The basic assumption for the eddy viscosity $\mu_{t}$ is

$$
\mu_{t}=\mu V_{t} l
$$

where $V_{t}$ is a characteristic velocity for the turbulent fluctuation and $\ell$ is a length representing the average eddy size $\left[{ }^{[V-1]}\right.$ : It has been found for parallel flows that $V_{t}$ can be taken equal to the friction velocity $\sqrt{{ }^{w} \mathrm{w} / \rho}$ and the eddy size can be taken as Ky (in the near wall region). As can be seen from Equation IV-1, in parallel flows the turbulence model gives

$$
\sqrt{\sigma_{w / \rho}}=\left(C_{\mu}^{1 / 2} q\right)^{1 / 2}
$$

In general recirculating flows, $\left(\mathrm{C}^{1 / 2} \mathrm{q}\right)^{1 / 2}$ is a better characteristic velocity for the turbulent fluctuations than $\sqrt{\mathrm{w} / \rho}$. In fact, $\mathrm{q}^{1 / 2}$ is the root mean square of the magnitude of the fluctuating velocities. $\sqrt{\mathrm{w} / \rho}$ can never represent a turbulent velocity in a 
recirculating flow region where the fluctuating velocities are large and $\sigma_{w}$ may vanish (i.e., at the reattachment point). In parallel as well as recirculating flows, $\left(C_{\mu}^{1 / 2} q\right)^{1 / 2}$ is proportional to the intensity of the velocity fluctuations and should be used as the characteristic velocity in Equation IV-6. Using Equation IV-7 in Equation IV-6 gives

$$
\mu_{t}=K y\left(C_{\mu}^{1 / 2} q\right)^{1 / 2}
$$

as the appropriate eddy viscosity in the wall region for both parallel and recirculating flows. In the two-parameter model used in SPLEN-1, the eddy viscosity is given throughout the flow field by

$$
\mu_{t}=C_{\mu} \frac{q^{2}}{\varepsilon}
$$

Since both Equations IV-7 and IV-8 hold in the wall region, the expressions can be equated for $\mu_{\mathrm{t}}$ to obtain. 1

$$
\varepsilon=\frac{\left(C_{\mu}^{1 / 2} q\right)^{3 / 2}}{K y}
$$

as the value assumed by $\epsilon$ in the near wall region.

It should be noted that the derivation of the boundary conditions for parallel flows showed that in the wall region $q$ was given by Equation IV-1 and was independent of $y$. Therefore, even for parallel flow problems Equation IV-5 is a boundary condition consistent with the basic equations for $q$ and $\epsilon$. Note also that Equation IV-10 for recirculating flows is exactly the same as Equation IV-3 used for parallel flows. Therefore, Equations IV-10 and IV -5 are the boundary conditions that should be used if both parallel and recirculating flows are present.

2.1.3 Expansion Junction. In this section the results from a SPLEN1 calculation of a two-dimensional expansion junction are presented and discussed. The dimensions of the computational region can be seen in Figure IV-1. The inlet Reynolds number based upon the inlet channel width, $\mathrm{D}_{\mathrm{in}}$, and the maximum velocity, $\mathrm{U}_{\max }$, was $2.0 \times 10^{5}$. The fluid was slightly subcooled water at about $14.7 \mathrm{psi}$. The channel expansion has an area ratio $\sigma$ equal to 0.5 . A total of 310 computational cells were used; with 31 cells in the axial direction and 10 cells across half the larger channel. The problem was run isothermally. The inlet profiles for the axial velocity, $q$, and $\epsilon$, were that obtained by SPLEN1 in a fully developed straight channel. The results of the calculation when steady state was reached are seen in Figures. IV-1 through IV-4.

Figure IV-1 shows the axial velocity profile at several representative cross sections. The general features of the flow field are easily discernible; high velocities at the throat, a recirculation region downstream from the expansion ending at the reattachment point 


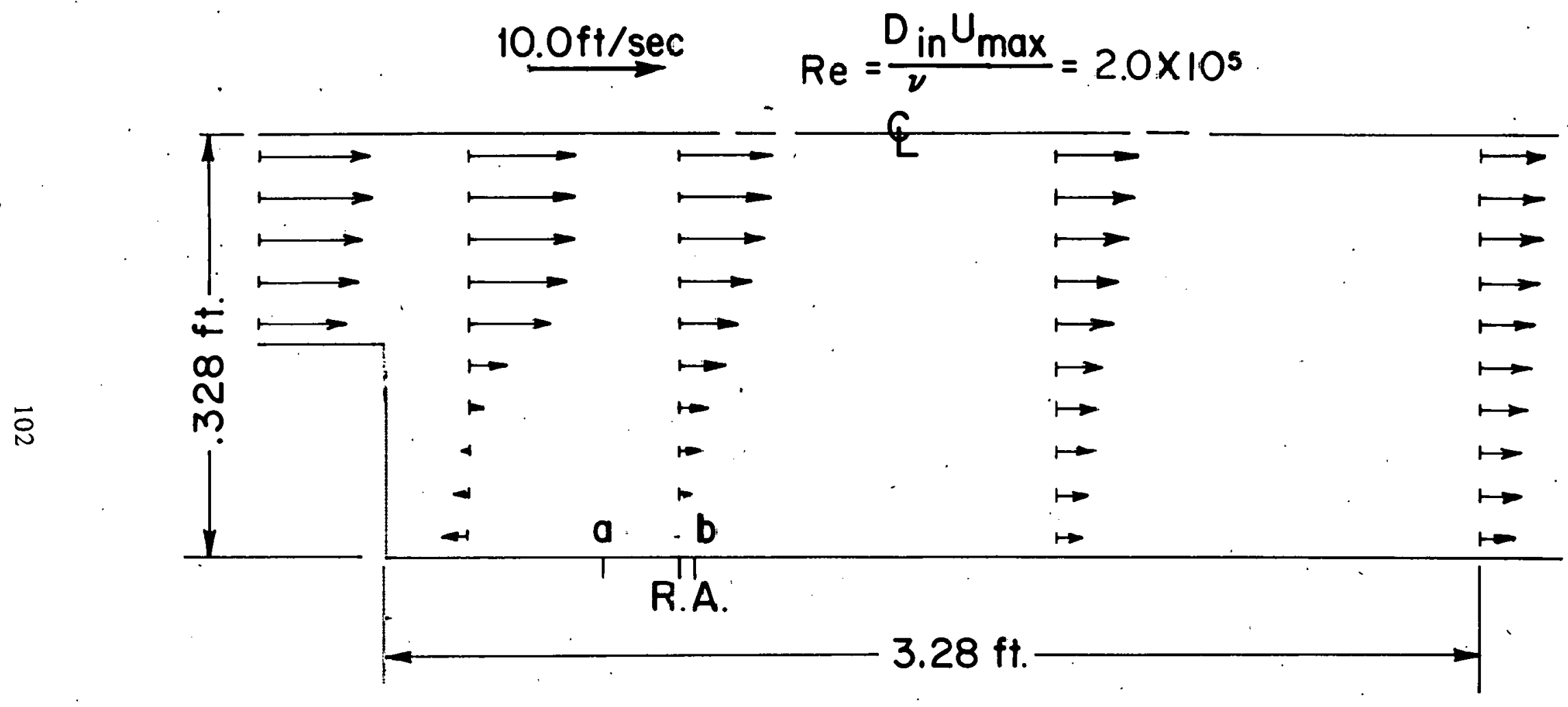

Fig. IV-1 Axial velocity profile of two-dimensional expansion junction. 

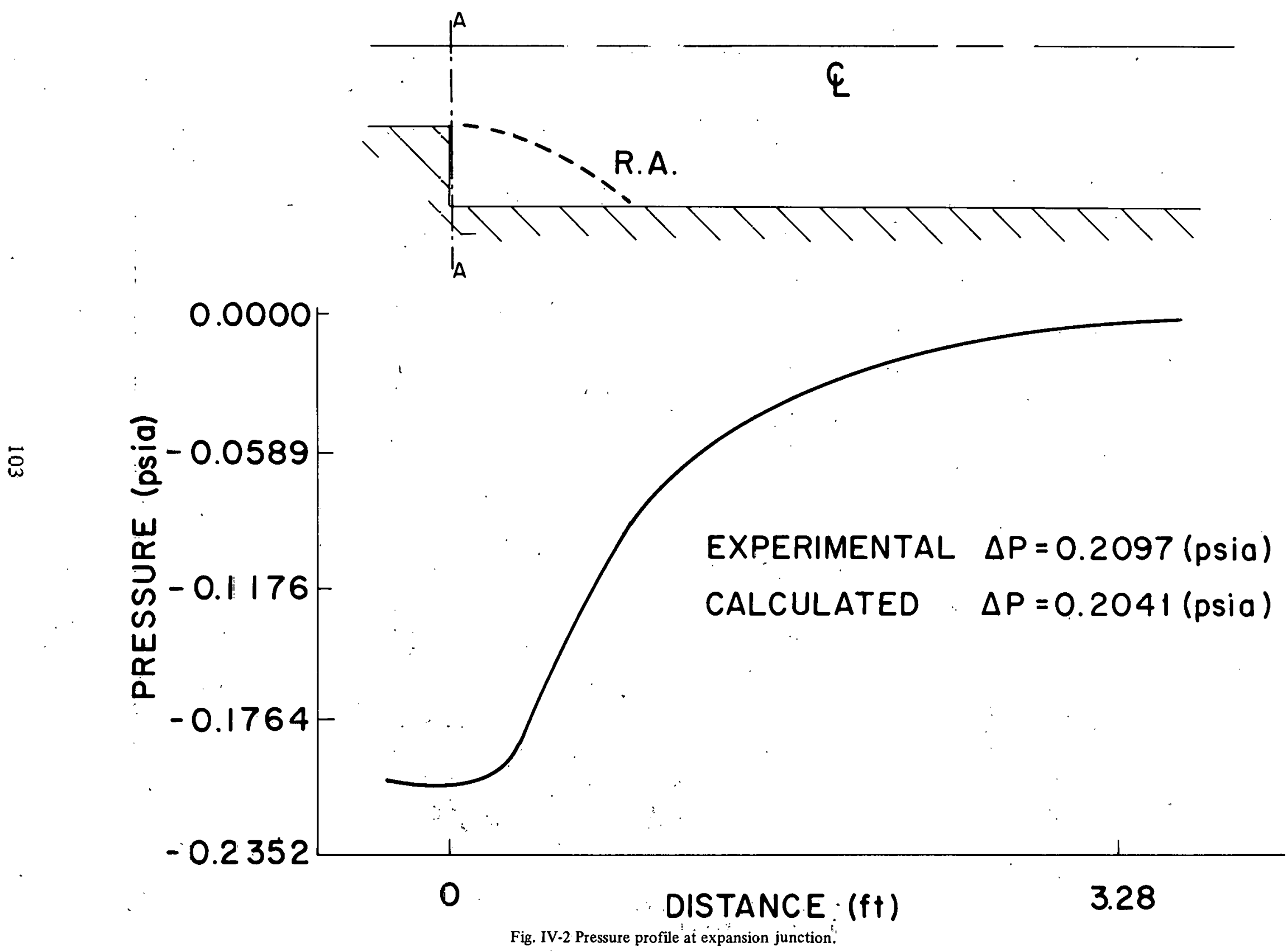


\section{q PROFILE}

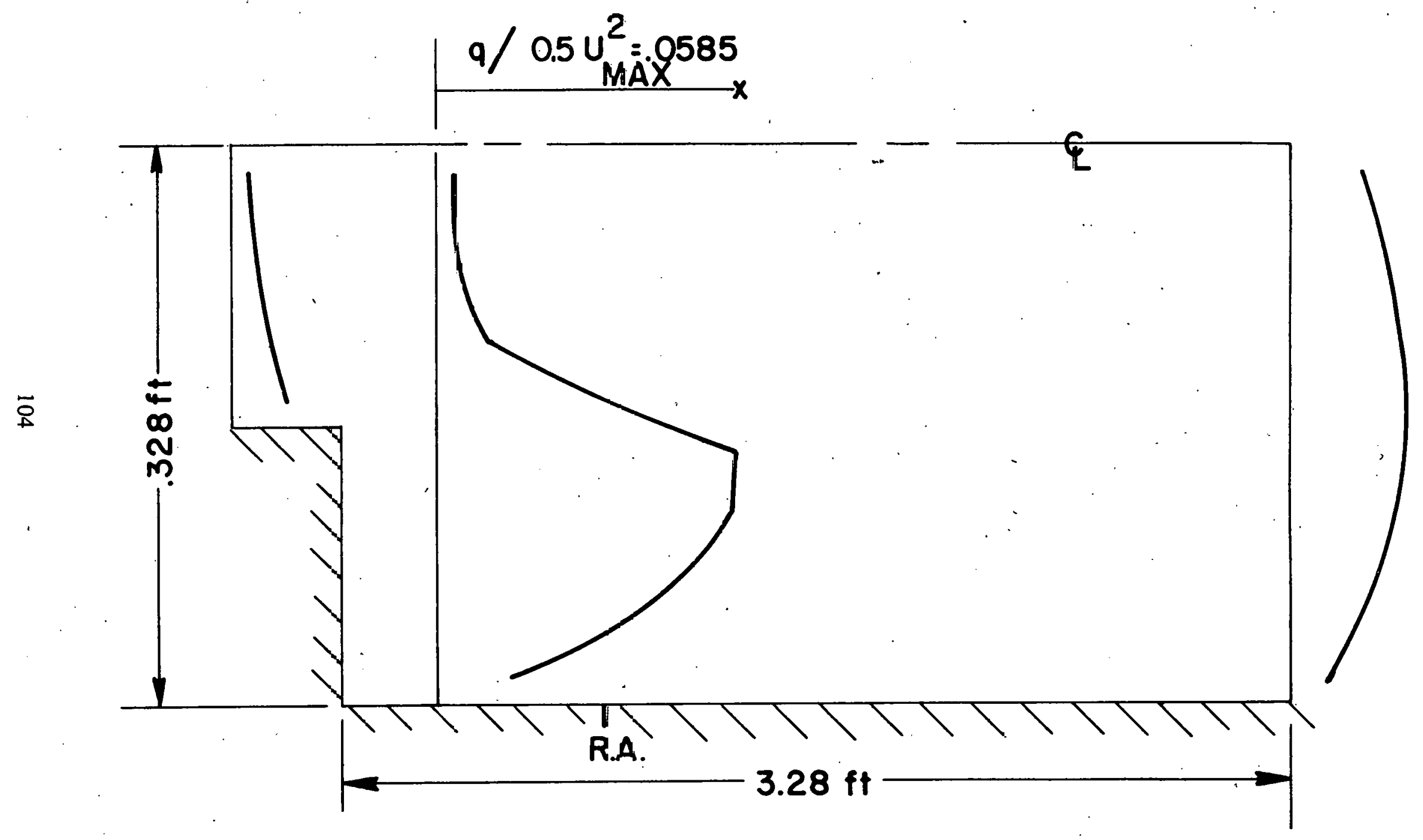

Fig. IV-3 Turbulent kinetic energy q profile. 


\section{WALL SHEAR STRESS (WALL CONDUCTIVITY)}

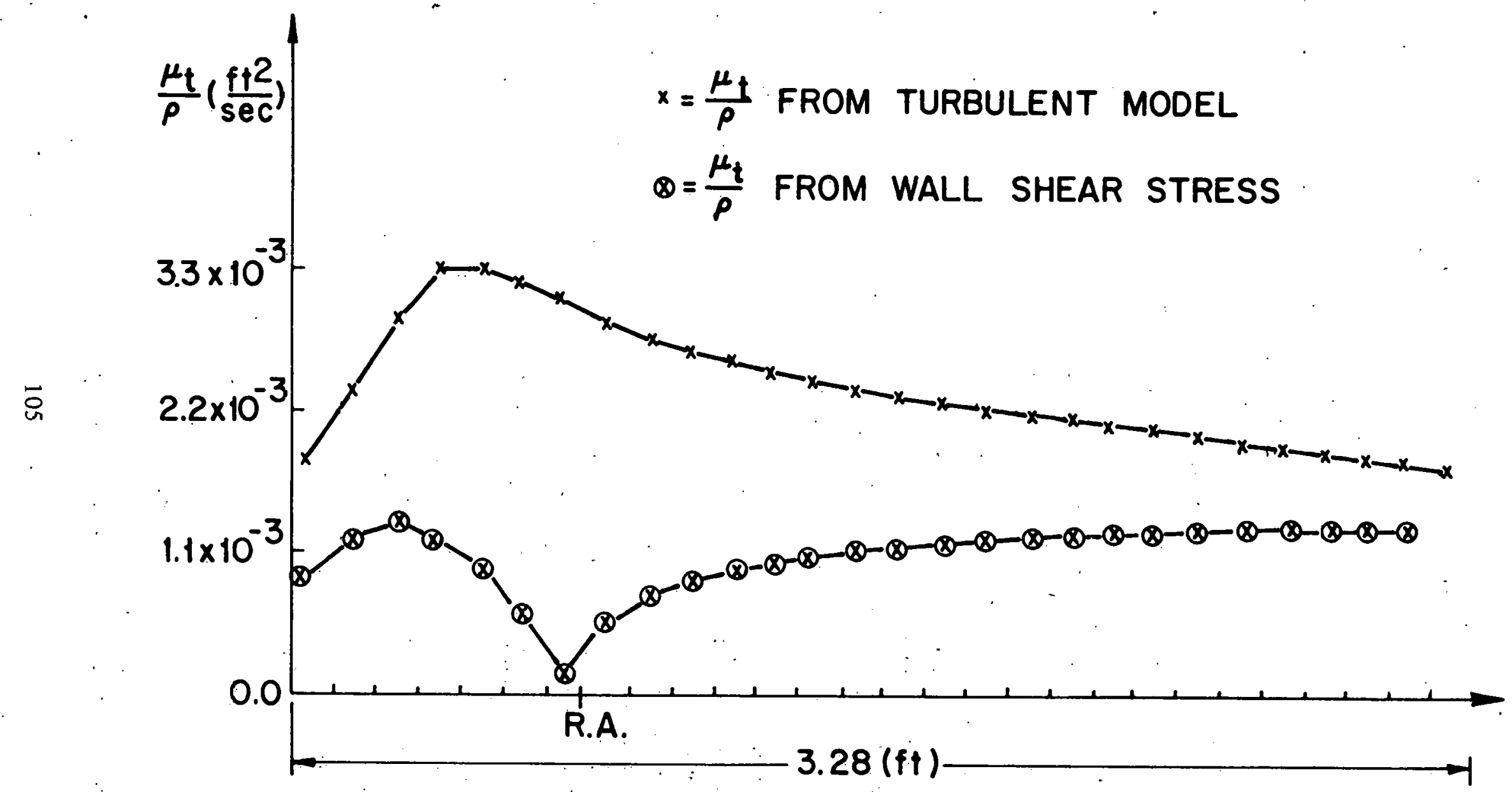

Fig. IV4 Turbulent viscosity $\mu_{t}$ in near. wall region. 
(R.A.), and a redeveloping velocity field beyond the R. A. point. The points shown as a and $\mathrm{b}$ in Figure IV-1 give the limits within which the experimental data for the R. A. point falls [IV-2,IV-3,IV-4]. The calculated R. A. point lies well within the scatter from the experimental data.

Figure IV-2 shows the pressure rise along the center line as calculated by SPLEN1. The total pressure rise agrees very well with the expenmental data and rises to within $2-1 / 2 \%$ of the total pressure drop obtained from the Borda loss equation. The pressure on the vertical step of the expansion surface is assumed constant and equal to the throat pressure in the derivation of the Borda loss equation. The calculated pressure variation, $P_{\max }-P_{\min }$, along the throat section AA was less than $2 \%$ of the total pressure drop across the expansion. This simple numerical verification of a, nearly uniform pressure at the expansion section shows how the code can be used to check the assumptions made in simplificd junction modcls. It should be noted that the code calculated nearly uniform pressures across the channel at all cross sections. This is also consistent with the experimental data $[I V-2]$.

Figure IV-3 shows the turbulent kinetic energy profile across the channel at several representative locations. The increase of $\mathrm{q}$ in the recirculation region and especially on the interface between the recirculation zone and the jet core region is qualitively in agreement with the circular pipe expansion data of Reference IV-2. The large value of $q$ in this "interface". region is due to the large value of the production terms in the q equation caused by the large mean shear gradients in this region.

The maximum value of $q / 1 / 2 U_{\max }^{2}$ in the recirculation zone of Chaturvedi's [IV-2] pipe flow data $\left(\sigma=0.25, \mathrm{Re}=2.0 \times 10^{5}\right)$ was 0.0532 , whereas the maximum value in the channel flow calculation $\left(\sigma=0.50, \operatorname{Re}=2.0 \times 10^{5}\right)$ was 0.0585 . Hence even the quantitative value of the calculated maximum turbulent kinetic energy is in reasonable agreement with the data. In Reference IV-2; the measured q profiles have the same basic shape as seen in Figure IV-3. These results concerning the q profile show that the local turbulent parameters are being reasonably well modeled by the $\mathrm{q}$ and $\epsilon$ equations.

The calculation shows, as expected, the longer development lengths for the turbulent parameters. At the end of the computational region where the pressure and velocity have reached their fully developed values we can see from Figure IV-3 that $q$ is still developing. When the q profile becomes fully developed downstream it will have a profile similar to the inlet profile. The change in shape of the $q$ profile between the recirculating region and the end of the computational region is brought about by the diffusion terms in the q equation.

Figure IV -4 gives a profile of the turbulent viscosity. $\mu_{t}$ in the near wall region, i.e., in the computational cell on the wall. From Figure IV 4 it is seen that the turbulent viscosity

[a] The experimental data in these references was for circular pipe expansions with different area ratios. These data and the SPLEN1 plane channel expansion were compared when the area ratio in the pipe expansions was 0.5 . 
in the recirculation zone is about 2.5 times larger than the fully developed value downstream ${ }^{[a]}$. Recalling that the turbulent conductivity in the two-parameter model is related to $\mu_{\mathrm{t}}$ by

$$
\mathrm{k}_{t}={ }^{\mu_{t / P}}\left(.6 \leq \mathrm{P}_{\mathrm{r}} \leq .9\right)
$$

then the same remarks made concerning $\mu_{\mathrm{t}}$ can be made concerning $\mathrm{k}_{\mathrm{t}}$. Hence the turbulent conductivity in the recirculation region is about 2.5 times larger than its fully developed value. Although the expansion problem was run with constant internal energy and hence no heat flux, the heat transfer rates to the wall that would have been calculated in a general problem with wall heat flux will show the same trends as $k_{t}$ does in the constant energy problem. This increase in the heat transfer rate in the recirculation zone has been noted by a number of experimental investigations $[$ IV-5, IV-6]. In Reference IV-7 it is stated that rates of heat transfer two to four times higher than the fully developed values are found in the recirculating region. This is in the range calculated from the code results (assuming the heat transfer rates are proportional to $\mathrm{k}_{\mathrm{t}}$ ).

The calculated increase in the thermal conductivity (and attendant heat transfer rate) may be important in core blockage problems. If a reduction in flow area caused by a partial blockage (i.e., ballooning of the cladding, etc.) is followed by an expanding flow area and attendant recirculation, then we may expect increased cooling in the expanded region. This may help to keep the temperature in the vicinity of the blockage from rising to an undesirable value.

Instead of using a differential model of turbulence, an algebraic prescription could have been made. If the mixing length formula for pipe or channel flow is used, in which it is assumed that $\mu_{\mathrm{t}}$, is proportional to the normal distance from the wall and the friction velocity $\sqrt{\sigma_{\mathrm{w}} / \ell}$, then from dimensional consideration and experimental results:

$$
\mu_{t}=K_{y} \sqrt{\sigma_{w} / \rho}
$$

When the code calculation of $\sigma_{\mathrm{w}}$ is used in Equation IV-12, the turbulent viscosity shown by the circles in Figure IV-4 is obtained. This would have been the turbulent viscosity (or conductivity) calculated from an algebraic mixing length formula. From Figure IV 4 it can be seen that this gives turbulent viscosities and conductivities that are completely in error. In the region about the $\mathrm{R}$. A. point where heat transfer is high, the mixing length predicts zero conductivity.

2.1.4 Tee Junction. The tee junction shown in Figure IV-5 was run with SPLEN1. The axial velocities, $\mathrm{q}$ and $\epsilon$, used at the two inlet sections were that calculated by the code for a fully developed channel flow. The velocities are typical of those expected at the junction of the pressurizer and hot leg during the first part of a slow break analysis. The mesh spacing was uniform in the transverse direction with 15 cells of size 0.03 meter. The mesh spacing

[a] The fully developed value is reached downstream from the computational region and was calculated using the friction factor from the Moody chart. 
TEE VELOCITY PLOT

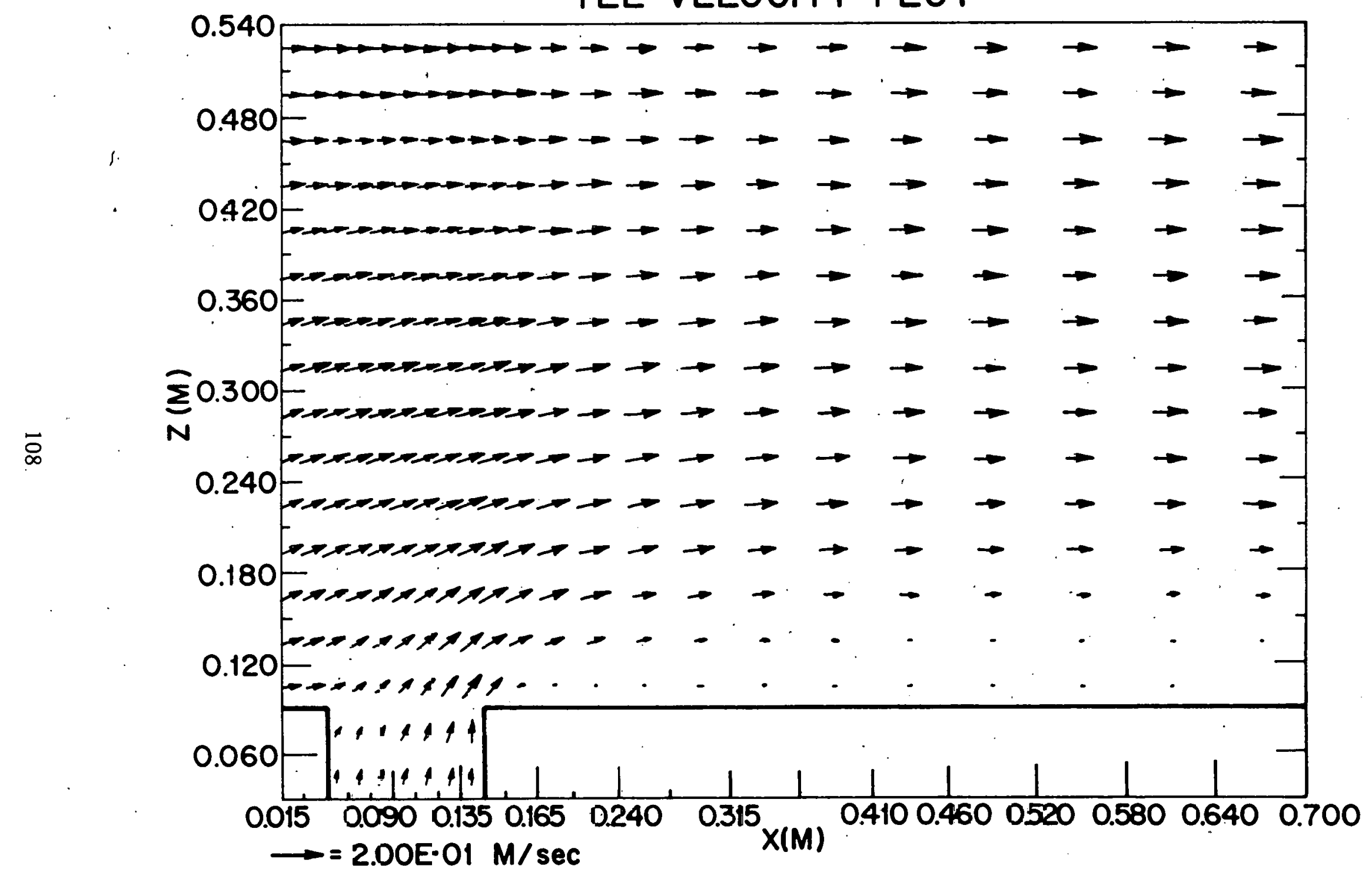

Fig. IV -5 Tee junction velocity profile. 
in the main axial flow direction was variable with the smallest cells near the junction: A total of 21 axial cells was used with the cell size varying from 0.015 to 0.060 meter.

A detailed analysis of the tee calculation was not carried out. This was due to the fact that the computational region was not as long as needed in the main axial channel and fully developed pressure drop calculations were not obtained. The basic qualitative features of the flow were as expected: a recirculation zone occurs downstream from the junction and higher velocities due to the junction flow into the main channel from the side branch.

2.1.5 Conclusions. SPLEN1 was applied to the analysis of two recirculating flow problems. The calculations were made for a plane expansion junction and a plane tee junction with the appropriate boundary conditions.

A detailed analysis of the expansion calculation and experimental data has shown SPLEN1 capable of accurately predicting: (a) the pressure drop and attendant form losses in a junction, (b) the wall shear stresses and friction losses, and (c) the correct trends in the heat transfer rates.

The expansion calculation also shows how the code can be used to verify assumptions made when deriving simplified junction models. The Borda loss equation for an expansion junction is derived by assuming a constant pressure on the expansion surface and throat. The SPLEN1 calculation shows that this is indeed the case.

The calculation has shown that a two-parameter turbulent model will give realistic calculations for the turbulent viscosities and conductivities in recirculating flow regions where the algebraic mixing length formula fails.

\section{FUEL ROD MODEL DEVELOPMENT}

\subsection{BALLOON: A Model to Calculate the Transient Ballooning of Reactor Fuel Cladding} J. A. Dearien

3.1.1 Introduction. A model (BALLOON) has been developed to describe the local deformation or "ballooning" of pressurized reactor fuel cladding during a LOCA. The model calculates mechanical deformation of the cladding and interaction of the deformed cladding with surrounding coolant. This model has been developed as part of the FRAP-T code development effort.

The purpose of this model is to define the large deformation geometry of fuel rod cladding during accident conditions. These calculated deformations are required for determining thermal-hydraulic characteristics of the reactor core during recovery from an accident. In addition to determining geometry, results from the developed model will be used to determine when and where cladding rupture occurs within the core. Magnitude and distribution of cladding rupture will be used in determining the amount of fission products released to the coolant and containment. 
3.1.2 Description of Model. This section describes the model with regard to the assumptions and equations used to formulate the model and the analytical sequence-by which the model is solved.

The deformation model is shown in Figure IV-6 as a series of membrane elements defining the ballooned shape. Location of the $\mathrm{i}^{\text {th }}$ membrane element in space is defined as the radial distance, $r_{i}$, from the fuel rod centerline. Driving force for deformation of the membrane is the differential pressure across the membrane and is decreased as a function of increasing volume. Temperature of the cladding is considered as a transient function of cladding geometry and coolant conditions with cladding material properties determined as a function of local temperature.

3.1.2.1 Assumptions. The following assumptions are used in formulating and solving the response of the swelling model:

(1) Stability of the deformed shape can be described by membrane theory (in-planc forcc only).

(2) Stresses and temperatures are uniform through the cladding thickness.

(3) Axial and circumferential stresses at a point can be defined as a function of temperature, strain, and strain rate by one relationship. (Anisotropic relations are presently under development.)

(4) The centroid of each nodal element remains on an extension of the radial vector to the original centroid.

3.1.2.2 Equilibrium Equation. The equilibrium equation for the membrane element in Figure IV-6 is

$$
\frac{\sigma}{\dot{r}_{a}}+\frac{\sigma_{\theta}}{r_{c}}=\frac{p}{t_{c}}
$$

where

$$
\begin{array}{lll}
\mathrm{p} & = & \text { differential pressure } \\
\sigma_{\mathrm{a}} & = & \text { axial stress } \\
\sigma_{\phi} & = & \text { hoop stress } \\
\mathrm{r}_{\mathrm{a}} & = & \text { axial radius of curvature } \\
\mathbf{r}_{\mathrm{c}} & = & \text { circumferential radius of curvature } \\
\mathrm{t}_{\mathrm{c}} & = & \text { cladding thickness. }
\end{array}
$$




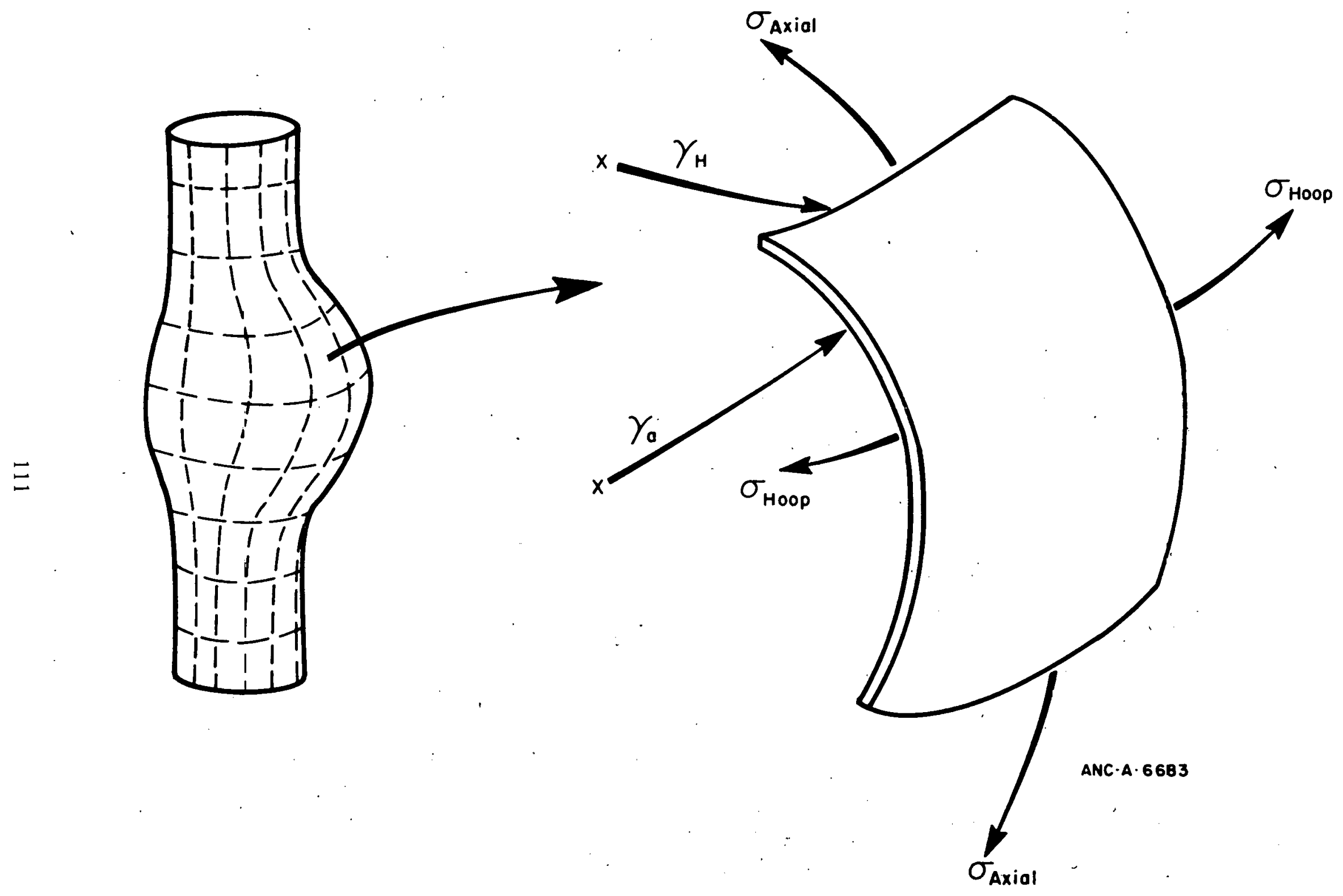

Fig. IV-6 Membrane swelling model. 
This equilibrium model is the same basic model as reported in earlier versions of the SWELL subcode. The swelling model described herein differs from previous models in the manner of solution, the model for clad yielding, and completeness of the model.

Considering the assumption that no significant deformation is obtained until both axial and radial stresses have exceeded yield stress, Equation IV-13 is expressed as

where

$$
\sigma_{y}\left(\frac{I}{r_{a}}+\frac{1}{r_{c}}\right) \cdot / \frac{p}{t_{c}}=f
$$

$$
\begin{array}{lll}
\sigma_{\mathrm{y}} & =\text { yield stress of cladding } \\
\mathrm{f} & =\text { node stability factor. }
\end{array}
$$

For a given internal pressure $P$, clad thickness $t_{c}$, clad yield stress $\sigma_{y}$, and local curvatures $r_{a}$ and $r_{c}$, the solution of Equation IV-14 determines whether an element is stable or will deform under the applied pressure. When an unstable element is detected, the cladding is deformed in such a manner as to make the system more stable. This adjustment in the geometry of the clad is described in Section 3.1.2.3.

3.1.2.3 Geometric Models. The following sections describe the assumption and formulations of relations defining the transient geometry of the swelling model.

Local Curvature. Stability of the membrane section shown in Figure IV-6 is a function of the curvatures, $r_{a}$ and $r_{c}$, in the axial and radial direstion of the swelling zone. Local curvature is calculated for each iteration in terms of the local geometry during the previous iteration. Figure IV-7 shows an axial section through a deformed section of the cladding. Node locations are noted on the deformed section relative to the node $\mathrm{i}$ for which local axial curvature (LAC) is to be calculated. The geometric relations used for calculation of LAC are noted in Figure IV-7. All calculated deformations, $d_{i}$, are measured from the original centerline of the fuel rod. Figure IV-8 shows an axial cross section through the deformed cladding and the geometric relations describing local circumferential curvature (LCC). Symmetric swelling is not assumed in the formulation. Nonsymmetric displacements are averaged in the formulation of the local curvatures.

Local Surface Area and Clad Thinning. The cladding deformation model assumes constant nodal volume during expansion. Figure IV-9 illustrates the model. Surface area of node $i$ in the deformed state is calculated as

$$
\mathrm{A}_{i}=\mathrm{d}_{i} \phi \dot{\omega}_{a_{i}}
$$




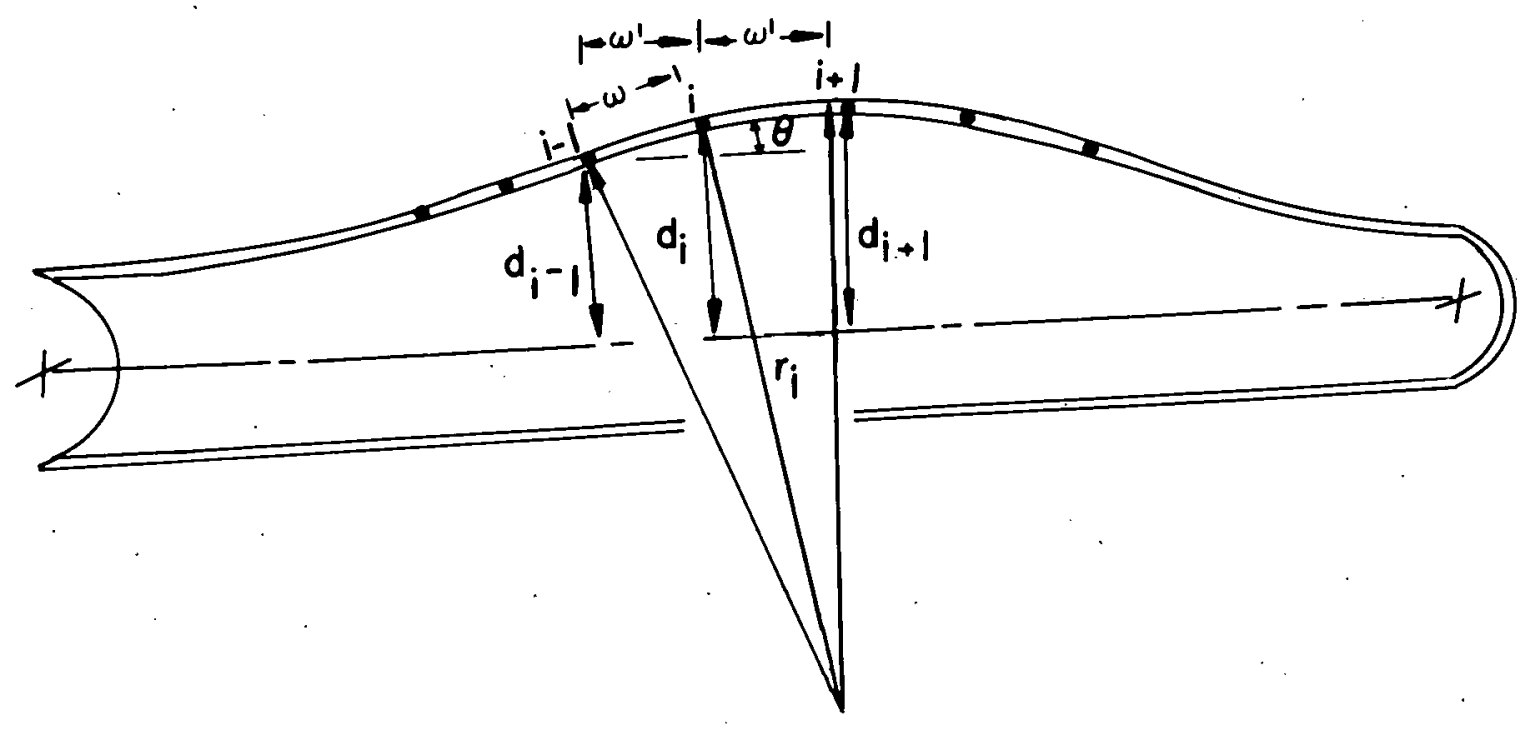

$$
\begin{gathered}
\theta=\tan ^{-1} \frac{\left(d_{i+1}-d_{i-1}\right)}{2 \omega} \\
\delta_{a i}=\left[d_{i}-\left(\frac{d_{i+1}+d_{i-1}}{2}\right)\right] \frac{1}{\cos \theta} \\
\omega=\frac{\omega^{\prime}}{\cos \theta} \\
r_{i}=\frac{\left(\delta_{a i}\right)^{2}+\left(\omega_{a i}\right)^{2}}{2 \delta_{a i}}
\end{gathered}
$$

Fig. IV-7 Calculation of local axial curvature.

where

$$
\begin{array}{lll}
\mathrm{d}_{\mathrm{i}} & =\quad \text { displacement of node } \mathrm{i} \\
\phi \quad & \quad \text { circumferential nodal spacing (radians) } \\
\omega_{\mathrm{a}_{\mathrm{i}}-} & \quad \text { axial node separation after swelling (see Figure IV-7). }
\end{array}
$$




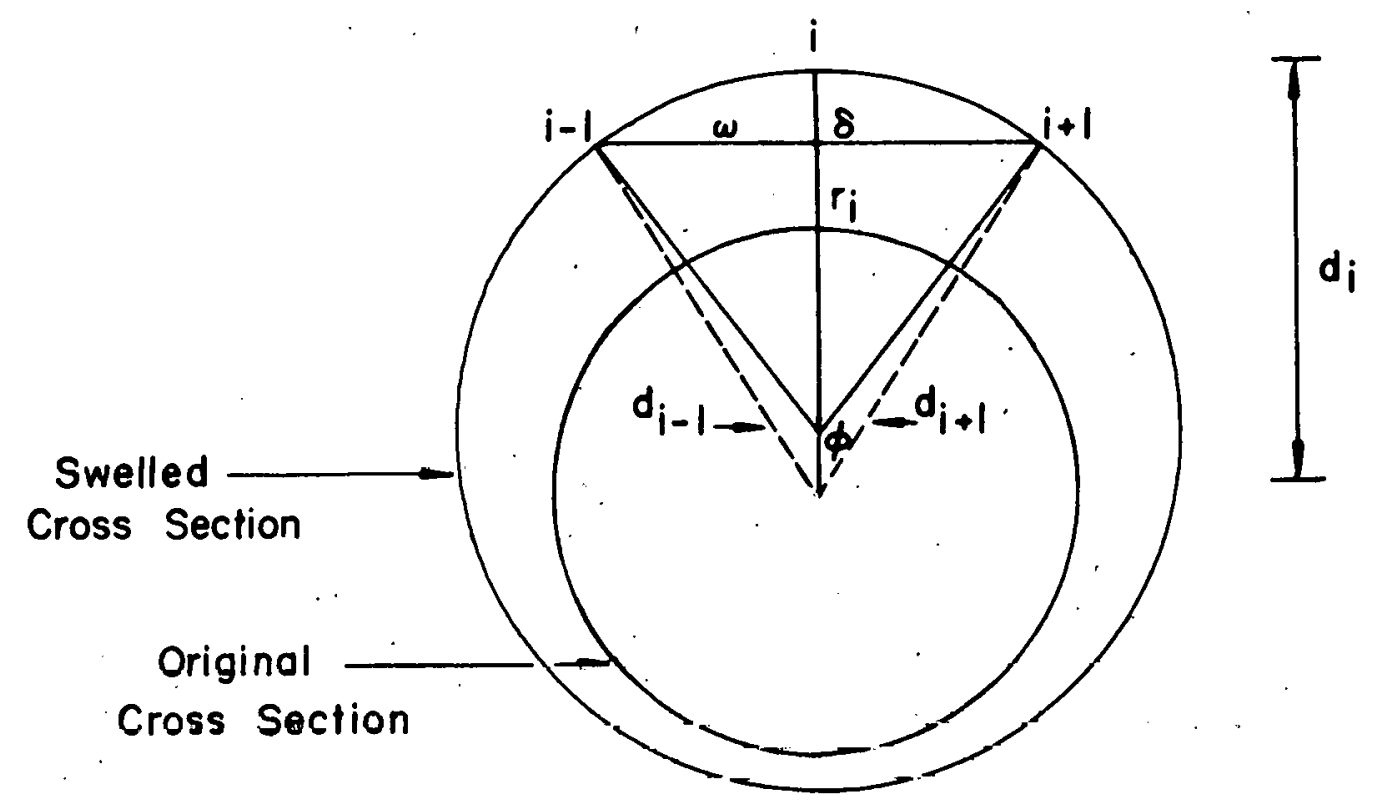

$$
\begin{aligned}
\phi & =\text { user specified angular nodal spacing } \\
\omega & =\frac{d_{i-1}+d_{i+1}}{2} \sin \phi \\
d & =d_{i}-\frac{d_{i-1}+d_{i+1}}{2} \cos \phi \\
r_{i} & =\frac{8^{2}+\omega^{2}}{28} \\
\theta & =\sin ^{-1} \omega / r_{1}
\end{aligned}
$$

Fig. IV-8 Calculation of local circumferential curvaturc.

Assuming constant nodal volume, the following relation is obtained for local cladding thickness:

$$
t_{c}=r_{o} \phi \omega^{\prime} t_{o} / A_{D_{i}}
$$

where

$$
\begin{array}{lll}
r_{0} & = & \text { original cladding radius } \\
\omega^{\prime} & = & \text { axial node spacing } \\
t_{0} & = & \text { original cladding thickness. }
\end{array}
$$




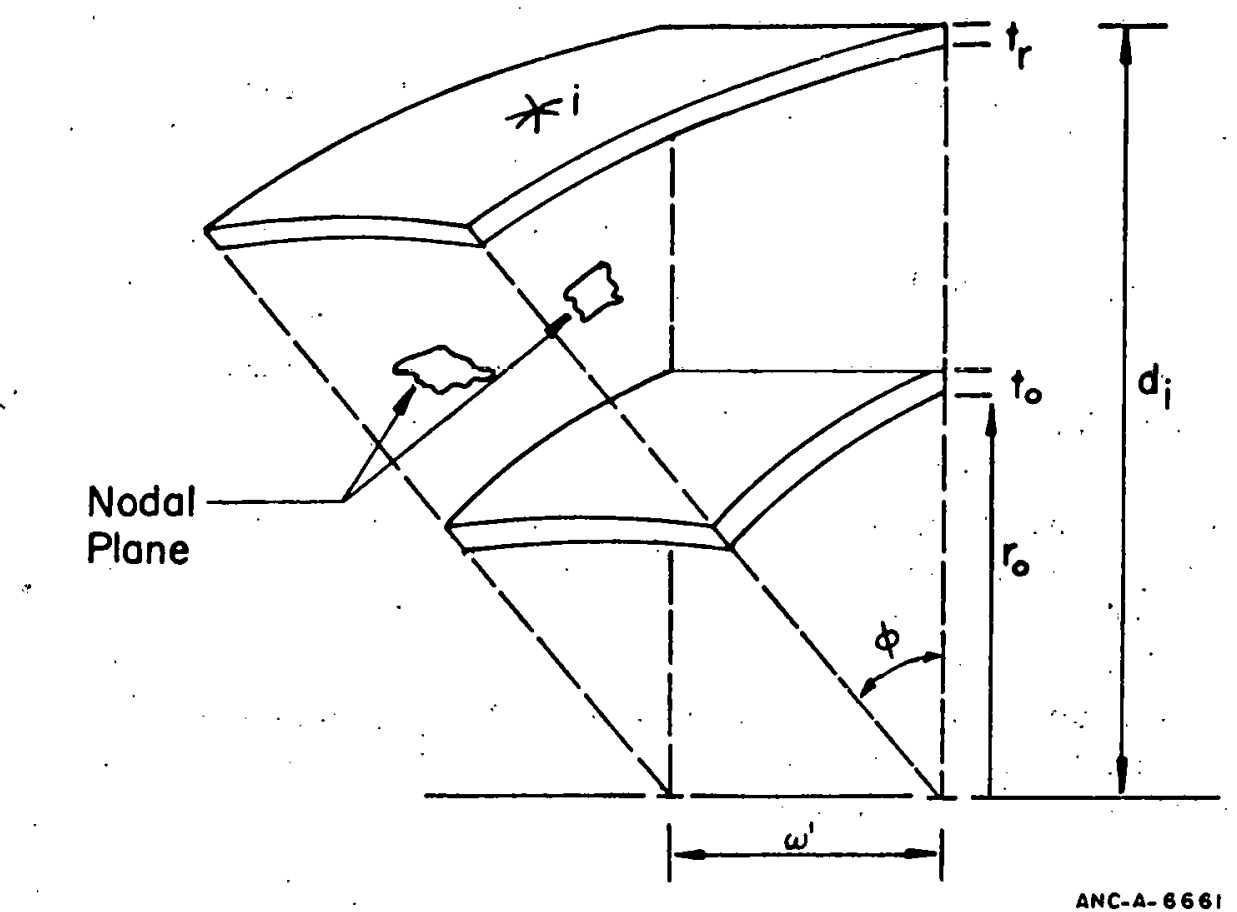

Fig. IV-9 Surface area and cladding thinning model.

The clad thinning model assumes that the cladding material contained within the axial length $\omega^{\prime}$ and angular sector $r_{0} \phi$ (Figure IV-9) remains within the confines of this three-dimensional space during expansion. Verification of this assumption that material does not cross a nodal plane will require testing in which motion of the cladding surface is measured during the test.

Internal Volume. Volume of the swelling zone is required for input to the fuel rod pressure model. Incremental nodal volumes are calculated as the node is displaced and summed to produce a new swelled volume for each time step. The relationship defining the incremental nodal volume is

$$
\Delta V_{i}=d_{i} \phi \omega^{\prime} \delta_{i}
$$

where

$$
\delta_{\mathrm{i}}=\quad \text { incremental displacement of node } \mathrm{i} \text { during the time step. }
$$

Total swelling volume for the time step is then

$$
v=v_{0}+\sum_{i=1}^{n} \Delta v_{i}
$$

where.

$$
\begin{aligned}
& \mathrm{n} \quad=\quad \text { number of nodes } \\
& \mathrm{v}_{\mathrm{O}}=\quad \text { volume from previous time step. }
\end{aligned}
$$


3.1.2.4 Numerical Analysis. The analytical sequence used in this clad swelling model consists primarily of:

(1) Solving for the stability of each nodal point on the cladding using Equation IV-14 and

(2) Modifying the cladding geometry as a function of cladding instability.

Stability of each node is checked by solving Equation IV-14 for the local stability factor $f_{i}$ with $f_{i}<1$ indicating a node with insufficient strength to resist the applied pressure $p$ and $f_{i}>1$ indicating a stable node.

With the assumption that unstable nodes $\left(f_{1}<1\right)$ will deform, the solution process of the swelling model is to specify a deformation for these unstable nodes. Specification of deformations is based on the following:

(1) Nodal deformations are a function of the nodal instability and that node which is the most unstable $\left(F_{m}=\operatorname{maximum} f_{i}\right)$ will deform the most.

(2) The specified displacements must be small enough that adjacent stable nodes are not unrealistically effected.

The process of specifying deformations consists of adding a finite deformation to the nodal deformation calculated during the last time step as

$$
d_{i}=d_{i_{0}}+d_{i}
$$

wliere

$$
\begin{aligned}
& d_{i}=\text { the new deformation of node } i \\
& d_{i_{0}}=\text { the old deformation of node } i \\
& d h_{i}=\quad \text { specified incremental defornation of node } i .
\end{aligned}
$$

The effect of adding an incremented deformation to node $i$ is shown in Figure IV- 10 as decreasing the radius of curvature at i. Examining Equation IV-14 shows this decrease in curvature to increase the stability function $f_{i}$ and thus the local stability at node $i$. An additional effect to be noted from Figure IV-10 is that an increase in deformation at node $i$ causes an increase in curvature at nodes $i+1$ and $i-1$, (possibly to the point of producing negative curvature). An examination of Equation IV-14 shows an increase in curvature to decrease the stability function and thus local cladding stability. The effect, therefore, of locally deforming a weak spot is to strengthen the weak spot but propagate the weakness into the surrounding material, possibly causing additional new instability and further 


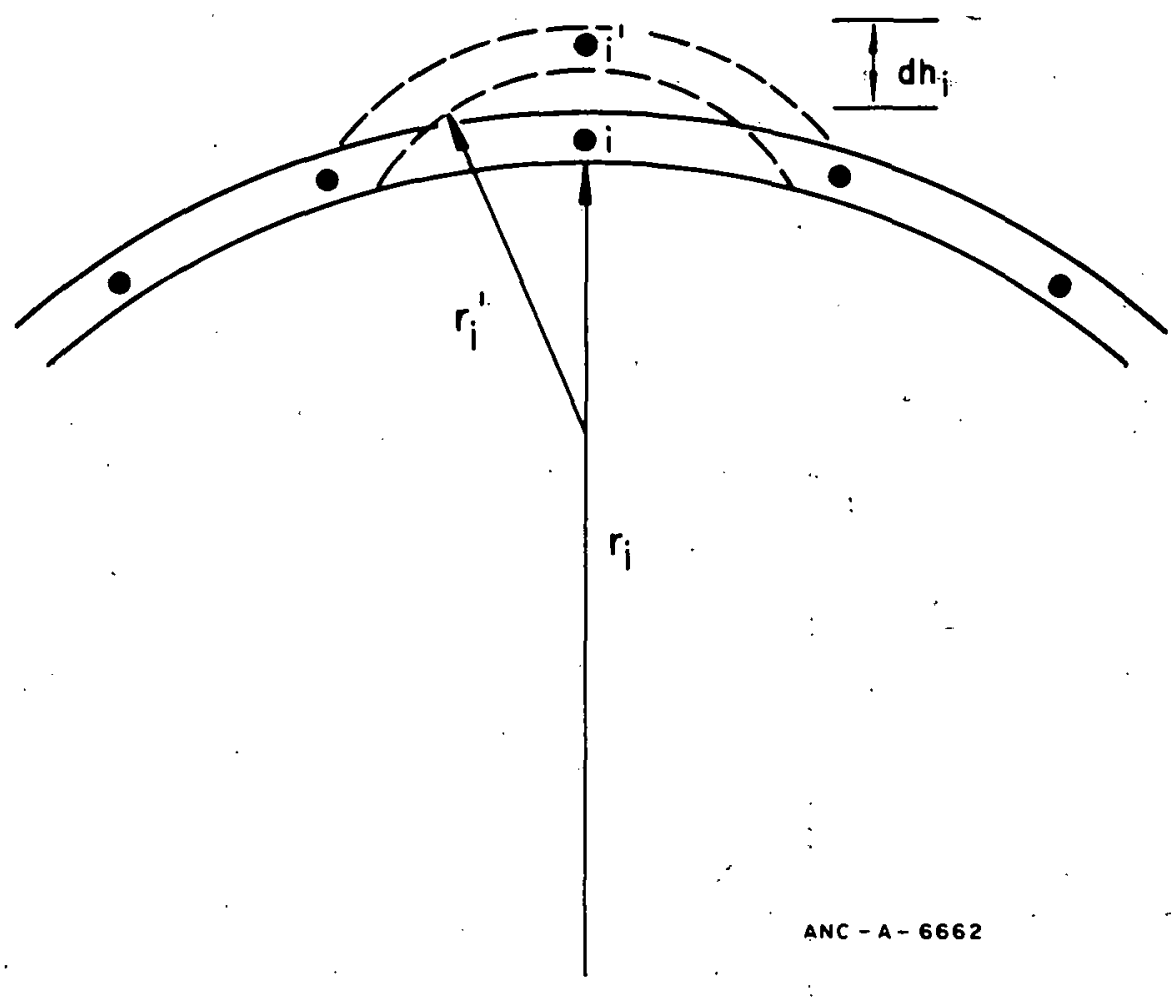

Fig. IV-10 Incremental deformation at node i.

propagation. Careful examination of membrane instabilities such as blisters on tires and ballooning tubes indicate that initial deformation is quite localized and then proceeds to either rupture or to an enlarged stable geometry.

Deformations are specified according to the relation

$$
\mathrm{dh}_{i}=\mathrm{dh}_{\mathrm{m}} \frac{\left(1-\mathrm{f}_{i}\right)^{2}}{1-\mathrm{F}_{\mathrm{m}}}+0.1
$$

where $\mathrm{dh}_{\mathrm{m}}$ is equal to the maximum displacement to be added to any node. A value equal to the cladding thickness has been found to produce a rapid convergence with no apparent numerical or structural instabilities.

Figure IV-1 1 illustrates Equation IV-20 as a function of local instability factors $\mathrm{f}$ and the maximum instability factor $F_{m}$. Figure IV-11 shows the nonlinear aspect of the function in the region near stability $(f=1$ ) which effects less correction as the structure becomes more stable. The 0.1 factor in Equation IV-20 is there to "push" the function past the stability point since corrections very close to stability are very small.

When new nodal displacements are calculated for each node with Equation IV-20, new radii of curvature are calculated as described in 3.1.2.3 above. 


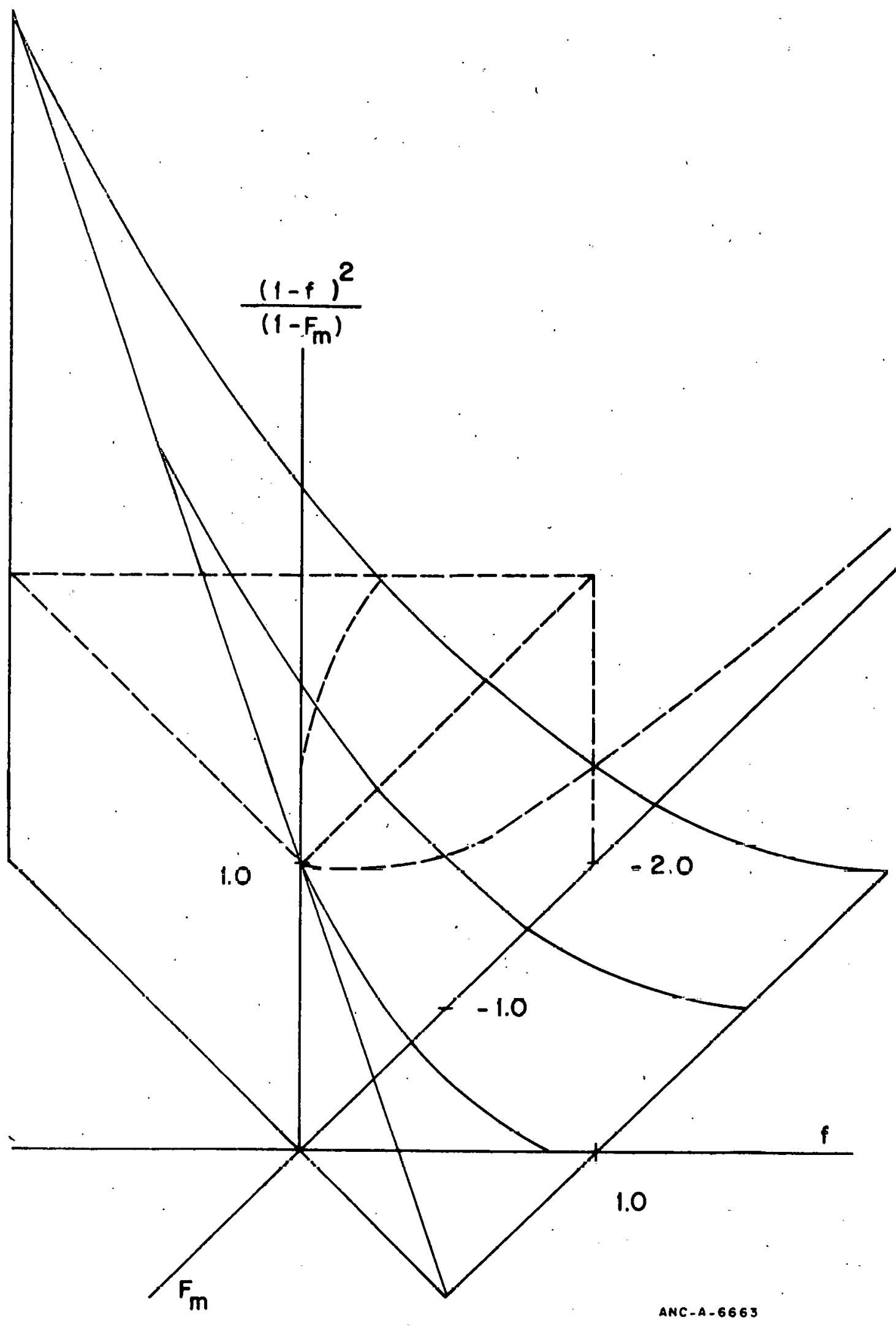

Fig. IV-11 Instability correction factor. 
Stability functions are calculated for each iteration on deformation to account for the effect of deformation on the radii of curvature, cladding thickness, and internal pressure (due to changes in internal volume). Updated values for curvature, cladding thickness, and internal volume are calculated for each iteration by the procedures described in 3.1.2.3. Material yield stress, $\sigma_{y}$, can be modified at each iteration to consider strain rate and temperature effects on yield stress. Calculation of temperature and strain rate effects are covered in 3.1.2.5 and 3.1.2.6, respectively. Figure IV-12 is a flow chart of the numerical analysis procedure for the swelling model.

\subsubsection{Conduction Model}

(1) Expanding Clad Temperature Effects. As the cladding expands away from the hot fuel pellet surface, the cladding temperature will change under the combined effects of (a) decreased gap conductance from increasing gap thickness, (b) increased surface cooling due to increased area, (c) increased surface cooling due to local flow phenomena, (d) increased fuel surface temperature to decreased gap conductance, and (e) heat capacitance of cladding.

The model formulated to include these combined effects considers the cladding temperature as a function of the local power or surface heat flux value. Figure IV-13 illustrates the major assumption of the model which is that the heat flux $q$ from an area of the fuel $A_{f}$ is transferred through a corresponding area of the cladding $A_{c l}$, throughout the transient. Considering this model and assumption, the temperature of the cladding is governed by

$$
\rho \mathrm{p}_{\mathrm{cl}}^{\mathrm{V}} \frac{\partial \mathrm{T}_{\mathrm{cl}}}{\partial \mathrm{t}}=\ddot{\mathrm{qA}}_{\mathrm{f}}+\mathrm{A}_{\mathrm{cl}} \mathrm{h}_{\mathrm{s}}\left(\mathrm{T}_{\mathrm{B}}-\mathrm{T}_{\mathrm{cl}}\right)
$$

where

$$
\begin{aligned}
& \mathrm{C}_{\mathrm{p}}=\text { cladding heat capacity } \\
& \rho \quad=\quad \text { cladding density } \\
& \mathrm{V}_{\mathrm{cl}}=\quad \text { cladding nodai volume } \\
& \mathrm{T}_{\mathrm{cl}}=\text { cladding average temperature } \\
& \mathrm{T}_{\mathrm{B}}=\text { bulk coolant temperature } \\
& \mathrm{h}_{\mathrm{s}}=\text { cladding surface heat transfer coefficient (see following section) } \\
& \mathrm{A}_{\mathrm{cl}}=\text { cladding nodal area } \\
& \mathrm{A}_{\mathrm{f}} \quad=\quad \text { fuel nodal area } \\
& \ddot{q} \quad=\quad \text { fuel surface heat flux } \\
& \mathrm{t}=\text { time. }
\end{aligned}
$$




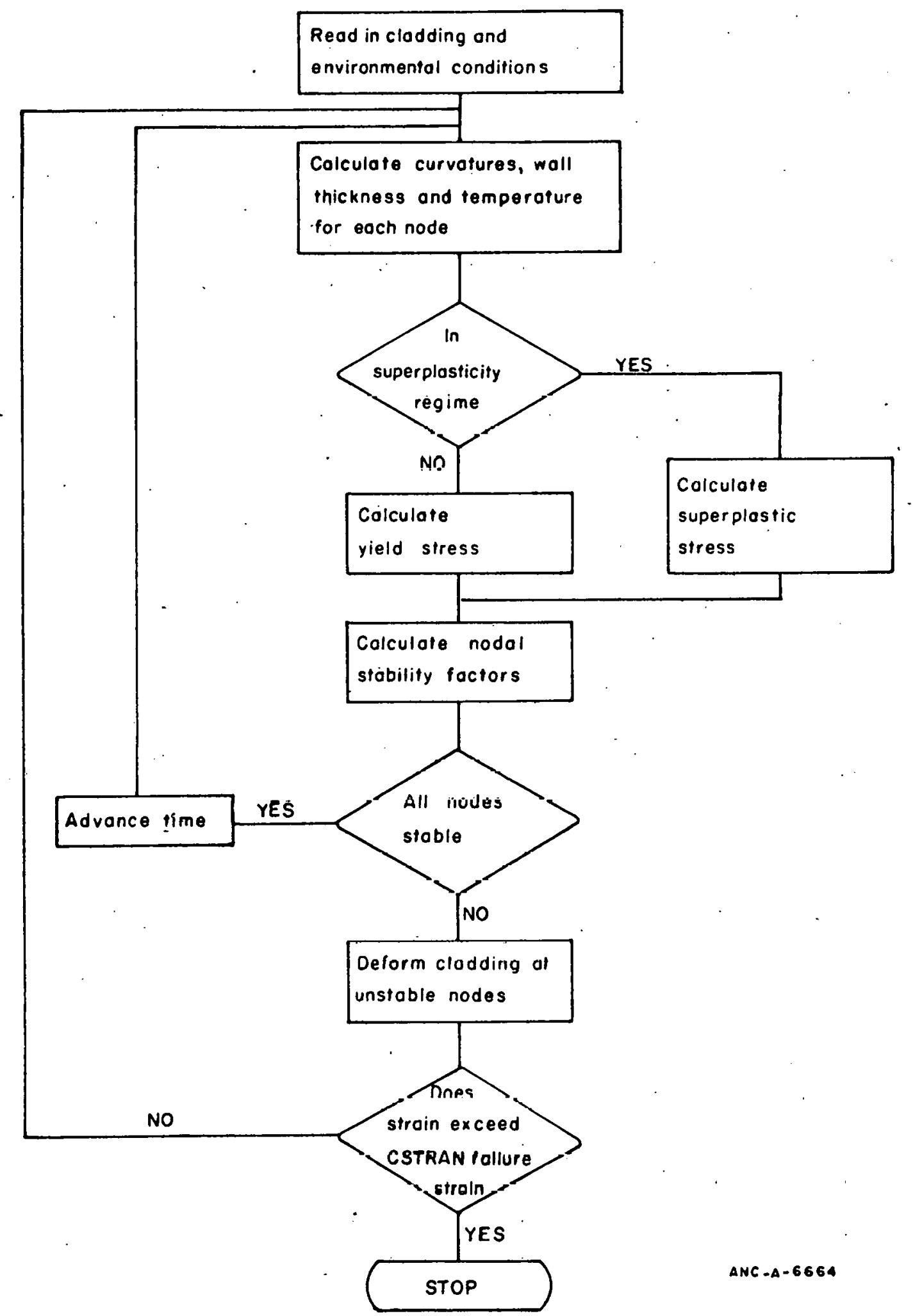

Fig. IV-12 BALLOON flow diagram. 


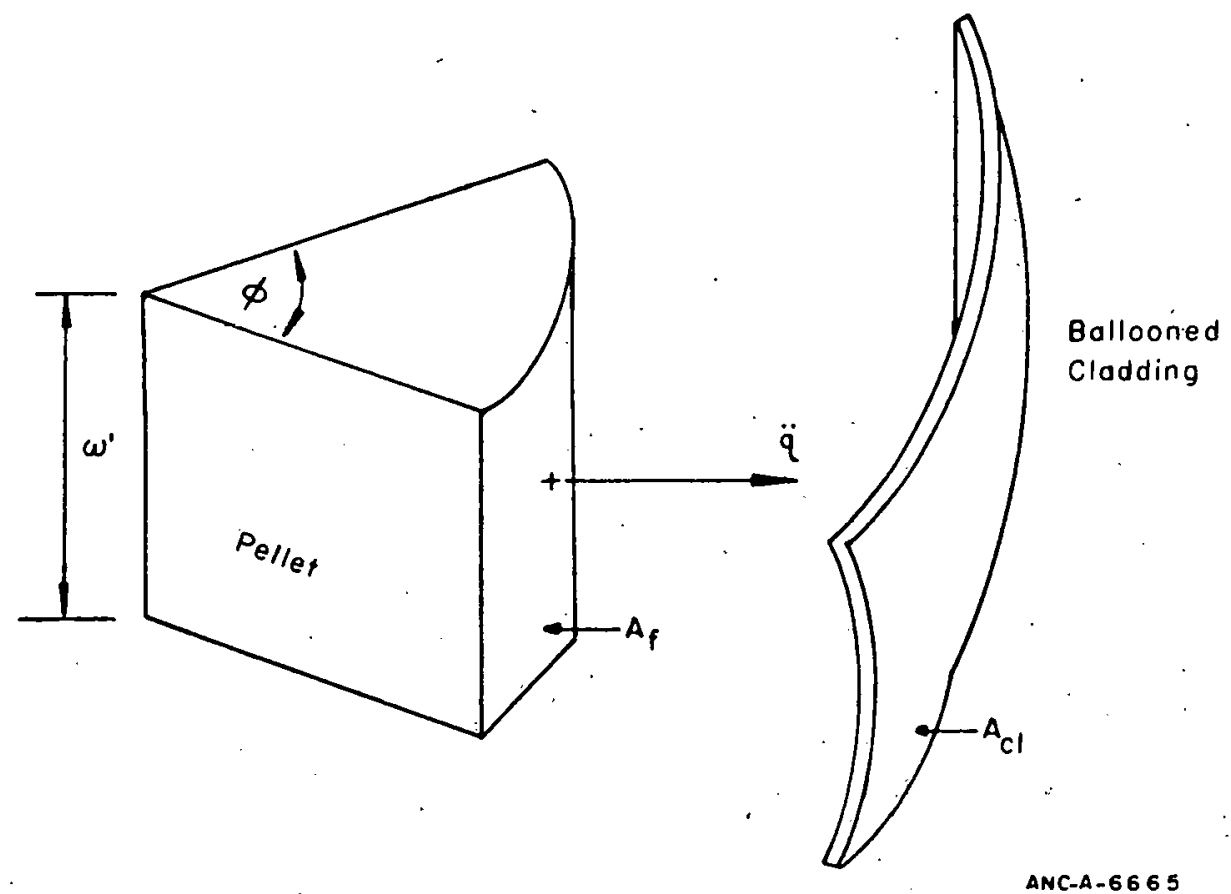

Fig. IV-1 3 Energy transfer during ballooning.

Solution of Equation IV-21 for the time dependent cladding temperature gives

$$
\mathrm{T}_{C 1}=\left(\mathrm{T}_{0}-\mathrm{B} / \mathrm{A}\right) \mathrm{e}^{-\mathrm{At}}+\mathrm{B} / \mathrm{A}
$$

where

$$
\begin{aligned}
& \mathrm{T}_{\mathrm{O}} \quad=\quad \text { cladding inital temperature } \\
& \mathrm{A}=\mathrm{A}_{\mathrm{cl}} /\left(\rho \mathrm{C}_{\mathrm{p}} \mathrm{V}_{\mathrm{cl}}\right)
\end{aligned}
$$

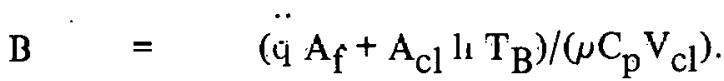

(2) Local Flow Effects. Additional clad cooling will result as the cladding swells into the coolant channel. This additional cooling is modeled as an increase in the surface heat transfer coefficient by the relation

$$
h_{s}=h_{0}\left(1+c_{1} d_{i / r_{0}}\right)
$$

where

$$
\begin{aligned}
& \mathrm{h}_{\mathrm{s}}=\quad \text { cladding transfer coefficient for expanded cladding } \\
& \mathrm{h}_{\mathrm{O}}=\text { cladding heat transfer coefficient calculated by FRAP-T }
\end{aligned}
$$




$$
\begin{array}{ll}
\mathrm{d}_{\mathrm{i}} & =\text { radial location of } \mathrm{i}^{\text {th }} \text { node } \\
\mathrm{r}_{\mathrm{O}} & =\quad \text { initial radius of } \mathrm{i}^{\text {th }} \text { node } \\
\mathrm{C}_{1} & =\text { heat transfer factor (user specified). }
\end{array}
$$

3.1.2.6 Cladding Strength Model. Cladding stress is calculated as a function of temperature, strain, and strain rate. The models used for cladding stress represent state of the art formulations, such as CSTRAN, and data from experimental programs [IV8, IV-9, IV-10] In the case of models describing the strain rate dependence of zircaloy 4 , only enough data[IV-11,IV-12,IV-13] exists to formulate a generic type model, but the model is one which can be used for parametric studies.

The material stress model used in BALLOON is defined in two regions: (a) the normal plasticity region where stress and strain can be related as a function of temperature using a power law formulation and (b) the superplastic region where strain rate effects are considered. The individual models and their interface are described.

Normal Plastic Behavior. In the region of normal plastic behavior, the equilibrium stress used in Equation IV-14 for determination of stability is set equal to the material yield stress at the local temperature. The yield stress is calculated as the intersection of the elastic portion of the stress-strain curve and the nonlinear portion of the stress strain curve as calculated by a power law.

Superplastic Behavior. Superplastic behavior is considered in the region of strain rates from $10^{-7} \mathrm{sec}^{-1}$ to $10^{-1} \mathrm{sec}^{-1}$ and for temperature from $750^{\circ} \mathrm{C}$ to $1050^{\circ} \mathrm{C}$. The response of the cladding in this regime is modeled considering the basic effect of superplasticity, i.e., that increased 'strain rate within certain response regimes causes an increase in stress. Figure IV-14 illustrates the stress $\delta$ vs. strain rate $\epsilon$ effect. The slope $m$ of the curve in Figure IV-14 is an indication of the strain rate effect and is defined as

$$
\mathrm{m}=\frac{\mathrm{d} \log \sigma}{\mathrm{d} \operatorname{lng} \dot{\mathrm{s}}}
$$

Values of $m$ have been obtained for many materials [IV-11]. but data for ziracloy-4 is still sparse. 'l'he model reported herein is based on a fit of sómé initial dáta for $m$ and is considered preliminary.

Equation IV-24 for $m$ is rewnitten to calculate the local slope $m$ as

$$
m=\frac{\log \sigma_{i}-\log \sigma_{i-1}}{\log \dot{\varepsilon}_{i}-\log \dot{\varepsilon}_{i-1}}
$$




$$
=
$$


where

$$
\begin{array}{ll}
\mathrm{i} & =\mathrm{i}^{\text {th }} \text { time step } \\
\mathrm{i}-1 & =\text { previous time step. }
\end{array}
$$

Equation IV-25 can be simplified and rearranged to give

$$
\sigma_{i}=\sigma_{i-1}\left(\frac{\dot{\varepsilon}_{i}}{\dot{\varepsilon}_{i-1}}\right)^{\mathrm{m}}
$$

Equation IV-26 is the basis of the strain-rate-dependent, superplasticity model used by BALLOON. Several features of Equation IV-26 are:

(1) The stress at a new time step is a function of the stress level at the previous time step.

(2) If the strain rate is constant between time steps, even for a high strain rate, the stress will remain constant.

(3) The model is reversible. If loading is decreased and the strain rate decreases, the stress at new time steps will be decreased.

The model calculates the stress, $\sigma_{i}$, by one of the following procedures:

(1) If the strain rate and local temperature are outside the range of superplasticity, $\sigma_{i}$ is calculated as the yleld stress of the material.

(2) If the strain rate and local temperature are within the range of superplasticity, Equation IV-26 is solved for the stress at the new time step. If the material has just become superplastic, the yield stress from the previous time step is taken as $\sigma_{i-1}$.

\subsection{BALLOON Model Comparison Results}

Results are presented in the form of comparison with experimental data and parametric studies. In most of the accident conditions for which this swelling model is developed, there is little or no data to support assumptions and compare results. Planned experiments such as LOFT, PBF, and ongoing RSR task will provide further data with which to compare and update this model. 
3.2.1 Comparison with Experimental Data. Data have been obtained on the ballooning response of reactor cladding during thermal transients $[$ IV $-8,-9,-10]$, but the data are incomplete with regard to the requirements of the model described in this report. Some of the noted shortcomings of the available data are a lack of information on:

\section{(1) Local temperature distribution}

(2) Time dependent spatial motion during swclling

(3) Surface heat transfer to coolant (most tests were run in a stagnant atmosphére and are not helpful in evaluating the response calculated using the conduction models described in 3.1.2.5)

(4) Internal pressure

(5) Local strain, strain rate, and time of local instabilities leading to clad rupture.

The experimental data reported herein and compared with analytical results were obtained from burst experiments made at the Idaho National Engineering Laboratory (INEL) in connection with the Irradiation Simulation Study (ISS). The burst tests consisted of 6-inch sections of pressurized zircaloy tubing heated with an induction furnace until the cladding swelled and ruptured. Test tubes were heated over the 2 -inch center section on a temperature ramp typical of the curve in Figure IV-15. This transient temperature profile was predicted by FRAP-T for a typical LOCA and was used as a base case for the ISS. Pressure was controlled during the test and varied as shown in Figure IV-15. The pressure history of Figure IV-15 was also predicted by FRAP-T as part of the LOCA analysis mentioned above.

Figure IV-16 shows the comparison between deflected shapes as traced from the deformed cladding and as predicted by the BALLOON model. The shapes of the experimentally deformed cladding in Figure IV-16 were obtained by laying the test specimen on a piece of paper, with points of maximum deflection toward the top of the page, and tracing the deformed shape. Perforation of the cladding is always at the point of maximum deflection. Discontinuities in the experimental shapes (HR-2) appear because some perforations were so large and the metal around the hole so jagged that a realistic shape could not be defined at the point of rupture.

Failure in the model was taken as a local strain level of $100 \%$. This value of strain at failure is consistent with several data sources $[$ IV-8, IV-9, IV-10] for failure strain at $2,000^{\circ} \mathrm{F}$. The failure strain data as presented in Figure IV-17 is a function of total circumferential elongation (TCE) but, assuming symmetric swelling of the data source test specimens, the TCE data can be used as local failure strain data and applied to a nonsymmetric swelling model such as BALLOON. 

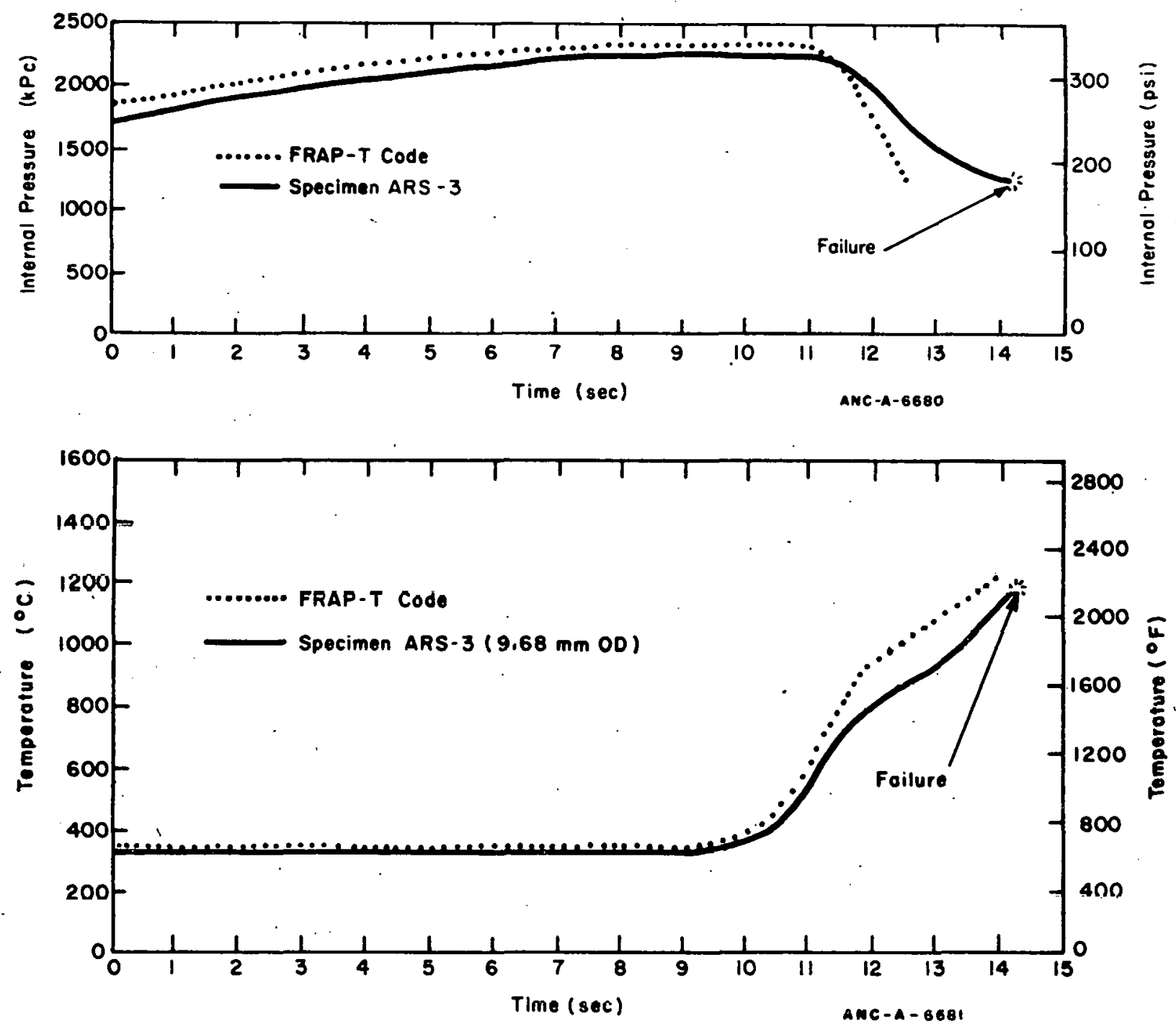

Fig. IV-15 Pressure and temperature history of burst samples.

3.2.2 Parametric Studies. Parametric studies were made to check sensitivity of the overall swelling model to certain of the assumptions and submodels of the formulation.

Nodal Spacing. In most analytical formulations such as finite element and finite difference solutions, the smaller the nodal spacing the more accurate the solution. The axial and circumferential spacing of element nodes was varied and the influence of this spacing on maximum displacement recorded. The effect of nodal spacing is shown in Figures IV-18 and IV-19.

Local Cladding Weakness. It is assumed that fuel cladding starts to balloon because of a local weak spot in the cladding. This relative weakness in the cladding can be due to local thinning, a manufacturing flaw, or just a hot spot which reduces the local cladding strength. 


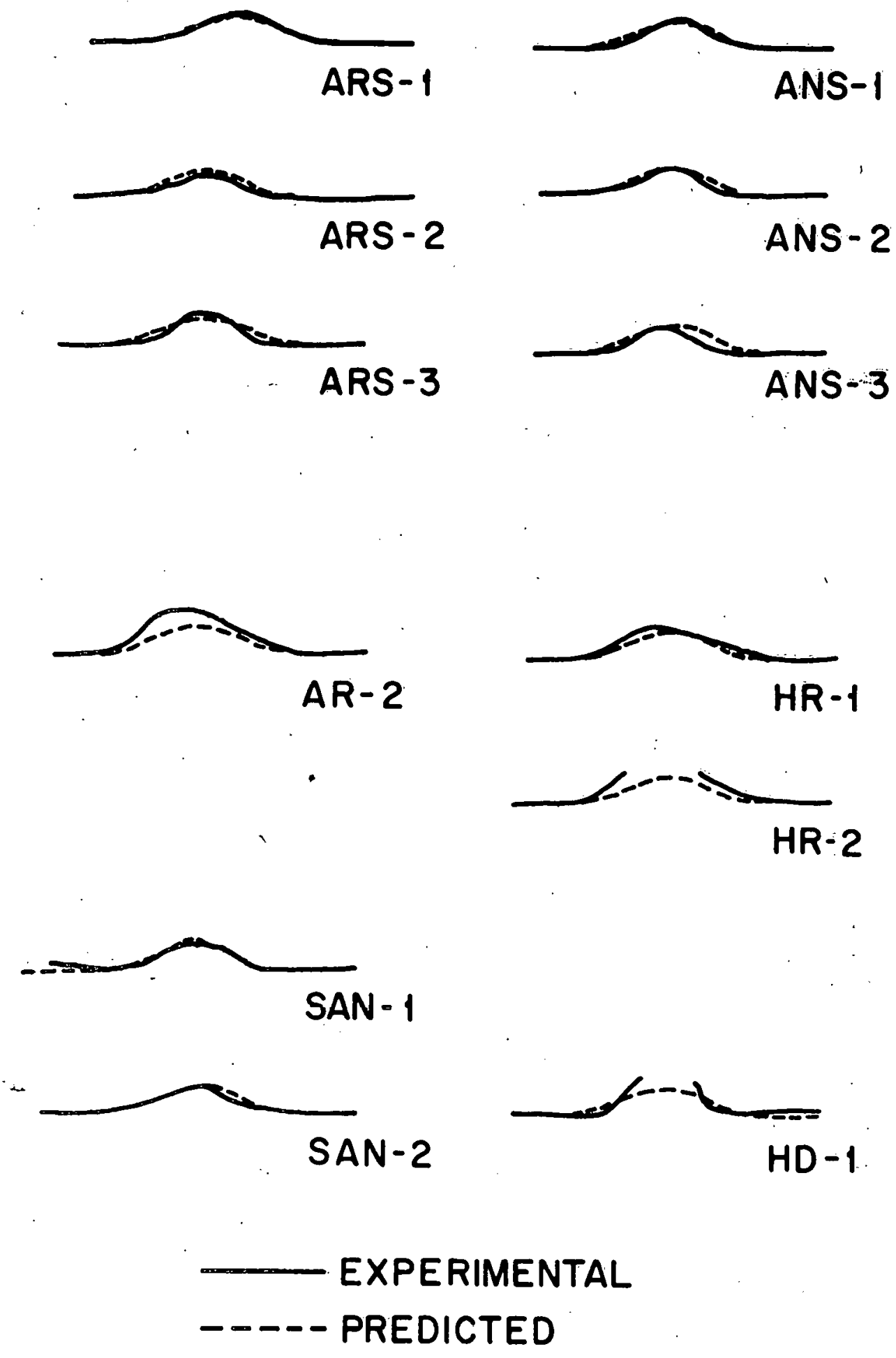

Fig. IV-16 Experimental vs. predicted ballooning shapes. 


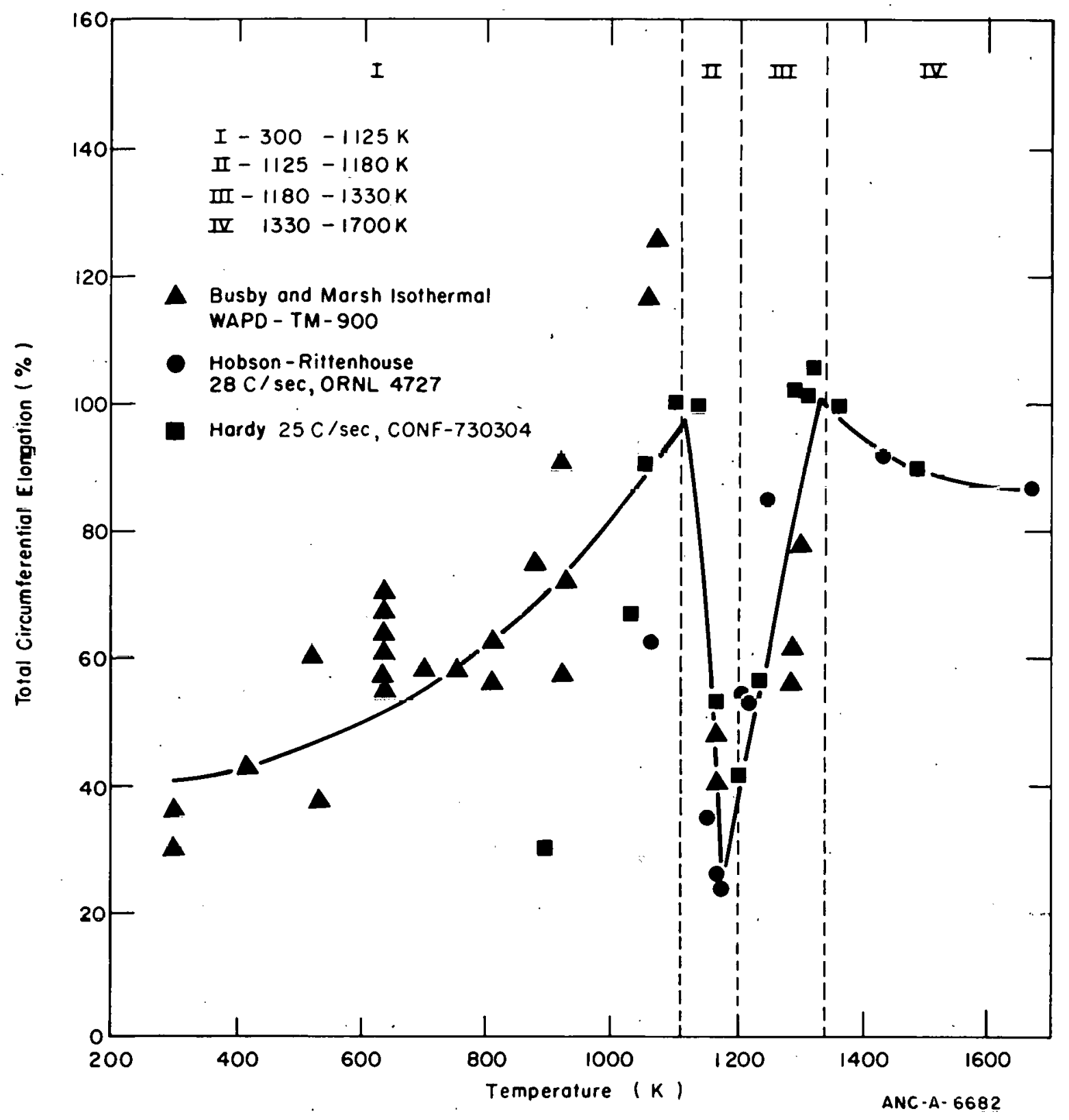

Fig IV-17 Tntal circumferential elnngation (TCF) as a function of temperature.

The result is the same, and given a threshold instability loading condition, the weak spot will deform first.

A series of parametric analyses were made with the BALLOON model in which the relative strength of the weak spot varied. The effect of this variance on other parameters was calculated and is shown in Figure IV-20. As stated above, local weak spots (or points of increased resistance) can be temperature related (local cooling can strengthen the cladding). In order to keep the spatial and time dependence thermal effects out of the parametric study, the local cladding cooling model described in 3.1.2.5 under "Local Flow Effects" was "turned off" and a constant temperature increase was used. 


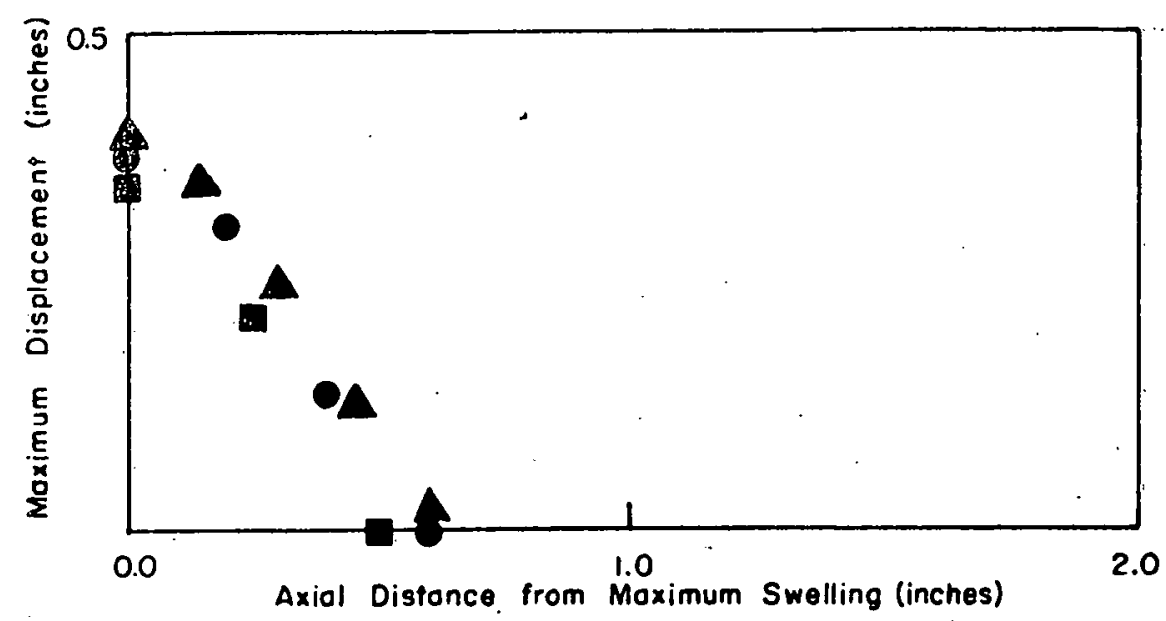

$\omega^{\prime}=0.20$ inch
$\omega^{\prime}=0.25$ inch
$\omega^{\prime}=0.15$ inch

Circumferential Spocing $=\pi / 7$ rod

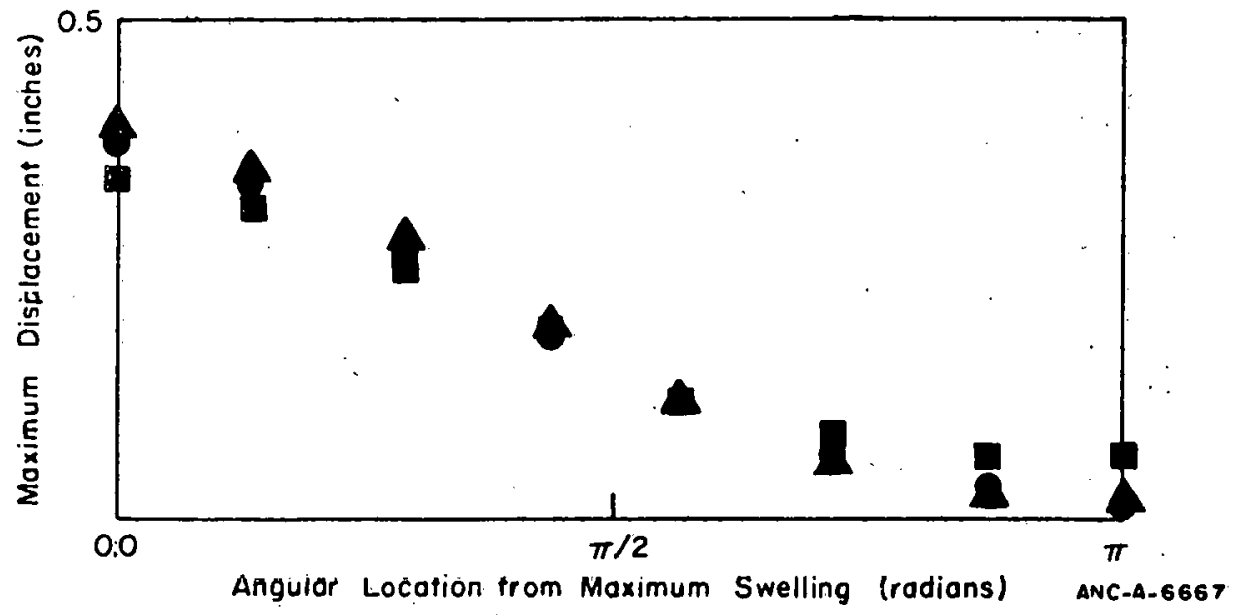

Fig. IV-18 Effect of axial spacing on cladding displacement. 


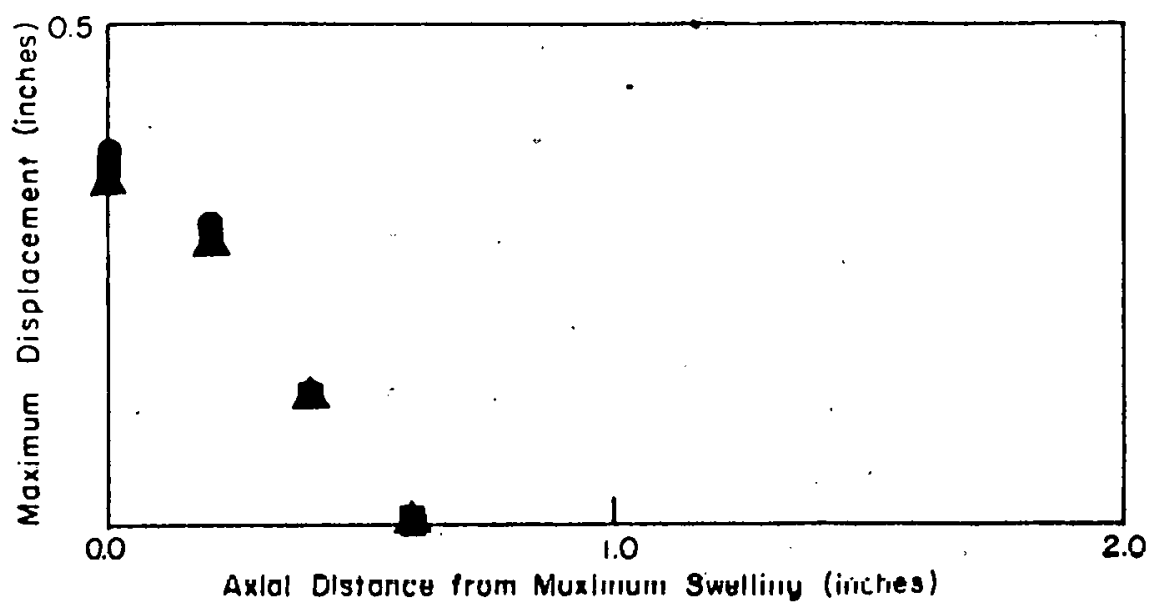

$\phi=\pi / 7$ rodians
$\phi=\pi / 5$ rodians
$\Delta \phi=\pi / 9$ rodians

Axiol Spocing $=0.2$ inch

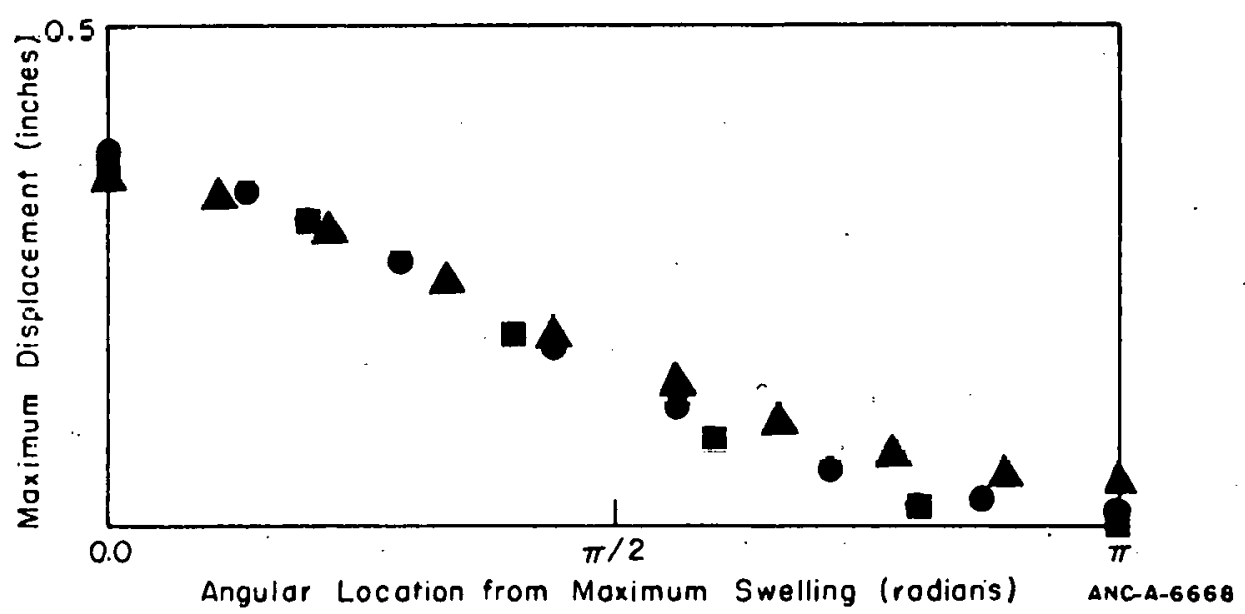

Fig. IV-19 Effect of circumferential spacing on cladding displacement. 


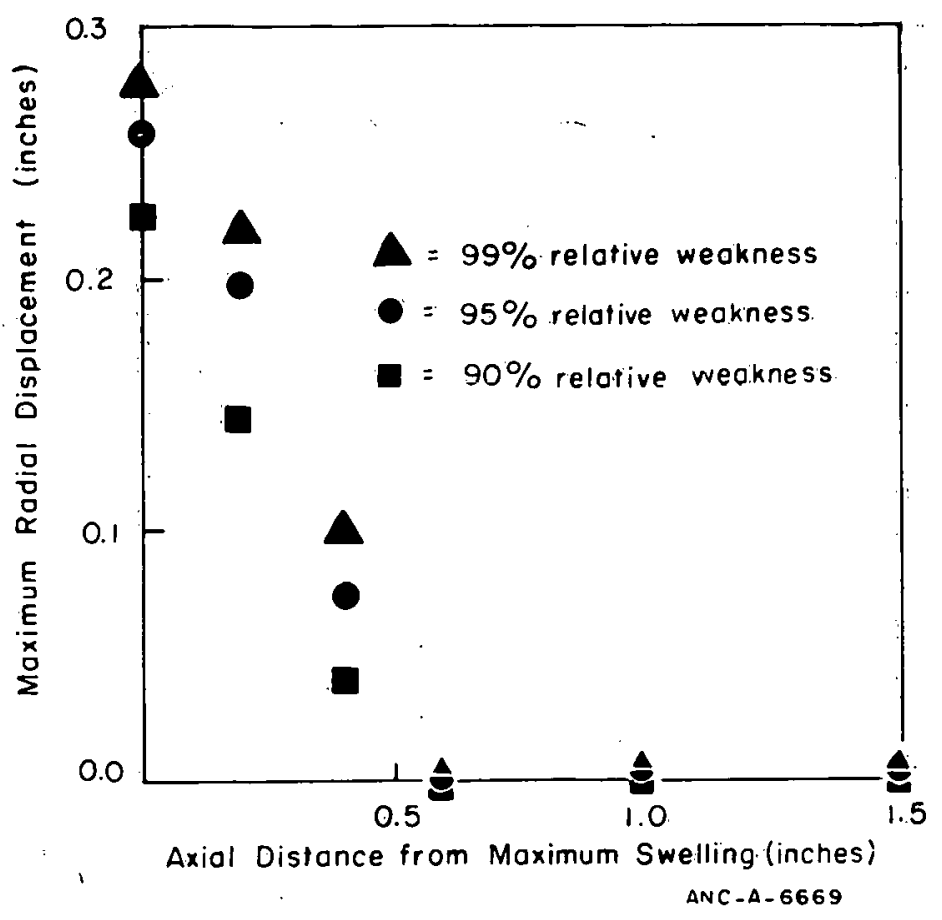

Fig. IV-20 Effect of relative strength of central node on maximum swelling.

Strain at Failure. Analyses were performed with the BALLOON model over a range of clad thickness and internal pressure values designed to vary the temperature, and thus the strain, at failure. Figure IV-21 illustrates a portion of the TCE failure curve of Figure IV-17 from $1400^{\circ} \mathrm{F}$ to $2200^{\circ} \mathrm{F}$. Shown in Figure IV-21 are the deformation histories of cladding under different conditions. Table IV-I gives the baseline data for the cladding analyses. Variations in the baseline parameters are noted in Figure IV-21. Analysis results noted in two spots by the same number represent the notation of maximum cladding temperature (at the boundary) and the minimum temperature (at the point of maximum swelling). Failure is predicted when the strain at minimum temperature exceeds the failure criteria.

Several things are to he noted from Figure.IV-21:

(1) The temperature at which failure is predicted to occur, i.e., " maximum, minimum, or average cladding temperature, can make a substantial difference in the predicted swelling.

(2) The temperature at which initial instability occurs is very important. If initial instability occurs at $1,500^{\circ} \mathrm{F}$ to $1,600^{\circ} \mathrm{F}$, the failure has a high probability of occurring at a low total strain in the $\alpha-\beta$ regime. Instability temperature higher than $1,700^{\circ} \mathrm{F}$ have a high probability of resulting in large total strains at failure.

(3) The temperature rise rate (and thus the loss of strength rate) and the change in internal pressure due to swelling influences the rate 


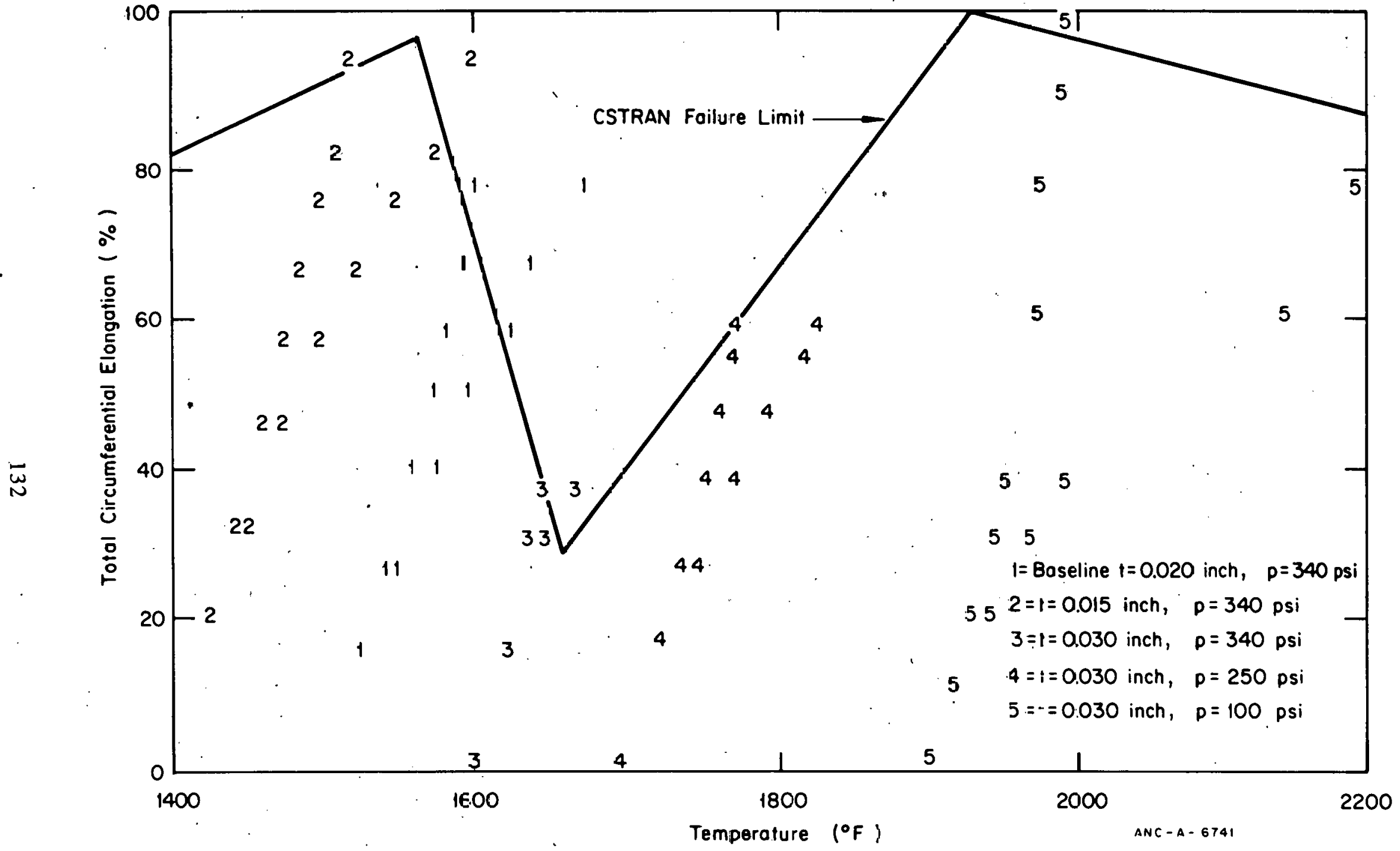

Fig: IV-21 Influence of parameters on strain at failure. 


\begin{tabular}{lll}
\hline Wall thickness & $t$ & $=0.020 \mathrm{inch}$ \\
Initial radius & $\mathrm{r}_{0}=0.188 \mathrm{inch}$ \\
Pressure difference & $\mathrm{p}=340 \mathrm{psi}$ \\
Axial nodal spacing & $\omega^{\prime}=0.2 \mathrm{inch}$ \\
Circumferential nodal spacing & $\phi^{\prime}=\pi / 7 \mathrm{radians}$ \\
Incremental displacement & $\mathrm{dh}_{\max }=0.02 \mathrm{inch}$ \\
Surface heat transfer & $\mathrm{h}_{\mathrm{o}}=100 \mathrm{Btu} / \mathrm{hr} \mathrm{ft}^{2}$ \\
Decay power flux & $\ddot{\mathrm{q}}^{2}=33,300 \mathrm{Btu} / \mathrm{hr}^{2}$ \\
Relative strength of center node & $\rho^{\omega}=0.95$ \\
\hline
\end{tabular}

at which swelling occurs. Due to the rapid change in failure strain versus temperature in certain regions, a slight change in the ballooning rate can greatly influence strain at failure.

(4) The local cooling effect can cause a large difference between the maximum, minimum, and average cladding temperature. This large difference makes it difficult to logically define failure as a function of failure data specified in terms of a single temperature.

(5) Any parameter which affects the instability temperature or rate of change of temperature with respect to strain is important in determining strain at failure. These parameters include cladding thickness, gap conductances, internal gas flow rates, and strain rate effects.

Strain Rate Effects. Evaluation of the strain rate model in BALLOON has not been sufficient to draw conclusions. The model is working in a preliminary version and toes produce results consistent with present understanding of the data. The data, however, do not extend into the regime where ballooning cladding has been observed to occur, i.e., at strain rate of from $10^{0} \mathrm{sec}^{-1}$ to $10^{1} \mathrm{sec}^{-1}$. Development and evaluation of the superplasticity model is continuing.

\subsection{BALLON Model Development Conclusions}

A model has been developed which calculates the deformation of reactor fuel cladding as a function of realistic environmental conditions. The major response phenomena such as temperature dependent material properties, variable boundary conditions, and geometric 
strengthening of the cladding are considered. The model has been shown to predict the shape of experimentally deformed cladding using a first-principle type formulation of the governing equations.

The model has been used to show deficiencies in the available data based on cladding ballooning in that no data now exists on spatial temperature variation on the cladding or time dependent local strain. Data of this type are required to further check the adequacy of the model described here.

Data on cladding strain rate effects are needed for strain rates up to $10^{1} \mathrm{sec}^{-1}$ for use in predicting the type of cladding response already observed in experiments.

Local cooling of the cladding can be very important in the calculation of total swelling (for blockage calculations) and rupture (for fission gas release).

\section{FUEL BEIIAVIOR VERIFIC $\Lambda$ TION}

\subsection{Cladding Axial Growth}

D. L. Hagrman

A model has been developed for calculating the fractional change in length of zircaloy tubes due solely to irradiation-induced growth. The effects of fast neutron fluence, tubing texture, cladding temperature, and cold work are included in the model. This model applies equally well to zircaloy- 2 and zircaloy -4 . The model will be incorporated in a future version of MATPRO, the Material Properties subroutine of FRAP-T.

4.1.1 Summary. The following equation has been developed to model the irradiationinduced growth of zircaloy tubes at temperatures between 40 and $360^{\circ} \mathrm{C}$ (applying to the normal range for cladding temperatures in LWRs):

$$
\frac{\Delta L}{L}=A\left[\exp \left(T_{0} / T\right)\right](\phi t)^{1 / 2}\left(1-3 E_{z}\right)(1+D * C W)
$$

where

$$
\begin{array}{ll}
\frac{\Delta \mathrm{L}}{\mathrm{L}} & =\quad \text { fractional change in length due to growth } \\
\mathrm{A} & \left.=1.407 \times 10^{-16} \text { (neutrons } / \mathrm{m}^{2}\right)^{-1 / 2} \\
\mathrm{~T}_{\mathrm{O}} & =240.8 \mathrm{~K} \\
\mathrm{~T} & =\text { cladding temperature }(\mathrm{K}) \\
\phi & =\text { fast neutron flux }\left(\mathrm{n} / \mathrm{m}^{2}-\mathrm{sec}\right)(\mathrm{E}>1.0 \mathrm{MeV})
\end{array}
$$




$$
\begin{array}{ll}
\mathrm{t} & =\text { time }(\mathrm{sec}) \\
\mathrm{f}_{\mathrm{Z}} & =\text { texture factor }[\mathrm{a}] \text { for the tubing axis } \\
\mathrm{CW} & =\text { cold work (fraction of cross-sectional area reduction) } \\
\mathrm{D} & =2.0 \text { (unitless). }
\end{array}
$$

A comparison of values calculated using the growth model with experimental results is presented in Figure IV-22. Comparison with the data shown from cold worked tubes was not possible because the amount of cold work was not reported. The dependence of the cold worked tube growth on the square root of fluence has been tested, however, as discussed in Section 4.1.2.

4.1.2 Background and Approach. The amount of usable data which is available for constructing and testing an irradiation growth model is limited by the difficulty of separating changes due to irradiation growth from changes in length due to both fuel-cladding mechanical interaction and to creep. Even in cases where mechanical interactions are small because of large fuel-cladding gaps, the effect of creep remains. Isolation of the creep contribution requires knowledge of the effects of tubing texture. Moreover, texture effects are important in considering the results of some experimental techniques which rely upon measuring the difference between circumferential and axial changes in length. For these reasons the effects of texture were considered first and the data was normalized to a standard texture $\left(\mathrm{f}_{\mathrm{z}}=0.05\right)$ before considering other effects on axial growth. The model was developed further by sequentially removing the effects of different parameters from the data so that the remaining phenomena could be isolated and modeled.

Fast neutron flux and temperature both affect the growth rate by varying the concentration of interstitials which are free to migrate and cause growth. Since theoretical considerations imply a complex relation between the temperature, fast neutron flux, timè, and the rate of growth, an empirical approach was used to approximate the limited data even though the empirical model suggests unrealistic interstitial migration energies. It should he noted that this model employs only the product of flux and time (fluence), although the flux used to achieve a given fluence does have theoretical significance (see Reference IV-14).

An empirical approach was also used to model the effect of cold work on zircaloy tube growth. The limited data was fit using an independent factor of the form $(1+$ constant $\mathrm{x}$ cold work) - the least complex form consistent with the preliminary data reported to date. The main conclusion from the available data is that cold work increases the rate of growth at low fluence. At higher fluences, the growth rate of annealed tubing may decrease rapidly. Cold worked tubing continues to grow at higher fluences at nearly the rate established during early irradiation.

[a] $f_{z}$ is the effective fraction of cells aligned with their $<0001>$ (or $c$ ) axis parallel to the lubing axis as detcrmince by X-ray diffraction analysis, $A$ value of $f_{z}=0.05$ is typical ${ }^{[I V-15]}$. 


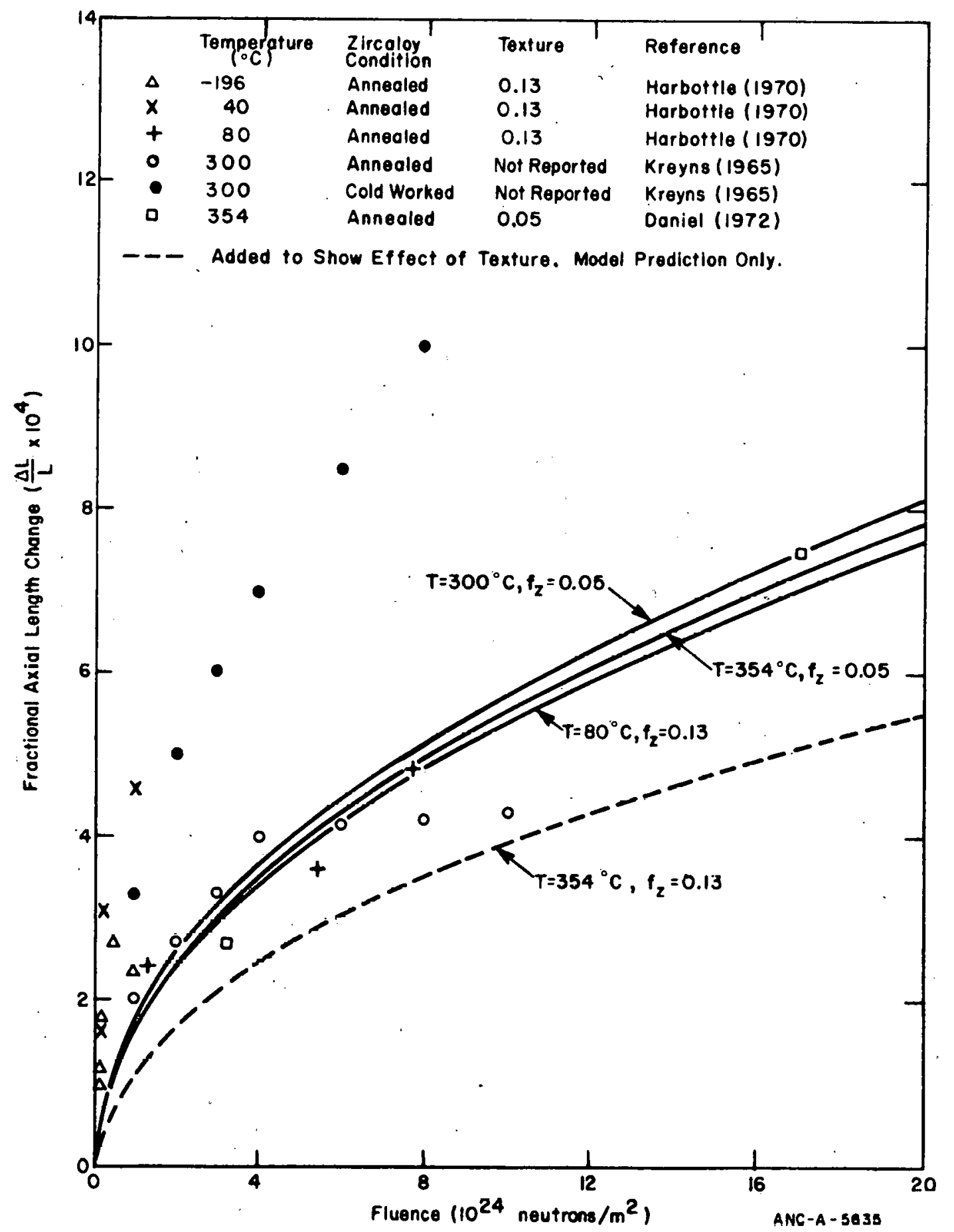

Fig. IV-22 Model predictions and measured values of zircaloy tube axial growth as a function of fast neutron fluence, irradiation temperature, cold work, and texture coefficient $\mathrm{f}_{\mathrm{z}}$.

4.1.3 Review of Experimental Data. Samples of zirconium, zircaloy-2, and zircaloy-4 irradiated in a fast neutron flux (E $>1 \mathrm{MeV})$ to fluences of $10^{25} \mathrm{n} / \mathrm{m}^{2}$ show typical axial growth on the order of $0.1 \%$ of length or less. Since the effects of fuel-cladding mechanical interactions and pressure differentials across the cladding compete with the smaller effects of irradiation growth, the relatively plentiful data on the in-pile change in length of fuel elements [IV-16,IV-17,IV-18] are not directly useful in determining the change in cladding 
length: due to irradiation growth. Data: on: thimble tubes: or: othery structural: elements relatively free of confounding effects would be useful but:none have been published: Tàble: IV.-II summarizes the data which have been used in the present model development.

TABLE IV:-II

MEASUREMENTS OF: GROWTH. IN ZIRCALOY TUBING.

\begin{tabular}{|c|c|c|c|c|c|c|}
\hline Source & $\begin{array}{l}\Delta \mathrm{L} / \mathrm{L} \\
\left(10^{-4}\right)\end{array}$ & $\begin{array}{l}\text { D1fferential }{ }^{[a]} \\
\Delta \mathrm{L} / \mathrm{L}\left(10^{-4}\right) \\
\end{array}$ & $\begin{array}{c}\begin{array}{c}\text { Fast } \\
\text { F1uence } \\
\left(10^{2} 2 / \mathrm{m}^{2}\right) \\
\end{array} \\
\end{array}$ & Material & $\begin{array}{c}\text { Fast Flux. } \\
\left(1.0^{17} \mathrm{n} / \mathrm{m}^{2} \mathrm{sec}\right) \\
\end{array}$ & $\begin{array}{l}\text { Irradiated } \\
\text { Temperature } \\
\text { (c) } \\
\end{array}$ \\
\hline \multirow[t]{13}{*}{ Kreyns $[$ IV-19] } & 2 & & 100 & Annealed zircaloy- 4 & $(?)$ & 300 \\
\hline & 2.7 & & 200 & & & \\
\hline & 3.3 & & 300 & & & \\
\hline & 4.0 & & 400 & & & \\
\hline & 4.15 & & 600 & & & \\
\hline & 4.2 & & 800 & & & \\
\hline & 4.3 & & 1,000 & & & \\
\hline & 3.3 & & 100 & Cold worked zircaloy- 4 & (?) & 300 \\
\hline & 5. & & 200 & & & \\
\hline & 6. & & 300 & & & \\
\hline & 7 & $\cdot$ & 400 & & & \\
\hline & 8.5 & & 600 & $\cdots: \cdots$ & & , \\
\hline & 10 & & 800 & & & \\
\hline \multirow[t]{2}{*}{ Daniel $[$ IV-15,-20] } & 2.7 & & 310 & Annealed zircaloy -4 & 12.5 & 354 \\
\hline & 7.5 & & 1,700 & & & \\
\hline \multirow[t]{11}{*}{ Harbottle $e^{[I V-14]}$} & & $1.2 \pm 0.2$ & 4.99 & Annealed zlrcaloy-2 & 3 & -196 \\
\hline & & $1.5 \pm 0.3$ & 9.7 & & & $\cdot$ \\
\hline & & $2.3 \pm 0.3$ & 19 & & & \\
\hline & & $3.5 \pm 0.5$ & 50 & & & \\
\hline & & $3.0 \pm 0.1$ & 98 & & & \\
\hline & & $2.1 \pm 0.2$ & 8.2 & Annealed zircaloy-2 & 3 & 40 \\
\hline & & $4.0 \pm 0.2$ & 29 & & & \\
\hline & & $5.6 \pm 0.4$ & 100 & & & \\
\hline & & $3.1 \pm 0.4$ & 130. & Annealed z1rcaloy-2 & 1.2 & 80 \\
\hline & & $4.7 \pm 0.4$ & 540 & & & \\
\hline & & $6.3 \pm 1.0$ & 770 & & & \\
\hline
\end{tabular}

[a]. Only thè difference between Iongltudinal and transfer changes in length was reported. 
Early data on the irradiation-induced axial growth of zircaloy 4 tubing at $300^{\circ} \mathrm{C}$ was obtained by Kreyns ${ }^{[I V-19]}$. His experiments indicated that growth of cold worked tube is proportional to the square root of fast neutron fluences up to his maximum fluence $\left(10^{25}\right.$ $\mathrm{n} / \mathrm{m}^{2}$ ). Growth of annealed tubing appeared to saturate at a fluence of $4 \times 10^{24} \mathrm{n} / \mathrm{m}^{2}$ and a fractional length change of $4 \times 10^{-4}$. However, subsequent data taken by other investigators has indicated that saturation is not determined by fluence or net growth.

Harbottle $[$ IV-14] has reported the difference in growth strains of transverse and longitudinal strips cut from zircaloy- 2 pressure tubes. The strips were annealed and then irradiated at $-196^{\circ} \mathrm{C}, 40^{\circ} \mathrm{C}$, and $80^{\circ} \mathrm{C}$. Basal pole texture was found to be $13 \%$ in the direction of the tube axis and $36 \%$ in the circumferential direction both before and after the cutting and annealing process. Hartbottle's differential growth strains were converted to approximate values of axial growth strains by using the equation

$$
\frac{1-3 f_{z}}{1-3 f_{\theta}}=\frac{\text { growth strain in axial direction }}{\text { growth strain in transverse direction }}
$$

which is suggested in Reference IV-15.

A somewhat different approach was taken by Daniel [IV-15\& IV-20] in a series of experiments which measured both diameter and length changes of fuel rods. The effects of fuel-cladding interactions and pressure differentials across the cladding on measured changes in rod length could be separated from the effect of cladding growth since no fuel-cladding mechanical interaction was present in one experiment series. The separation was achieved by noting that the expected ratio of length to diameter changes is very different for fuel-cladding interactions, creep due to pressure differentials across the rod, and irradiation-induced growth. In particular, the change in diameter due to growth was predicted to be very small for typical cladding diameters and textures. The measured change in diameter could thus be used to determine the change in length due to growth by simply extrapolating to zero change in diameter with data which did not contain fuel-cladding mechanical interactions.

Daniel determined the fractional change in length due to irradiation growth at two values of fluence. His results ${ }^{[a]}$ are particularly significant because they provide a measure of the growth of annealed cladding at high fluence and they do not show the saturation which Kreyns $\lfloor|V-| Y\rfloor$ measured.

4,1,4 Analysis of Irradiation-Induced Growth Factors. The effects on irradiation growth of texture, fast neutron fluence, irradiation temperature, and cold work are analyzed in the following paragraphs. The fast neutron flux ${ }^{[I V-14]}$ (in addition to fluence) and the residual stress $[[\mathrm{I}-21]$ in the tubing may affect growth but no attempt has been made to

[a] A growth component of strain equal to $7.5 \times 10^{-4}$ at a fluence of $17 \times 10^{24}$ and a growth strain of $2.7 \times 10^{-4}$ at a fluence $3.1 \times 10^{24} \mathrm{n} / \mathrm{m}^{2}$ were indicated by Daniel [IV-15, IV-20] 
include these effects due to lack of data: Also, no significant difference in the growth rates of zirconium, zircaloy-2, and zircaloy 4 has been reported, so no distinction between their growth rates has been incorporated into the model.

4.1.4.1 The Effect of Texture on Irradiation-Induced Growth. Modeling of texture effects has been carried out under the assumption that irradiation-induced growth is caused by the migration of vacancies and interstices created by irradiation but only the gross external features of growth are modeled. That is, the migration is modeled by picturing the net effect of irradiation as the removal of entire basal planes of atoms from individual grains and the relocation of atoms to form new planes perpendicular to the basal planes. This picture and the techniques outlined in Reference IV-15 lead to the conclusion that the effect of texture on axial growth will be approximately proportional to $\left(1-3 f_{z}\right)$ where $f_{z}$ is the effective fraction of cells with their $c$ axis parallel to the tubing axis as determined by X-ray diffraction studies. In Figure IV-23 the data of Figure IV-22 is replotted with texture normalized to $\mathrm{f}_{\mathrm{z}}=0.05$ by multiplying Harbottle's results [V-14] (having $f_{z}=0.13$ ) by $[1-3(0.05)] /[1-30(9.13)]$. A typical value of $f_{z}=0.05^{[I V-15]}$ has been assumed for the measurements by Kreyns on tubing at $300^{\circ} \mathrm{C}$.

4.1.4.2 The Effect of Fast Neutron Fluence on Irradiation-Induced Growth. Many investigators have treated the effect of fast fluence by fitting $q$ in the empirical expression

$$
\text { growth strain } \propto(\text { fluence })^{q}
$$

to the data ${ }^{[I V-14,}$ IV-22]: with resultant values of $q$ in the range $0.3-0.8$. Although good agreement can be obtained by allowing the value of $q$ to vary for each set of data, the results of such empirical fits are somewhat misleading. Hesketh ${ }^{[\mathrm{IV}-22]}$ has derived a dependence on the square root of fluence ( $q=0.05$ in Equation IV-29) and data from individual irradiations have not demonstrated a clear departure (other than saturation effects) from this rule. This point is illustrated in Figure IV-24 by showing a plot of axial growth as a function of the square root of the fluence.

Departures from an exponent of $q=0.5$ would be indicated by curvature of the data in Figure IV-24. Except for apparent saturation effects on annealed tubes at $300^{\circ} \mathrm{C}$, these departures are much less pronounced than differences due to different temperatures, fluences, and cold work amounts. Moreover, one would expect temperature and flux to modify the effect of a given fluence. Therefore, the exponent in Equation IV-29 is fixed in the model at 0.5 due primarily to the evidence of Figure IV-2.

4.1.4.3 The Effect of Temperature on Irradiation-Induced Growth. The effect of irradiation temperature on axial growth of zircaloy tubes is also illustrated in Figure IV-24. The decrease in slope with increasing temperature is consistent with the suggestion by Harbottle $[\mathrm{IV}-14]$ that growth is proportional to $\phi \exp \left[\mathrm{E}_{\mathrm{m}} / \mathrm{RT}\right]$ where $E_{m}$ is the interstitial migration energy, $\phi$ is the fast neutron flux, and $R$ is the gas constant. The current model approximates the temperature dependence with

$$
\frac{\Delta \mathrm{L}}{\mathrm{L}} \propto \exp \left[\mathrm{T}_{\mathrm{o}} / \mathrm{T}\right]
$$




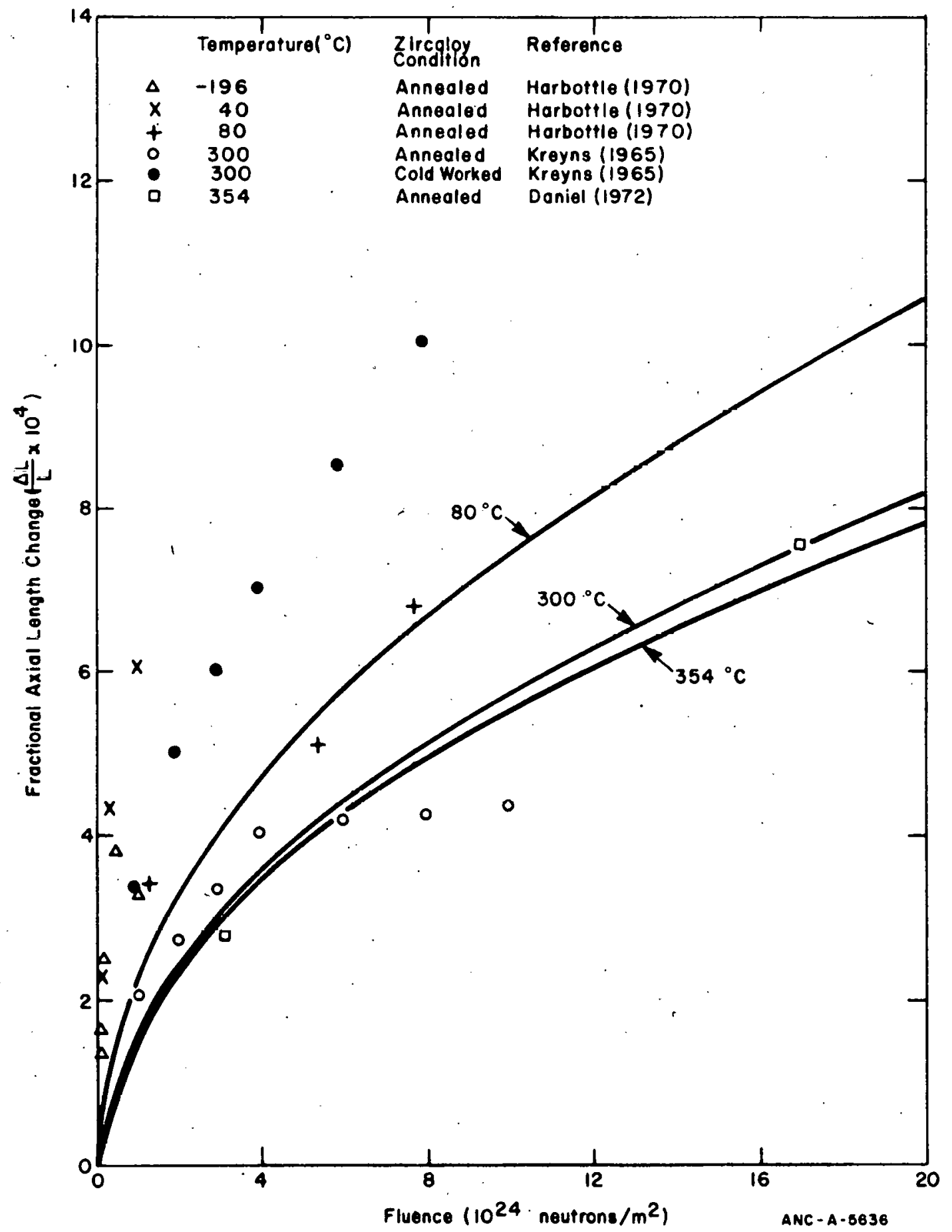

Fig. IV-23 Model predictions and measured values of growth of zircaloy tubes adjusted to a common texture coefficient of $\mathrm{f}_{\mathrm{z}}=0.05$.

where $T_{o}$ is a constant. $E_{m}$ is treated as a constant because there is insufficient data with which to test the complete model or to evaluate $E_{m}(T)$. The empirical value of $T_{0}$ used in the model is $240.8^{\circ} \mathrm{K}$, and was found by fitting Equation IV-23 to the curves in Figure IV-24. 


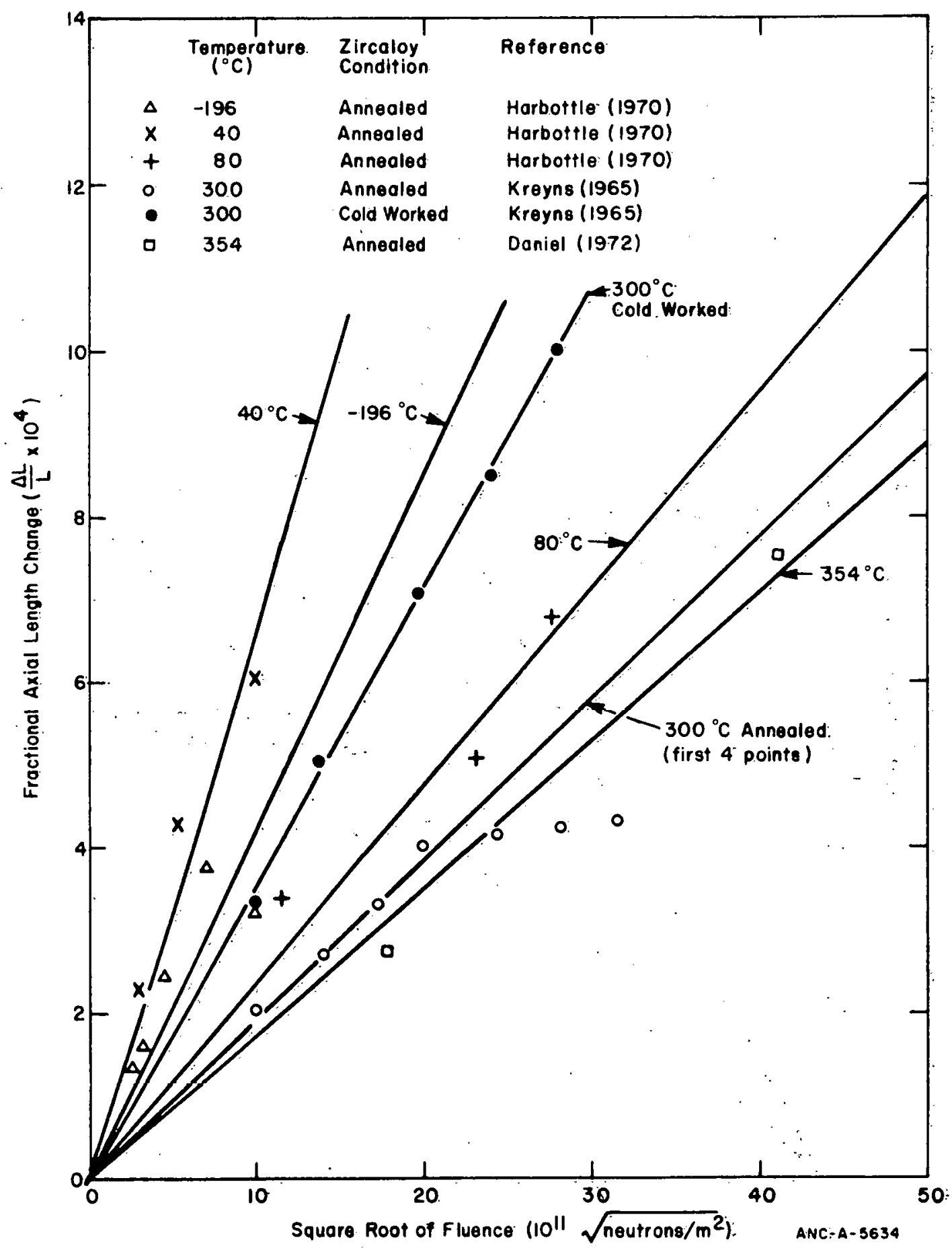

Fig. IV-24 Zircaloy growth versus square root of fast neutron fluence for data adjusted to a common. tube: texture coefficient of $\mathrm{f}_{\mathrm{z}}=0.05$ with linear least squares fits superimposed.

4.1.4.4 The Effect of Cold Work on Irradiation-Induced Growth: The observed effects of cold work have not been successfully explained in detail in the literature. For the present model, general conclusions have been drawn from the available measurements and an emprical expression has been formed. The data taken by. Kreyns ${ }^{[\mathrm{IV}-19]}$ on cold worked zircaloy 4 tubes at $300^{\circ} \mathrm{C}$ agrees very. well with a square root of fluence dependency as shown in Figure IV-25. In order to compare these results 


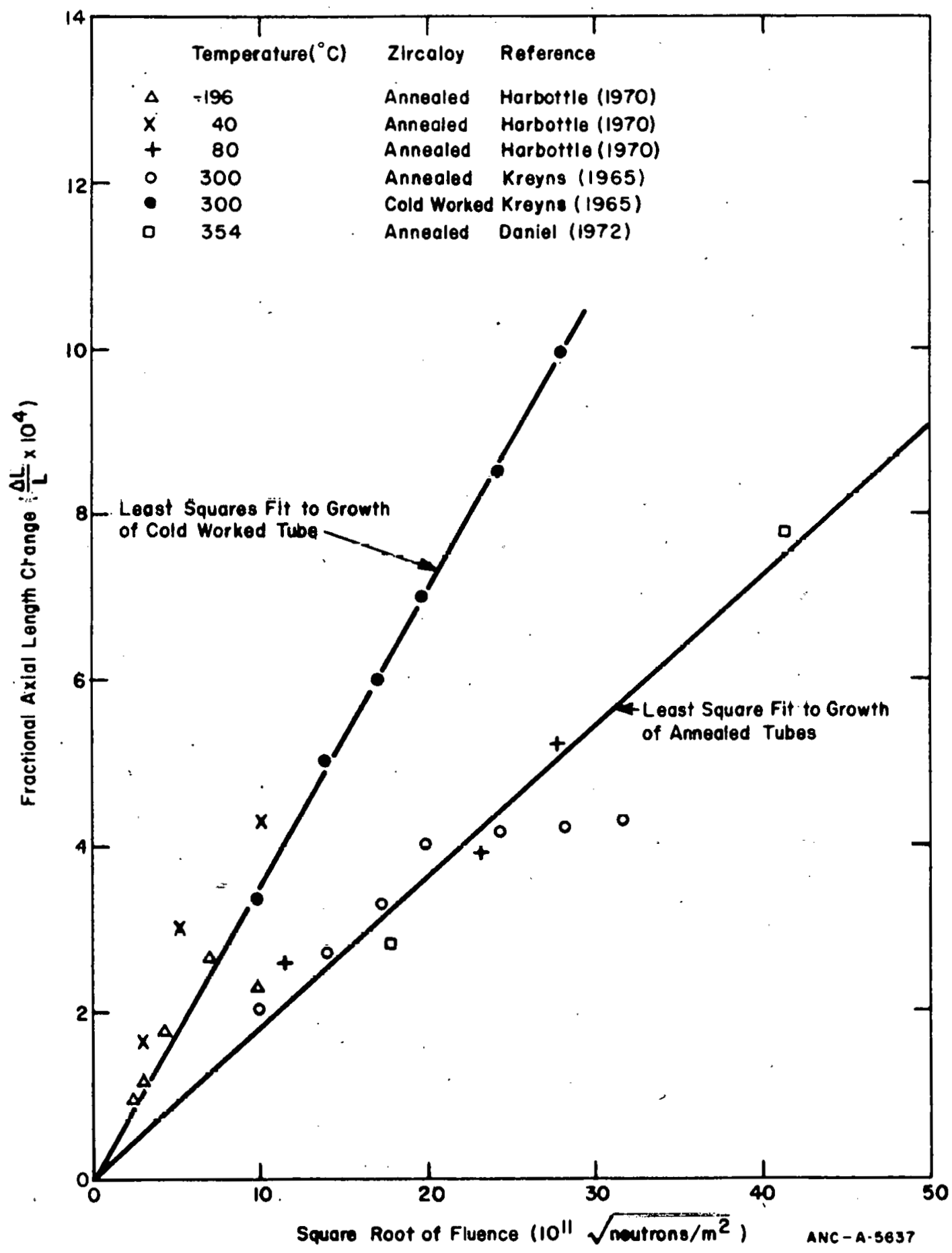

Fig. IV-25 Zircaloy growth versus square root of fast neutron fluence for data adjusted to a common tube texture coefficient of $\mathrm{f}_{z}=0.05$ and to a common temperature of $300^{\circ} \mathrm{C}$, with linear least squares fits superimposed.

with those for annealed tubes, the annealed data shown in Figure IV-24 was normalized to $300^{\circ} \mathrm{C}$ by use of Equation IV-23. Figure IV-25 then indicates that the net effect of cold work is to increase the growth rate in the unsaturated range of fluence. Neither the dependence on square root of fluence nor the intercept at zero fluence is changed by cold working the tubing. 
The only available data on the effect of varying the amount of cold work are reported in Reference IV-23 which indicates approximate irradiation growth fractions in the longitudinal direction of zircaloy -4 plate specimens at $300^{\circ} \mathrm{C}$ as shown in. Table IV-III.

TABLE IV-III

ZIRCALOY GROWTH DATA AS A FUNCTION OF COLD WORK AND FLUENCE

\begin{tabular}{llll}
\hline $\begin{array}{l}\text { Fast } \\
\text { Fluence } \\
\left(10^{24} \mathrm{n} / \mathrm{m}^{2}\right)\end{array}$ & \multicolumn{1}{c}{ Cold Work } \\
\cline { 2 - 4 } 14 & $7.4 \times 10^{-4}$ & $\frac{20 \%}{7.8 \times 10^{-4}}$ & $\frac{78 \%}{17.4 \times 10^{-4}}$ \\
\cline { 2 - 4 } 20 & $8.2 \times 10^{-4}$ & $11.7 \times 10^{-4}$ & $24.4 \times 10^{-4}$ \\
30 & $9.2 \times 10^{-4}$ & $17.3 \times 10^{-4}$ & $36.3 \times 10^{-4}$ \\
\hline
\end{tabular}

The data are reasonably consistent with a linear relationship between growth and the amount of cold work and have been incorporated into the model by assuming a factor of the form $(1+D \times$ cold work). Values of the constant $D$ determined from the data at the three different fluences are listed in Table IV-IV, where

$$
D=\frac{1}{\text { cold work }} \quad\left[\frac{\text { Growth with cold work }}{\text { Growth without cold work }}-1\right]
$$

TABLE IV-IV

DETERMINATION OF COLD WORK COEFFICIENT

\begin{tabular}{lc}
\hline $\begin{array}{l}\text { Fast } \\
\text { Fluence }\end{array}$ & \\
$\left(10^{24} \mathrm{n} / \mathrm{m}^{2}\right)$ & $\mathrm{D}$ \\
\hline 14 & 1.7 \\
20 & 2.0 \\
30 & 3.8 \\
\hline
\end{tabular}

The value $D=2.0$ given by the data at the lower fluences is used in the model since the measured values of growth with $0 \%$ cold work (Table IV-III) show gross saturation effects similar to the effects apparent in the high fluence data of Kreyns [iV-19]. The model thus sacrifices a description of these gross saturation effects in order to fit the cold worked data and the majority of the annealed tubing data.

4.1.5 Evaluation of the Model and its Uncertainty. The normalization of all the annealed data to identical conditions (texture coefficient $f_{z}=0.05$, temperature $=300^{\circ} \mathrm{C}$ ) as shown in Figure IV-25 provides a test of the model. The model predicts irradiation 
growth reasonably well except for data taken at fluences less than $10^{24}$ neutrons $/ \mathrm{m}^{2}$ and except for greater than normal saturation effects seen in some annealed samples. Figure IV-22 gives the same conclusion and also indicates the relative effect of the temperature, texture, and fluence variables as predicted by the model. (The factor A uséd in Equation IV-27 for these curves was derived from a linear least squares fit to the data of Figure IV-25.)

Further refinement of the model to explain the relatively high values measured at low fluence and to explain the gross saturation effects observed on some samples has not been attempted. In the low fluence case there are competing processes that may explain the high values sometimes found, and there is no way to distinguish between them without additional data. These effects are:

(1) Stress relief calssing additional length shanges [IV-21]

(2) Variation in fast flux causing different growth rates [IV-14]

(3) Variation in interstitial migration energy with temperature causing error in the temperature model.

Similar problems exist with attempts to model the gross saturation effects observed in some experiments by Kreyns [IV-19] using tubing and by Fidleris [IV-23] using plate samples. There are sufficient data to indicate clearly that these saturation effects in growth are not simply a function of the fluence or the growth strain. However, little data are available to consider correlations between saturation and other parameters.

An estimate of the uncertainty can be obtained by comparing predictions of the model with data which were not used in formulating the model. For example, the plate specimen data listed in Table IV-III for $0 \%$ cold work (and $300^{\circ} \mathrm{C}$ ) was not used to formulate the predicted growth of annealed tubes. When this data is compared with the model predictions for annealed growth at $300^{\circ} \mathrm{C}$, a discrepancy of approximately $10 \%$ is found. This $10 \%$ discrepancy is consistent with the scatter of the data at fluences above $10^{24} \mathrm{n} / \mathrm{m}^{2}$ in Figure IV-25 and thus represents a reasonable estimate of the model's uncertainty in the temperature range from 40 to $360^{\circ} \mathrm{C}$.

The uncertainty for temperatures outside of this range and for fluences less than $10^{24}$ $\mathrm{n} / \mathrm{m}^{2}$ may be substantially greater than $10 \%$. In the low fluence range, inspection of Figure IV-25 suggests uncertainties on the order of $100 \%$. Such large discrepancies may be due to stress-relief effects $\left[\right.$ IV-21]. For temperatures much outside the range 40 to $360^{\circ} \mathrm{C}$, increased error will be caused by the presence of different modes of atomic interstitial or vacancy migration causing different rates of zircaloy growth. 


\subsection{Summary of Haldèn Recycle Plutonium Experiment \\ P. E. MacDonald}

Analysis of fuel behavior during an accident depends critically upon knowledge of fuel rod conditions during normal (steady state) operation. Especially important are the temperature distributions of the fuel pellets, the geometry of fuel rod components, the internal gas pressure and composition, the amount of restructuring and cracking of the -pellets, and the mechanical strains and irradiation damage in the cladding. All of these parameters are affected by the rod design variables at beginning-of-life and; subsequently, by the degree of burnup and the power history.

Presented below is a summary of the data which were obtained during the steady state irradiation of the instrumented fuel assembly (IFA) 226 in the Halden Heavy Boiling Water Reactor (HBWR) located in Halden, Norway. Fuel rod elongation (axial deformation), fuel centerline temperature, and fuel rod internal pressure data are presented as a function of power and time for rods with various initial pellet-to-cladding gap sizes and fuel densities. These data will facilitate development of analytical models describing:

(1) Fuel pellet-cladding mechanical interactions as a function of power, burnup, fuel density, and initial pellet-cladding diametral gap

(2) Fuel density, pellet-cladding gap, and temperature effects on fission gas release and rod internal pressure

(3) Pellet-to-cladding gap, fuel density, and irradiation effects on mixed oxide fuel temperatures

(4) Fuel rod axial strains as a function of power and burnup.

The test rods were designed and fabricated by Nuclear Fuel Services, Incorporated (NFS) of Rockville, Maryland, and are being irradiated in the Halden HBWR by the Aerojet Nuclear Company in conjunction with the NRC participation in the OECD Halden Reactor Project. The fuel is depleted uranium oxide enriched with recycled plutonium generated in the Big Rock Point Reactor. The cladding is zircaloy-4. The rod dimensions are typical of current commercial PWR designs and highly characterized prior to irradiation. The length, density, and diameter of every pellet was measured and recorded. After sorting, rods with various exact combinations of fuel density and pellet-to-cladding gap were constructed. Cladding dimensions were measured with respect to the location of individual pellets. Figure IV-26 lists the nominal dimensions. Figure IV-27 presents measured pellet and cladding: diameter data as a function of length, illustrating the detail in which the rods were characterized. 
- FUEL ROD OD

- CLADDING ID

- CLADDING THICKNESS

- PELLET-CLADDING GAP

- PELLET OD _._. 0.366 in.

- PELLET LENGTH_....... $0.591 \mathrm{in.}$

- PELLET DENSITY _........ $94.5 \%$

- DISH FRACTION

- FUEL STACK LENGTH

- ROD PRESSURE _............ 15 psi

Fig. IV-26 Fuel rod and pellet characteristics.

The assembly contains twelve mixed plutonium-uranium oxide fuel rods approximately 33 inches long arranged in two hexagonal clusters as shown in Figure IV-28. A range of instruments was chosen which allowed for an accurate monitoring of overall assembly performance as well as the dynamic mechanical and thermal behavior of individual fuel elements during irradiation. The rods in IFA-226 are instrumented with five diaphragm type rod internal pressure sensors (Figure IV-29), six miniature differential transformers for cladding elongation measurement (Figure IV-30), and four tungsten/tungsten-rhenium fuel centerline thermocouples (Figure IV-31). A turbine flowmeter and two coolant thermocouples are mounted in the assembly inlet and outlet, respectively. Eight self-powered beta current neutron detectors monitor radial and axial flux distributions.

The readings from all instruments are recorded every 15 minutes. Local power is calculated for each elongation, temperature, or pressure sensor reading using the neutron detector output and suitable calibration constants determined from the thermal and hydraulic instrumentation. In determining the power history of each test rod, variations in pellet dimensions, fuel density, enrichment, and radial and axial flux distributions are also considered.

IF $\Lambda$-226 began irradiation on November 24, 1971, and up to July 1975 reached approximately $32,000 \mathrm{MWD} / \mathrm{MTU}$ assembly average burnup. The rods are cooled by natural circulation of heavy water at 34 atmospheres pressure and approximately $240^{\circ} \mathrm{C}$. Typical values for the thermal and test flux are: 3.0 to $3.6 \times 10^{13} \mathrm{n} / \mathrm{cm}^{2} \mathrm{sec}$ and $5 \times 10^{11} \mathrm{n} / \mathrm{cm}^{2}$ sec respectively.

4.2.1 Fuel Rod Elongation Data. Figure IV-32 shows initial power ramp elongation data as a function of the rod peak linear heat rating. Gap and density are shown to have respective effects on the power level at which interaction begins and the rate of elongation 


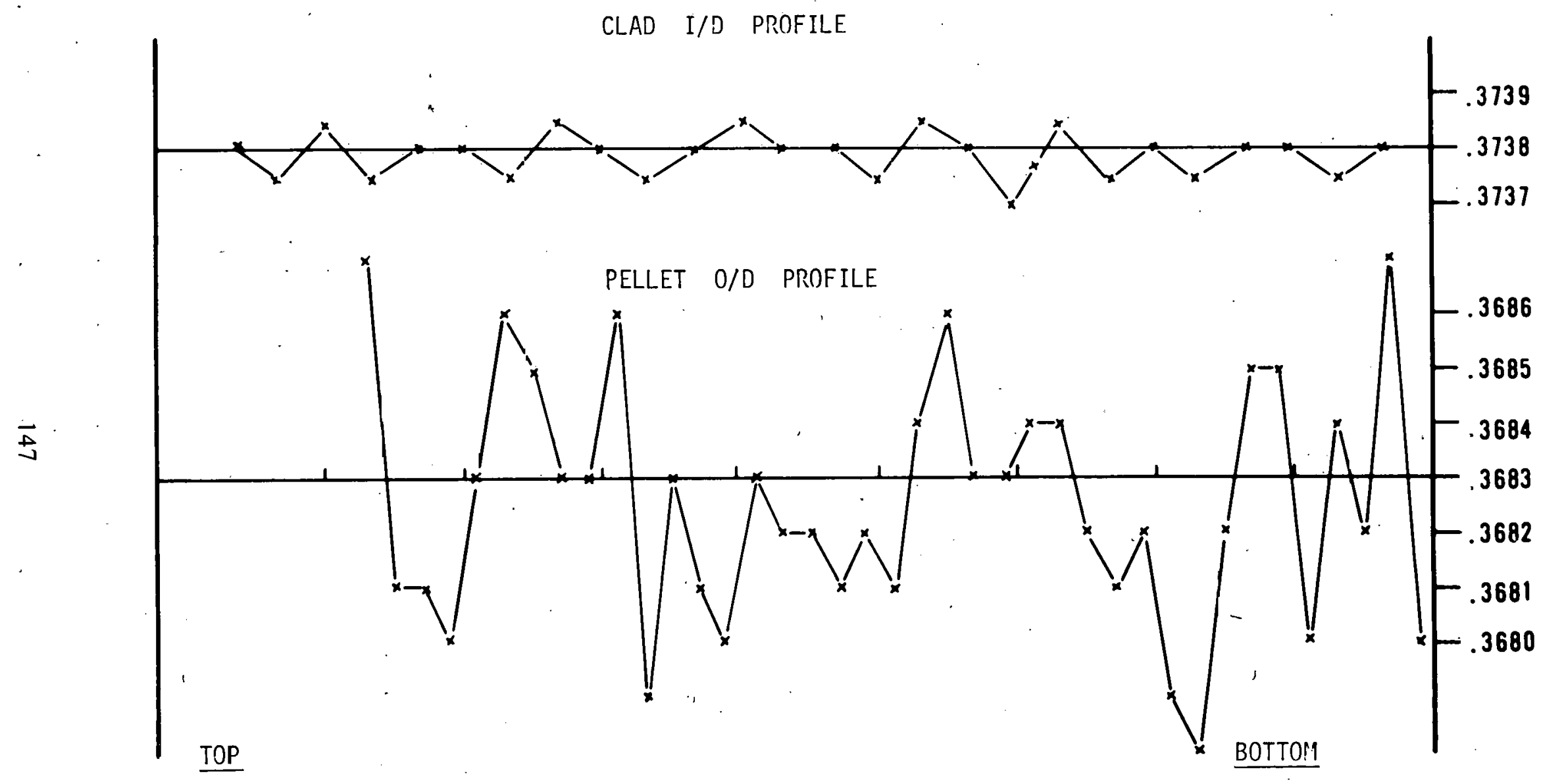

Fig. IV-27 Rod AJ dimensional profile. 


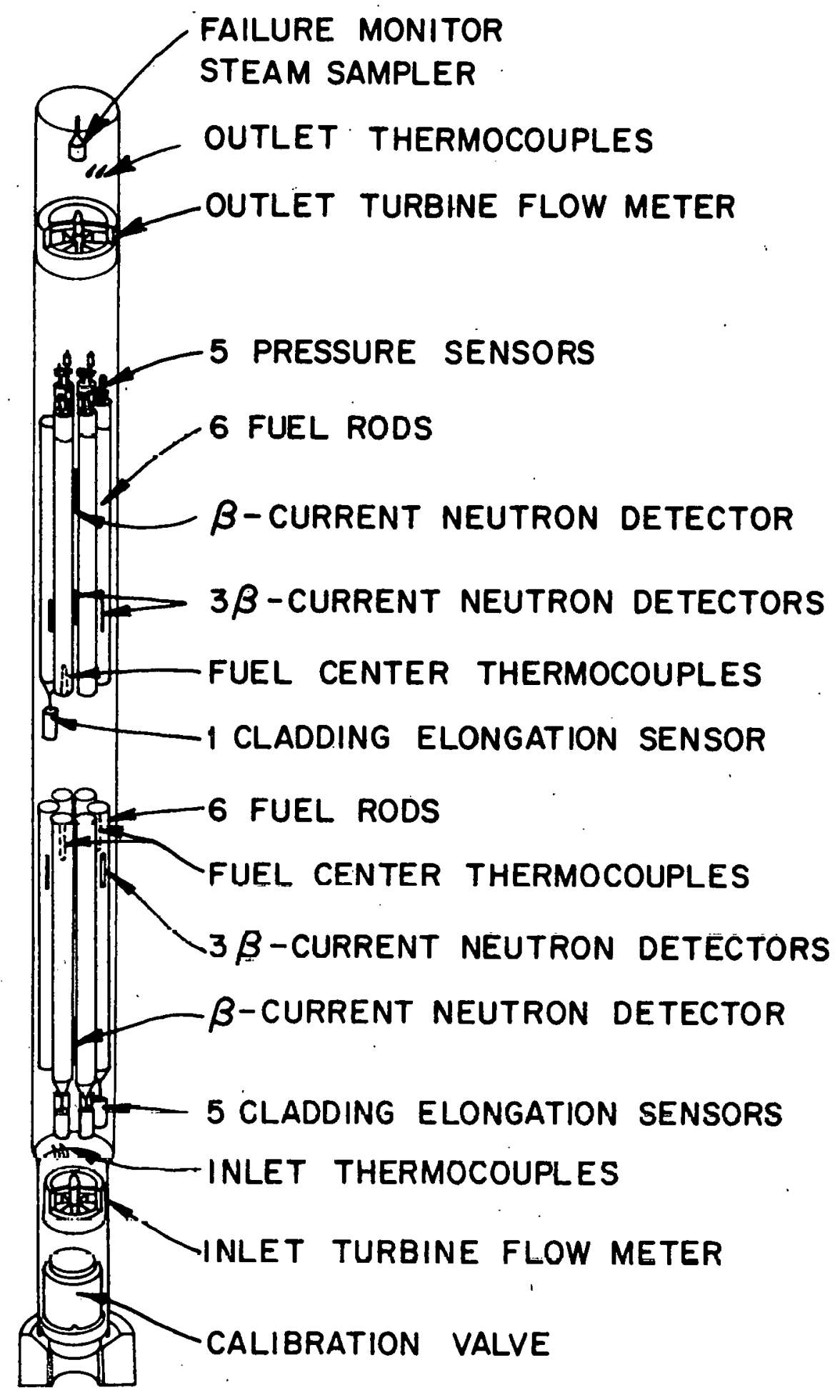

Fig. IV-28 Instrumented fuel assembly-226. 


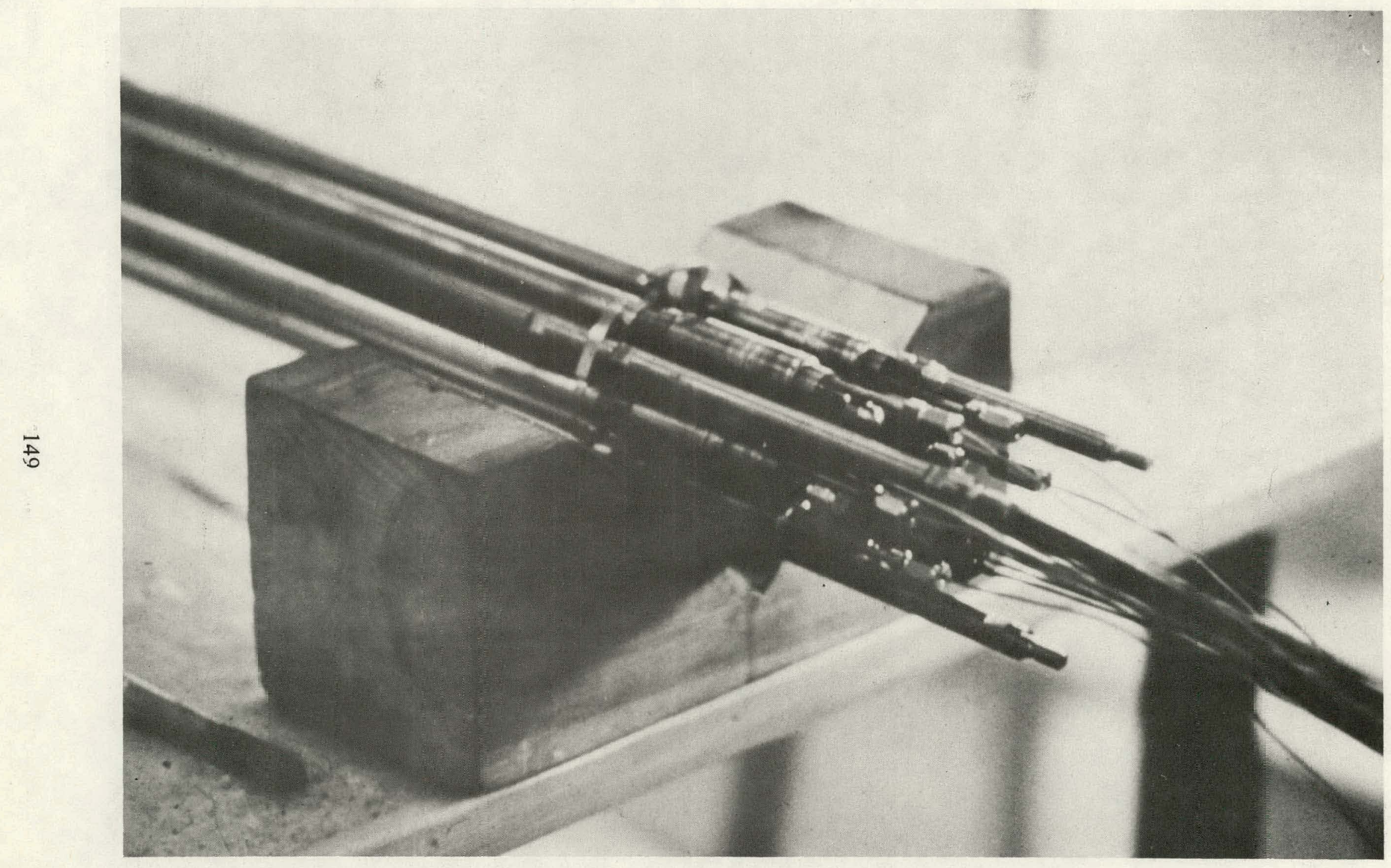

Fig. IV-29 IFA-226 showing pressure sensors. 


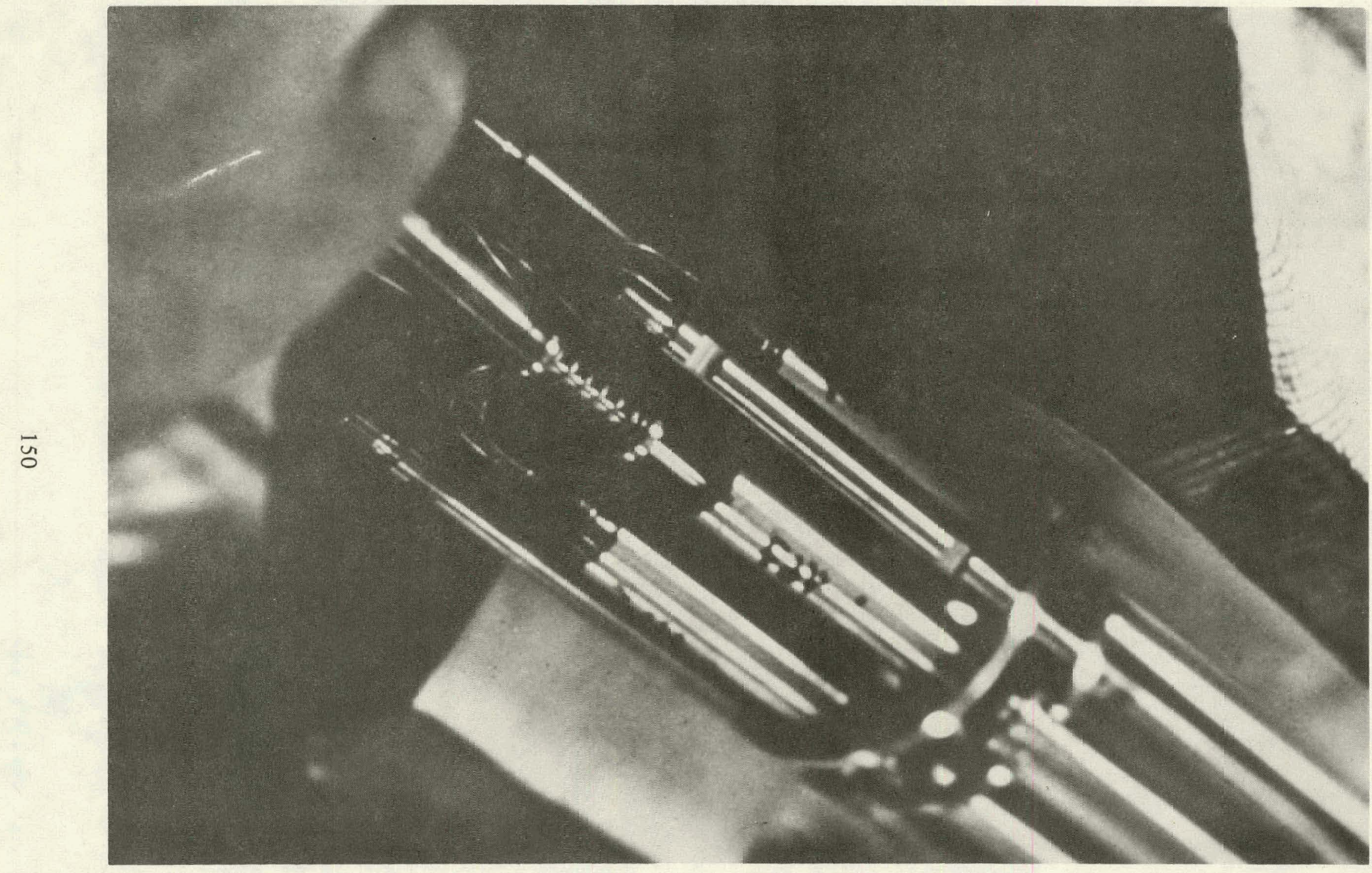

Fig. IV $\approx 0$ IF $\triangle-226$ showing cladding elongation sensors. 


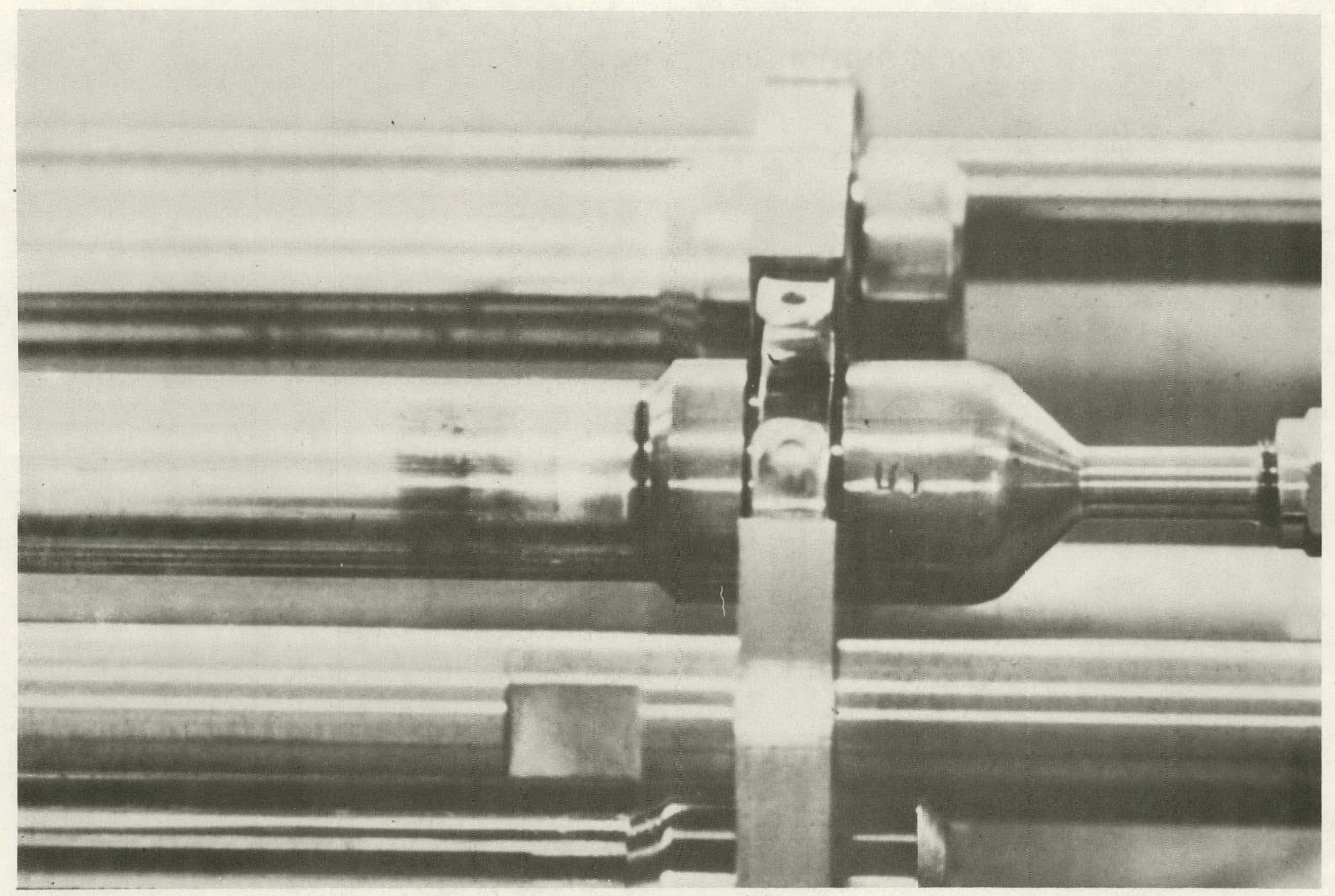

Fig. IV-31 IF A-226 showing centerline thermocouple. 


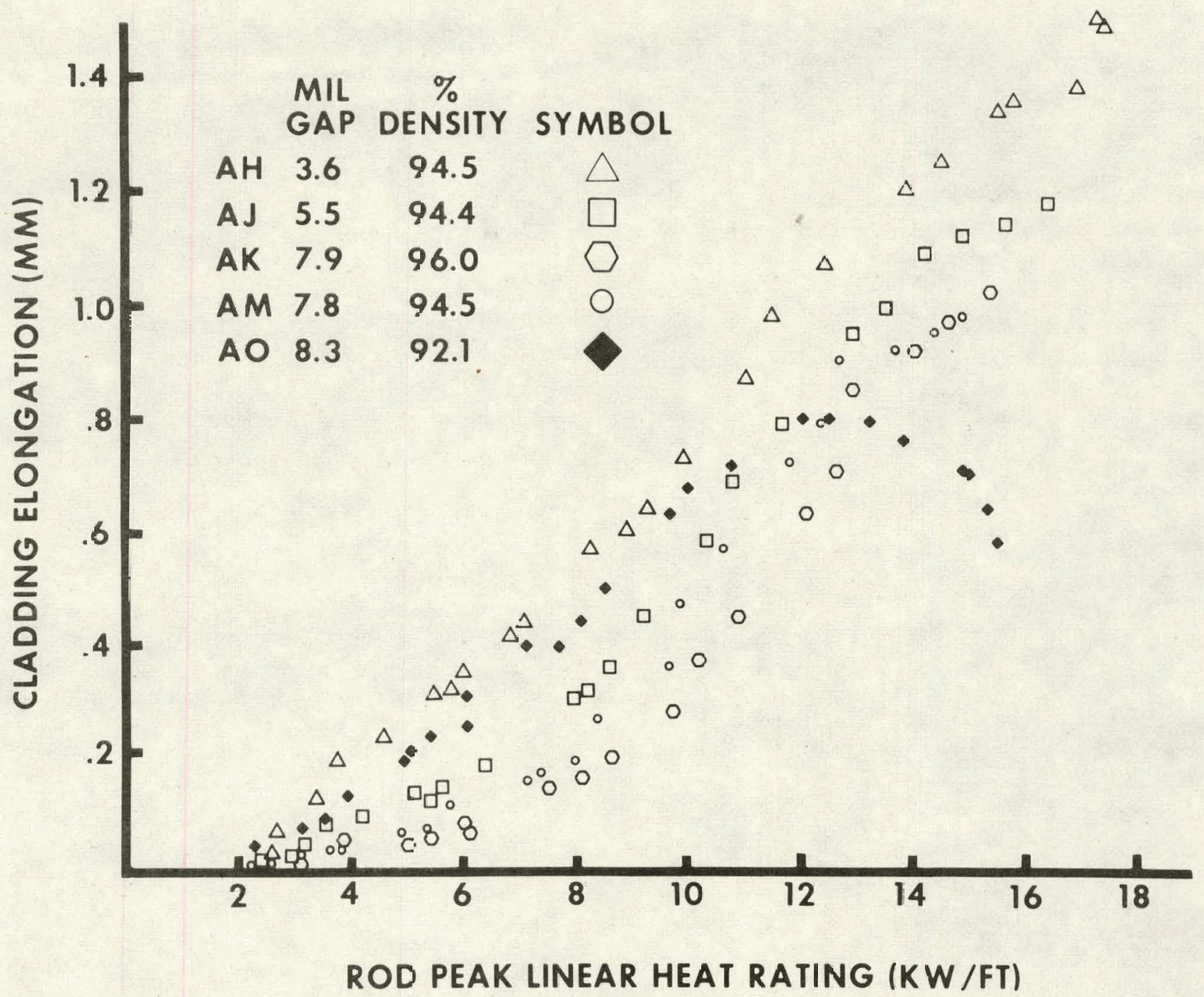

Fig. IV-32 Initial rod elongation vs. rod peak power (zero burnup). 
after this point. In all cases the nominal hot gap is not calculated to have closed in the range of peak powers experienced by the rods, but nonetheless, the existence of mechanical interference is obvious. Apparently, even the low heat ratings at the very beginning of the power ramp induce fuel cracking and non-uniform pellet relocations which combine to minimize the effective gap available for accommodating hot fuel. Then; in the course of power cycling operations, thermally induced fuel stack expansion results in axial: compressive forces on the fuel and tensile forces on the cladding. Stress levels continually. seek equilibrium by some combination of radial and axial deformation of both fuel and cladding (Figure IV-33).

Subsequent power cycling causes additional mechanical interaction but the data suggest more uniform behavior as shown for example in Figure IV-34. Fuel and cladding plasticity at high heat loads, during the preceding power cycles, has acted to relax mechanical interaction stresses and acclimate both materials to spatial restrictions (Figure IV-33). The transition between cladding thermal expansion alone and additional deflections caused by mechanical interaction becomes more defined and occurs at power levels more consistent with the closing of a nominal hot gap.

Figures IV-35 and IV-36 compare the effect of pellet-to-cladding gap size and fuel density on the axial deformation of the IFA-226 test rods. Pellet cocking and jamming and the resulting axial deformations do not seem to be sensitive to gap size but do seem to be quite sensitive to fuel density. Low-density. fuel deforms rather easily and local pellet-cladding locking in the $92 \%$ dense fuel rod appears to have been eliminated after only one power cycle. The resultant cladding elastic and plastic axial strains. were also lowest in the low-density fuel rod.

Figures IV-37 through IV-46 present the rod elongation history from November 24, 1971 (beginning of life) through April 1, 1974. It is apparent that (a) the effects of fuel design variables on fuel-cladding mechanical interaction diminish with burnup; (b) the rods increase in length slightly after most, but not all, power cycles; (c) the total amount of rod ratcheting is not large, and (d) after the first few months of irradiation, strong fuel-cladding mechanical interaction does not occur unless the power is raised above previous peak power levels.

4.2.2 Fuel Rod Thermal Data. Fuel pellets crack extensively upon irradiation due both to thermal stesses induced by power changes and to accumulation of gaseous fission products at grain boundaries at high burnup. Therefore, the thermal properties of an irradiated rod will vary depending on the movement of pellet fragments into the gap and the extent and pattern of the pellet cracks (Figure IV-47).

Figure IV 48 presents the IFA-226 fuel centerline temperature data versus power during the first power ramp (zero to full power in 12 hours). Note that except for a very few points, the response is continuous and almost linear. The data extrapolates to the system temperature at zero power. Compared with one another, rods $\mathrm{AO}$ and $\mathrm{AA}$ temperatures show similar relationships with power (standard PWR rod design: 8 mil: gap; 92.5\% dense fuel). Temperatures for the high-density fuel rod AK are somewhat lower, and temperatures for the large pellet-to-cladding gap rod $\mathrm{AE}$ are somewhat higher than the $\mathrm{AO}$ and.AA temperatures, as expected. 


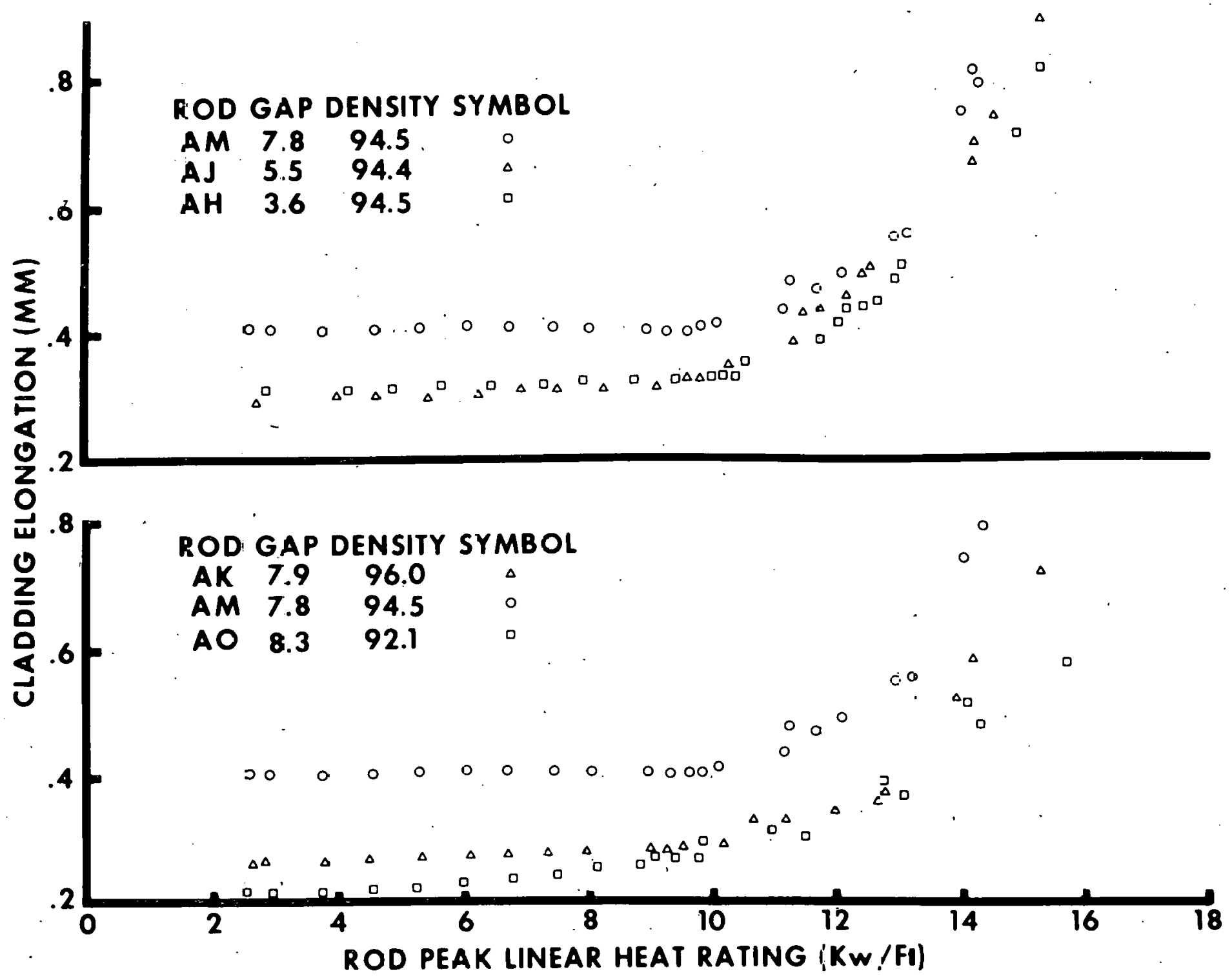

Fig. 1V-33 Rod elongation vs. rod peak power (assembly average burnip 20 MWD/MTM). 


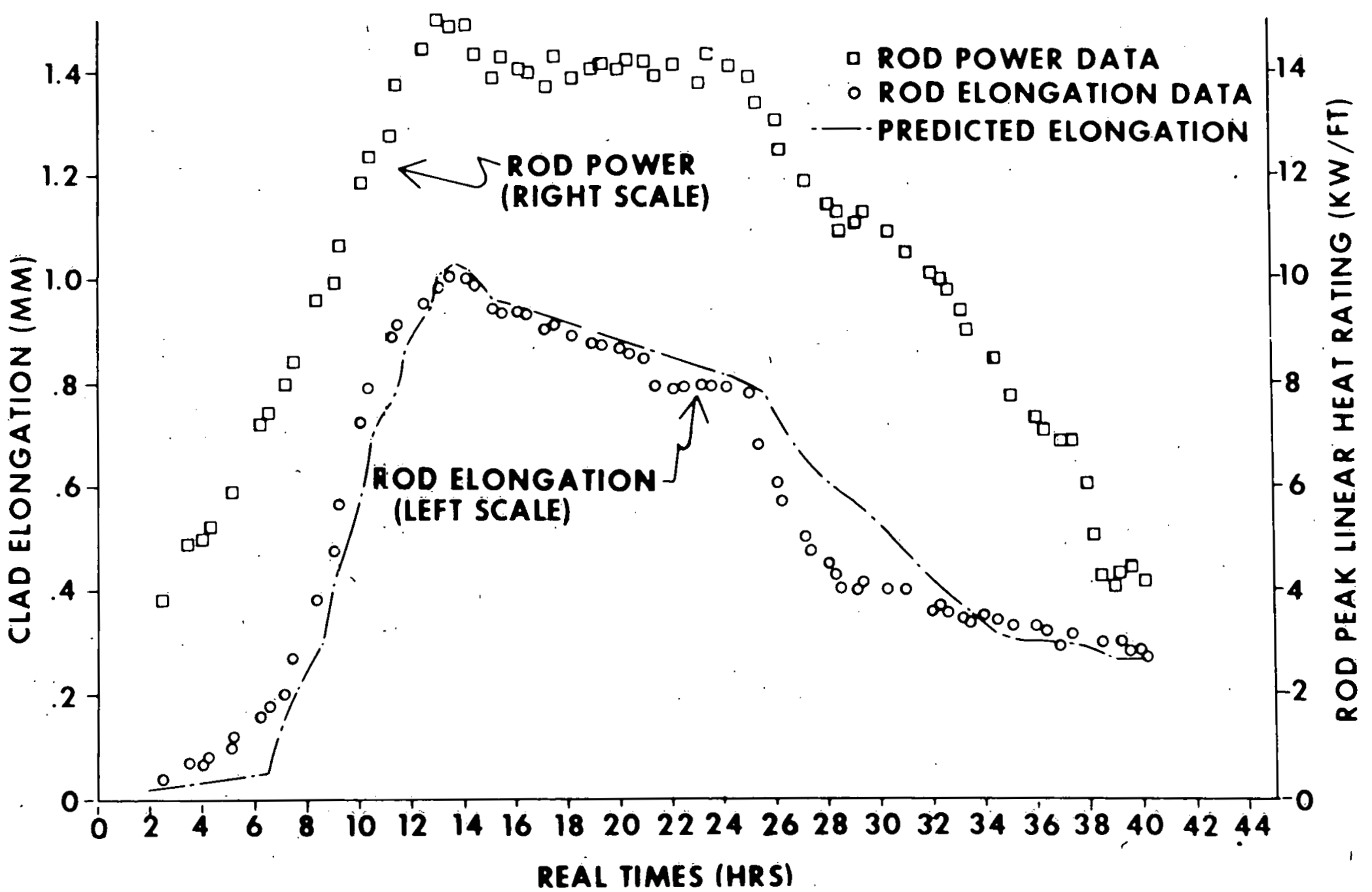

Fig. IV-34 Rod AM elongation vs. time (IFA-226 first power cycle). 


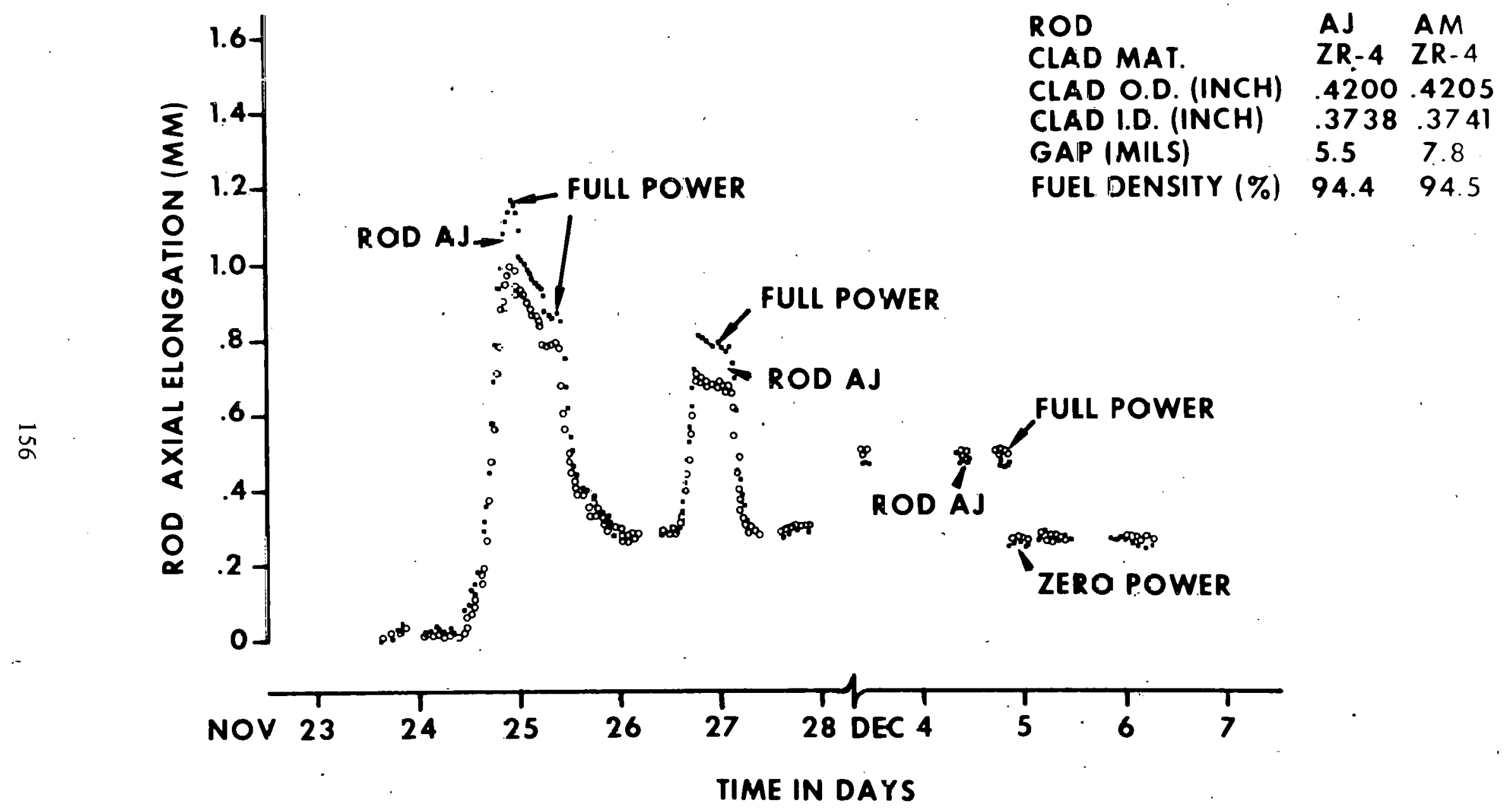

Fig. IV-35 Effect $0:$ pellet-to-cladding gap size on fuel rod axial deforma:ion. 


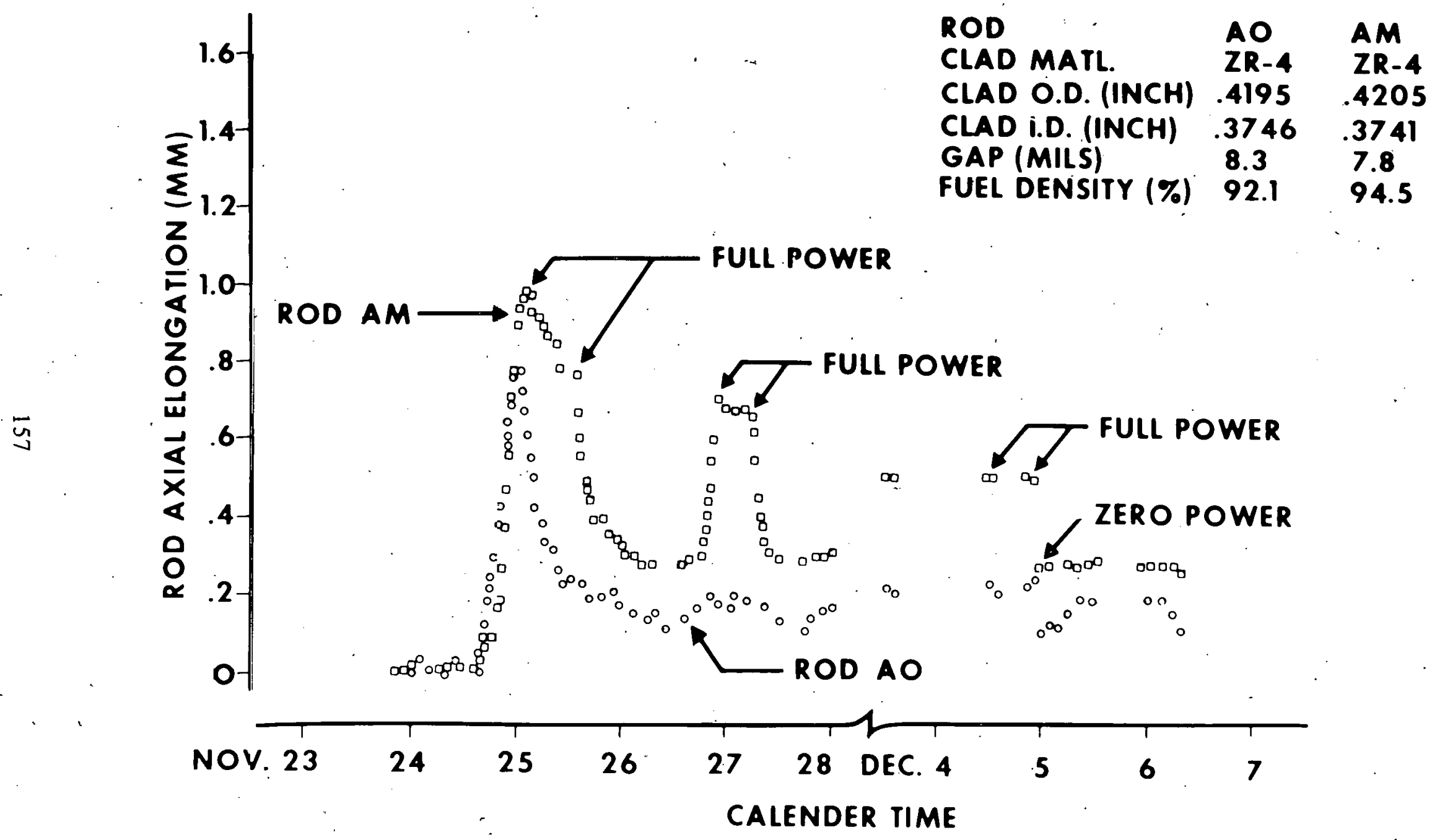

Fig. IV-36 Effect of fuel density on fuel rod axial deformation. 

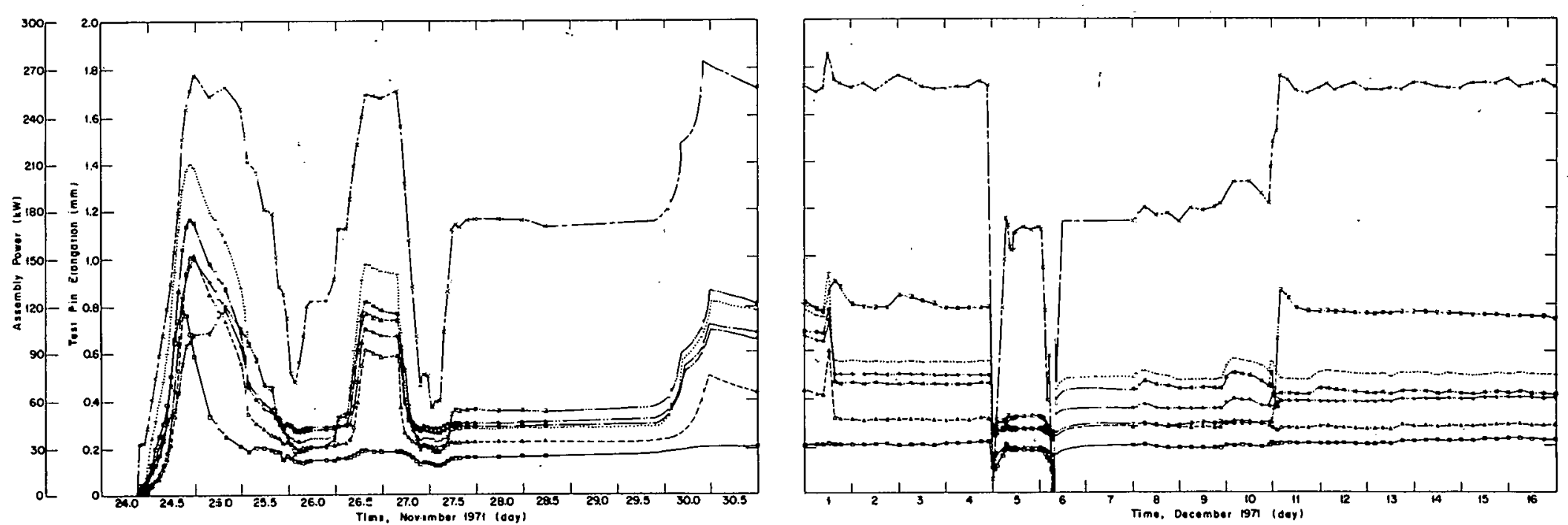

ঢ̄
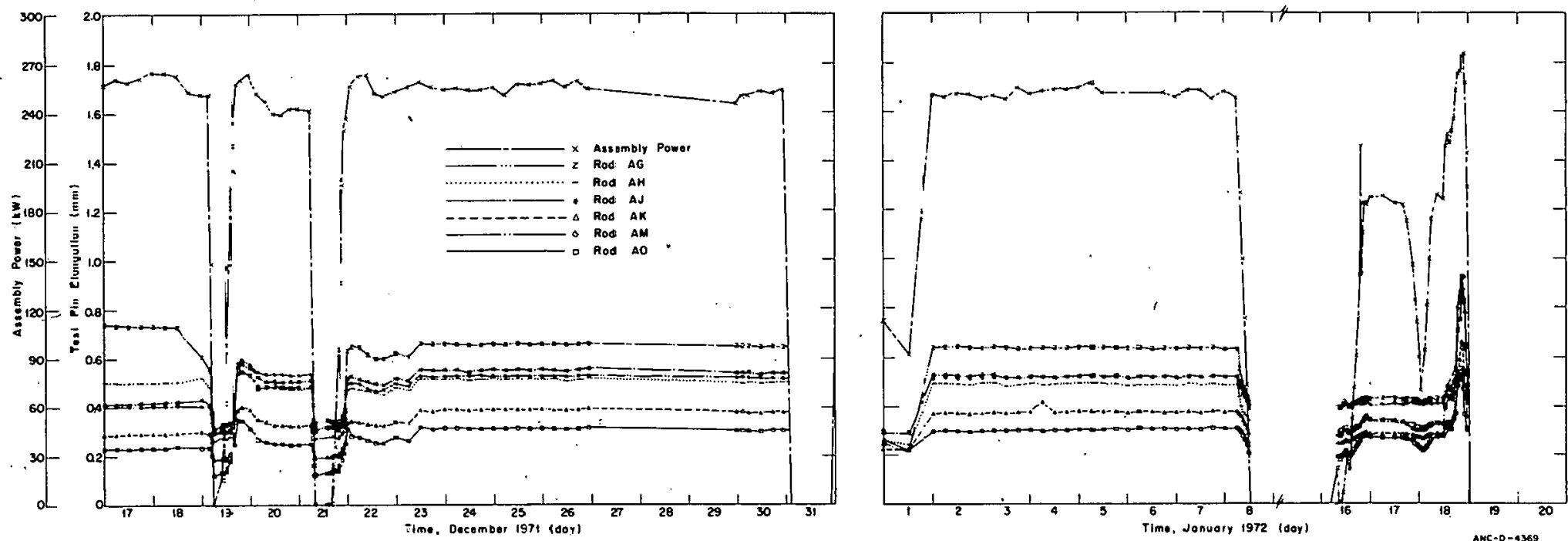

Fig. IV-37 Elongation history of IFA-226 test pins from November 1971 through January 1972. 

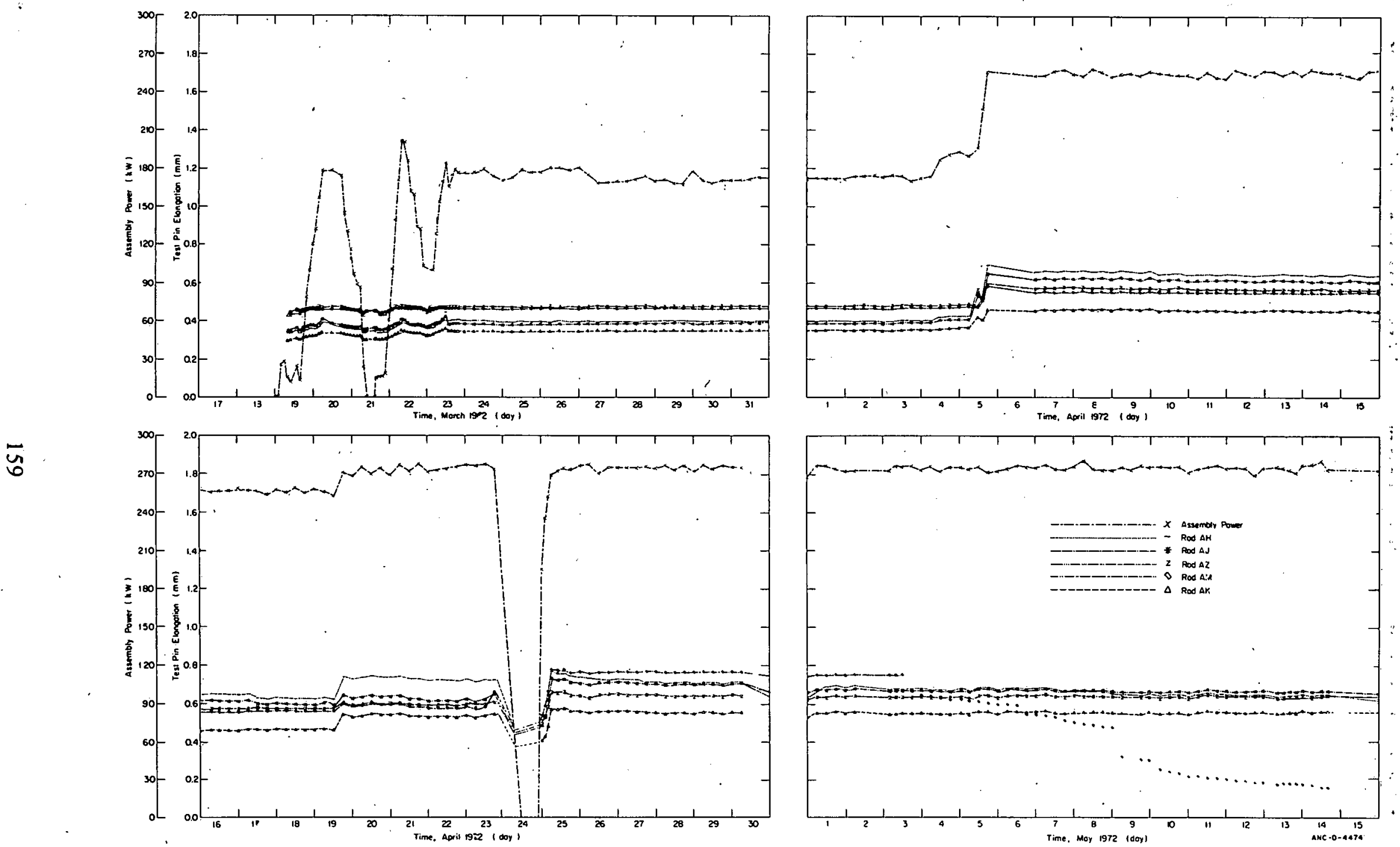

Fig. IV-38 Elongation history of IFA-226 test pins from March 1972 through May 1972. 

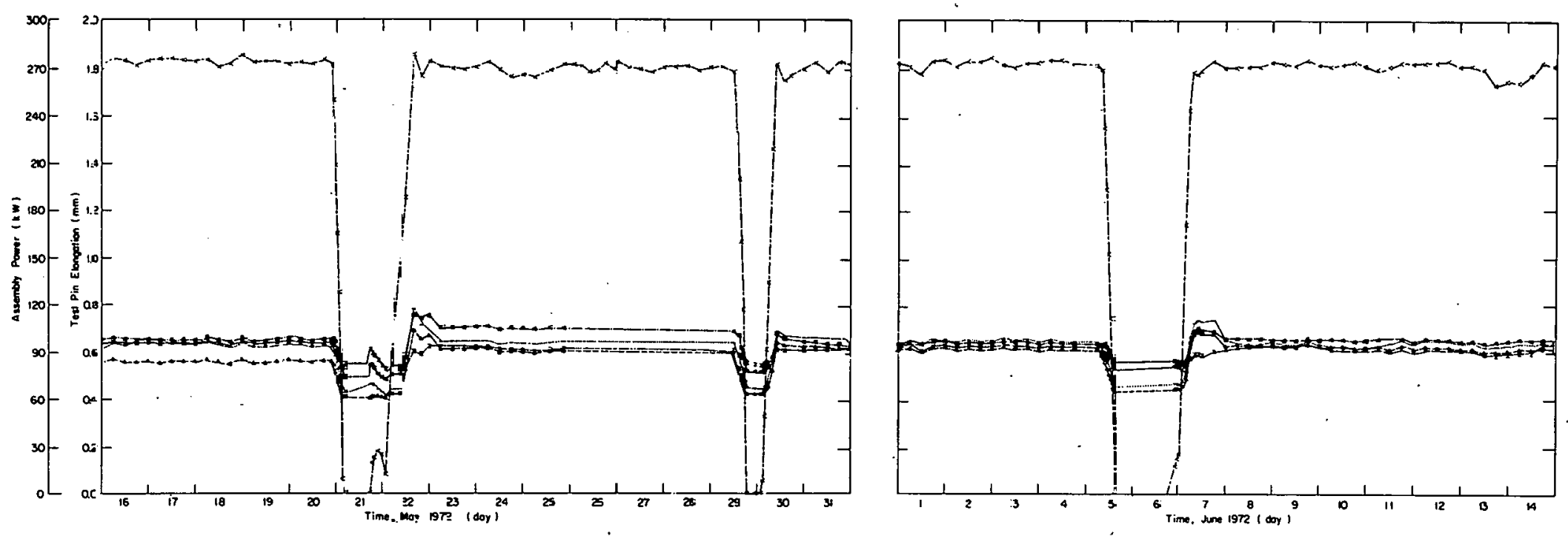

홍
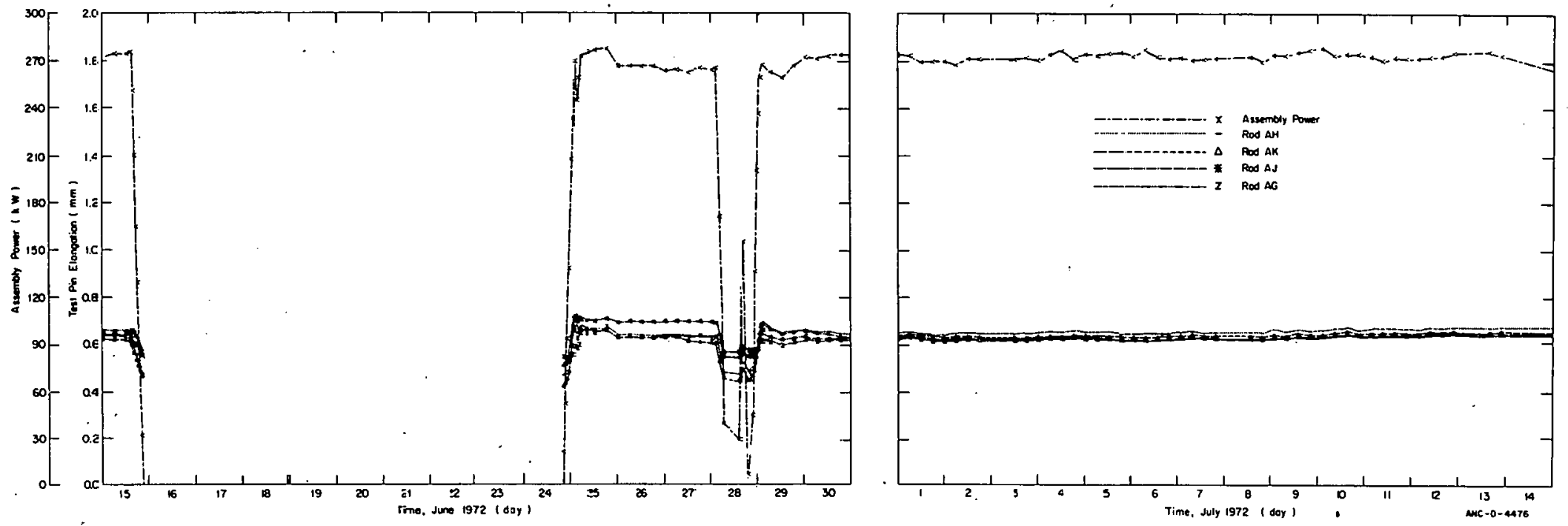

Fig. IV-39 Elonga:ion Kistory of IFA-226 test pins from May 1972 through -uly 1972 

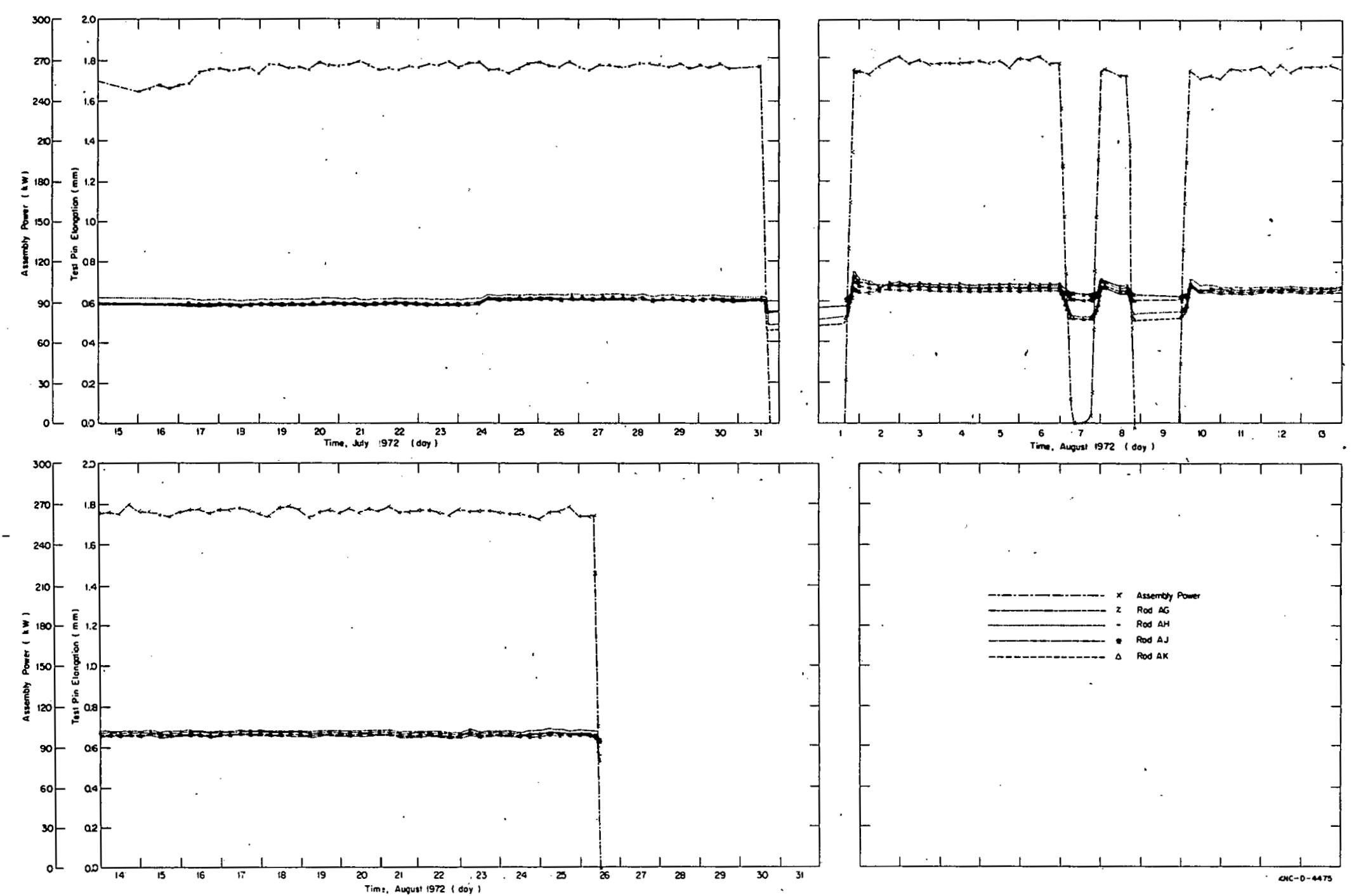

Fig. IV-40 Elongation history of IFA-226 test pins from July 1972 throügh August 1972. 

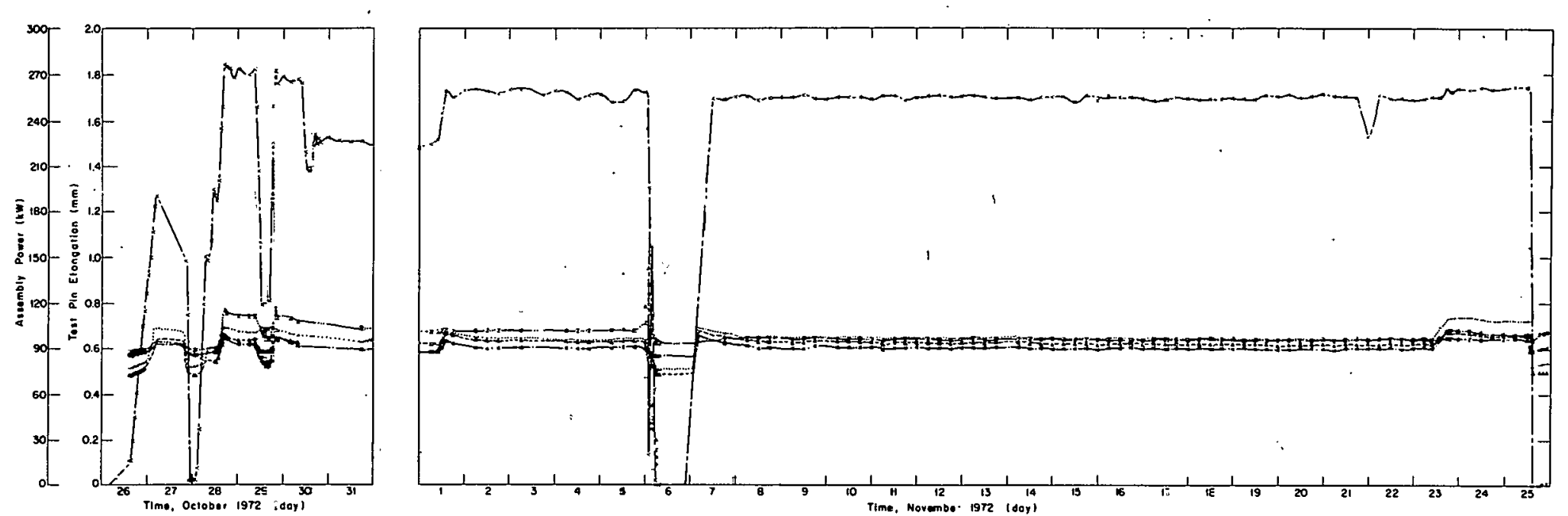

ลำ

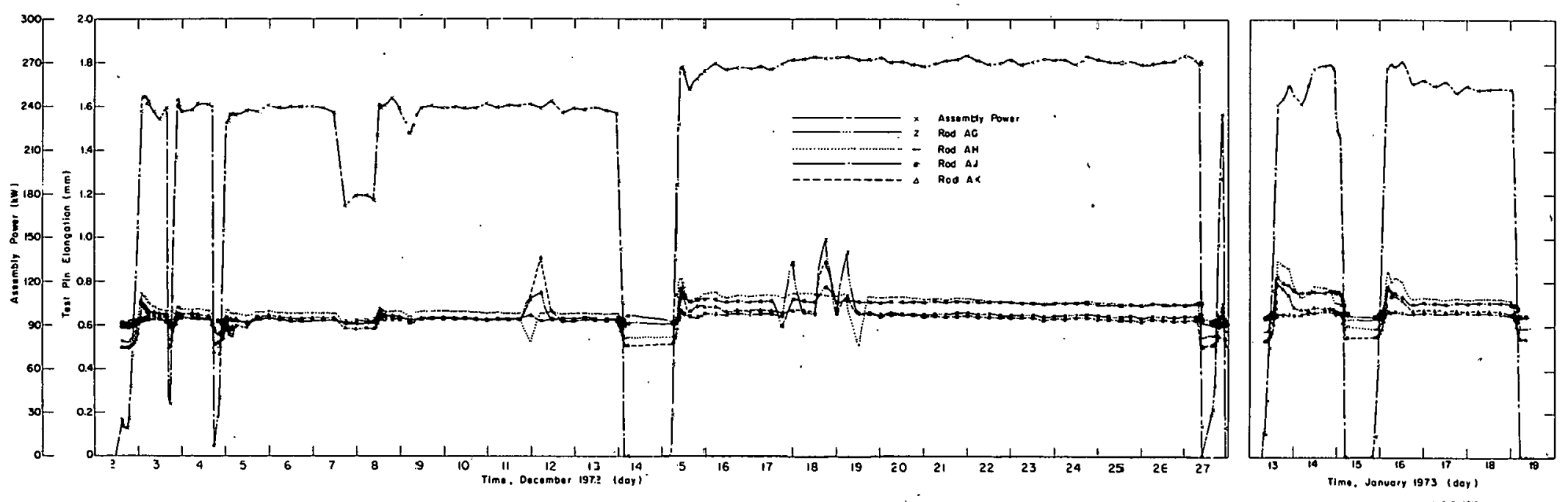

Fig. IV-41 Elongation history of IFA-226 test pins from October 1972 through Jant:ary 1973. 

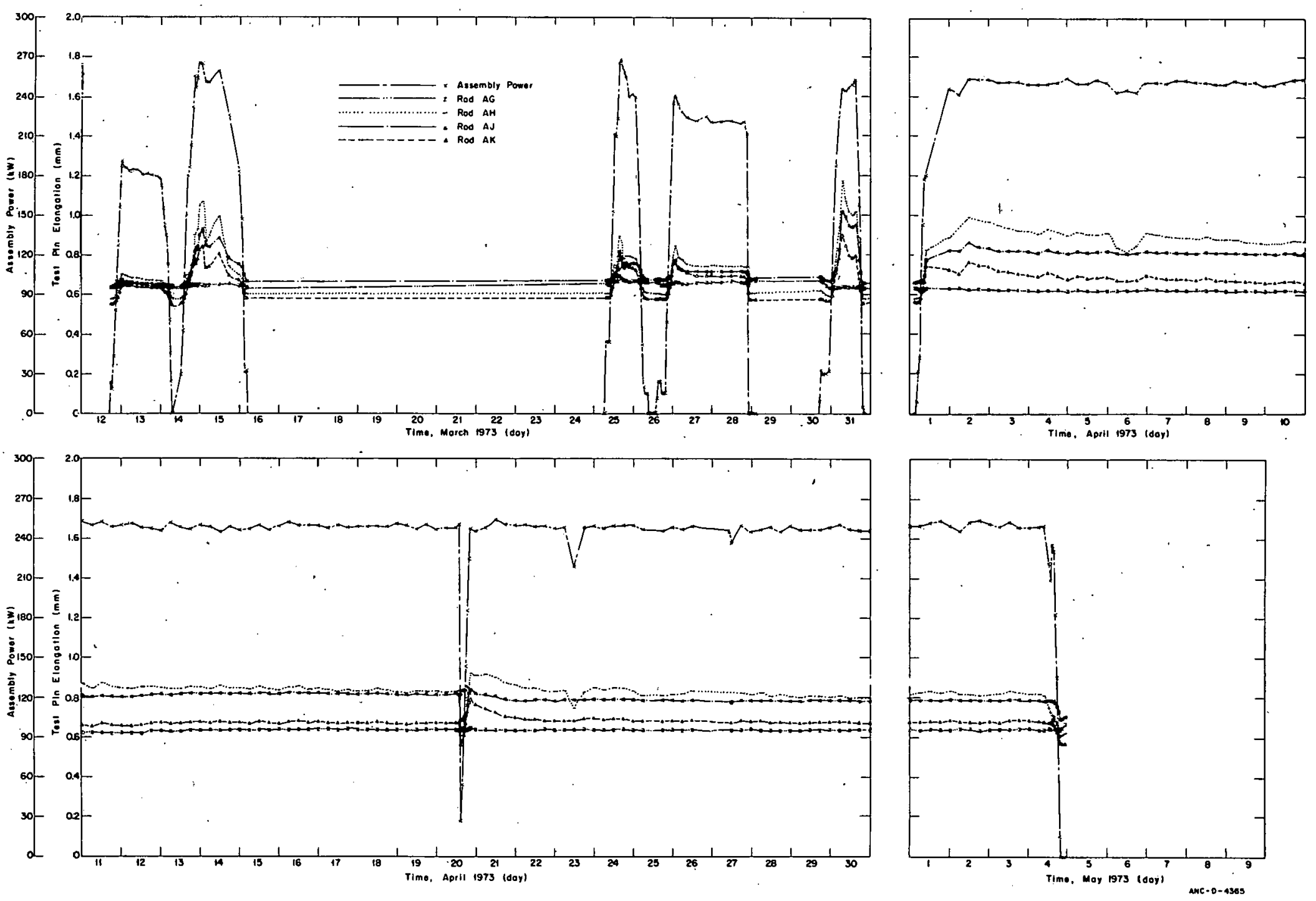

Fig..IV-42 Elongation history of IFA-226 test pins from March 1973 through May 1973. 

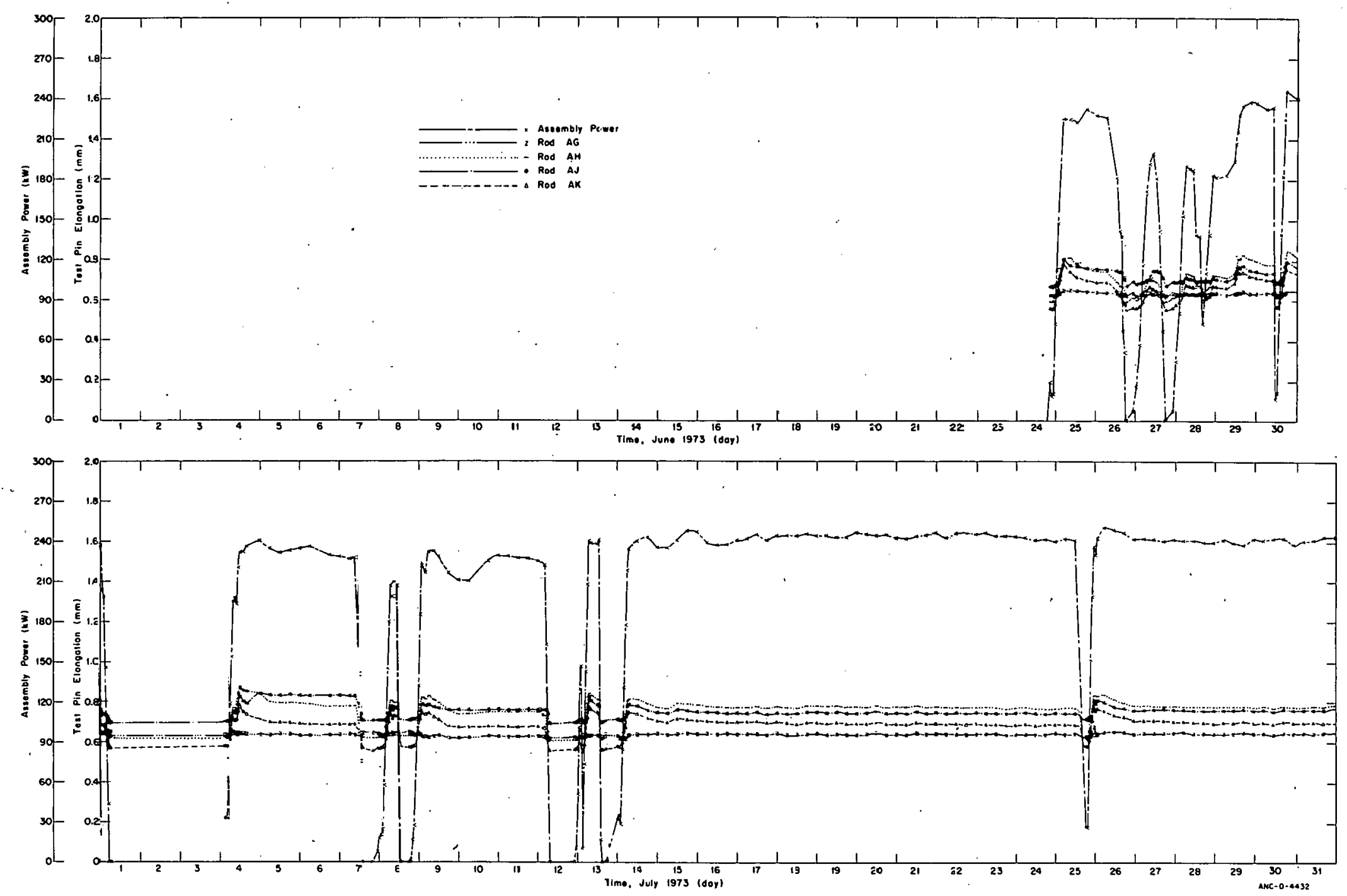

Fig. IV-43 Elongation history of IFA-226 test pins from June 1973 through July 1973. 


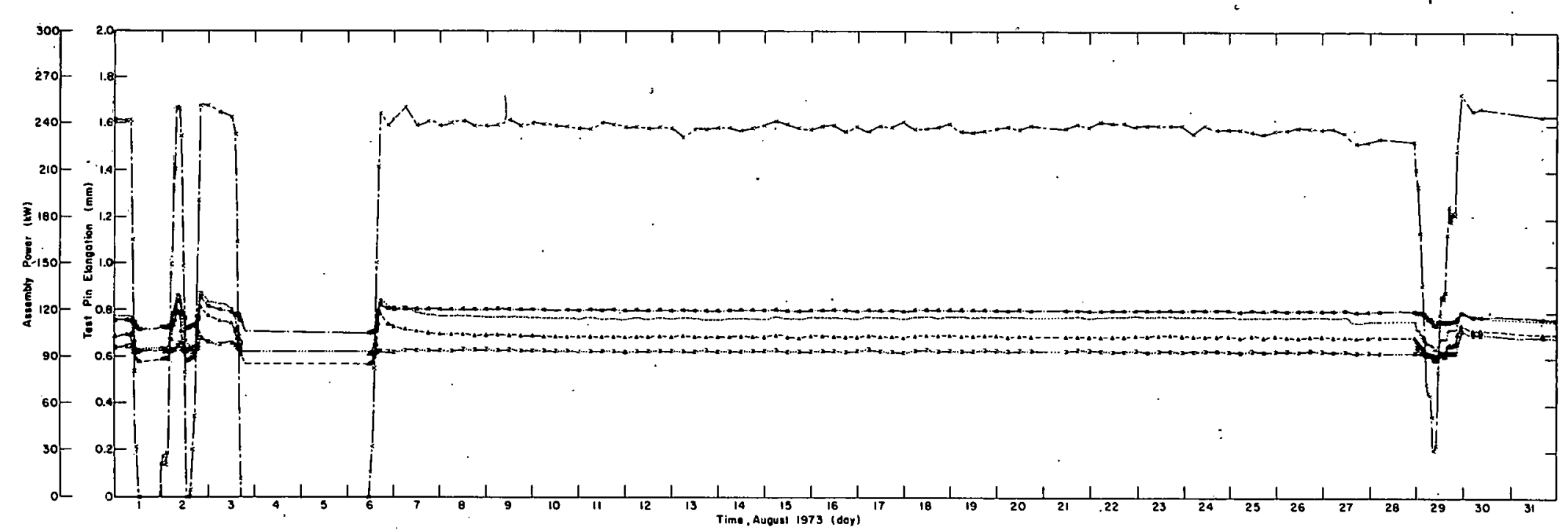

a

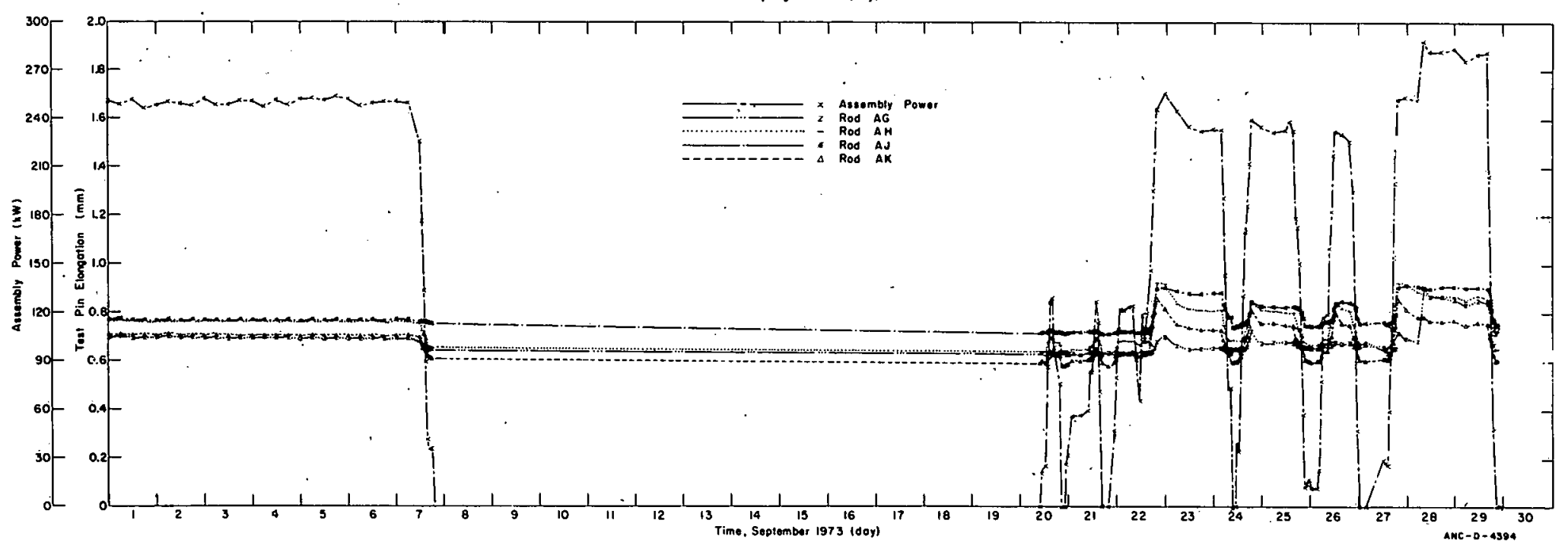

Fig. IV-44 Elongation history of IFA-226 test pins from August 1973 through September 1973. 

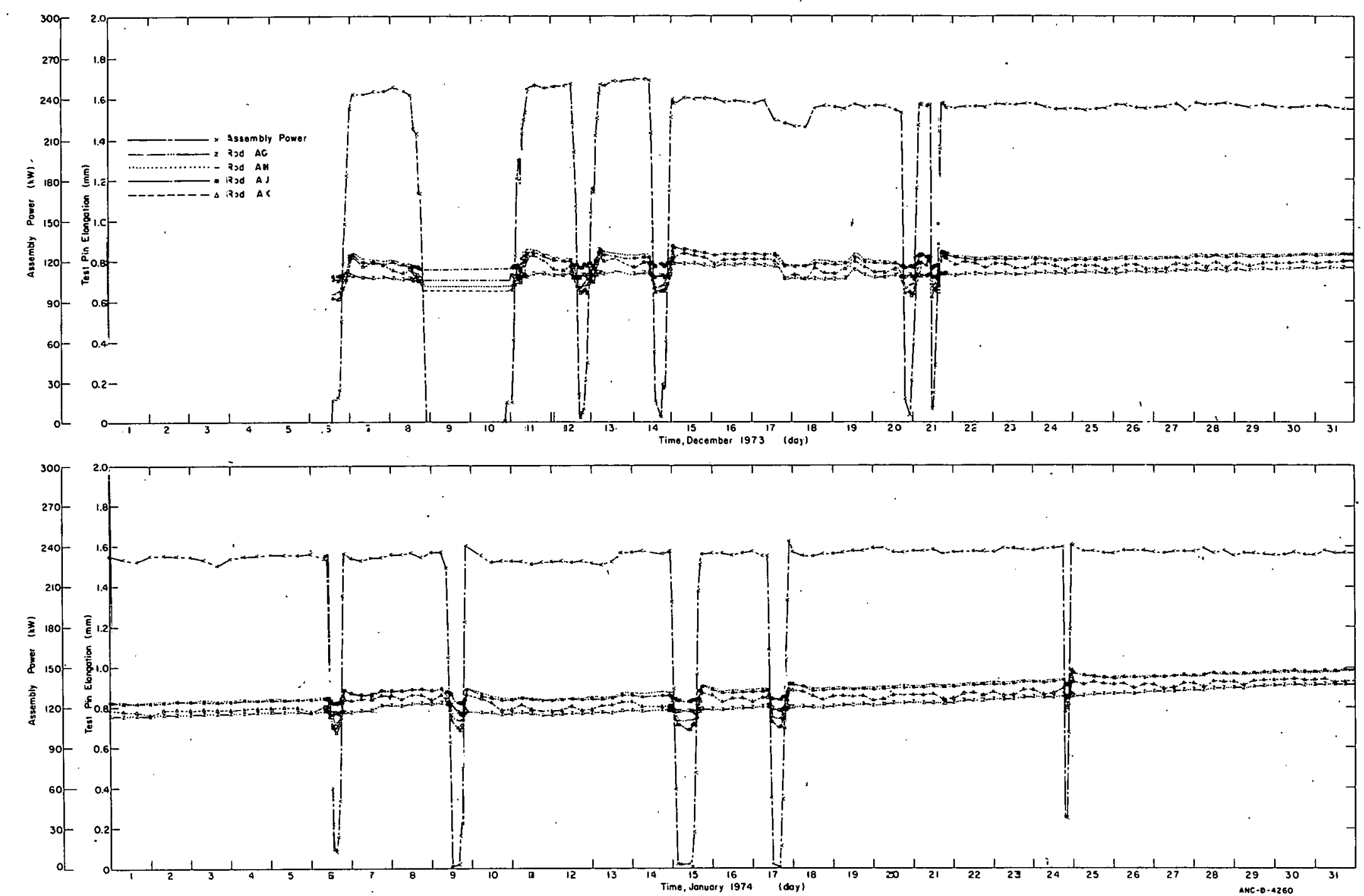

Fig. IV-45 Elongation history o: IFA-226 test pins from December 1973 through January 1974. 

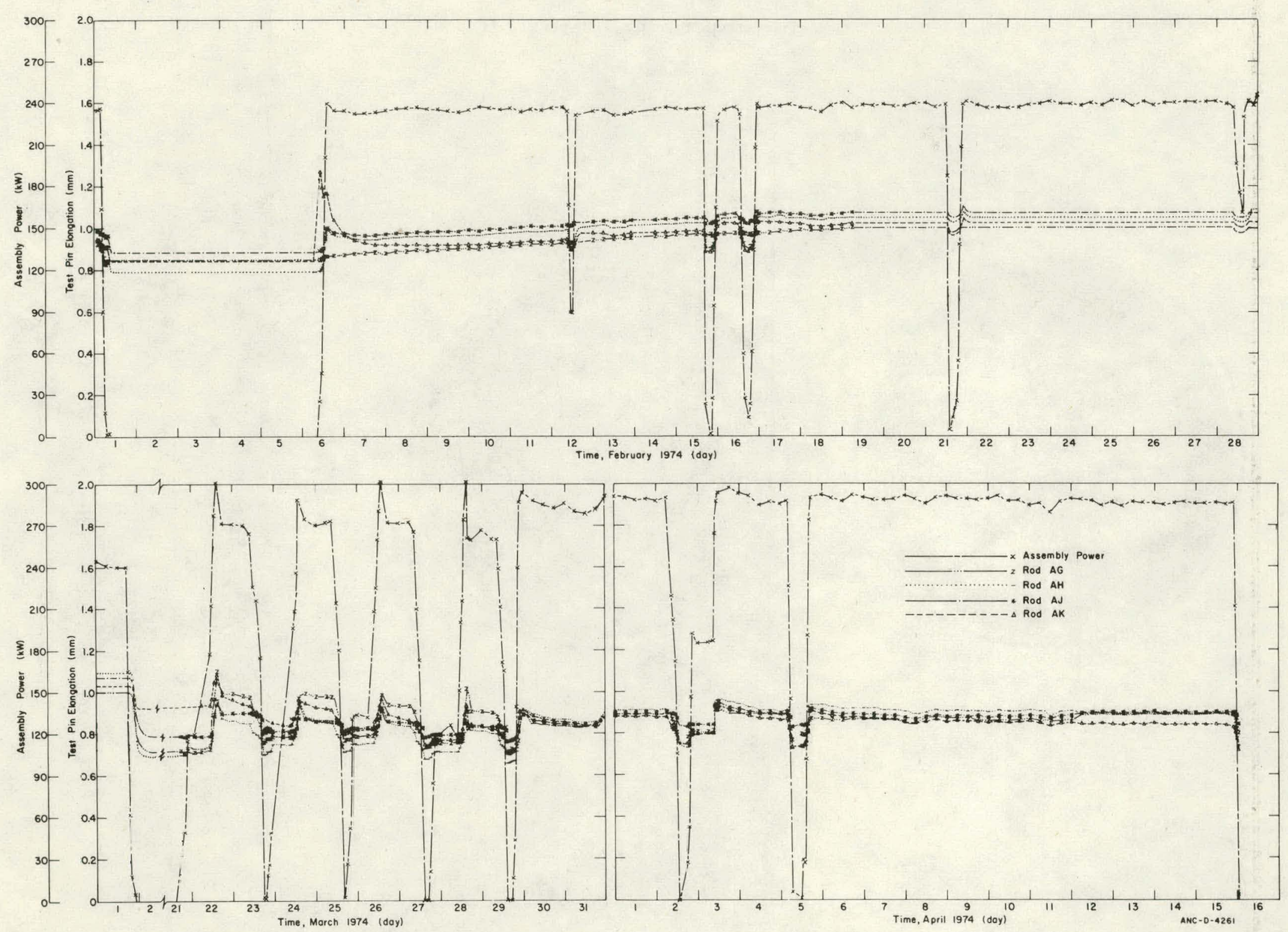

Fig. IV-46 Elongation history of IFA-226 test pins from February 1974 through April 1974. 


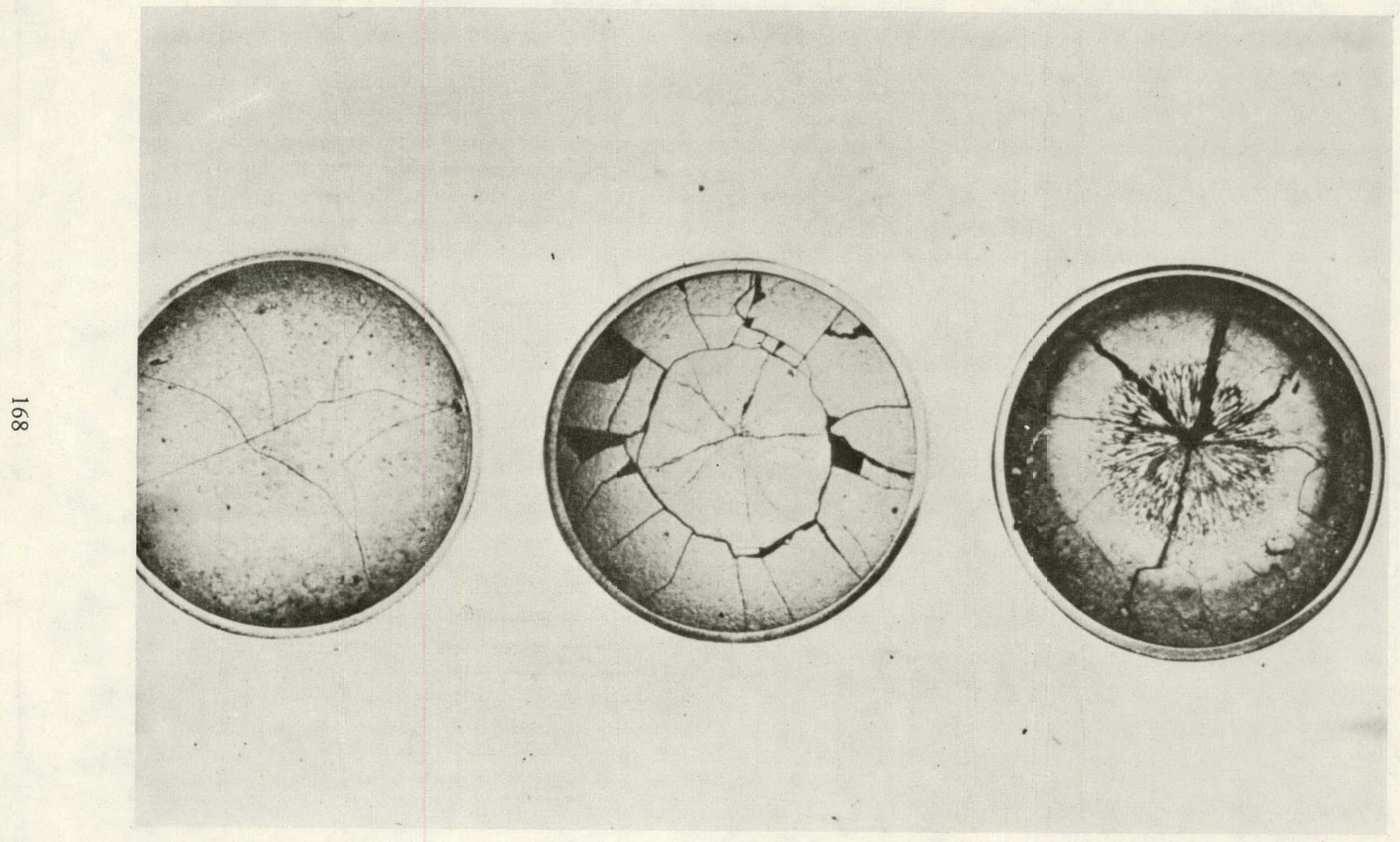

Fig. IV-47 Fuel rod cross sections showing pellet cracking. 


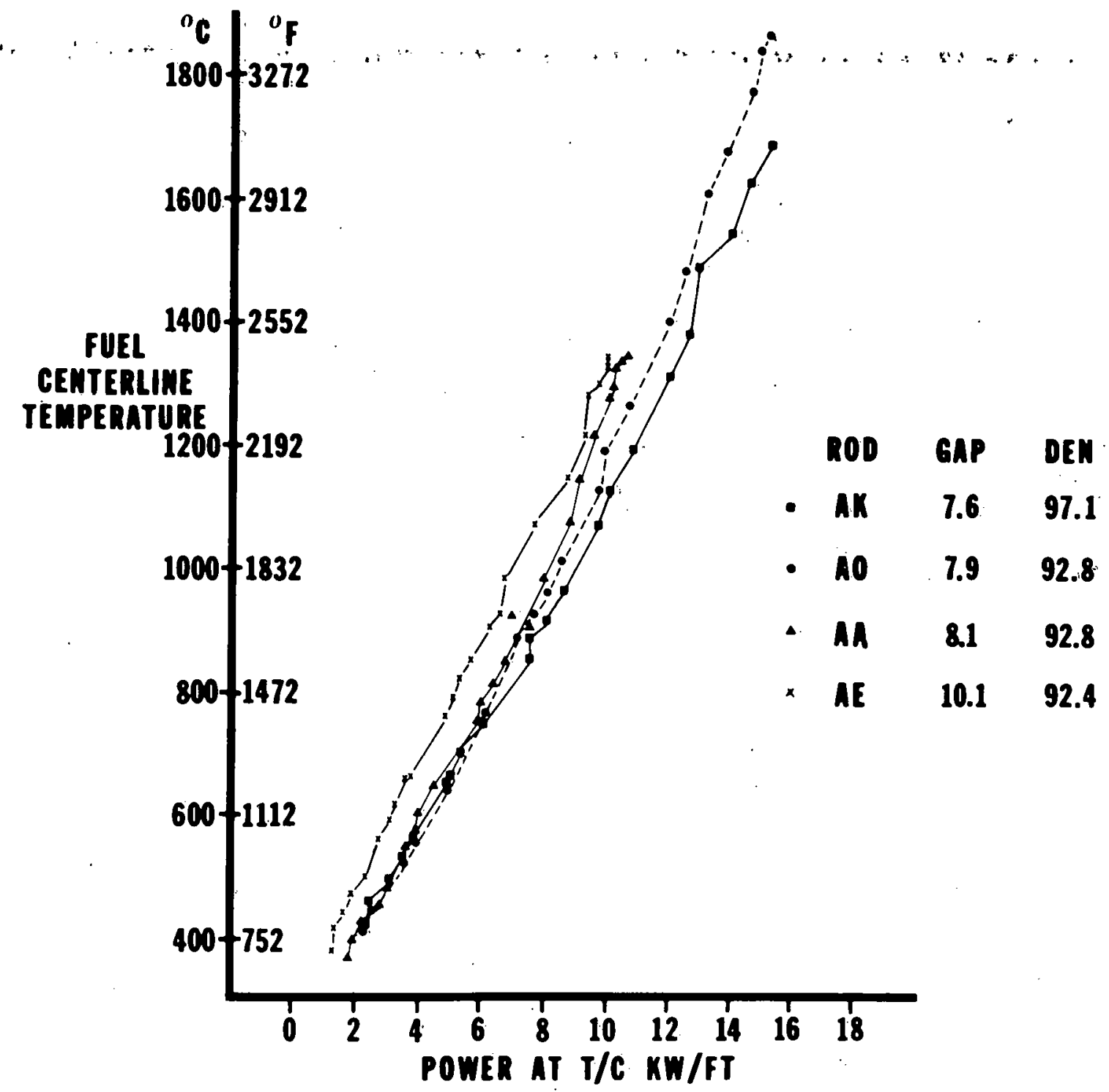

Fig. IV-48 Fuel centerline temperature vs. power during start-up.

Figure IV-49 represents the IFA-226 pellet centerline temperature versus power data for these same rods taken during the following 25-hour period (nearly full power for 9 hours, decreasing power for 16 hours). This figure suggests considerable pellet cracking and relocation. Note that centerline temperatures for the large pellet to cladding gap rod AE are now similar to the standard rod $\mathrm{AO}$ and $\mathrm{AA}$ temperatures. The high-density rod $\mathrm{AK}$ temperatures remain somewhat lower than $\mathrm{AA}, \mathrm{AE}$, and $\mathrm{AO}$, the relative difference being clearer at high heat ratings.

Pellet cracking and relocation effects become more apparent in Figures IV-50, IV-5.1, and IV-52. Figure IV-50 represents high-density rod AK data for the first power ramp. Note that the increasing power data is continuous and nearly linear while the decreasing data is irregular, suggesting dimensional changes within the pellet. Note the hysteresis effect at 10 to $1.4 \mathrm{~kW} / \mathrm{ft}$, possibly due to cracked pellet healing at high temperatures, followed by 


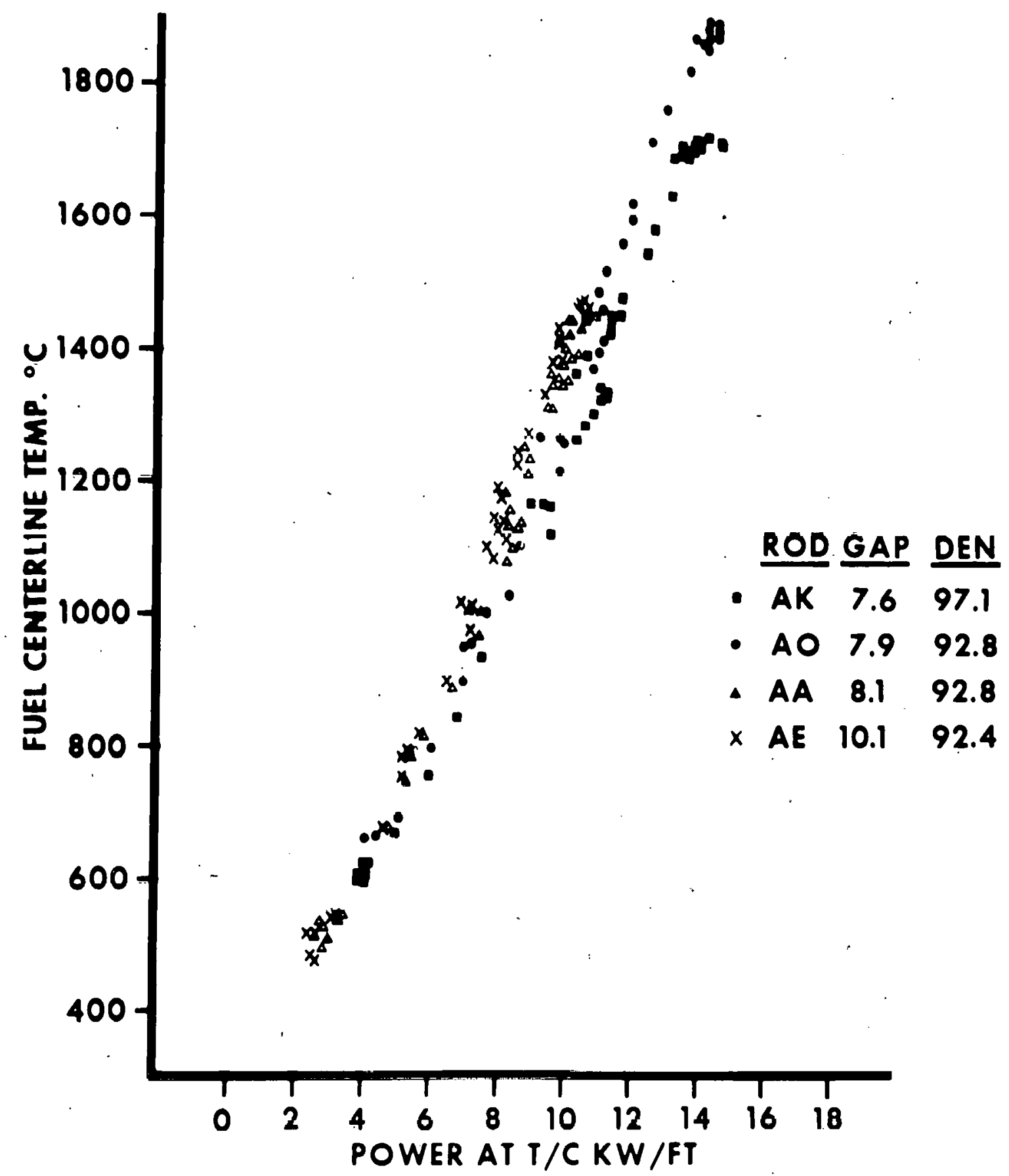

Fig. IV -49 Fuel centerline temperature during 25 -hour period following start-up.

reformation of the pellet-to-cladding gap upon subsequent power reduction, followed in turn by the reinitiation of pellet cracking during the temperature changes at lower power levels when the fuel is relatively stiff. Figures IV-51 and IV-52 compare the fuel centerline temperature data during the first four full power ramps. It appears after four power ramps that the pellet cracking, and therefore the thermal response of the rod, has stabilized. 


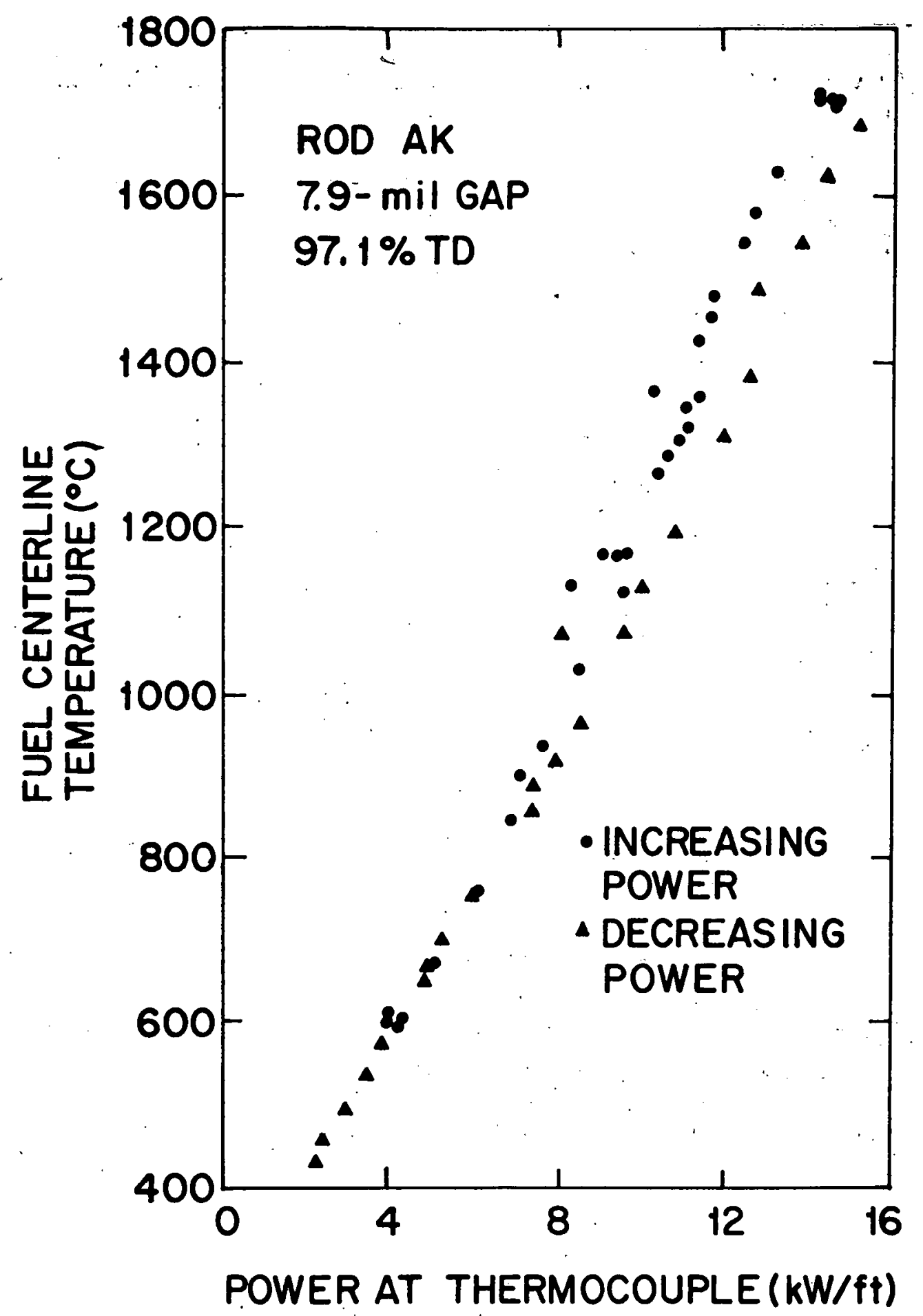

Fig. IV-50 Fuel rod AK centerline temperature vs. power for first power ramp.

Figures IV-53 and IV-54 compare the measured fuel centerline temperatures versus power data at various burnup levels. It appears that the fuel temperature decreases slightly with burnup and then increases slightly until, at 15,000 MWD/MTU burnup, they are approximately the same as measured at beginning of life. It is also apparent that the variability of the temperature response at high burnup is less than that measured during the first four power ramps at beginning of life (when there may have been more fuel pellet fragment movement). 


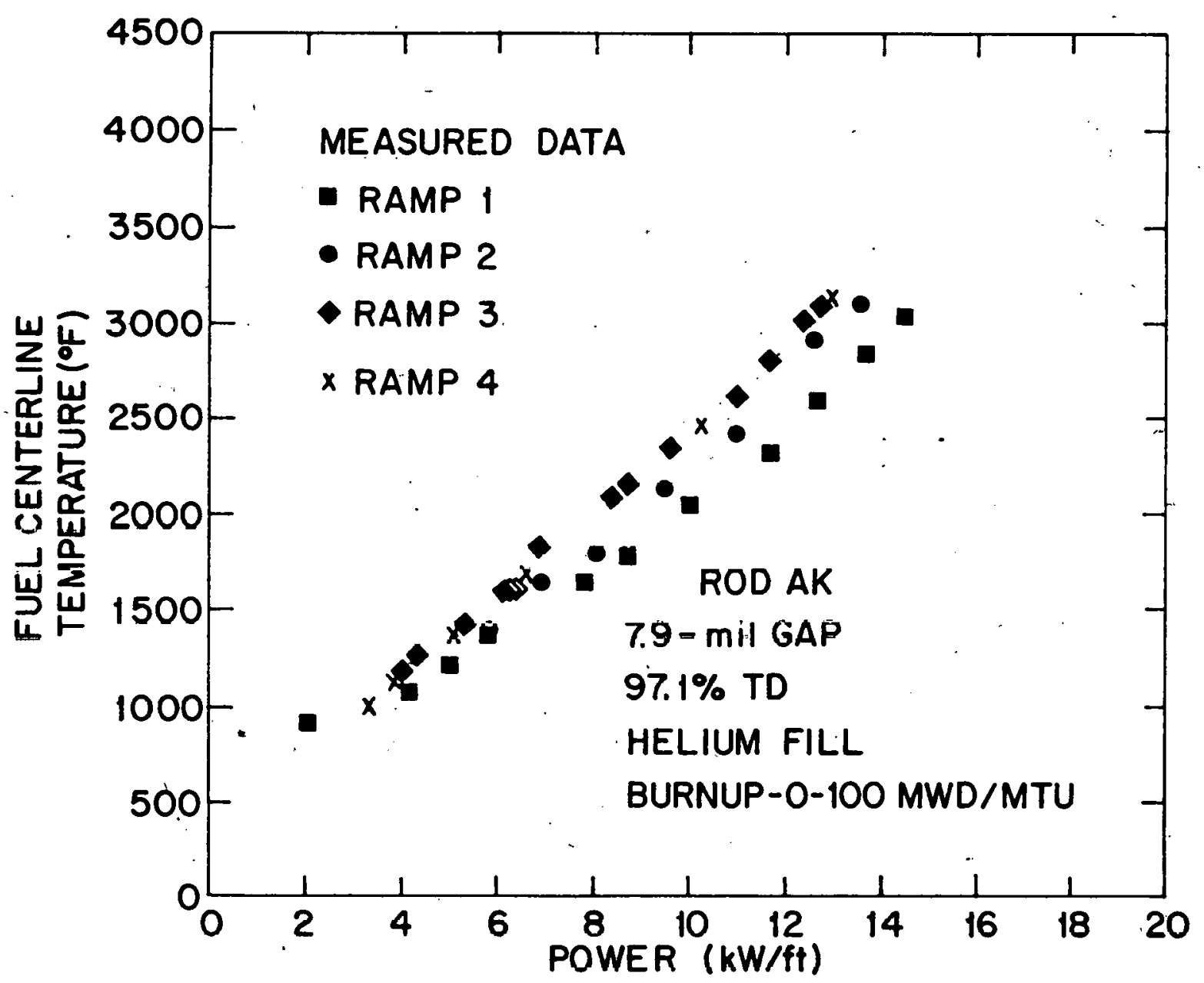

Fig. IV-51 Rod AK fuel centerline temperature for first four full power ramps.

4.2.3 Fuel Rod Pressure Data. Figures IV-55 and IV-56 present the IFA-226 fission gas pressure data at full power as a function of time. Very little pressure increase was observed until after approximately 15 months of irradiation, and the various fuel design variables did not strongly affect the fission gas release processes. 


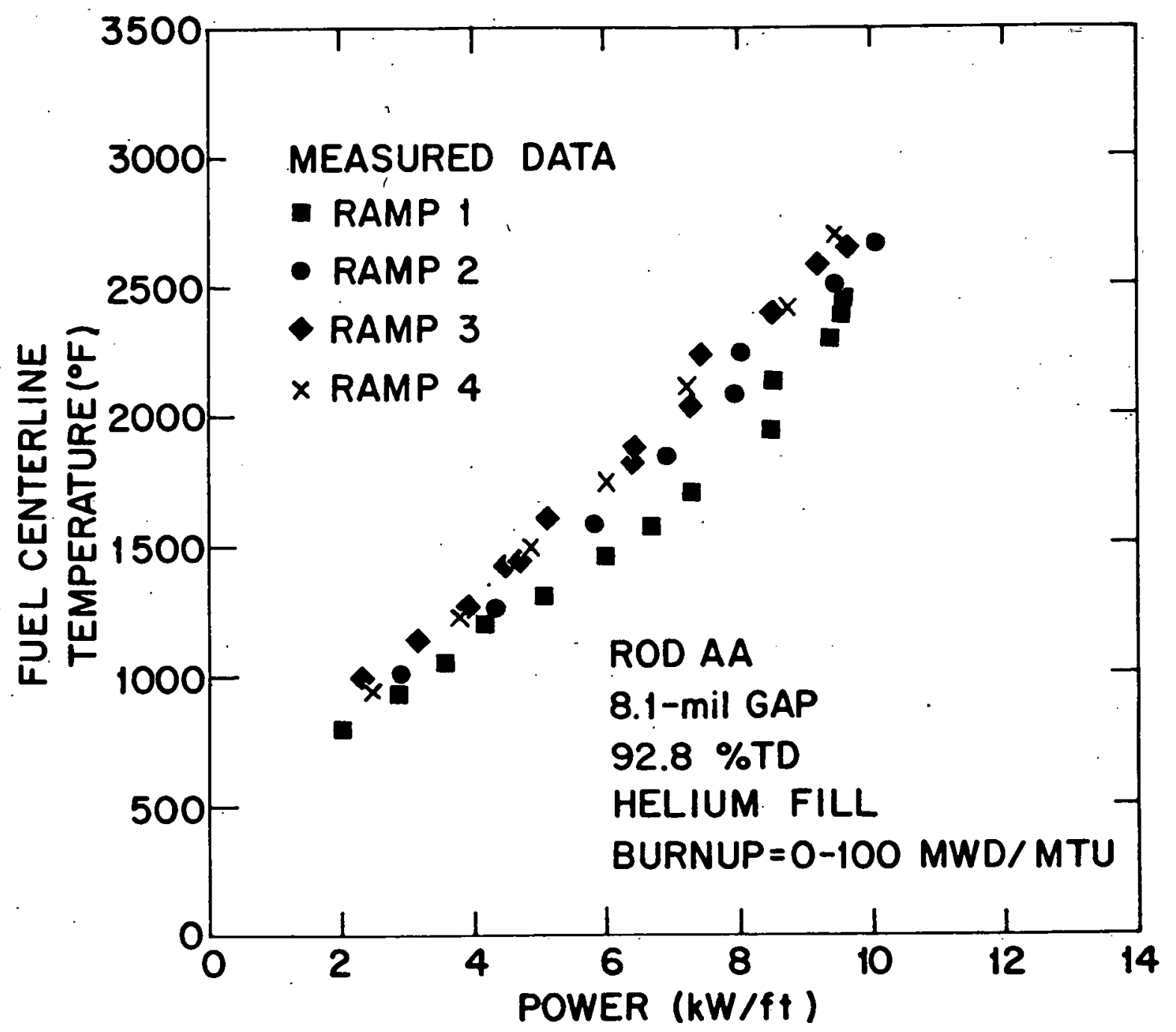

Fig. IV-52 Rod AA fuel centerline temperature for first four full power ramps. 


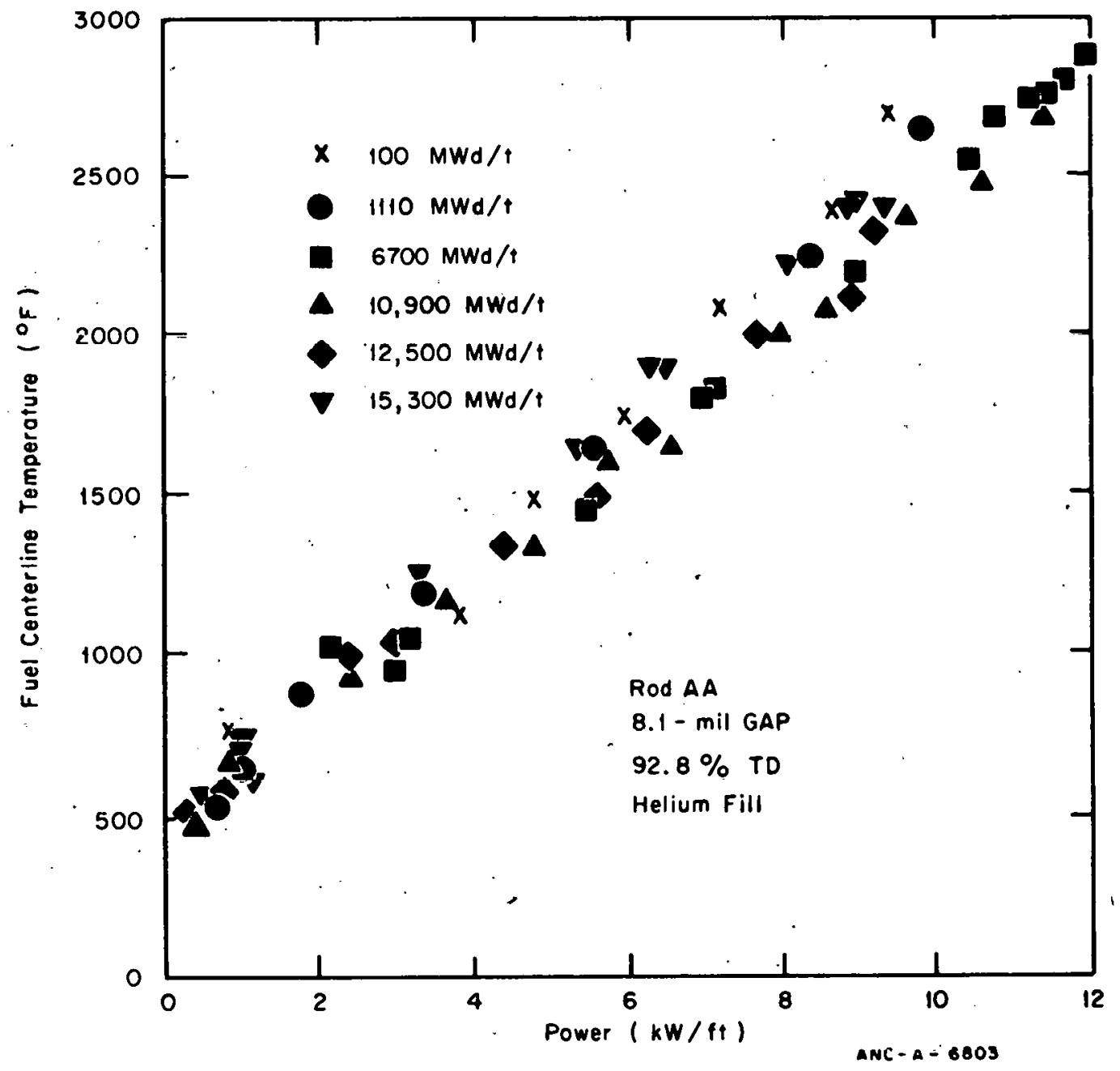

Fig. IV-53 Fuel rod AA centerline temperature vs. power at extended burnup levels. 


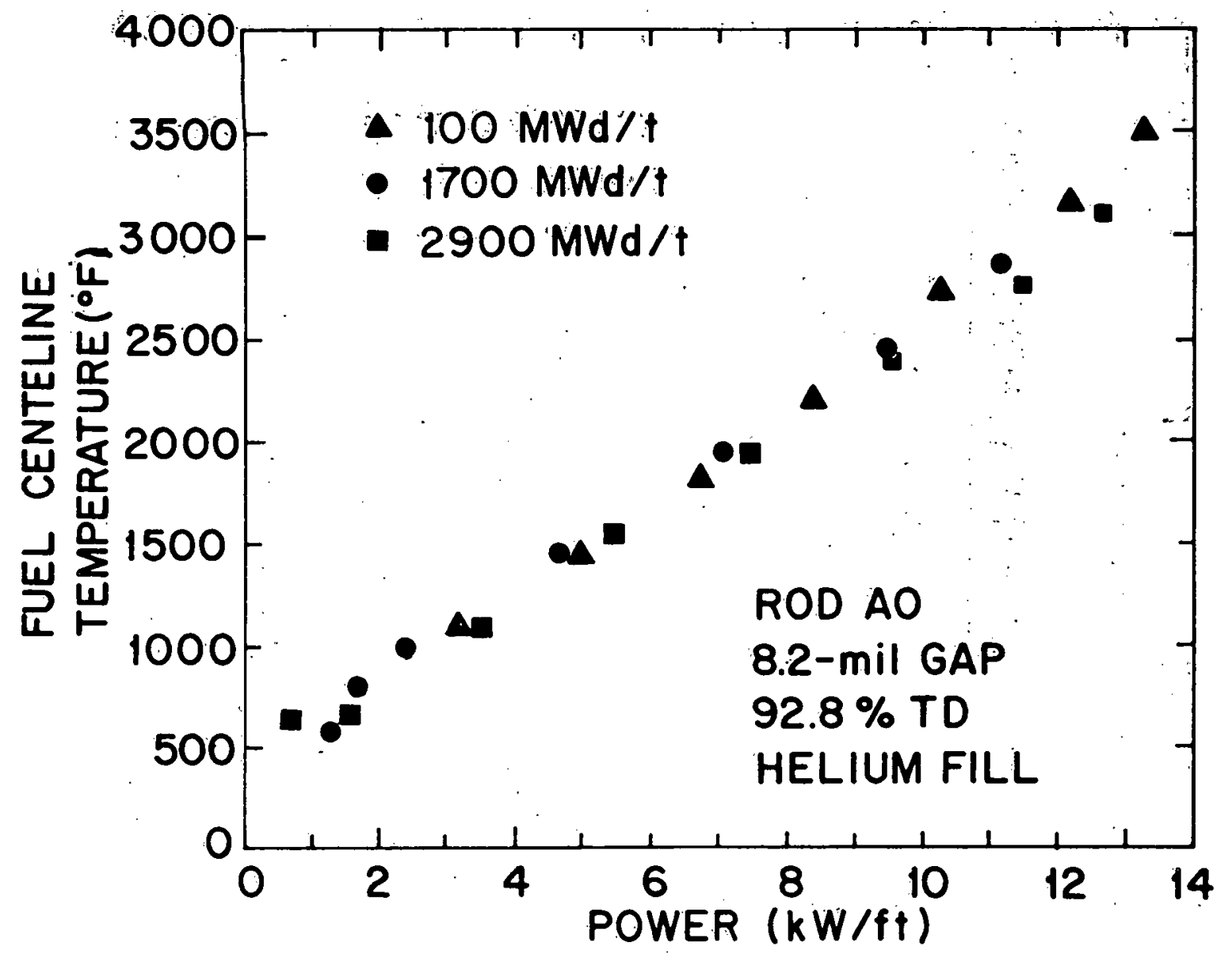

Fig. IV-54 Fuel rod AO centerline temperature vs. power at extended burnup levels. 


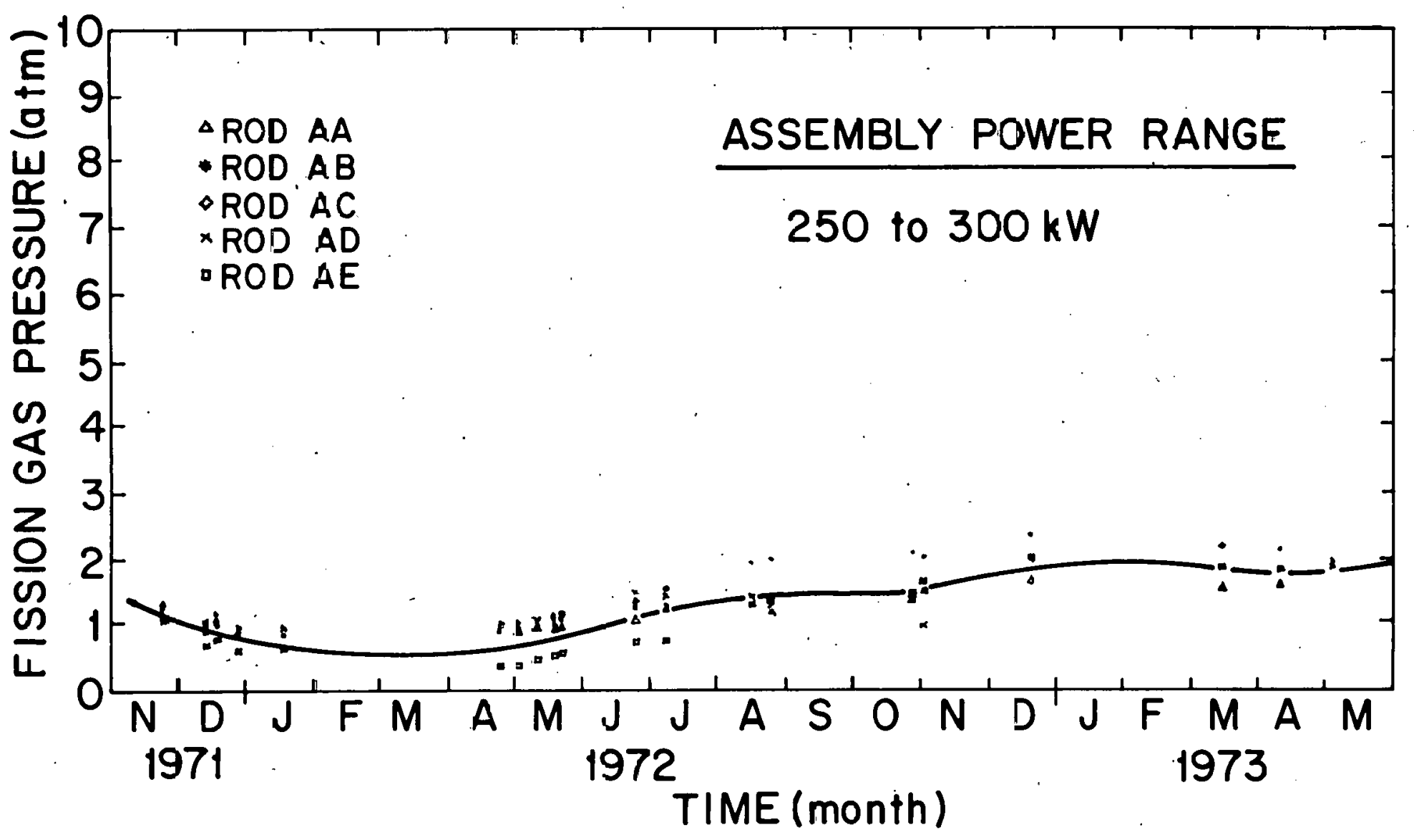

Fig. IV-55 Fission gas pressure history (1971-1973) 


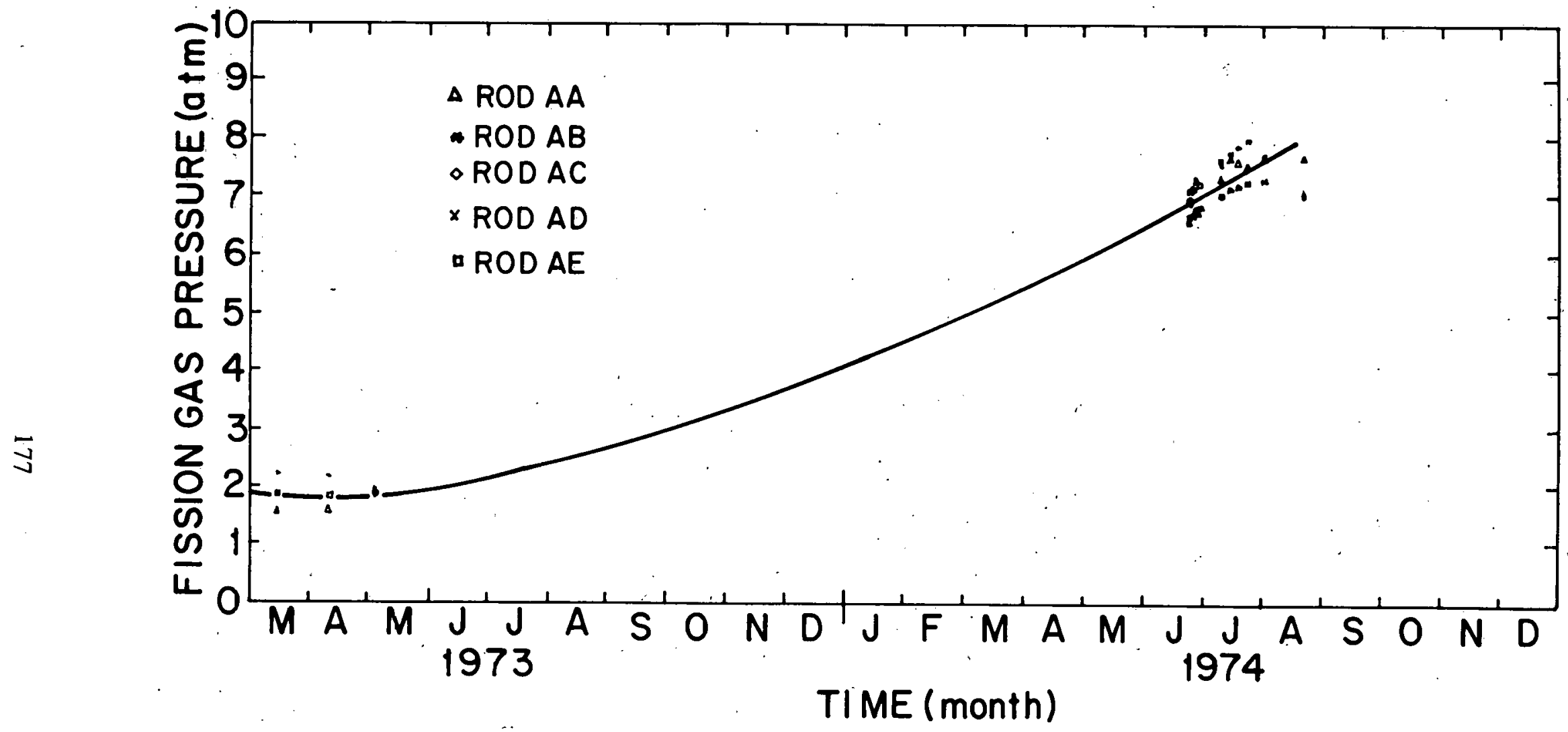

Fig. IV-56 Fission gas pressure history (1973-1974). 


\subsection{FRAP-S1 Model Verification \\ D. R. Coleman}

Several types of data-prediction comparisons as well as model sensitivity results are reported to verify capabilities of FRAP-S1. This computer model comprises the initial version of a steady state Fuel Rod Analysis Program (FRAP) being developed to support reactor safety analysis. Primary application is in supplying initial conditions for the transient response model,' FRAP-T.

Analytical comparison between annular and cracked pellet gap conductance models showed more thermal conservatism in the annular model applied to standard PWR and BWR design analyses, especially for the unpressurized BWR rods. Use of different thermal models had large influcncc on key pressurc, gap, and stored energy conditions, the effect increasing over the extended burnup periods specificd in thesc cascs.

Comparing results from both annular and cracked-pellet gap conductance models with available data showed bettcr fuel tempcrature agreement using the cracked-pellet model for 60 and $80 \%$ of the startup and burnup data points, respectively. This conclusion is somewhat affected by empiricism in the model since development and verification data sets have some rods in common, and has not been verified for pressurized rods. In order to match the apparently better gap conductance model with design conditions most reflected in the data sample, however, the remaining comparisons did use the cracked-pellet model.

Early-life fuel thermocouple comparisons for unpressurized rods showed some tendency to overpredict centerline temperature once a small amount of gas release occurred. The temperature agreement improves with burnup, however. Beyond initial startup, calculated thermal conditions are within data uncertainty $\left( \pm 200^{\circ} \mathrm{C}\right)$ of the measured values.

The hcat transfer increasc predicted for helium preseurized rods has not been supported by comparisons with limited data and design code results. More data are needed for these conditions. There are indications that actual pressure and gas composition effects have less influence on gap heat transfer than predicted.

For fuel temperature measurements as a whole, sufficient account has not been taken of decalibration and transmutation effects. Currently, at least some degree of adequacy can often be shown, rcgardlcss of what model is used due to scatter and lack of measurement reproducibility. It is the likelihood of stochastic processes which accounts for current emphasis on a maximum sample size approach for verification of fuel behavior models. A total of 270 rods was used for the current data comparison study.

Many melt radius comparisons lost definition at incipient melt conditions due to coarse interior nodalization built into the model. Otherwise, the higher power results are generally within measurement range of the data with some indication of a few percent conservatism in the integrated fuel thermal conductivity at higher temperature. 
Gas release data comparisons showed better model capability for steady power operation between 7 and $10 \mathrm{~kW} / / \mathrm{ft}$. Otherwise, the comparisons suggest need for a more mechanistic model. The current predicted sensitivity to fuel temperature alone seems to be too dominant. Here, interpretation is susceptible to, uncertainty in input power histories used to model the experiments. Release fraction is underpredicted most often at lower temperatures when recoil/knockout and cracking effects should dominate. Some comparisons indicated a power cycling or discontinuous gas release effect currently not modeled. Other data indicated higher influence of burnup than predicted by the model whose release probability is not affected by cumulative fuel damage or buildup of interconnected porosity. The evidence is that for thermally driven programs, thermal and gas release models should not be independently fine-tuned.

Rod internal pressure comparisons show more capability to model startup pressure changes in rods with considerable plenum volume. This behavior suggests the need to modify gas temperature and volume assumptions over the active length. Pressure increase with burnup will be sensitive to accuracy of the fuel temperature model. Small power differences at high fuel temperature controlled calculated pressure buildup to a greater extent than indicated by some of the data which showed more of a fuel density effect.

Fuel thermal expansion axially was well represented by the model for shoulder contact conditions in the pellet stack. Flat pellet axial expansion was overpredicted. Cladding axial expansion and small amounts of fuel deformation after gap closure are not now accounted for by the program ${ }^{[a]}$. With an annular gap mechanical interaction model, heat rating to initiate gap closure was overestimated.

Cladding circumferential strain after gap closure was better characterized below pellet temperatures associated with increased fuel plasticity. Currently there is no fuel deformation model other than thermal expansion and swelling. The range of cladding local hoop strain for instrumented rods was surprisingly well accounted for once gap closure was predicted. Since the model does not account for negative fuel deformation ${ }^{[b]}$, cladding strain at yield may have been overestimated.

In rods operated up to 18,000 hours at PWR conditions, cladding diameter decrease from compressive creep strain was predicted to occur when measured. Circumferential strain agreement in moderate- to high-power rods with more incidence of hard gap closure could not be analyzed within accuracy of the pressure model and input power history. Adequacy of a simplified fast flux creep rate acceleration model ${ }^{[b]}$ was not determined in low power rods with less gap closure for the same reason. Opposition of mechanical interaction and cladding creepdown effects confounded interpretation of many comparisons using available Postirradiation Examination (PIE) hoop strain data. The current deformation and thermal expansion models seem most applicable to conditions involving uniform strain mechanisms.

[a] Improved FRAP-S2 model completed without coupling.

[b] Improved MATPRO properties completed without coupling. 
Quantitative results of comparisons with zircaloy corrosion and hydrogen pickup data were not determined due to lack of information on as-built conditions. Cladding temperature and operating time have strong influence on both models. For corrosion buildup, an acceleration factor adjusting lab correlations for in-pile application seems somewhat arbitrary, overlapping with what may actually be a temperature or coolant chemistry effect. Both models do predict cumulative effects impacting initial conditions for accidents involving high burnup rods. Cladding surface condition and mechanical properties would be involved. Qualitatively, the models seem ready to pass information to the transient code if material property coupling existed. The predictions are usually within the measurement range.

Standard design analyses for full-size rods were done to establish typical ranges for FRAP-S1 output parameters expected to impact the transient code. Design and correlative input parameters were varied within realistic limits using a Monte Carlo technique. The resulting output variation was compared to that obtained by changing power histories over a range representing 80 to $90 \%$ of the core, the core average rod being the nominal case. End-of-life burnup was the same for all standard design runs. Results showed large burnup effects on key initial conditions for off-normal analysis. Initial thermal condition was more strongly controlled by heat-rating which supports the often used hot channel assumption. Gas content, gap size, cladding strain, helium fraction, void volume, and rod surface conditions, however, were more affected by variation in design and operating parameters. The highest power rod then is not necessarily the limiting case for mechanical interaction or rod ballooning; there seem to be many more rods whose conditions lie inside the lead rod distribution than originally thought. These results can be subsequently used for FRAP-T sensitivity studies of accidents at varying burnups.

Full details of the FRAP-S1 model verification effort have accompanied transmittal of the program to Argonne Code Center.

\section{LOCA ANALYSIS VERIFICATION}

\subsection{PWR Uncertainty Analysis Methods Study-12-Node Plant Model}

M. E. Wells

A statistical methods study has been initiated to investigate the advantages of several statistical approaches to code uncertainty analysıs. The methods study uses a RELAP4 model of a PWR containing a small number of nodes. Uncertainties in a key group of parame ters are considered during the study. The optimum statistical method determined by the methods study is intended for use in a RELAP4-BE code uncertainty analysis with a more detailed PWR model and complete set of the most influential LOCA parameters.

The ground rules for model development and parameter selection for the statistical methods study are as follows: (a) the methods study is run on a modified RELAP4/Mod$5 /$ Version 26 code; (b) performance predictions are computed during the first 20 seconds of 
blowdown; (c) a $200 \%$ cold-leg break on a Westinghouse Trojan plant is used; (d) break size, break location, and peak power are fixed for the statistical methods study, and the study is therefore based on a specified, rather than a random, accident;. (e) code parameters reflect both input and code correlation uncertainties. The parameters provide a typical crosssection of the many possible variables in the prediction of LOCA effects on a typical PWR.

The minimum node módel developed for this task (Figure IV-57) uses 12 volumes, 16 junctions, and 7 heat slabs and represents the best compromise between accuracy and running time. Good agreements in general performance trends, such as rate of temperature rise, occurrence and level of temperature peaks, time of flow reversal and patterns of cooldown behavior, are found between this model and a detailed plant model used for LOFT typicality studies. A comparison of hot slab cladding temperature predictions using the detailed model and the simplified model is shown in Figure IV-58. As can be seen, the peak cladding temperatures agree within $20^{\circ} \mathrm{F}$ :

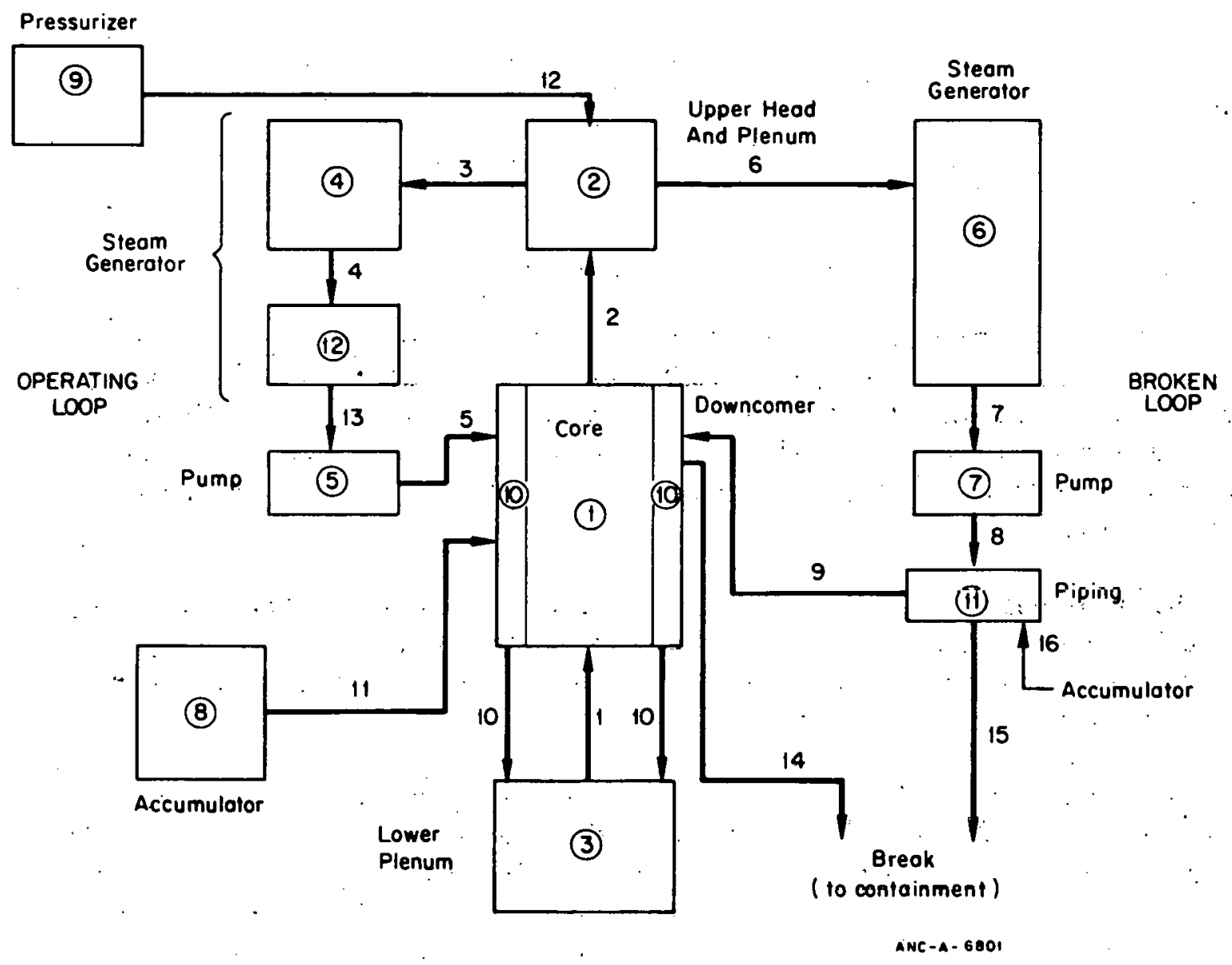

Fig. IV-57 Simplified Trojan plant model. 


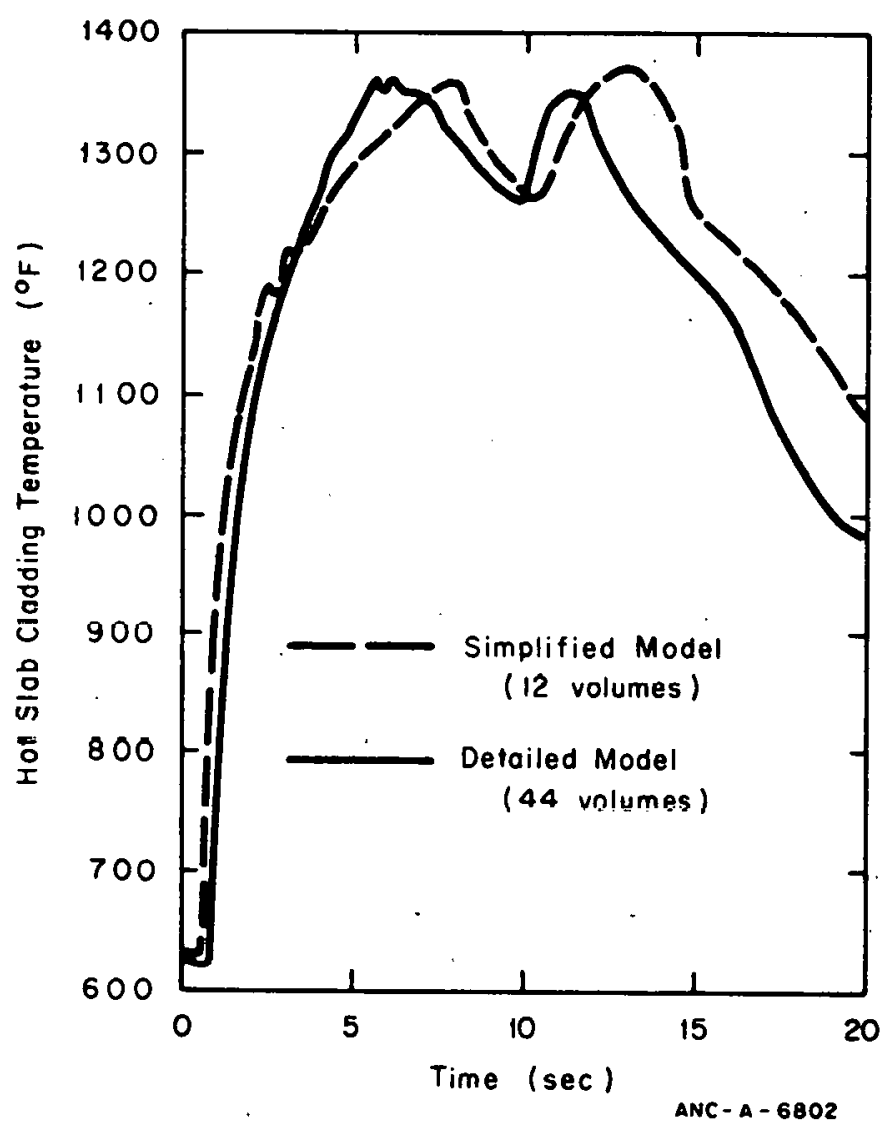

Fig. IV-58 Simplified plant model vs. detailed plant model.

Changes were made in the simplified model after the performance comparisons were completed to produce a base case for the statistical methods study. These changes incorporate the nominal bestestimate values so far established for the RELAP4-BE uncertainty analysis and also generally improve the best-estimate capability of the model. This base case modeled the first 20 seconds of blowdown in approximately 15 minutes of computer time.

Seven parameters were selected for variation in the statistical methods study. They are: differential discharge coefficients between the two break ends, power peaking factor for the hot channel, initial gap conductance, value of .CHF, post-CHF heat transfer coefficient, pump performance degradation, and decay heat.

Nominal, or best-estimate, values were selected for each variable, and uncertainty distributions about these nominal values were established. The uncertainties in three of the variables (power peaking factor, pump performance degradation, and decay heat) have already been determined for the RELAP4-BE uncertainty analysis. The uncertainties in the remaining four variables were estimated and are considered sufficient for the purpose of the me thods study. Further analysis is necessary to more rigorously determine these latter four uncertainty values for the RELAP4-BE uncertainty analysis. 
5.2 Comparison of RELAP4 Predictive Capability with Semiscale MOD-1 Core Test Data J. K. Meier

A necessary adjunct to the development of LOCA analysis codes is to verify the ability of these codes to predict PWR performance. One of the principal methods used to accomplish this verification is by data comparisons with the results of subscale tests such as Semiscale Mod-1. These comparisons fall into two categories: those performed with a model of the complete system and those performed with a single or small group of components. One of the components selected for data comparisons was the Semiscale Mod-1 core. The modeling approach taken in this study was to isolate the core and upper plenum from the rest of the Semiscale system, and, thereby, to assess the capability of RELAP4 in predicting core thermal-hydraulic response.

The model used for these analyses consisted of 15 volumes, 16 junctions, and 28 heat slabs. The core was divided into two channels with four volumes in each. Ten heat slabs were used in the hot channel of the core to represent the higher powered heater rods. Initial and boundary conditions were based on experimental data. The boundary conditions used at the bottom of the core were the inlet mass flow rate and fluid enthalpy. Boundary conditions used at the top of the core were the pressure and quality in the upper plenum.

Approximately 40 RELAP4 runs were conducted during this study in which a variety of modeling options were examined. The results of the most significant of these runs are summarized in Table IV-V. Figures IV-59 and IV-60 present a comparison of test derived cladding temperatures with RELAP4 predictions using models "base case I" and "change case II", as defined in Table IV-V. Base case I is essentially a standard RELAP4 model including the Berenson correlation ${ }^{[i \mathrm{~V}-24]}$. Change case II is the model which gave the most accurate prediction of core behavior in the studies.

Based on these runs, it was concluded that a Semiscale Mod-1 core model using RELAP4 Mod-4, version 15, could predict hot spot cladding temperature from Tests S-02-2 and S-02-3 with a reasonable degree of accuracy, provided the Berenson heat transfer correlation is replaced with a free convection correlation. Other conclusions and recommendations of this study are summarized below.

(1) The Berenson pool boiling correlation [IV-24] should not be used under high void fraction conditions. (Future RELAP4 heat transfer packages will not use Berenson in these conditions.)

(2) A heat transfer coefficient of $25 \mathrm{Btu} / \mathrm{hr}-\mathrm{ft}^{2}{ }^{2}{ }^{\circ} \mathrm{F}$ (representing free convection heat transfer) did a reasonable job of matching test data under high void fraction conditions.

(3) Using the local fluid conditions culculated by RELAP4, the Groeneveld 5.9 flow film boiling heat transfer correlation [IV-25] more accurately predicted Semiscale Mod-1 core behavior than did the Dougall-Rohsenow correlation [IV-26]. 


\section{TABLE IV-V}

SUMMARY OF COMPARISON OF RELAP4 PREDICTIONS WITH

SEMESCALE MOD-1 CORE TEST DATA

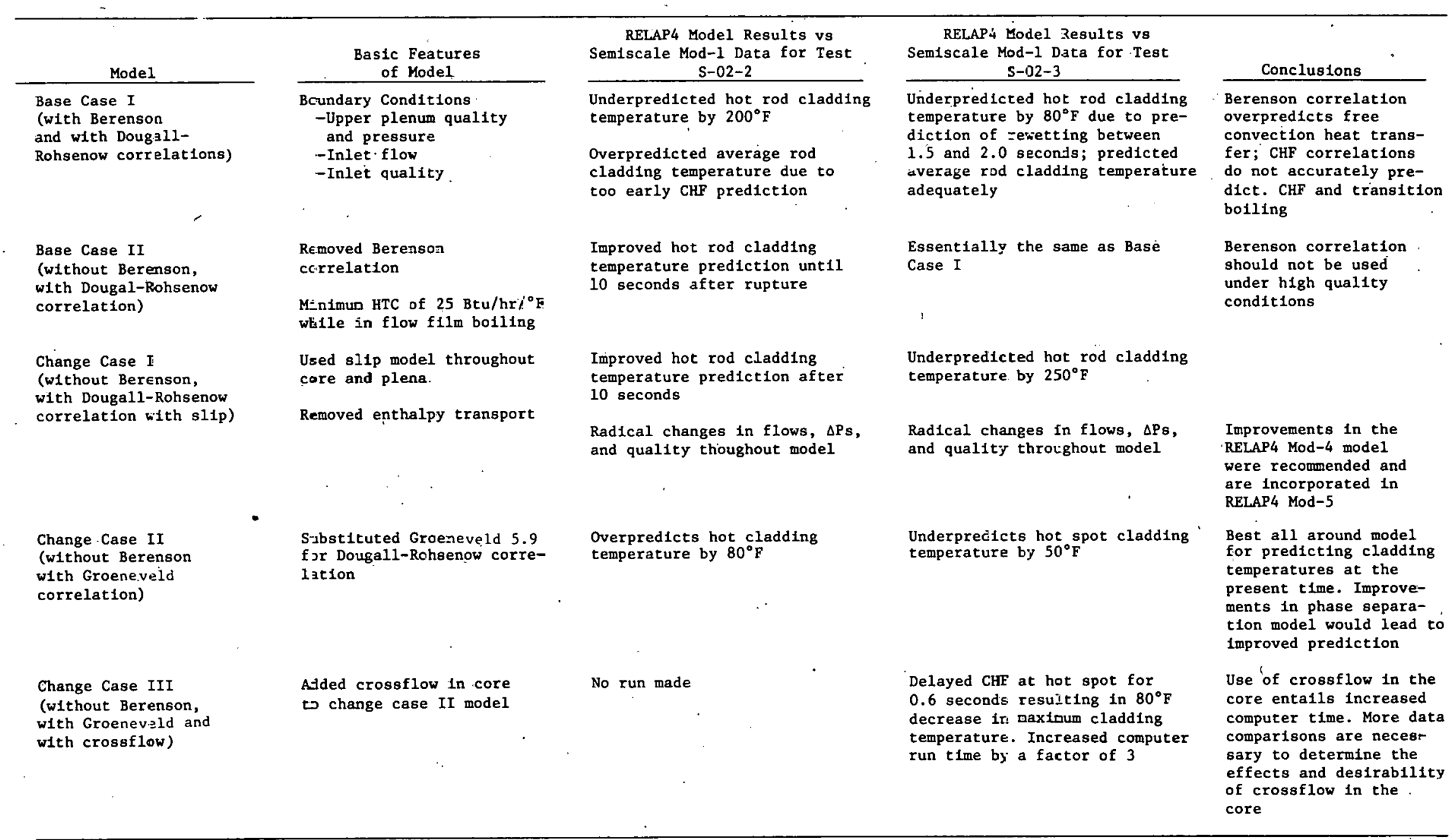




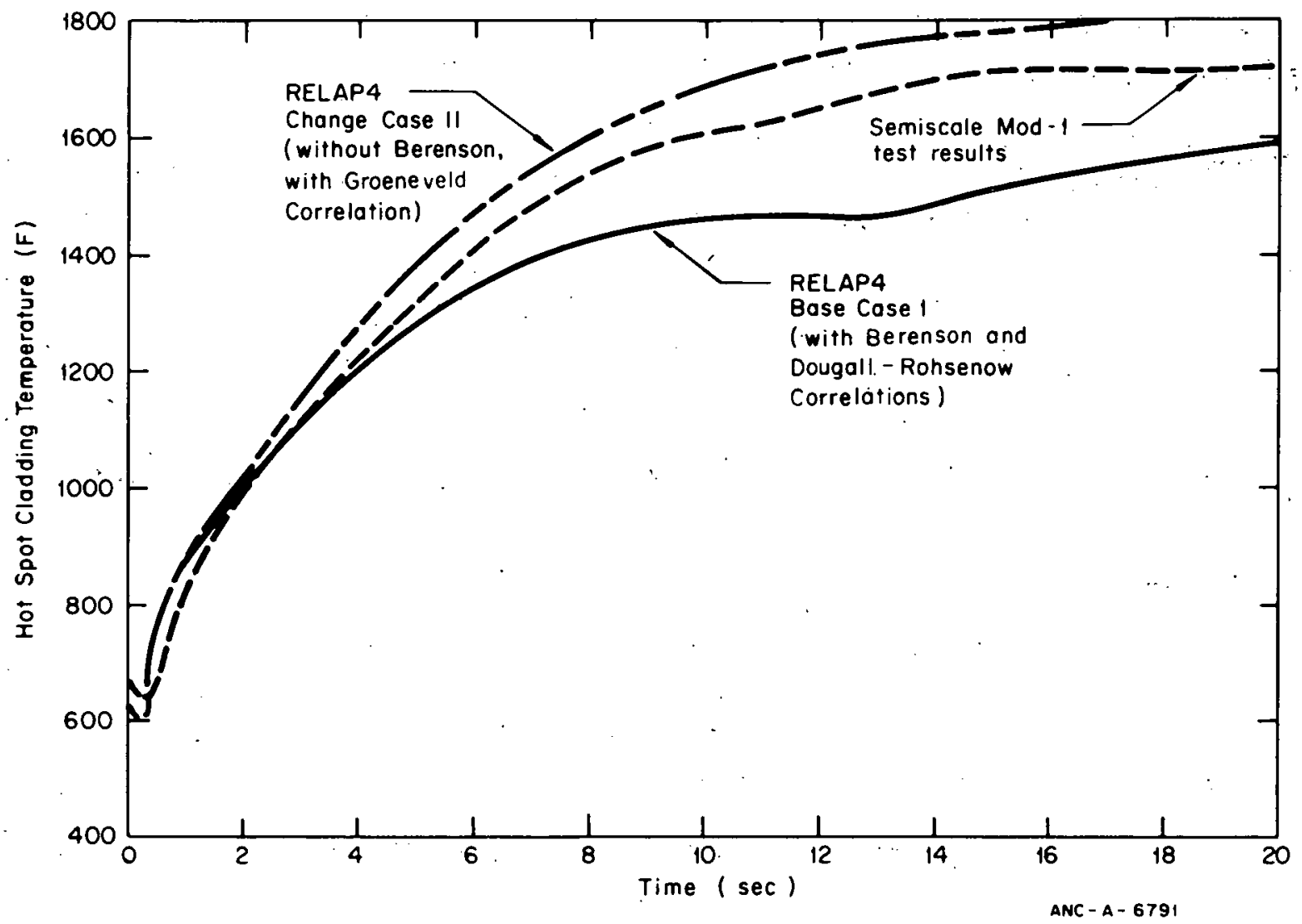

Fig. IV-59 Correlation of LOCA analysis code predictions with Semiscale Mod-1 Test S-02-2 results.

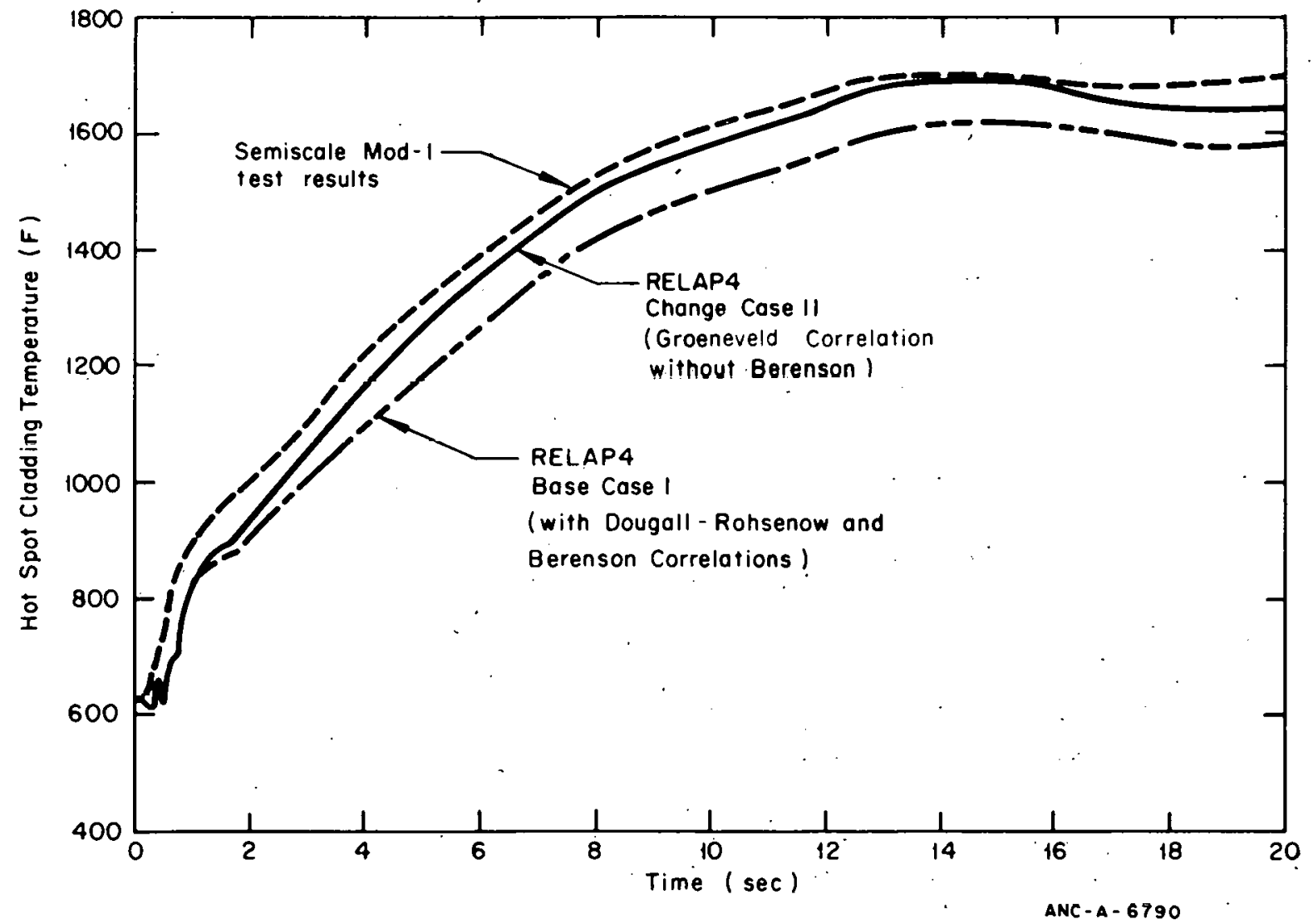

Fig. IV-60 Correlation of LOCA analysis code predictions with Semiscale Mod-1 Test S-02-3 results. 
(4) Both hot and average channels are necessary to model the Semiscale core.

(5) Development of improved phase separation and critical heat flux models would improve the analysis of core behavior.

(6) There is a need for an improved data base for low mass flux, high quality flow film boiling heat transfer correlations.

(7) Density measurements inside the Semiscale Mod-1 core and upper plenum would allow more complete code verification at the component level.

(8) When a separate modicl of the core is constructed, use ot upper and lower plenum pressures as the principal boundary conditions inay lead to scriously incorrect predictions of core flow rates due to inaccuracies in the phase separation models. If' the inlet flow to the core is used as the principal boundary condition, these inaccuracies will he minimized.

(9) Evaluation of the RELAP4 core model should continue using new Semiscale Mod-1 test data and improved versions of the code as they become available.

\section{REFERENCES}

IV-1. B. E. Launder and D. B. Spalding, Mathematical Models of Turbulence, New York: Academic Press, 1972.

IV-2. M. C. Chaturvedi, "Flow Characteristics of Axisymmetric Expansions", Journ. Hydraul: Div. A.S.C.E. $89 m$ (HY3) (1963) pp 61-92.

IV-3. N. J. Lipstein, A.S.H.R.A.E.J. 4 (1.962) pp 43-47.

IV-4. R. G. Teyssandier and M. P. Wilson, "An Analysis of Flow Thruugh Sudden Enlargements in Pipes", $J$. Flutd Mech., 64 (1974) pp 43-47.

IV-5. A. J. Ede, C. I. Hislop, R. Morris, "Effect on the Local Heat Transfer in a Pipe of an Abrupt Disturbance of the Fuid Flow: Abrupt Convergence and Divergence of Diameter Ratio 2:1", Proc Inst. Mech. Eng., 170 (1956) p 1113.

IV-6. R. A. Seban, A. Emery, A. Levy, "Heat Transfer to Separated and Reattached Subsonic Turbulent Flows Obtained Downstream of a Surface Step", J. Aero/Space Sci, 26 (1959) p 809. 
IV-7. D. B. Spalding, "Heat Transfer from Turbulent Separated Flows", J. Fluid Mech., 27 (1967) pp 97-109).

IV-8. D. O. Hobson and P. L. Rittenhouse, Deformation and Rupture Behavior of Light-Water Reactor Fuel Cladding, ORNL-4727 (October 1971).

IV-9. D. G. Hardy, "High Temperature Expansion and Rupture Behavior of Zircaloy Tubing", Topical Meeting on Water Reactor Safety, American Nuclear Society, Salt Lake City, March 26-28, 1973, CONF-730304.

IV-10. C. C. Busby and K. B. March, High Temperature Deformation and Burst Characteristics of Recrystallized Zircaloy 4 Tubing, WAPD-TM-900 (January 1970).

IV-11. R. H. Geiss and W. F. Marley, Jr., Survey of Research on Superplasticity Worldwide, Army Foreign Srience and Technology Center, Charlottesville, Va. (December 10, 1973).

IV-12. D. R. Dreger, "Metals that Form Like Plastics", Machine Design (August 8, 1974).

IV-13. D. R. Dreger, "Carbon Steels Join the Superplastic Metals", Machine Design (April 3, 1975).

IV-14. J. E. Harbottle, The Temperature and Neutron Dose Dependence of Irradiation Growth in Zircaloy-2, Irradiation Effect on Structural Alloys for Nuclear Reactor Applications, ASTM-STP-485'(1970) pp 287-299.

IV-15. R. C. Daniel, "In-Pile Dimensional Changes of Zircaloy-4 Tubing Having Low Hoop Stresses (Light Water Breeder Reactor Development Program)", Nuclear Tech., 14 (May 1972) pp 171-186.

IV-16. W. R. Smally, Evaluation of Saxton Core III Fuel Materials Performance, WCAP-3385-57 (1974).

IV-17. J. B. Melehan, Yankee Core Evaluation Program Quarterly Progress Report for the Period Ending September 30, 1969, WCAP-3017-6091 (December 1969).

IV-18. E. T. Laats and P. E. MacDonald, NRC-OECD Halden Project Fuel Behavior Test Program - Experimental Data Report for Test Assemblies IFA-226 and IFA-239, ANCR-1270 (December 1975).

IV-19. P. H. Kreyns, quoted by E. Duncombe, J. E. Mayer, W. A. Coffman in Comparisons with Experiment of Calculated Changes and Failure Analysis of Irradiated Bulk Oxide Fuel Test Rods Using the CYGRO-1 Comnuter Program, WAPD-TM-583 (September 1966). 
IV-20. R. Daniel, In-Pile Dimensional Changes of Zircaloy-4 Tubing Having Low Hoop Stresies (I.WBR. Development Program), WAPD-TM-973 (July 1971).

IV-21. V. Fideris, "The Effect of Cold-Work and Stress-Relieving on the Irradiation Growth Behavior of Zirconium Alloys", J. Nucl. Mat., 46 (1973) pp 356-360.

IV-22. R. V. Hesketh, "Non-linear Growth in Zircaloy-4", J. Nucl. Mat., 30 (1969) pp 217-222.

IV-23. V. Fidleris, Summary of Experimental Results of In-Reactor Creep and Irradiation Growth of Zirconium Alloys, AECL 4766 (to be published).

IV-24. P. J. Berensun, Filrr-Builing Heul Trunsfer from a Ilorizontal Surface, Vol. 83 (August 1961) pp 351-358.

IV-25. D. C. Groeneveld, An Investioation of Heat Transfer in the Liquid Deficient Régimes, AECL-3281 Rev. 1 (December 1968), Rev. 2 (August 1969).

IV-26. R. S. Dougall and W. M. Rohsenow, Film-Boiling on the Inside of Vertical Tubes with Upward Flow of the Fluid at Low Qualities, MIT-TR-9079-26 (1963). 
DISTRIBUTTION RECORD FOR ANER-1296

\section{External}

245 - NRC-1, Basic Distribution

13 - NRC-2, Water Reactor Safety Research Systems Engineering

40 - NRC-3, Water Reactor Safety Research Fue1 Behavior

52 - NRC-4, Water Reactor Safety Research Analysis Development

2 - H. J. C. Kouts, NRC

\section{Interna?}

1 - Chicago Patent Group - ERDA 9800 South Cass Avenue Argonne, Tlitinois $\quad 60439$

3 - A. T. Morphew, Classification and Technical Information Officer ERDA-ID Idaho Fa'Ts, Idaho 83401

1 -R. J. Beers, IDD

T P. E, Littencker, ID

$T$. = R. E. Swanson, ID

1 - V. A. Walker, ID

1 - R. E. Wood, IB

37 - INEL Technical Library

20 - Author 